\title{
Control of Pests and Diseases
}

\author{
Alan J. Terry
}

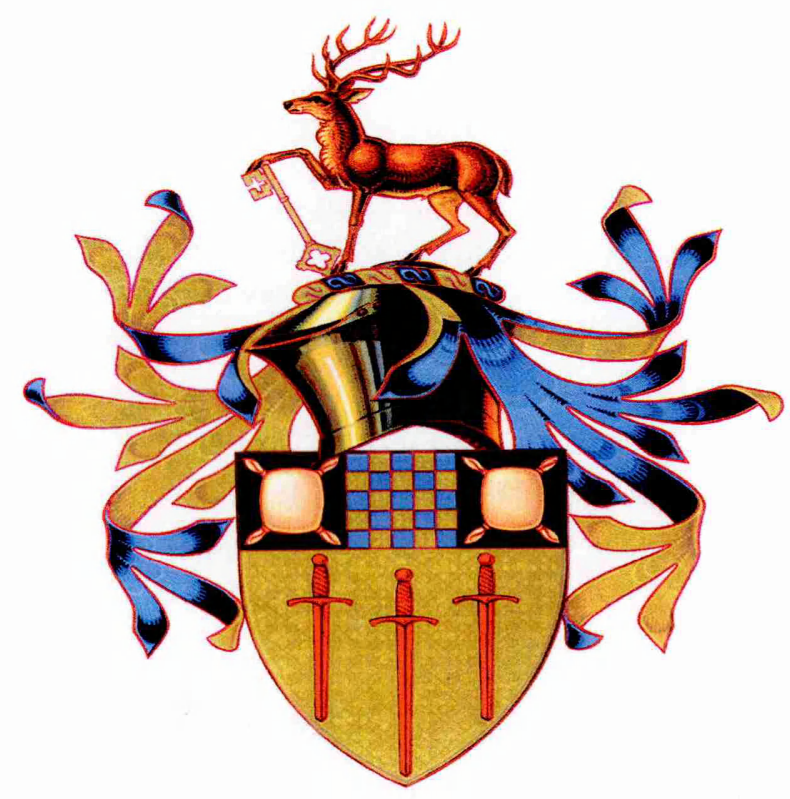

A thesis submitted for the degree of

Doctor of Philosophy

Department of Mathematics

Faculty of Engineering and Physical Sciences

University of Surrey

Guildford, Surrey

February 2009 
ProQuest Number: 27731950

All rights reserved

INFORMATION TO ALL USERS

The quality of this reproduction is dependent upon the quality of the copy submitted.

In the unlikely event that the author did not send a complete manuscript and there are missing pages, these will be noted. Also, if material had to be removed, a note will indicate the deletion.

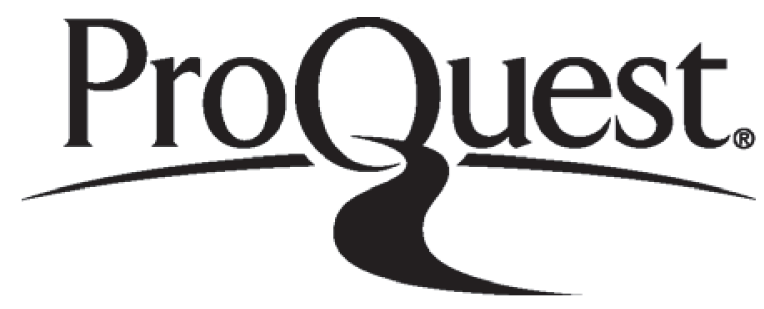

ProQuest 27731950

Published by ProQuest LLC (2019). Copyright of the Dissertation is held by the Author.

All rights reserved.

This work is protected against unauthorized copying under Title 17, United States Code Microform Edition (C) ProQuest LLC.

ProQuest LLC.

789 East Eisenhower Parkway

P.O. Box 1346

Ann Arbor, Ml $48106-1346$ 


\begin{abstract}
This is a doctoral thesis in the control of pests and diseases. The type of pest of concern to us will lead a life cycle with distinct developmental stages, specifically juvenile and adult. We will construct adult-stage impulsive culling regimes to eradicate a population of such a pest as well as discover that poorly planned regimes may, perversely, benefit the pest. Real-world applications will include the control of insects that act as crop pests or that act as vectors in the spread of human or livestock diseases. Our disease control work will focus on three problems, essentially unrelated except in so far as each has been little explored until now. In the first of these problems, we investigate an SIR model with growing total population and a contact rate that grows with the population; in the second we seek successful pulse vaccination strategies in a metapopulation SIR model; and in the third we derive and study an SIR model with a gestation delay. Suggestions for future research are given at the end.
\end{abstract}

\title{
Acknowledgements
}

First and foremost I would like to thank my supervisor, Dr. Stephen Gourley, for helping to find me suitable problems for a doctoral thesis. I also thank him for his guidance in solving these problems and his careful proof-reading of my work. Finally I express gratitude to the Engineering and Physical Sciences Research Council for funding this research. 


\section{Contents}

1 General introduction $\quad 1$

I Pest control 3

2 Introduction to pest control $\mathbf{5}$

2.1 Insect life cycles and stage-specific culling . . . . . . . . . 5

2.2 Pest control problems in this thesis $\ldots \ldots \ldots \ldots \ldots \ldots$

2.3 Caution with pesticides $\ldots \ldots \ldots \ldots$

3 Culling on one patch $\quad 11$

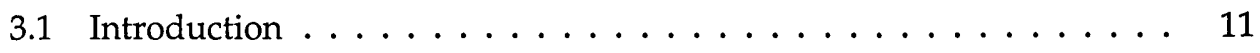

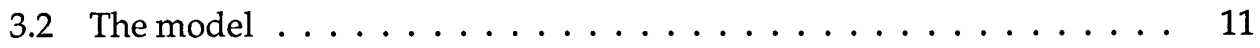

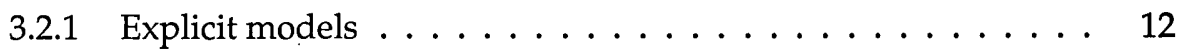

3.3 Extinction and endemicity . . . . . . . . . . . . 15

3.3.1 Linear model . . . . . . . . . . . . . . . . . . 15

3.3.2 Nicholson model . . . . . . . . . . . . . . . . . . . . . 19

3.3 .3 Allee model . . . . . . . . . . . . . . . . 21

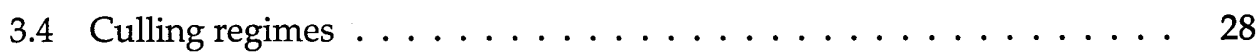

3.4 .1 Linear model . . . . . . . . . . . . . . . . . . . . 29

3.4 .2 Nicholson model . . . . . . . . . . . . . . . . . 34

3.4 .3 Allee model . . . . . . . . . . . . . . 35

3.4 .4 Regime existence $\ldots \ldots \ldots \ldots \ldots \ldots \ldots$

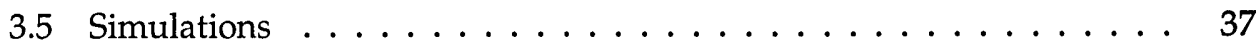

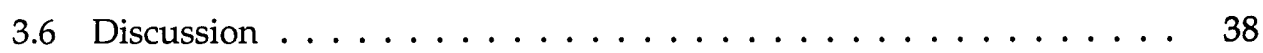

4 Infrequent culling $\quad 41$

4.1 Introduction $\ldots \ldots \ldots \ldots \ldots \ldots \ldots \ldots \ldots \ldots \ldots$

4.2 The model . . . . . . . . . . . . . . . . . . . . . 42

4.3 The role of the birth function $\ldots \ldots \ldots \ldots \ldots$

4.4 A simple birth function . . . . . . . . . . . . . . 44 
4.5 Model analysis . . . . . . . . . . . . . . . . . . . . . . . 45

4.6 Influence of culling on long-term mean population . . . . . . . . . 48

4.7 Simulations . . . . . . . . . . . . . . . . 51

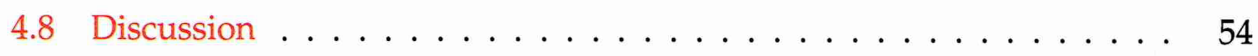

5 A two-patch model $\quad 56$

5.1 Introduction . . . . . . . . . . . . . . 56

5.2 The model . . . . . . . . . . . . . . . . . . 57

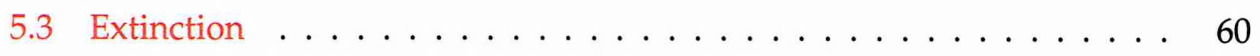

5.4 Minimum viable population for Allee births . . . . . . . . . 62

5.5 Population boundedness when birth functions are bounded . . . . 65

5.6 Endemicity . . . . . . . . . . . . . . . . . 66

5.6 .1 Linear births . . . . . . . . . . . . . . . 66

5.6 .2 Nicholson or Allee births . . . . . . . . . . . . . 68

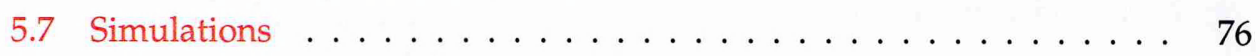

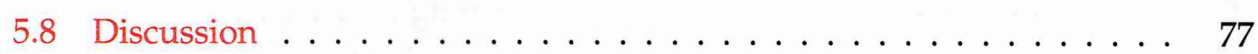

6 Culling on two patches $\quad \mathbf{8 0}$

6.1 Introduction . . . . . . . . . . . . . . . . 80

6.2 Culling on one patch . . . . . . . . . . . . . 81

6.3 Culling regimes . . . . . . . . . . . . . . . . 85

6.4 Definition of our culling regimes $\ldots \ldots \ldots \ldots$. . . . . . . 92

6.5 A proof that our regimes are successful . . . . . . . . . . . 93

6.6 Analysis of the regimes . . . . . . . . . . . . . . . . . . . . 105

6.7 Eradication in finitely many culls . . . . . . . . . . . . . 106

6.8 Simulations . . . . . . . . . . . . . . . . . . . . . . . 108

6.9 Discussion . . . . . . . . . . . . . . . . . . . 113

$\begin{array}{ll}\text { II Disease control } & 115\end{array}$

$\begin{array}{lll}7 & \text { Introduction to disease control } & \mathbf{1 1 7}\end{array}$

7.1 Mathematical epidemiology . . . . . . . . . . . . . . . . 117

7.2 Types of model . . . . . . . . . . . . . . . . . . . . . . 118

7.3 Frequent assumptions . . . . . . . . . . . . . . . . . . . 119

7.4 Vaccination strategies . . . . . . . . . . . . . . . . . . . 120

7.5 Disease control problems in this thesis . . . . . . . . . . . 122

7.6 Hindrances to disease control . . . . . . . . . . . . . . . 122 
8 Population-dependent contact rates in an SIR model $\quad 125$

8.1 Introduction . . . . . . . . . . . . . . . . 125

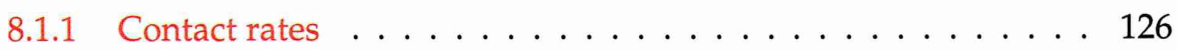

8.2 An SIR model . . . . . . . . . . . . . . . . . . . 128

8.3 Susceptible population . . . . . . . . . . . . . . . . . . 130

8.4 Vaccination strategies . . . . . . . . . . . . . . . . . . 137

8.5 Simulations .............................. 141

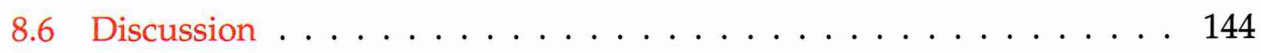

9 PVS in a metapopulation SIR model $\quad 146$

9.1 Introduction . . . . . . . . . . . . . . . . . . . 146

9.2 The model . . . . . . . . . . . . . . . . . . . . 147

9.3 Natural extinction and endemicity . . . . . . . . . . . . . . . . . . 149

9.4 Pulse vaccination strategies . . . . . . . . . . . . . . . . . 150

9.5 Regime existence . . . . . . . . . . . . . . . . . . . . 160

9.6 Simulations . . . . . . . . . . . . . . . . 160

9.7 Discussion . . . . . . . . . . . . . . . . . 164

10 Epidemiological models with a gestation delay 166

10.1 Introduction . . . . . . . . . . . . . . . . . . . 166

10.2 An SIR model . . . . . . . . . . . . . . . . . . . . 167

10.3 A simple model . . . . . . . . . . . . . . . . . . . . . 171

10.4 Model analysis . . . . . . . . . . . . . . . . . . . . . 172

10.5 Vaccination strategies . . . . . . . . . . . . . . . . . 178

10.6 Simulations . . . . . . . . . . . . . . . . . . 184

10.7 Insect pathogen dynamics . . . . . . . . . . . . . . . . . . 184

10.7 .1 Two models . . . . . . . . . . . . . . . . . . . 185

10.7 .2 Model analyses . . . . . . . . . . . . . . . . . . . 187

10.8 Discussion . . . . . . . . . . . . . . . . . . 192

$\begin{array}{ll}\text { III Concluding remarks } & 193\end{array}$

11 Concluding remarks $\quad 195$

11.1 Pest control summary . . . . . . . . . . . . . . . . . . 195

11.2 Pest control extensions . . . . . . . . . . . . . . . . . . 196

11.3 Disease control summary . . . . . . . . . . . . . . . . . . . 197

11.4 Disease control extensions . . . . . . . . . . . . . . . . . 198 
$\begin{array}{ll}\text { Bibliography } & 201\end{array}$

$\begin{array}{ll}\text { Index } & 208\end{array}$ 


\section{Chapter 1}

\section{General introduction}

"Who controls the past controls the future.

Who controls the present controls the past."

George Orwell (1903 - 1950)

The purpose of this doctoral thesis is to extend our understanding of certain aspects of pest control and disease control. Various mathematical models will be analysed, appropriate control regimes constructed, and comments will be made on the implications of our results in terms of which real-world decisions to take. All of the material in the thesis is broadly related by the potential to make life easier for people. The material is organised into three parts: pest control, disease control, and concluding remarks.

In our pest control work, we will essentially be concerned with tropical insect species. The type of insect of interest to us will lead a life cycle consisting of distinct developmental stages which culminate in an adult stage capable of reproducing. The form of control to be investigated will be adult impulsive culling because this is a common real-world control method. Practical applications will include the control, by the regular spraying of pesticides, of agricultural pests which eat crops or of creatures, such as mosquitoes, which act as a vector in the spread of infectious diseases in human or livestock populations. Serious diseases spread by mosquitoes include malaria (which kills over a million people every year), yellow fever, West Nile virus, dengue fever, and various forms of encephalitis [24]. Notice, then, that pest control is an area of disease control if the pest we are seeking to control is a vector in the spread of disease.

The main achievements of our pest control work will be the construction of successful control regimes for a creature that either inhabits a single region or inhabits two regions between which individuals may migrate. The regions could represent farms, for example. We shall discover as well, by a plausible mathematical model, that infrequent applications of pesticide can, perversely, increase the average population of a pest. Thus we will gain an understanding both of when adult impulsive culling eradicates a pest and of when it benefits a pest. An introduction to pest control is included at the start of part I. 
In the second part of the thesis we examine three epidemiological models. There is no common theme to these models besides the exploration of some aspect of disease control hitherto unexplored but of practical significance. In the first model we investigate pulse and constant vaccination strategies in a model in which the total population is growing and the contact rate grows with the population. A pulse vaccination strategy is found to be ineffective in the long run but a constant vaccination strategy is found to bring the disease under some control.

In our second epidemiological model, a disease circulates in a population inhabiting $n$ different regions between which migration may occur. The different regions could be cities, counties, or countries linked by common borders or direct flight routes. We ask if a disease endemic in at least some of the regions can be eradicated in all regions simultaneously by the implementation of an independent pulse vaccination strategy in each region. We conclude that it can.

Few epidemiological models for mammalian species in the literature have included a gestation delay, so in our final model we incorporate such a delay. Successful pulse and constant vaccination strategies are constructed for the model. We also discuss the related field of insect pathogen dynamics. A separate introduction to disease control is included at the start of part II.

In the third part of the thesis we summarise our achievements and place them in a wider context. Suggestions for future research are given.

The mathematical models of this thesis contain either delay differential equations or ordinary differential equations. Many of the calculations involve impulses in such equations. Impulses in delay differential equations are still to receive major attention in the literature but the curious reader may find information on pages 90-95 of [79] and in [80]. A good introduction to delay differential equations may be found in [95]. However, it is not necessary for the reader to be familiar with delay differential equations in order to understand this thesis. Our methods are elementary and new concepts are explained as and when they are needed. The only real prerequisite is a basic grasp of calculus and of ordinary differential equations. 


\section{Part I}

Pest control 
MATERIAL REDACTED AT REQUEST OF UNIVERSITY 


\section{Chapter 2}

\section{Introduction to pest control}

"There are scores of thousands of human insects who are ready at a moment's notice to reveal the will of God on every possible subject."

George Bernard Shaw (1856 - 1950)

\subsection{Insect life cycles and stage-specific culling}

Various species feed on crops or act as a vector in the spread of human or livestock diseases. This frequently leads people to implement methods of pest control. The method of control will depend on the type of pest, for example, or the level of resources available for its control, or the severity of the problem. The environmental consequences of the control method also matter.

We restrict our attention to pests that lead a stage-structured life cycle. By this we mean that the life cycle of the pest can be split into distinct stages. We have in mind insects, which often pass through quite different phases before reaching a final adult phase capable of reproducing, sometimes in staggering quantities.

Insect life cycles can be complicated, and they vary significantly within the class. But there are large taxonomic groups or clades with life cycles fitting the same basic pattern. More than four fifths of the known insect species (there are well over a million known species) belong to the superorder Endopterygota (see [17] and p. 13 in [117]). The Endopterygota (literally "internal wings formed") develop wings inside the body and are also called the Holometabola ("whole change") because they undergo an elaborate metamorphosis. The life cycle contains four stages, namely embryo, larva, pupa, and imago (or adult) [17]. For instance, in the life cycle of a moth, the embryo grows within the egg, hatching into the larval stage caterpillar, before entering the pupal stage within its cocoon and finally emerging as an adult moth imago (see figure 2.1). The Endopterygota consist of numerous orders, some of which are extinct. Extant orders include coleoptera (beetles), diptera (true flies), and lepidoptera (butterflies and moths). There are literally thousands of pest species contained in these last three orders, so we shall not name them all. An example pest from the coleoptera is the colorado potato beetle [16]. The diptera include mosquitoes (which 
MATERIAL REDACTED AT REQUEST OF UNIVERSITY

spread diseases such as malaria, yellow fever, dengue fever, and West Nile virus) [24] and fruit flies [13]. The lepidoptera include the codling moth (see figure 2.1), the caterpillar of which is an apple pest [26].

Insect life cycles are critically influenced by climate. In temperate zones, breeding is typically restricted to the summer months whereas tropical regions may admit year-round reproduction. The pest control models of this thesis will be based on an assumption of year-round breeding. Since we do not wish to give an unbalanced depiction of insects, we note in passing that they probably help people more than they hinder them - without insect pollination, many crops would fail.

Many species in the Endopterygota experience short embryonic and pupal stages relative to the larval and adult stages during their summer breeding seasons or yearround in tropical areas. When this happens we may rudely approximate the life cycle by two stages, the larval and adult stages. Alternatively we may draw a distinction between juvenile insects as those not yet capable of reproducing and adults as those that are capable. In this sense as well we may view the pest as having a life cycle with two stages. The reason we mention this is that our later mathematical models make a simplifying assumption that the pest life cycle may be approximated by two stages, 
each with a simple death function.

For a pest with a stage-structured life cycle, a particular stage can be targeted for control. For instance, the adult stage of an insect pest can be targeted with an appropriate pesticide (a pesticide intended to kill adults is called an adulticide). Adult insects can be easier to find, and therefore kill, because they are frequently driven into the open by the instinct to search for a mate. If the adult population is brought under sufficient control, then reproduction and hence the entire pest population are brought under control. Thus, even if it is only the larvae of a species that cause harm, the impact of the pest can be managed by controlling adult numbers.

Agricultural pesticides are often applied by aerial spraying at regular intervals (once a week, say). This may be achieved by a light aircraft known as a crop duster or by other methods. Each application of pesticide can be deemed a cull, since the word "cull" means a brief but controlled extermination episode. In terms of applying pesticides, the culls can be so brief in relation to the inter-cull period (the time elapsing between consecutive culls) as to sensibly admit the rather elegant modelling assumption that they are in fact instantaneous. The idea that culls are impulsive can yield simpler mathematics.

\subsection{Pest control problems in this thesis}

The term patch is often used by mathematicians to mean "region". This convention will be followed by us.

In chapter 3, we examine three models for a pest inhabiting a single patch. The models differ from each other by the choice of birth function. The birth functions that we consider will be labelled linear, Nicholson, and Allee, and they have all previously appeared in the literature. Since it is unnecessary to apply pesticides if a pest is crashing naturally to extinction, we first establish conditions, for each model, such that the pest naturally goes extinct or is naturally endemic. Then we construct adult impulsive culling regimes that eradicate the pest, which could be implemented if the pest were endemic and causing serious harm. Our results will be corroborated by simulations. A simulation will also suggest that culling infrequently may increase the average population of a pest.

In chapter 4 we seek analytical confirmation that culling infrequently can, perversely, benefit a pest population. This will be achieved by considering a model on a single patch in which the birth function contains a step.

In chapter 5 we describe a model for a creature inhabiting two patches between which migration may occur. The patches could represent neighbouring farms, for example. The birth function on either patch may be of linear, Nicholson, or Allee type, giving rise to nine different cases. We find conditions in all cases such that the pest naturally goes extinct on both patches, and conditions in some cases such that it is naturally endemic.

Finally, in chapter 6, we impose on the model of chapter 5 an adult impulsive culling regime on each patch. We find conditions on the regimes such that the pest will be eradicated on both patches simultaneously. The regime on one patch will be essentially independent from the regime on the other patch to reflect the possibility that the patches represent farms with different owners where each owner has autonomy in their pest control decisions. 


\subsection{Caution with pesticides}

Applying pesticides when they are not necessary is potentially unwise. In a thesis containing results promoting pesticide use, we recognise our responsibility to urge caution. Insects adapt quickly to pesticides, enabling them to build up a resistance [64]. So using pesticides when they are not necessary will only make them less effective when they really are needed.

Often a pest insect population will have natural controlling factors such as predators or parasites. But an insecticide application can kill both pest and its controlling agents. The disruption to the natural controlling factors may be even greater than it is to the pest, enabling the pest population to subsequently rebound to a level higher than before the insecticide was used. This phenomenon is called pest rebound or pest resurgence [64]. Loss of predator species can also lead to a related phenomenon called secondary pest outbreaks, an increase in problems from species which were not originally very damaging due to loss of their predators or parasites [64]. An estimated third of the 300 most damaging insects in the USA were originally secondary pests and only became a major problem after the use of pesticides [109].

The environmental impact of pesticides has been well documented. The United States Department of Agriculture (USDA) and United States Fish and Wildlife Services (USFWS) estimate that over 67 million birds and between 6 and 14 million fish are killed by pesticides each year in the USA [109]. Also the breakdown products of pesticides can be even more dangerous to some animals than the pesticide itself, or can be dangerous even when the pesticide itself is not thought to pose a threat. Scientists in California warn that breakdown products of certain common pesticides could be 10 to 100 times more toxic to amphibians than the original pesticide [9]. Application of pesticides to crops that are in bloom can kill honeybees, which act as pollinators. The USDA and USFWS estimate that US farmers lose at least $\$ 200$ million a year from reduced crop pollination because pesticides applied to fields eliminate about a fifth of honeybee colonies in the US and harm an additional 15\% [109]. Finally the World Health Organisation and the UN Environment Programme estimate that each year, 3 million workers in agriculture in the developing world experience severe poisoning from pesticides, about 18,000 of whom die [109].

Given all of these concerns, it may well be asked why we use pesticides at all? There are in fact numerous reasons but most important are the immense pressures faced by commercial farmers. Firstly, there is the demand to feed and clothe a global population that has been growing steadily for centuries [34]. One study found that not using pesticides reduced crop yield by about $10 \%$ [96] but, without any form of control, some pests have the ability to devastate entire industries - the boll weevil, for instance, which ruined the United States cotton industry in the 1920s and which remains the most destructive cotton pest in North America [14]. Secondly, farmers possess the desire to make a profit. In the USA, farmers get an estimated four-fold return on money they spend on pesticides [92]. Pesticides are used mostly in agriculture - for example, $70 \%$ of pesticides sold in the USA are used in agriculture - but they also play a role in disease control. Scientists have estimated that DDT and related pesticides have saved over 7 million human lives since 1945 by preventing the transmission of diseases such as malaria, bubonic plague, sleeping sickness, and typhus [109]. Pesticides have various other uses too. Herbicides kill weeds in gardens, fly spray kills the irritating house fly or troublesome wasps, insecticides kill termites which can damage structures such as houses, insecticides can also protect animals from illnesses that can be caused by parasites such as fleas, rodenticides in food stor- 
age facilities manage rodent populations which may eat the food or spread disease, and so on. The importance of pest control is clear and pesticides currently play a huge role with very strong benefits.

With the large-scale introduction of synthetic pesticides like DDT in the 1940s, a wave of optimism encouraged the belief that we could soon eradicate all pests if we so desired. But the idea of eradicating a species has become less acceptable firstly, because it could disrupt an ecosystem in an unpredictable way, causing other pests to become more problematic; secondly, because, with many pests, eradication is virtually impossible anyway; and thirdly, because causing deliberate extinction is less acceptable ethically than it used to be. The emphasis has shifted towards sustainable control methods. The drawbacks associated with pesticides are sufficiently serious that it is difficult to envisage their large-scale use as permanently sustainable. We cannot pump millions of tons of industrial pesticides into the environment every year [108] with impunity. Other forms of pest control also currently play a role (see table 2.1), and perhaps they will take on greater significance in due course. Whatever the long-term future is for pesticides, they will remain in large-scale use for many years. For as long as they do, we urge that they only ever be applied with caution. 


\begin{tabular}{|c|c|}
\hline Method & Comments \\
\hline Biocontrol & $\begin{array}{l}\text { Also called biological control, this is the introduction of predators, parasites, or } \\
\text { pathogens to target a pest }[7,97] \text {. It is sensible to research the impact an intro- } \\
\text { duced species will have on an ecosystem before introducing it. Effective biocontrol } \\
\text { agents include predatory fish that feed on mosquito larvae, such as mosquitofish, } \\
\text { ciprinnids (carps and minnows) [10], and killifish. An example of a poorly planned } \\
\text { introduction with disastrous consequences is the cane toad in Australia [22]. }\end{array}$ \\
\hline Crop rotation & $\begin{array}{l}\text { Popular in the tropics. The type of crop sewn in a particular field is changed from } \\
\text { one year to the next, which prevents those pests and plant diseases dependent on } \\
\text { a specific crop from building up to unmanageable levels [89]. After three or four } \\
\text { different crops, the original crop may be re-planted and the cycle or rotation begins } \\
\text { anew. Crops may also be rotated around the different fields of a farm. Rotating } \\
\text { crops helps to maintain soil fertility. }\end{array}$ \\
\hline Slash/mulch & $\begin{array}{l}\text { Begins with a "green manure" - plants are grown to improve the soil, suppress } \\
\text { weeds, limit erosion, and increase the nitrogen content of the soil. The most com- } \\
\text { mon green manure used in the tropics is the Velvet bean which produces a blan- } \\
\text { ket of vines. Once the blanket is several centimetres thick, it is chopped down } \\
\text { with a machete ("slashed"), and the vines are chopped up. This produces a dense } \\
\text { "mulch" on top of the ground that both inhibits weeds and adds vital nutrients to } \\
\text { the soil. Corn or other crops are then planted directly into this mulch [1]. }\end{array}$ \\
\hline GM crops & $\begin{array}{l}\text { In recent years it has become possible to alter the DNA of crops through genetic } \\
\text { engineering, enabling crops to be endowed with new properties that help them } \\
\text { to grow better. Traits that have been engineered into maize, for example, include } \\
\text { resistance to herbicides and the incorporation of a gene that codes for the Bacillus } \\
\text { thuringiensis (Bt) toxin which protects the plants from insect pests [57]. }\end{array}$ \\
\hline Slash and burn & $\begin{array}{l}\text { This involves the cutting and burning of the natural vegetation in a forested area } \\
\text { and then farming the cleared patch for a few years until the soil loses its fertility, } \\
\text { whereupon farmers move on and leave the area to regrow [63]. Burning the vegeta- } \\
\text { tion not only removes it but may also release a pulse of nutrients. Fire temporarily } \\
\text { drives off soil micro-organisms and pests as well. Slash and burn is an ancient } \\
\text { practice but becomes unsustainable in a region with high population density. }\end{array}$ \\
\hline IPM & $\begin{array}{l}\text { Integrated Pest Management (IPM) is a relatively new and environmentally sen- } \\
\text { sitive approach to pest control which uses ecological control methods, with pesti- } \\
\text { cides as a last resort [21]. IPM attempts to recognise the more widespread impacts } \\
\text { of an action on an ecosystem, so that natural balances are not upset. Typically, } \\
\text { an IPM program will begin with a survey of the pest in question. Only then are } \\
\text { the best and most effective methods of control determined and utilised, with an } \\
\text { emphasis on control rather than eradication. }\end{array}$ \\
\hline
\end{tabular}

Table 2.1: Methods of pest control that do not usually involve pesticides. 


\section{Chapter 3}

\section{Impulsive adult culling of a tropical pest with a stage-structured life cycle}

"The butterfly counts not months but moments, and has time enough."

Rabindranath Tagore (1861 - 1941)

\subsection{Introduction}

We have reviewed insect life cycles and the concept of adult impulsive culling for a pest with a two-stage structured life cycle in our introduction to pest control (chapter 2). In this chapter, we begin (section 3.2) by describing a model for the adult population of a pest with a two-stage-structured life cycle, a general birth function, and which breeds year-round. In subsection 3.2.1 we consider three different types of birth function that have appeared in the literature. In section 3.3 we show, for each of these birth functions, that the adult population may naturally go extinct or persist indefinitely subject to conditions on model parameters and initial data. In section 3.4 we construct conditions on an impulsive adult culling regime that guarantee eradication of the adult population. Simulations are included in section 3.5 and a discussion in section 3.6 ends the chapter. Note that the material in this chapter has been accepted for publication in Nonlinear Analysis: Real World Applications; see reference [133].

\subsection{The model}

Suppose we have a population of a pest with a life cycle that can be modelled by two stages, namely a juvenile or immature stage and an adult or mature stage. Only the adult stage can reproduce. (This last assumption is not unnecessary - some types 
of insect, such as aphids (which are not in the Endopterygota), can be born pregnant! [68].) Assume births and natural deaths may occur continuously in time. Also assume an individual becomes mature on reaching age $\tau$ where $\tau$ is a positive constant called the maturation age, provided the individual lives that long. Let the per capita death rate of juveniles be a constant $\mu_{j}>0$. Denote by $N(t)$ the total number of adults at time $t$ and suppose we have initial data for the adult population expressed as an initial function:

$$
N(t)=\phi(t) \geq 0 \text { for } t \in[-\tau, 0] .
$$

In many insect species, the adults will die whilst still of a reproducing age. Therefore, let the birth rate be a function of the entire adult population, specifically $b(N(t))$. Also let the adult death rate be a function of the adult population, namely $d(N(t))$.

Given all of these assumptions, it is possible to derive a delay differential equation governing the evolution of the adult population. Such a derivation has been performed by Simons and Gourley [125], who find that:

$$
\frac{d N(t)}{d t}=e^{-\mu_{j} \tau} b(N(t-\tau))-d(N(t)) \text { for } t \geq 0 .
$$

By saying the derivative $\frac{d N(t)}{d t}$ exists for $t \geq 0$, it should be understood that the left derivative exists at $t=0$ and that the derivative exists for $t>0$. A solution to (3.2), subject to (3.1), will exist and be unique if the functions $b, d$, and $\phi$ are suitably sensible. A proper discussion of the solutions to delay differential equations may be found in Kuang's book [95]. We will study three specific cases for (3.2) which, for continuous non-negative initial data $\phi$, will have unique solutions for $t>0$.

The terms in (3.2) may be interpreted ecologically. The net rate of change at time $t$ of the adult population, $\frac{d N(t)}{d t}$, is the rate of new entries minus the rate of departure. But the rate of new entries into the adult population at time $t$ is the number of immatures becoming mature at time $t$ (which is $b(N(t-\tau))$ ) scaled by the proportion that have survived to maturity (which is $e^{-\mu_{j} \tau}$ ), and the rate of departure of individuals from the adult population at time $t$ is simply the adult death rate $d(N(t))$.

Assume that the birth and death functions satisfy the following biologically sensible requirements:

$$
\begin{array}{lll}
b(0)=0 & \text { and } & b(x)>0 \text { for } x>0 \\
d(0)=0 & \text { and } & d(x)>0 \text { for } x>0 .
\end{array}
$$

Observe that the model (3.2), subject to (3.1), (3.3), and (3.4), can be shown to possess the property of positivity, that is, $N(t) \geq 0$ for $t \geq 0$ (proposition 2.1, [125]). The special case $N(t)>0$ for $t \geq 0$ will be referred to as strict positivity.

\subsubsection{Explicit models}

It is common practice in population models to let the per capita death rate be a constant $[60,82,123,129]$. Henceforth assume the adult per capita death rate is a constant $\mu>0$. Then $d(N(t))=\mu N(t)$. Sometimes we shall write $N$ as a shorthand for $N(t)$. We shall explore three different birth functions:

1) $b(N)=\lambda N$ where $\lambda$ is a positive constant (linear birth function) 
2) $b(N)=\lambda_{1} N e^{-\lambda_{2} N}$ where $\lambda_{1}, \lambda_{2}$ are positive constants (Nicholson birth function)

3) $b(N)=\alpha_{1} N^{2} e^{-\alpha_{2} N}$ where $\alpha_{1}, \alpha_{2}$ are positive constants (Allee birth function)

These have all appeared in the literature although, as far as we are aware, the latter two functions have not been given explicit names. The linear birth function results from the straightforward assumption that the birth rate per adult is a constant $\lambda>0$. Hence if there are more adults, then they produce more offspring. We have named the Nicholson birth function after A. J. Nicholson, whose laboratory work on the Australian blowfly Lucillia cuprina $[115,116]$ motivated Gurney et al [82] to derive a population model with a birth function of the form $\lambda_{1} N e^{-\lambda_{2} N}$ for $\lambda_{1}, \lambda_{2}$ positive constants. This function increases linearly for small $N$ but, as $N$ increases, it reaches a maximum and then decreases to zero. These facts capture the idea that a small population can breed quickly until it grows enough that factors such as overcrowding or competition hinder mating.

We have given the Allee birth function its name because it may be associated with a phenomenon called the Allee effect (see [45] and p. 23 in [55]) in which a population can be low enough as to be unsustainable, making extinction inevitable. We will draw attention to this association in a moment. The Allee birth function is small for large $N$, just as the Nicholson birth function is, but it is also small for small $N$, reflecting the idea that reproduction in a small population may be difficult since it may be harder for individuals to find a mate and there will be little group defence.

The three explicit models we will consider may be written:

$$
\begin{aligned}
& \frac{d N(t)}{d t}=\lambda N(t-\tau)-\mu N(t) \text { for } t \geq 0 \quad \text { (linear model) } \\
& \frac{d N(t)}{d t}=\lambda_{1} N(t-\tau) e^{-\lambda_{2} N(t-\tau)}-\mu N(t) \text { for } t \geq 0 \quad \text { (Nicholson model) } \\
& \frac{d N(t)}{d t}=\alpha_{1}(N(t-\tau))^{2} e^{-\alpha_{2} N(t-\tau)}-\mu N(t) \text { for } t \geq 0 \quad \text { (Allee model) }
\end{aligned}
$$

Notice that we have, for ease of notation, absorbed $e^{-\mu_{j} \tau}$ in (3.2) into constants in the birth functions. These models remain subject to the initial data (3.1).

The three different birth functions are plotted for example parameter values in figure 3.1, along with example death functions. If we set $\tau=0$ in (3.1) and in the linear, Nicholson, or Allee model equations, then the global behaviour of $N(t)$ is easily deduced by standard phase portrait techniques. In particular it is clear that an Allee effect will hold for the Allee model (there is always a minimum viable population). Allee effects have been observed in various kinds of creature, including insects [134, 139]. Many of the results that hold when $\tau=0$ can be shown to carry over into the case $\tau>0$, as we shall see in the next section.

If the pest population grows quickly, then the implementation of a control method becomes important. If the population will crash naturally to extinction, it is unnecessary and even unwise to impose a pest control stratagem. After all, why apply pesticides if they are not needed? Such action would waste resources and time and could promote pesticide resistance in other pest species. As a rule, pesticide use should be minimised to limit the environmental degradation that may be caused. It is therefore sensible to understand what the pest population is likely to do before deciding how best to act. Hence in the next section we explore the range of natural behaviours exhibited by our three models. 

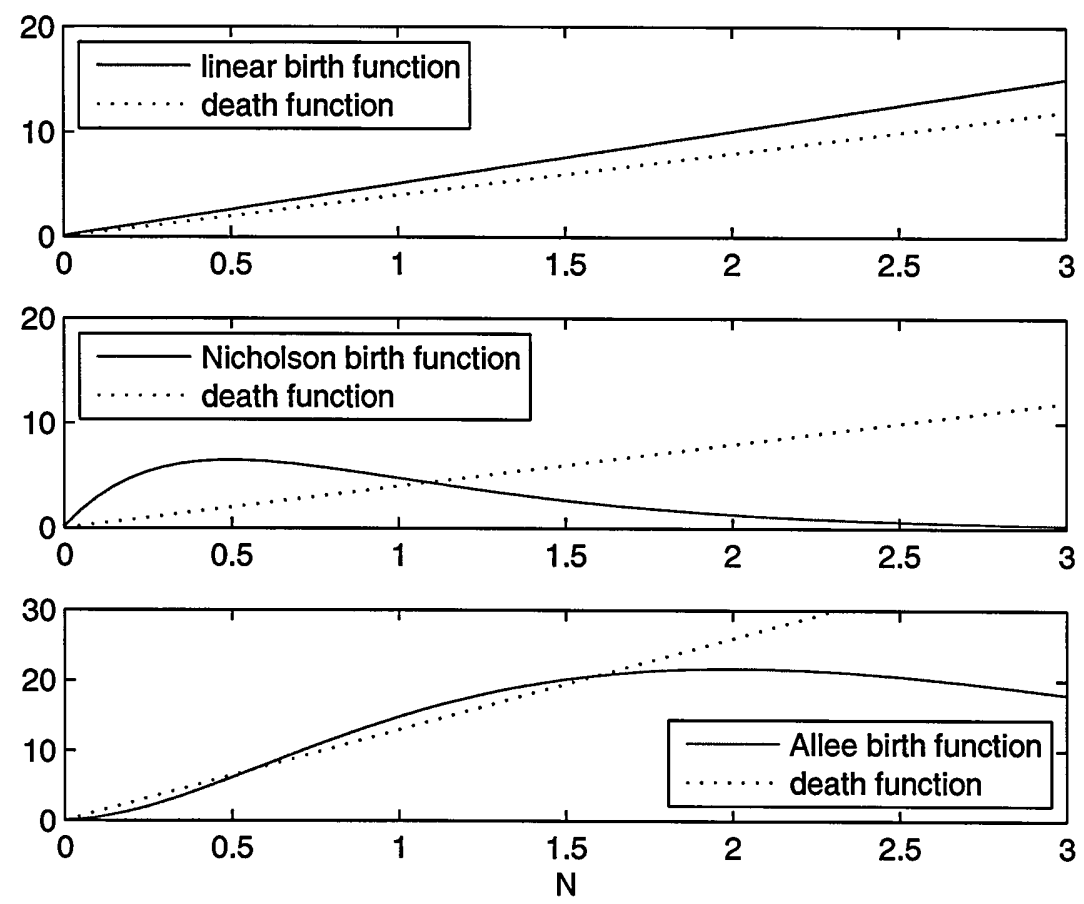

Figure 3.1: Example birth and death functions. Parameter choices here are: $\lambda=5$, $\lambda_{1}=35, \lambda_{2}=2, \alpha_{1}=40, \alpha_{2}=1, \mu=4$ in the top two plots and $\mu=13$ in the bottom plot. (All of the simulations in this thesis were performed and created in matlab.) 


\subsection{Extinction and endemicity}

By endemicity we mean persistence, or the opposite of extinction. Thus if the pest remains endemic, it must persist in the sense that $N(t) \nrightarrow 0$ as $t \rightarrow \infty$.

\subsubsection{Linear model}

The linear model can be solved directly using the Laplace transform, but the solution obtained cannot be evaluated exactly because it contains the roots of a transcendental equation. In general it is not useful to try to compute analytical solutions to delay differential equations. However, results can be derived which provide critical insight into the behaviour of solutions of such equations.

In this subsection, we prove that the pest can go naturally extinct when the birth term is linear. For this, we need a number of preliminary results.

Lemma 3.1. If $\lambda<\mu$ in the linear model (3.5) and $N(t) \leq W$ for $t \in[m \tau,(m+1) \tau]$, where $W$ is a positive constant and $m$ is any integer $\geq-1$, then:

$$
N(t) \leq W\left(\frac{\lambda}{\mu}+\left(1-\frac{\lambda}{\mu}\right) e^{-\mu(t-(m+1) \tau)}\right) \text { for } t \in[j \tau,(j+1) \tau],
$$

for all $j \geq m+1$.

Proof. We show inductively on $j \geq(m+1)$ that (3.8) holds on the time intervals $[j \tau,(j+1) \tau]$. This type of induction, on time intervals the same width as the delay, is sometimes referred to as the method of steps [125].

Basis step $(j=m+1)$. Since $N(t) \leq W$ for $t \in[m \tau,(m+1) \tau]$, then, by (3.5), we find, for $t \in[(m+1) \tau,(m+2) \tau]$, that $\frac{d \bar{N}(t)}{d t} \leq \lambda W-\mu N(t)$.

Then (using theorem 1.1, pp. 78-79, [128]) $N(t) \leq g(t)$ on $[(m+1) \tau,(m+2) \tau]$ where $g((m+1) \tau)=W \geq N((m+1) \tau)$ and where, for $t \in[(m+1) \tau,(m+2) \tau]$, we have $\frac{d g(t)}{d t}=\lambda W-\mu g(t)$. Solving for $g(t)$ then gives the result we require on the basis step:

$$
N(t) \leq g(t)=W\left(\frac{\lambda}{\mu}+\left(1-\frac{\lambda}{\mu}\right) e^{-\mu(t-(m+1) \tau)}\right) \text { for } t \in[(m+1) \tau,(m+2) \tau]
$$

Inductive step. Assume (inductive hypothesis) for any $j \geq m+1$ that

$$
N(t) \leq W\left(\frac{\lambda}{\mu}+\left(1-\frac{\lambda}{\mu}\right) e^{-\mu(t-(m+1) \tau)}\right) \text { for } t \in[j \tau,(j+1) \tau] .
$$

Then $N(t) \leq W$ for $t \in[j \tau,(j+1) \tau]$ since $\left(1-\frac{\lambda}{\mu}\right) e^{-\mu(t-(m+1) \tau)} \leq 1-\frac{\lambda}{\mu}$ because $1-\frac{\lambda}{\mu}>0$ for $\lambda<\mu$ and $e^{-\mu(t-(m+1) \tau)} \leq 1$ for $t \geq(m+1) \tau$. Hence by (3.5):

$$
\frac{d N(t)}{d t} \leq \lambda W-\mu N(t) \text { for } t \in[(j+1) \tau,(j+2) \tau]
$$


Also by (3.10)

$$
N((j+1) \tau) \leq W\left(\frac{\lambda}{\mu}+\left(1-\frac{\lambda}{\mu}\right) e^{-\mu((j+1) \tau-(m+1) \tau)}\right) .
$$

So $N(t) \leq g(t)$ for $t \in[(j+1) \tau,(j+2) \tau]$ where

$$
g((j+1) \tau)=W\left(\frac{\lambda}{\mu}+\left(1-\frac{\lambda}{\mu}\right) e^{-\mu((j+1) \tau-(m+1) \tau)}\right) \geq N((j+1) \tau),
$$

and where

$$
\frac{d g(t)}{d t}=\lambda W-\mu g(t) \text { for } t \in[(j+1) \tau,(j+2) \tau] .
$$

Solving for $g(t)$ then gives the result we require on the inductive step:

$$
N(t) \leq g(t)=W\left(\frac{\lambda}{\mu}+\left(1-\frac{\lambda}{\mu}\right) e^{-\mu(t-(m+1) \tau)}\right) \text { for } t \in[(j+1) \tau,(j+2) \tau] .
$$

Lemma 3.2. Let $N(t)$ satisfy the linear model (3.5). Suppose $\lambda<\mu$. Also suppose $N(t) \leq W$ on $[m \tau,(m+1) \tau]$ for an integer $m \geq-1$ and a positive constant $W$. Then

$$
N(t)<\frac{1}{2} W\left(1+\frac{\lambda}{\mu}\right) \text { for } t \in[k \tau,(k+1) \tau] \text { for all } k \geq k^{*},
$$

where $k^{*}=(m+2)+\left\lfloor\frac{\ln 2}{\mu \tau}\right\rfloor$ where $\bigsqcup$ denotes the greatest integer function.

Proof. If $N(t) \leq W$ for $t \in[m \tau,(m+1) \tau]$ for an $m \geq-1$, then, by lemma 3.1,

$$
N(t) \leq W\left(\frac{\lambda}{\mu}+\left(1-\frac{\lambda}{\mu}\right) e^{-\mu(t-(m+1) \tau)}\right) \text { for } t \in[j \tau,(j+1) \tau],
$$

for all $j \geq m+1$.

As $t$ increases, $e^{-\mu(t-(m+1) \tau)}$ decreases monotonically to zero. We deduce that $e^{-\mu(t-(m+1) \tau)}<\frac{1}{2}$ on all intervals $[k \tau,(k+1) \tau]$ for which $k>k^{*}$ where $k^{*}$ is any integer for which $e^{-\mu\left(k^{*} \tau-(m+1) \tau\right)}<\frac{1}{2}$. But this last inequality can be rearranged to give $k^{*}>(m+1)+\left\lfloor\frac{\ln 2}{\mu \tau}\right\rfloor$, so we can clearly choose $k^{*}=(m+2)+\left\lfloor\frac{\ln 2}{\mu \tau}\right\rfloor$.

Then for $k \geq k^{*}$, we have, for $t \in[k \tau,(k+1) \tau]$,

$$
N(t)<W\left(\frac{\lambda}{\mu}+\left(1-\frac{\lambda}{\mu}\right)\left(\frac{1}{2}\right)\right)=\frac{1}{2} W\left(1+\frac{\lambda}{\mu}\right),
$$

where we use the fact that $1-\frac{\lambda}{\mu}>0$ for $\lambda<\mu$.

Theorem 3.1. Let $N(t)$ satisfy the linear model (3.5). If $\lambda<\mu$ and $N(t) \leq W$ on $[-\tau, 0]$ where $W$ is a positive constant, then $N(t) \rightarrow 0$ as $t \rightarrow \infty$. 
Proof. We first argue by induction on $n \geq 1$ that

$$
N(t)<\delta^{n} W \text { for } t \in[k \tau,(k+1) \tau] \text { for all } k \geq k_{n},
$$

where $\delta=\frac{1}{2}\left(1+\frac{\lambda}{\mu}\right), k_{n}=(\Omega+1)+(n-1)(\Omega+2)$, and $\Omega=\left\lfloor\frac{\ln 2}{\mu \tau}\right\rfloor$.

Basis step $(n=1)$. Since $N(t) \leq W$ for $t \in[-\tau, 0]$, then by lemma 3.2, we have:

$$
N(t)<\delta W \text { for } t \in[k \tau,(k+1) \tau] \text { for all } k \geq k_{1},
$$

where $k_{1}=(\Omega+1)$.

Inductive step. Assume (inductive hypothesis) $N(t)<\delta^{n} W$ for $t \in[k \tau,(k+1) \tau]$ for all $k \geq k_{n}$ where $k_{n}=(\Omega+1)+(n-1)(\Omega+2)$. In particular, then, $N(t)<\delta^{n} W$ for $t \in\left[k_{n} \tau,\left(k_{n}+1\right) \tau\right]$. But then, by lemma 3.2, we have:

$$
N(t)<\delta\left(\delta^{n} W\right)=\delta^{n+1} W \text { for } t \in[k \tau,(k+1) \tau] \text { for all } k \geq k_{n+1},
$$

where $k_{n+1}=\left(k_{n}+2\right)+\Omega=(\Omega+1)+n(\Omega+2)$.

This completes the induction.

Finally, to see that $N(t) \rightarrow 0$ as $t \rightarrow \infty$, we need to show that for any $\epsilon>0$, there exists $T>0$ such that, for all $t>T$, we have $N(t)<\epsilon$. (We have already seen that $N(t)$ satisfies positivity.) So, pick any $\epsilon>0$. Then there exists $n \geq 1$ such that $\delta^{n} W<\epsilon$ since $0<\delta<1$ when $\lambda<\mu$. But then, by (3.19), we have:

$$
N(t)<\delta^{n} W<\epsilon \text { for } t \in[k \tau,(k+1) \tau] \text { for all } k \geq k_{n} .
$$

In other words, $N(t)<\epsilon$ for all $t>T$ where $T=k_{n} \tau$. This completes the proof.

Although theorem 3.1 is a well known result (see example 5.1 on pages 32-33 of [95]), we have provided our own proof because it shows that $N(t)$ can be bounded above by successively smaller upper bounds as $t$ grows. We can be sure, then, that the pest numbers will not flare up temporarily before tending to zero. They cannot therefore flare up long enough for the pest to ruin a crop or cause an epidemic.

Lemmas 3.1 and 3.2 and theorem 3.1 will be useful in establishing the behaviour of $N(t)$ when $\lambda>\mu$, as we shall see in a moment. First we shall deal with the case $\lambda=\mu$. The following theorem holds:

Theorem 3.2. Assume $N(t)$ satisfies the linear model (3.5). If $\lambda=\mu$ and $M \leq N(t) \leq W$ for $t \in[-\tau, 0]$ where $M$ and $W$ are positive constants, then $M \leq N(t) \leq W$ for all $t \geq 0$.

Proof. We first prove by induction on $k \geq 0$ that $N(t) \geq M$ for $t \in[k \tau,(k+1) \tau]$.

Basis step $(k=0)$. Since $N(t) \geq M$ for $t \in[-\tau, 0]$ and $\lambda=\mu$ by assumption, then, by (3.5), we find, for $t \in[0, \tau]$, that $\frac{d N(t)}{d t} \geq \lambda M-\lambda N(t)$. 
Then $N(t) \geq g(t)$ for $t \in[0, \tau]$ where $g(0)=M \leq N(0)$ and where, for $t \in[0, \tau]$, we have $\frac{d g(t)}{d t}=\lambda M-\lambda g(t)$. Solving for $g(t)$ on $[0, \tau]$ then gives the result we require on the basis step: $N(t) \geq g(t)=M$.

Inductive step. This may be established by the same type of argument as in the basis step.

A similar induction establishes that $N(t) \leq W$ for $t \in[k \tau,(k+1) \tau]$ for $k \geq 0$. Hence $M \leq N(t) \leq W$ for $t \geq 0$.

Theorem 3.2 shows that, if $\lambda=\mu$, then the pest is endemic (if the initial data is positive) but never really becomes a more serious threat than it was in the first place. Theorem 3.2 is a stability result for it shows that population levels remain bounded.

Finally we show that for $\lambda>\mu$ then $N(t) \rightarrow \infty$ as $t \rightarrow \infty$. The method will be very similar to the method used to show that $N(t) \rightarrow 0$ when $\lambda<\mu$.

Lemma 3.3. Let $N(t)$ satisfy the linear model (3.5). If $\lambda>\mu$ and $N(t) \geq M$ for $t \in$ $[m \tau,(m+1) \tau]$, where $M$ is a positive constant and $m$ is any integer $\geq-1$, then:

$$
N(t) \geq M\left(\frac{\lambda}{\mu}+\left(1-\frac{\lambda}{\mu}\right) e^{-\mu(t-(m+1) \tau)}\right) \text { for } t \in[j \tau,(j+1) \tau],
$$

for all $j \geq m+1$.

The proof of this lemma is virtually identical to the proof of lemma 3.1, with $W$ changed to $M$ and $\leq$ signs replaced by $\geq$ signs.

Lemma 3.4. Assume $N(t)$ satisfies (3.5). Suppose $\lambda>\mu$. Also suppose $N(t) \geq M$ on $[m \tau,(m+1) \tau]$ for an integer $m \geq-1$ and a positive constant $M$. Then

$$
N(t)>\frac{1}{2} M\left(1+\frac{\lambda}{\mu}\right) \text { on }[k \tau,(k+1) \tau] \text { for all } k \geq k^{*},
$$

where $k^{*}=(m+2)+\left\lfloor\frac{\ln 2}{\mu \tau}\right\rfloor$ where $\rfloor$ denotes the greatest integer function.

The proof of this lemma is virtually identical to the proof of lemma 3.2, with $W$ changed to $M$ and < signs replaced by > signs.

Theorem 3.3. Assume $N(t)$ satisfies (3.5). If $\lambda>\mu$ and $N(t) \geq M$ for $t \in[-\tau, 0]$ where $M$ is a positive constant, then $N(t) \rightarrow \infty$ as $t \rightarrow \infty$.

Proof. An inductive argument similar to the induction in the proof of theorem 3.1 will show that, for $n \geq 1$ :

$$
N(t)>\delta^{n} M \text { for } t \in[k \tau,(k+1) \tau] \text { for all } k \geq k_{n},
$$

where $\delta=\frac{1}{2}\left(1+\frac{\lambda}{\mu}\right), k_{n}=(\Omega+1)+(n-1)(\Omega+2)$, and $\Omega=\left\lfloor\frac{\ln 2}{\mu \tau}\right\rfloor$. 
To see that $N(t) \rightarrow \infty$ as $t \rightarrow \infty$, we need to show that for any $N>0$, there exists $T>0$ such that, for all $t>T$, we have $N(t)>N$. So, pick any $N>0$. Then there exists $n \geq 1$ such that $\delta^{n} M>N$ since $\delta>1$ for $\lambda>\mu$. But then, by (3.25), we have:

$$
N(t)>\delta^{n} M>N \text { for } t \in[k \tau,(k+1) \tau] \text { for all } k \geq k_{n} .
$$

In other words, $N(t)>N$ for all $t>T$ where $T=k_{n} \tau$. The proof is complete.

In view of this last theorem, it is apparent that the linear model (3.5) is not valid indefinitely when $\lambda>\mu$. After all, populations do not tend to grow arbitrarily large in real life. Over a limited time-scale, during which the model may be valid in the case $\lambda>\mu$, we see by (3.25) that the population grows in such a way that it may be bounded below by an increasing sequence, so pest control will be warranted if these lower bounds become problematically high.

\subsubsection{Nicholson model}

Recall (equation (3.6)) that the model is:

$$
\frac{d N(t)}{d t}=\lambda_{1} N(t-\tau) e^{-\lambda_{2} N(t-\tau)}-\mu N(t) \text { for } t \geq 0,
$$

with initial data satisfying:

$$
N(t)=\phi(t) \geq 0 \text { for } t \in[-\tau, 0] .
$$

We will have occasion in this subsection to consider more specific initial data, namely:

$$
N(t)=\phi_{1}(t)>0 \text { for } t \in[-\tau, 0] \text {. }
$$

First we show that natural extinction is possible:

Theorem 3.4. Assume $N(t)$ satisfies (3.27) and (3.28). If $\lambda_{1}<\mu$ and $N(t) \leq W$ on $[-\tau, 0]$ where $W$ is a positive constant, then $N(t) \rightarrow 0$ as $t \rightarrow \infty$.

Proof. By positivity we know that $N(t-\tau) \geq 0$ for $t \geq 0$, so $e^{-\lambda_{2} N(t-\tau)} \leq 1$. But then, by (3.27), we find that

$$
\frac{d N(t)}{d t} \leq \lambda_{1} N(t-\tau)-\mu N(t) \text { for } t \geq 0
$$

Hence (using theorem 1.1, pp. 78-79, [128]) we have, for $t \geq 0$, that $N(t) \leq N_{1}(t)$, where $N_{1}(t)=N(t) \leq W$ for $t \in[-\tau, 0]$, and where, for $t \geq 0$,

$$
\frac{d N_{1}(t)}{d t}=\lambda_{1} N_{1}(t-\tau)-\mu N_{1}(t) .
$$

But then since $\lambda_{1}<\mu$, we can use theorem 3.1 to conclude that $N_{1}(t) \rightarrow 0$ (in such a way that $N_{1}(t)$ can be bounded above by a decreasing sequence) as $t \rightarrow \infty$. Since $N_{1}(t)$ is an upper bound for $N(t)$ and $N(t)$ satisfies positivity, it follows that $N(t)$ must also tend to zero. 
Now we list some results from the literature which show that the pest may persist indefinitely under certain conditions on the parameters or initial conditions.

Theorem 3.5. The solution of (3.27) and (3.29) is strictly positive and bounded for $t \geq 0$.

For a proof, see page 149 of Kuang's book [95]. This result shows that, unlike in the linear model, the population can never tend to infinity (for positive initial data).

Theorem 3.6. Let $N(t)$ satisfy (3.27) and (3.29). Assume $\lambda_{1}>\mu$, so that $\lambda_{1} N e^{-\lambda_{2} N}=\mu N$ has a unique positive equilibrium $N^{*}$. Assume $0<N(0)<N^{*}$. Then there are $\delta>0$ and $T>0$ such that, for $t>T, N(t) \geq \delta$.

For a proof, see page 161 of [95]. Roughly speaking, this result says that if the birth function is, in some sense, sufficiently large and the initial population $N(0)$ is small enough, then for all time large enough there is a positive level above which the population remains.

Before we state the next result we need to define a few terms (these terms are also defined on page 149 of [95]).

Definition 3.1. Let $N(t)$ satisfy (3.27) and (3.29). We say that a constant $N^{*}$ is globally asymptotically stable if, for fixed $\tau$, all solutions $N(t)$ tend to $N^{*}$ as $t \rightarrow \infty$. We say $N^{*}$ is absolutely globally asymptotically stable if it is globally asymptotically stable for all $\tau>0$.

Theorem 3.7. Suppose $N(t)$ satisfies (3.27) and (3.29). Assume $\lambda_{1} e^{-2}<\mu<\lambda_{1}$. Then $\lambda_{1} N e^{-\lambda_{2} N}=\mu N$ has a unique positive equilibrium $N^{*}$ and it is absolutely globally asymptotically stable.

For a proof, see corollary 9.3 on page 163 of Kuang's book [95]. In his result, Kuang effectively rescales the population by a factor $\frac{1}{\lambda_{2}}$ (we say effectively only because he uses different letters to represent the model parameters) before stating and proving for the transformed model that if $\lambda_{1} e^{-2}<\mu<\lambda_{1}$ then $N^{*}$ is absolutely globally asymptotically stable. It is easy to adapt his proof to show that the same result holds without rescaling the population, that is, it holds for the original model.

Theorem 3.8. Let $N(t)$ satisfy (3.27) and (3.29). Assume $\lambda_{1}>\mu$, so that $\lambda_{1} N e^{-\lambda_{2} N}=\mu N$ has a unique positive equilibrium $N^{*}$. Assume $\frac{1}{\lambda_{2}} \geq N^{*}$. Then $N^{*}$ is absolutely globally asymptotically stable.

For a proof, see page 164 of [95].

Theorem 3.9. Suppose $N(t)$ satisfies (3.27) and (3.29). Assume $\lambda_{1}>\mu$, so that $\lambda_{1} N e^{-\lambda_{2} N}=$ $\mu N$ has a unique positive equilibrium $N^{*}$. Assume $\frac{1}{\lambda_{2}}<N^{*}$. Then for a positive constant $\hat{f}$, which depends on the model parameters and is no bigger than $\lambda_{1} e^{-2}$, we have that $N^{*}$ is globally asymptotically stable if $\tau<\frac{1}{\mu+\hat{f}}$.

For a proof, see page 165 of [95].

Results on asymptotic stability for the Nicholson model are also given on pages 112-116 of H. Smith's book [128] for regions of the $\lambda_{1}-\mu$ parameter space, and a stability diagram for this parameter space is included on page 114. Numerical simulations in Gurney et al [82] suggest that stable sustained oscillations exist for some regions of the $\lambda_{1}-\mu$ parameter space. Such oscillations are not surprising - the birth function in the Nicholson model is a negative feedback term with a delay, but systems with terms of this kind are known analytically to lead to oscillations [130]. 


\subsubsection{Allee model}

To gain an idea of what results to look for in the Allee model (equation (3.7)), we consider first what happens in the absence of delay:

Lemma 3.5. In the model

$$
\frac{d N(t)}{d t}=\alpha_{1}(N(t))^{2} e^{-\alpha_{2} N(t)}-\mu N(t) \text { for } t \geq 0
$$

where $N(0)>0$, then $N(t)>0$ for all $t>0$ and $N=0$ is always a fixed point. Also:

1) if $\alpha_{1}<e \alpha_{2} \mu$ then there are no positive fixed points and $N(t) \rightarrow 0$ as $t \rightarrow \infty$

2) if $\alpha_{1}=e \alpha_{2} \mu$ then there is one positive fixed point $N_{1}=\frac{1}{\alpha_{2}}$ and it is semi-stable. If $N(0)<\frac{1}{\alpha_{2}}$ then $N(t) \rightarrow 0$ as $t \rightarrow \infty$ but if $N(0)>\frac{1}{\alpha_{2}}$ then $N(t) \rightarrow N_{1}=\frac{1}{\alpha_{2}}$

3) if $\alpha_{1}>e \alpha_{2} \mu$ then there are two positive fixed points, $N_{1}$ and $N_{2}$, where $0<N_{1}<N_{2}$, and where $N_{1}$ is unstable and $N_{2}$ is stable. If $N(0)<N_{1}$ then $N(t) \rightarrow 0$ as $t \rightarrow \infty$ but if $N(0)>N_{1}$ then $N(t) \rightarrow N_{2}$.

The proof is trivial. Guided by this lemma, we now find extinction and endemicity results for the Allee model (equation (3.7)). For ease of reference, we repeat the model here:

$$
\frac{d N(t)}{d t}=\alpha_{1}(N(t-\tau))^{2} e^{-\alpha_{2} N(t-\tau)}-\mu N(t) \text { for } t \geq 0,
$$

and we shall assume the initial data satisfies:

$$
0 \leq N(t)=\phi(t) \leq W \text { for } t \in[-\tau, 0],
$$

where $W$ is a positive constant.

Theorem 3.10. Let $N(t)$ satisfy (3.33) and (3.34). If $\alpha_{1}<e \alpha_{2} \mu$, then $N(t) \rightarrow 0$ as $t \rightarrow \infty$.

Proof. By assumption $\alpha_{1}<e \alpha_{2} \mu$. It follows that $\alpha_{1}<e \alpha_{2} \alpha$ where $\alpha=\frac{1}{2}\left(\mu+\frac{\alpha_{1}}{\alpha_{2} e}\right)<$ $\mu$. But it is trivial to show that if $\alpha_{1}<e \alpha_{2} k$ for some $k>0$, then $\alpha_{1} N^{2} e^{-\alpha_{2} N}<k N$ for $N>0$. So certainly for $\alpha=\frac{1}{2}\left(\mu+\frac{\alpha_{1}}{\alpha_{2} e}\right)$ we have

$$
\alpha_{1} N^{2} e^{-\alpha_{2} N}<\alpha N \text { for } N>0 \text {. }
$$

By positivity we know that $N(t-\tau) \geq 0$ for $t \geq 0$, so $e^{-\alpha_{2} N(t-\tau)} \leq 1$. But then, by (3.33) and (3.35), we find that

$$
\frac{d N(t)}{d t} \leq \alpha N(t-\tau)-\mu N(t) \text { for } t \geq 0
$$

where $0<\alpha<\mu$.

Hence (using theorem 1.1, pp. 78-79, [128]), we have, for $t \geq 0$, that $N(t) \leq N_{1}(t)$, where $N_{1}(t)=N(t) \leq W$ for $t \in[-\tau, 0]$ and where, for $t \geq 0$,

$$
\frac{d N_{1}(t)}{d t}=\alpha N_{1}(t-\tau)-\mu N_{1}(t)
$$


But then since $\alpha<\mu$, we can use theorem 3.1 to conclude that $N_{1}(t) \rightarrow 0$ (in such a way that $N_{1}(t)$ can be bounded above by a decreasing sequence) as $t \rightarrow \infty$. Since $N_{1}(t)$ is an upper bound for $N(t)$ and $N(t)$ satisfies positivity, it follows that $N(t)$ must also tend to zero.

The next result shows that, as in the Nicholson model and unlike in the linear model, the population can never tend to infinity in the Allee model.

Theorem 3.11. Let $N(t)$ satisfy (3.33) and (3.34). Then $N(t)$ is bounded for all $t \geq 0$.

Proof. We know that $N(t)$ satisfies positivity for $t \geq 0$ (section 3.2). Also, $N(t)$ satisfies positivity initially - by equation (3.34), we know that $N(t) \geq 0$ for $t \in[-\tau, 0]$. Hence $N(t) \geq 0$ for $t \geq-\tau$. But then $\alpha_{1}(N(t-\tau))^{2} e^{-\alpha_{2} N(t-\tau)}$ is bounded above for $t \geq 0$, since the function $b(N)=\alpha_{1} N^{2} e^{-\alpha_{2} N}$ is bounded above if $N \geq 0$. In fact, if $N \geq 0$, we trivially find that $b(N) \leq b\left(\frac{2}{\alpha_{2}}\right)=\alpha_{1}\left(\frac{2}{\alpha_{2}}\right)^{2} e^{-2}$. For ease of notation, refer to this upper bound for $b(N)$ as $K$. Then, by (3.33), we see that:

$$
\frac{d N(t)}{d t} \leq K-\mu N(t) \text { for } t \geq 0 .
$$

Hence $N(t) \leq N_{1}(t)$ for $t \geq 0$ where $N_{1}(0)=W \geq N(0)$ (using equation (3.34)) and where $\frac{d N_{1}(t)}{d t}=K-\mu N_{1}(t)$ for $t \geq 0$. Solving for $N_{1}(t)$ reveals that

$$
N(t) \leq N_{1}(t)=\frac{K}{\mu}+\left(W-\frac{K}{\mu}\right) e^{-\mu t} \text { for } t \geq 0 .
$$

Therefore $N(t) \leq \max \left(\frac{K}{\mu}, W\right)$ for $t \geq 0$, so certainly $N(t)$ is bounded above, as required.

The fixed points for the Allee model (equation (3.33)) are the same as the fixed points for the model without delay (equation (3.32)). Henceforth define $N_{1}$ and $N_{2}$ as in lemma 3.5. From result 3 ) in lemma 3.5 we know, for the model without delay, that if $\alpha_{1}>e \alpha_{2} \mu$ and the initial condition $N(0)$ is greater than $N_{1}$, then $N(t)>N_{1}$ for all $t>0$. The analogous result for the Allee model does not hold, as the next lemma reveals.

Lemma 3.6. Let $N(t)$ satisfy (3.33). Suppose $\alpha_{1}>e \alpha_{2} \mu$. Let $N_{1}$ and $N_{2}$ be defined as in result 3) of lemma 3.5. Also suppose $N(t) \in\left[C_{1}, C_{2}\right]$ for $t \in[-\tau, 0]$ where $C_{1}$ and $C_{2}$ are constants and $C_{1}>N_{2}$. Then, for $C_{1}$ and $\tau$ large enough, $N(\tau)<N_{1}$.

Proof. It is routine to verify that the birth function $b(N)=\alpha_{1} N^{2} e^{-\alpha_{2} N}$ is monotonic decreasing for $N>\max \left(N_{2}, \frac{2}{\alpha_{2}}\right)$, where it is trivial to see that $\frac{2}{\alpha_{2}}$ is the unique value of $N \geq 0$ such that $b(N)$ is maximum. But then, if $C_{1}>\max \left(N_{2}, \frac{2}{\alpha_{2}}\right)$, we can say that $b(N(t)) \leq b\left(C_{1}\right)$ for $t \in[-\tau, 0]$. Hence by (3.33):

$$
\frac{d N(t)}{d t} \leq b\left(C_{1}\right)-\mu N(t) \text { for } t \in[0, \tau] .
$$


Then $N(t) \leq g(t)$ on $[0, \tau]$ where $g(0)=C_{2} \geq N(0)$ and where

$$
\frac{d g(t)}{d t}=b\left(C_{1}\right)-\mu g(t) \text { for } t \in[0, \tau] .
$$

Solving for $g(t)$ then gives:

$$
N(t) \leq g(t)=\frac{b\left(C_{1}\right)}{\mu}+\left(C_{2}-\frac{b\left(C_{1}\right)}{\mu}\right) e^{-\mu t} \text { for } t \in[0, \tau] .
$$

We then have:

$$
N(\tau) \leq \frac{b\left(C_{1}\right)}{\mu}+\left(C_{2}-\frac{b\left(C_{1}\right)}{\mu}\right) e^{-\mu \tau}
$$

Now choose $C_{1}$ large enough that $\frac{b\left(C_{1}\right)}{\mu}<\frac{1}{2} N_{1}$. This is possible since $b(N) \rightarrow 0$ as $N \rightarrow \infty$. Then, since $-\frac{b\left(C_{1}\right)}{\mu}<0$, we can write:

$$
N(\tau) \leq \frac{1}{2} N_{1}+C_{2} e^{-\mu \tau}
$$

Now choose $\tau$ large enough that $C_{2} e^{-\mu \tau}<\frac{1}{2} N_{1}$. We can do this because $\tau$ is independent of $C_{2}$ and $C_{1}$. Then $N(\tau)<N_{1}$, as required.

Now we prove a lemma which will help us show that the pest may persist indefinitely under certain conditions on the parameters and initial conditions:

Lemma 3.7. 1) Let $b(N)=\alpha_{1} N^{2} e^{-\alpha_{2} N}$. Then $b(N)$ has a unique maximum for $N \geq 0$, which occurs where $N=\frac{2}{\alpha_{2}}$. For $N<\frac{2}{\alpha_{2}}$ then $b(N)$ is monotonic increasing, and for $N>\frac{2}{\alpha_{2}}$ then $b(N)$ is monotonic decreasing.

2) Suppose $\frac{2}{\alpha_{2}}<L<U<\frac{3}{\alpha_{2}}$ for some $L$ and $U$. Then $\frac{b(L)}{U}<\frac{b(U)}{L}$.

Proof. The first part is trivial. To prove 2), we begin by defining $h(N)=N b(N)=$ $\alpha_{1} N^{3} e^{-\alpha_{2} N}$. We find that:

$$
\frac{d h(N)}{d N}=\alpha_{1} N^{2} e^{-\alpha_{2} N}\left(3-\alpha_{2} N\right)
$$

It follows that $h(N)$ has a single turning point for $N \geq 0$, and this occurs where $N=\frac{3}{\alpha_{2}}$. For $\frac{2}{\alpha_{2}}<N<\frac{3}{\alpha_{2}}$, it is clear that $\frac{d h(N)}{d N}>0$, so that $h(N)$ is monotonic increasing. Hence if $\frac{2}{\alpha_{2}}<L<U<\frac{3}{\alpha_{2}}$, we must have $h(L)<h(U)$, which can be rewritten as $\frac{b(L)}{U}<\frac{b(U)}{L}$ since $h(N)=N b(N)$.

Using this lemma, we can now prove the following theorem:

Theorem 3.12. Suppose $N(t)$ satisfies (3.33). Let $b(N)=\alpha_{1} N^{2} e^{-\alpha_{2} N}$. Assume the following:

1) L and $U$ satisfy $\frac{2}{\alpha_{2}}<L<U<\frac{3}{\alpha_{2}}$ 
2) $N(t) \in[L, U]$ for $t \in[-\tau, 0]$

3) $\frac{b(L)}{U}<\mu<\frac{b(U)}{L}$.

Then $N(t) \in[L, U]$ for all $t \geq 0$.

Proof. Note that assumption 1) and lemma 3.7 make assumption 3) sensible, that is, it can actually hold.

The proof is by induction on the time intervals $[j \tau,(j+1) \tau]$ for $j \geq 0$.

Basis step $(j=0)$. Notice first of all by part 1$)$ of lemma 3.7 that $b(N)$ is monotonic decreasing for $N>\frac{2}{\alpha_{2}}$. Then, using assumptions 1) and 2), $b(N(t)) \geq b(U)$ for $t \in$ $[-\tau, 0]$. Hence by equation (3.33):

$$
\frac{d N(t)}{d t} \geq b(U)-\mu N(t) \text { for } t \in[0, \tau] .
$$

Then $N(t) \geq g(t)$ for $t \in[0, \tau]$ where $g(0)=L \leq N(0)$ and where:

$$
\frac{d g(t)}{d t}=b(U)-\mu g(t) \text { for } t \in[0, \tau] .
$$

Solving for $g(t)$ then gives:

$$
N(t) \geq g(t)=\frac{b(U)}{\mu}+\left(L-\frac{b(U)}{\mu}\right) e^{-\mu t} \text { for } t \in[0, \tau] .
$$

By assumption 3), we can write $L-\frac{b(U)}{\mu}<0$. It follows that the lower bound for $N(t)$ in (3.48) is increasing on $[0, \tau]$. Since the lower bound begins at $t=0$ with the value $L$ and then increases, it must be bounded below by $L$ on the whole interval. Hence $N(t) \geq L$ for $t \in[0, \tau]$. This completes the first half of the basis step.

Next we need $N(t) \leq U$ on $[0, \tau]$. Again, by part 1) of lemma 3.7, we know that $b(N)$ is monotonic decreasing for $N>\frac{2}{\alpha_{2}}$. Then, using assumptions 1) and 2), $b(N(t)) \leq b(L)$ for $t \in[-\tau, 0]$. Hence by equation (3.33):

$$
\frac{d N(t)}{d t} \leq b(L)-\mu N(t) \text { for } t \in[0, \tau]
$$

But then $N(t) \leq g(t)$ for $t \in[0, \tau]$ where $g(0)=U \geq N(0)$ and where:

$$
\frac{d g(t)}{d t}=b(L)-\mu g(t) \text { for } t \in[0, \tau] .
$$

Solving for $g(t)$ then gives:

$$
N(t) \leq g(t)=\frac{b(L)}{\mu}+\left(U-\frac{b(L)}{\mu}\right) e^{-\mu t} \text { for } t \in[0, \tau] .
$$

By assumption 3), $\mu>\frac{b(L)}{U}$, which may be rewritten as $U-\frac{b(L)}{\mu}>0$. It follows that the upper bound for $N(t)$ in (3.51) is decreasing on $[0, \tau]$. Since the upper bound begins at $t=0$ with the value $U$ and then decreases, it must be bounded above by $U$ on the whole interval. Hence $N(t) \leq U$ for $t \in[0, \tau]$. This completes the basis step.

Inductive step. This is very similar to the basis step. 
Finally we show that if the initial data is sufficiently small, then the population must go extinct. Since extinction is inevitable when $\mu>\frac{\alpha_{1}}{\alpha_{2} e}$ by theorem 3.10, we need only consider the case $\mu \leq \frac{\alpha_{1}}{\alpha_{2} e}$, an inequality that will be assumed for the remainder of this subsection. If this is assumed in the model with no delay, then by results 2 ) and 3 ) in lemma 3.5, there is a positive constant $N_{1}$ such that if $N(0)<N_{1}$, then $N(t) \rightarrow 0$ as $t \rightarrow \infty$. Here $N_{1}$ is the least positive solution to

$$
\alpha_{1} N^{2} e^{-\alpha_{2} N}-\mu N=0 .
$$

When delay is introduced into the model, this same number $N_{1}$, the least positive solution to (3.52), will satisfy a similar condition, namely that when $N(t)<N_{1}$ for $t \in[-\tau, 0]$, then $N(t) \rightarrow 0$ as $t \rightarrow \infty$. Before we can prove this we must state and prove a number of lemmas:

Lemma 3.8. Let $b(N)=\alpha_{1} N^{2} e^{-\alpha_{2} N}$ and assume $\mu \leq \frac{\alpha_{1}}{\alpha_{2} e}$. Let $N_{1}$ be defined as in results 2) and 3) of lemma 3.5. If $0<R<N_{1}$, then $b(R)<\mu R$.

Proof. This is obvious geometrically but we offer an analytic argument for the more rigorous reader.

Define the function $f(N)=b(N)-\mu N$. Then $f(0)=0$ and $f\left(N_{1}\right)=0$ where $N_{1}$ is the least positive root, which exists since $\mu \geq \frac{\alpha_{1}}{\alpha_{2} e}$. Also, $f(N)$ is continuous and it is straightforward to check that $\left.\frac{d f}{d N}\right|_{N=0}<0$. It follows that $f(N)<0$ for $0<N<N_{1}$.

Lemma 3.9. Suppose $N(t)$ satisfies (3.33). Let $b(N)=\alpha_{1} N^{2} e^{-\alpha_{2} N}$ and assume $\mu \leq \frac{\alpha_{1}}{\alpha_{2} e}$. Let $N_{1}$ be defined as in results 2) and 3) of lemma 3.5. If $N(t) \leq R$ for $t \in[m \tau,(m+1) \tau]$, where $R$ is a constant such that $0<R<N_{1}$, and $m$ is an integer $\geq-1$, then for all integers $j \geq m+1$ we have

$$
N(t) \leq \frac{b(R)}{\mu}+\left(R-\frac{b(R)}{\mu}\right) e^{-\mu(t-(m+1) \tau)} \text { for } t \in[j \tau,(j+1) \tau] .
$$

Proof. The proof is by induction on $j \geq m+1$.

Basis step $(j=m+1)$. It is trivially verified that $N_{1}<\frac{2}{\alpha_{2}}$ where $\frac{2}{\alpha_{2}}$ is the value of $N>0$ for which $b(N)$ is maximum. But then, by part 1) of lemma 3.7, the birth function $b(N)=\alpha_{1} N^{2} e^{-\alpha_{2} N}$ is monotonic increasing for $N$ satisfying $0<N<N_{1}$. But then, since $N(t) \leq R<N_{1}$ for $t \in[m \tau,(m+1) \tau]$, we must have $b(N(t)) \leq b(R)$ on this interval, so that $b(N(t-\tau)) \leq b(R)$ for $t \in[(m+1) \tau,(m+2) \tau]$. Hence using equation (3.33), $\frac{d N(t)}{d t} \leq b(R)-\mu N(t)$ for $t \in[(m+1) \tau,(m+2) \tau]$.

By assumption $N(t) \leq R$ for $t \in[m \tau,(m+1) \tau]$, so certainly $N((m+1) \tau) \leq R$. Therefore $N(t) \leq g(t)$ for $t \in[(m+1) \tau,(m+2) \tau]$ where $g((m+1) \tau)=R \geq N((m+1) \tau)$ and where $\frac{d g(t)}{d t}=b(R)-\mu g(t)$ for $t \in[(m+1) \tau,(m+2) \tau]$. Solving for $g(t)$, we find the result required on the basis step:

$$
N(t) \leq g(t)=\frac{b(R)}{\mu}+\left(R-\frac{b(R)}{\mu}\right) e^{-\mu(t-(m+1) \tau)} \text { on }[(m+1) \tau,(m+2) \tau] .
$$


Inductive step. Assume (inductive hypothesis) for some $j \geq m+1$ that

$$
N(t) \leq \frac{b(R)}{\mu}+\left(R-\frac{b(R)}{\mu}\right) e^{-\mu(t-(m+1) \tau)} \text { for } t \in[j \tau,(j+1) \tau] .
$$

Now by lemma 3.8, we know that $b(R)<\mu R$ for $0<R<N_{1}$. In other words, $R-\frac{b(R)}{\mu}>0$, so that the upper bound in (3.55) is decreasing on $[j \tau,(j+1) \tau]$. Then, since the upper bound for $N(t)$ in (3.55) is $R$ when $t=j \tau$, it follows that

$$
N(t) \leq R \text { for } t \in[j \tau,(j+1) \tau] .
$$

But then, since $b(N)$ is monotonic increasing for $N$ such that $0<N<N_{1}$, and since $0<R<N_{1}$, we must have, by (3.56), that $b(N(t)) \leq b(R)$ for $t \in[j \tau,(j+1) \tau]$, which gives $b(N(t-\tau)) \leq b(R)$ for $t \in[(j+1) \tau,(j+2) \tau]$. Hence by equation (3.33):

$$
\frac{d N(t)}{d t} \leq b(R)-\mu N(t) \text { for } t \in[(j+1) \tau,(j+2) \tau]
$$

Also, by (3.55), we can write:

$$
N((j+1) \tau) \leq \frac{b(R)}{\mu}+\left(R-\frac{b(R)}{\mu}\right) e^{-\mu((j+1) \tau-(m+1) \tau)} .
$$

It follows that $N(t) \leq g(t)$ for $t \in[(j+1) \tau,(j+2) \tau]$ where

$$
g((j+1) \tau)=\frac{b(R)}{\mu}+\left(R-\frac{b(R)}{\mu}\right) e^{-\mu((j+1) \tau-(m+1) \tau)} \geq N((j+1) \tau),
$$

and

$$
\frac{d g(t)}{d t}=b(R)-\mu g(t) \text { for } t \in[(j+1) \tau,(j+2) \tau] .
$$

Solving for $g(t)$, we find the result required on the inductive step:

$$
N(t) \leq g(t)=\frac{b(R)}{\mu}+\left(R-\frac{b(R)}{\mu}\right) e^{-\mu(t-(m+1) \tau)} \text { on }[(j+1) \tau,(j+2) \tau] .
$$

Lemma 3.10. Suppose $N(t)$ satisfies (3.33). Let $b(N)=\alpha_{1} N^{2} e^{-\alpha_{2} N}$ and assume $\mu \leq \frac{\alpha_{1}}{\alpha_{2} e}$. Let $N_{1}$ be defined as in results 2) and 3) of lemma 3.5. Suppose $N(t) \leq R<N_{1}$ for $t \in$ $[m \tau,(m+1) \tau]$ for an integer $m \geq-1$ and where $R$ is a positive constant. Then

$$
N(t)<\frac{1}{2}\left(R+\frac{b(R)}{\mu}\right) \text { for } t \in[k \tau,(k+1) \tau] \text { for all } k \geq k^{*},
$$

where $k^{*}=(m+2)+\left\lfloor\frac{\ln 2}{\mu \tau}\right\rfloor$ where $\bigsqcup$ denotes the greatest integer function.

Proof. If $N(t) \leq R<N_{1}$ for $t \in[m \tau,(m+1) \tau]$ for an $m \geq-1$, then, by lemma 3.9, we have, for all $k \geq m+1$, that

$$
N(t) \leq \frac{b(R)}{\mu}+\left(R-\frac{b(R)}{\mu}\right) e^{-\mu(t-(m+1) \tau)} \text { on }[k \tau,(k+1) \tau] .
$$


As $t$ increases, $e^{-\mu(t-(m+1) \tau)}$ decreases monotonically to zero. Hence $e^{-\mu(t-(m+1) \tau)}<$ $\frac{1}{2}$ on all intervals $[k \tau,(k+1) \tau]$ for which $k \geq k^{*}$ where $k^{*}$ is any integer for which $e^{-\mu\left(k^{*} \tau-(m+1) \tau\right)}<\frac{1}{2}$. But this last inequality may be rearranged to give $k^{*}>(m+$ 1) $+\left\lfloor\frac{\ln 2}{\mu \tau}\right\rfloor$, so we can clearly choose $k^{*}=(m+2)+\left\lfloor\frac{\ln 2}{\mu \tau}\right\rfloor$.

But then, since $R-\frac{b(R)}{\mu}>0$ by lemma 3.8, we have for $t \in[k \tau,(k+1) \tau]$ where $k \geq k^{*}$, that $\left(R-\frac{b(R)}{\mu}\right) e^{-\mu(t-(m+1) r)}<\frac{1}{2}\left(R-\frac{b(R)}{\mu}\right)$. Hence, for $t \in[k \tau,(k+1) \tau]$ for $k \geq k^{*}$,

$$
N(t) \leq \frac{b(R)}{\mu}+\left(R-\frac{b(R)}{\mu}\right) e^{-\mu(t-(m+1) \tau)}<\frac{1}{2}\left(R+\frac{b(R)}{\mu}\right) .
$$

Definition 3.2. Let $b(N)=\alpha_{1} N^{2} e^{-\alpha_{2} N}$ and assume $\mu \leq \frac{\alpha_{1}}{\alpha_{2} e}$. Let $N_{1}$ be defined as in results 2) and 3) of lemma 3.5. Define the sequence $R_{n}$ where $R_{0}$ is some given initial value satisfying $0<R_{0}<N_{1}$ and

$$
R_{n}=\frac{1}{2}\left(R_{n-1}+\frac{b\left(R_{n-1}\right)}{\mu}\right) \text { for } n \geq 1 .
$$

Lemma 3.11. $R_{n} \rightarrow 0$ as $n \rightarrow \infty$.

Proof. First we show by induction that the $R_{n}$ form a decreasing sequence bounded below by zero.

Basis step. Now $R_{0}>0$ by assumption and $R_{1}=\frac{1}{2}\left(R_{0}+\frac{b\left(R_{0}\right)}{\mu}\right)>0$ since $b(N)>0$ for $N>0$.

Also $R_{1}=\frac{1}{2}\left(R_{0}+\frac{b\left(R_{0}\right)}{\mu}\right)<\frac{1}{2}\left(R_{0}+R_{0}\right)=R_{0}$, since $\frac{b\left(R_{0}\right)}{\mu}<R_{0}$ by lemma 3.8 and the fact that $0<R_{0}<N_{1}$.

Inductive step. Assume that $0<R_{n}<R_{n-1}<R_{n-2}<\cdots<R_{0}$. Then $R_{n+1}=$ $\frac{1}{2}\left(R_{n}+\frac{b\left(R_{n}\right)}{\mu}\right)>0$ since $b(N)>0$ for $N>0$.

Also $R_{n+1}=\frac{1}{2}\left(R_{n}+\frac{b\left(R_{n}\right)}{\mu}\right)<\frac{1}{2}\left(R_{n}+R_{n}\right)=R_{n}$, since $\frac{b\left(R_{n}\right)}{\mu}<R_{n}$ by lemma 3.8, the inductive assumption that $0<R_{n}<R_{0}$, and the fact that $R_{0}<N_{1}$. This completes the induction.

Hence the $R_{n}$ are a decreasing sequence, bounded below by zero. Then $R_{n} \rightarrow$ some limit $R^{*}$ where $0 \leq R^{*}<N_{1}$ and where $R^{*}$ satisfies (because $b(N)$ is a continuous function of $N$ )

$$
R^{*}=\frac{1}{2}\left(R^{*}+\frac{b\left(R^{*}\right)}{\mu}\right)
$$

Hence $R^{*}=\frac{b\left(R^{*}\right)}{\mu}$, which implies that $R^{*}=0$ since this is the only solution for which $0 \leq R^{*}<N_{1}$ (in results 2) and 3) of lemma 3.5, $N_{1}$ is defined as the least positive solution to $b(N)=\mu N)$.

Finally, we can state and prove our theorem: 
Theorem 3.13. Suppose $N(t)$ satisfies (3.33). Let $b(N)=\alpha_{1} N^{2} e^{-\alpha_{2} N}$ and assume $\mu \leq$ $\frac{\alpha_{1}}{\alpha_{2} e}$. Let $N_{1}$ be defined as in results 2) and 3) of lemma 3.5. If $N(t) \leq R_{0}$ on $[-\tau, 0]$ where $0<R_{0}<N_{1}$, then $N(t) \rightarrow 0$ as $t \rightarrow \infty$.

Proof. We first argue by induction on $n \geq 1$ that

$$
N(t)<R_{n} \text { on }[k \tau,(k+1) \tau] \text { for all } k \geq k_{n},
$$

where $k_{n}=(\Omega+1)+(n-1)(\Omega+2)$ where $\Omega=\left\lfloor\frac{\ln 2}{\mu \tau}\right\rfloor$.

Basis step $(n=1)$. Since $N(t)<R_{0}$ for $t \in[-\tau, 0]$, then by lemma 3.10, and using the definition of the sequence $R_{n}$, we have:

$$
N(t)<R_{1} \text { for } t \in[k \tau,(k+1) \tau] \text { for all } k \geq k_{1},
$$

where $k_{1}=(\Omega+1)$.

Inductive step. Assume (inductive hypothesis) for some $n \geq 1$ that $N(t)<R_{n}$ for $t \in[k \tau,(k+1) \tau]$ for all $k \geq k_{n}$ where $k_{n}=(\Omega+1)+(n-1)(\Omega+2)$. In particular, then, $N(t)<R_{n}$ for $t \in\left[k_{n} \tau,\left(k_{n}+1\right) \tau\right]$. But then, by lemma 3.10, and using the definition of the sequence $R_{n}$, we have:

$$
N(t)<R_{n+1} \text { for } t \in[k \tau,(k+1) \tau] \text { for all } k \geq k_{n+1},
$$

where $k_{n+1}=\left(k_{n}+2\right)+\Omega=(\Omega+1)+n(\Omega+2)$.

This completes the induction establishing (3.67).

Finally, pick any $\epsilon>0$. Then there exists $n \geq 1$ such that $R_{n}<\epsilon$ by lemma 3.11. But then by (3.67), we have:

$$
N(t)<R_{n}<\epsilon \text { for all } t>k_{n} \tau=((\Omega+1)+(n-1)(\Omega+2)) \tau=T .
$$

In other words, for any $\epsilon>0$, we have found a $T>0$ such that for all $t>T$, then $N(t)<\epsilon$. Since we have already seen that $N(t)$ satisfies positivity, we must conclude that $N(t) \rightarrow 0$ as $t \rightarrow \infty$.

From theorem 3.13 we notice, appropriately enough, that an Allee effect holds in the Allee model (equation (3.33)).

\subsection{Culling regimes}

We now turn our attention to the construction of successful adult impulsive culling regimes for our models. (We have commented on why such a type of regime may be an appropriate control method in section 2.1.) A culling regime will be considered successful if it guarantees eradication of the pest population, which will certainly occur if the adult population $N(t)$ tends to zero as $t \rightarrow \infty$. We concede that words 
such as "eradication" are becoming less popular and that the emphasis nowadays is on sustainable control methods (see section 2.3).

The existence and uniqueness of solutions is preserved when an impulsive culling regime (as described in definition 3.3 below) is imposed on our three models. As in section 3.2, we refer the reader to Kuang's book [95] for existence and uniqueness results. Notice that positivity is trivially preserved when impulsive culling is introduced into our models (proposition 2.1, [125]).

We begin by constructing a successful culling regime for the linear model in subsection 3.4.1 and then we adapt it to the other models (subsections 3.4.2 and 3.4.3). Of particular significance is the calculation of an explicit finite upper bound for the number of culls needed to guarantee extinction in the Allee model (subsection 3.4.3). Culling regimes for the linear model have already been considered by Simons and Gourley [125], whose methods differ from ours and whose regime is not proven with complete rigour to succeed.

\subsubsection{Linear model}

By theorem 3.1, we know that when $\lambda<\mu$ then $N(t) \rightarrow 0$ as $t \rightarrow \infty$ and that $N(t)$ will be bounded above by successively smaller upper bounds. But by theorem 3.3, $N(t) \rightarrow \infty$ when $\lambda>\mu$, and by theorem 3.2 there is also endemicity when $\lambda=\mu$. So pest control is only warranted when $\lambda \geq \mu$, a relationship that will be assumed to hold for the remainder of this subsection.

Definition 3.3. Let culls occur at times $t_{i}$ for $i \geq 1$. The cull occurring at time $t_{i}$ is said to be the $i$-th cull. Assume $t_{1}>0$ and that $0<t_{i+1}-t_{i} \leq \tau$ for $i \geq 1$. Also assume $t_{i} \rightarrow \infty$ as $i \rightarrow \infty$. At the $i$-th cull the population is scaled by a factor $b_{i}$ where $0 \leq b_{i}<1$. (So we assume all culls kill at least some adults.) Thus:

$$
N\left(t_{i}\right)=b_{i} N\left(t_{i}^{-}\right) \text {for } i \geq 1,
$$

where $t_{i}^{-}$is the time "momentarily" before $t_{i}$.

Between culls the adult population $N(t)$ satisfies (3.5). Thus:

$$
\frac{d N(t)}{d t}=\lambda N(t-\tau)-\mu N(t) \text { for } t \geq 0, t \neq t_{i} \text { where } i \geq 1 .
$$

Equation (3.72) is, however, assumed to hold at $t_{i}$ in the sense of left and right differentiability, though of course the left and right derivatives at $t_{i}$ need not be the same.

After collecting data for a real-world pest population, it will take time before a decision can be made on how best to control it. Precisely how much time it will take may be difficult to predict, which is why we have made no restriction on the size of $t_{1}$. The restriction that $t_{i+1}-t_{i} \leq \tau$ for $i \geq 1$ is made because it enables a relatively simple analysis to be carried out. Insect maturation ages can vary from a few days to 17 years (p. 58, [117]) but for agricultural pests they are typically of the order of magnitude of a week or two [82]. Tropical insect pests can usually breed faster than temperate insects by virtue of the warmer climate. The restriction of having to apply a pesticide at least once a week will not be unachievable for a big commercial farm. For mosquitoes the maturation age is around one to two weeks [8]. The assumption that $t_{i} \rightarrow \infty$ as $i \rightarrow \infty$ is necessary because it is impossible to perform infinitely many culls in a finite time.

The following subsequence will be needed in our later analysis: 
Definition 3.4. Define a subsequence of the times $t_{i}$ as $t_{i_{n}}$ for $n \geq 1$, where $t_{i_{1}}=t_{1}$ and $t_{i_{n+1}}$ is the least $t_{i}$ to be bigger than or equal to $t_{i_{n}}+\tau$.

It should be understood that in the subsequence $t_{i_{n}}, n \geq 1$, the " $i$ " is fixed and is included only to remind us that the $t_{i_{n}}$ are a subsequence of the culling times $t_{i}$. Since $t_{i} \rightarrow \infty$ as $i \rightarrow \infty$, it is trivially true that $t_{i_{n}} \rightarrow \infty$ as $n \rightarrow \infty$.

Certain quantities will be useful to us in our analysis. Define the following:

$$
\begin{aligned}
\delta_{i} & =\frac{\lambda}{\mu}+e^{-\mu\left(t_{i+1}-t_{i}\right)}\left(b_{i}-\frac{\lambda}{\mu}\right) \text { for } i \geq 1 \\
\Delta & =\sup _{i \geq 1} \delta_{i} \\
b & =\sup _{i \geq 1} b_{i} \\
T & =\sup _{i \geq 1}\left(t_{i+1}-t_{i}\right) \leq \tau
\end{aligned}
$$
proofs:

The following lemma will serve as a useful shortcut in the details of subsequent

Lemma 3.12. Suppose $N(t)$ satisfies (3.72). Assume $N(t-\tau) \leq U$ when $t \in[A, B]$ where $0 \leq A<B \leq A+\tau$. Assume also that $N(A) \leq \xi$ and that no culls occur in the interval $(A, B)$.

Then, for $t \in[A, B]$, we have:

$$
N(t) \leq \frac{\lambda}{\mu} U+e^{-\mu(t-A)}\left(\xi-\frac{\lambda}{\mu} U\right) .
$$

In particular, if $\xi=\gamma U$, then

$$
N(t) \leq U\left[\frac{\lambda}{\mu}+e^{-\mu(t-A)}\left(\gamma-\frac{\lambda}{\mu}\right)\right] .
$$

Proof. First notice by equation (3.72) that

$$
\frac{d N(t)}{d t} \leq \lambda U-\mu N(t) \text { for } t \in[A, B) .
$$

Inequality (3.79) holds at $t=A$ in the sense of left differentiability.

Then $N(t) \leq g(t)$ for $t \in[A, B)$ where $g(A)=\xi \geq N(A)$ and

$$
\frac{d g(t)}{d t}=\lambda U-\mu g(t) \text { for } t \in[A, B) \text {. }
$$

Solving for $g(t)$ gives:

$$
N(t) \leq g(t)=\frac{\lambda}{\mu} U+e^{-\mu(t-A)}\left(\xi-\frac{\lambda}{\mu} U\right) \text { for } t \in[A, B) .
$$

In particular this holds for $t=B^{-}$, the time just before $t=B$. If a cull occurs at time $t=B$ with intensity $b_{i}$, say, we will obtain $N(B)=b_{i} N\left(B^{-}\right) \leq N\left(B^{-}\right)$since $0<b_{i}<1$. Hence the inequality (3.81) will hold on $[A, B]$. If there is no cull at $t=B$ 
then $N(t)$ will be continuous at $t=B$, so that $N(B)=N\left(B^{-}\right)$, and inequality (3.81) will again hold on $[A, B]$. Inequality (3.78) follows trivially from setting $\xi=\gamma U$ in inequality (3.77).

The next lemma acts as a sort of initial condition for the theorem which follows.

Lemma 3.13. Suppose $N(t)$ satisfies (3.72) and that $N(t) \leq W$ for $t \in[-\tau, 0]$ where $W$ is a positive constant. Assume $\lambda \geq \mu$. For $t \in\left[0, t_{1}\right]$, we have $N(t) \leq\left(\frac{\lambda}{\mu}\right)^{\frac{t_{1}}{\tau}+1} W$.

Proof. First we show by induction on $n \geq 0$ that, in the absence of culls:

$$
N(t) \leq\left(\frac{\lambda}{\mu}\right)^{n+1} W \text { for } t \in[n \tau,(n+1) \tau] .
$$

Basis step $(n=0)$. Using lemma 3.12, with $A=0, B=\tau, \xi=W$, and $U=W$, we have:

$$
N(t) \leq \frac{\lambda}{\mu} W+W\left(1-\frac{\lambda}{\mu}\right) e^{-\mu t} \text { for } t \in[0, \tau] .
$$

Since $\lambda \geq \mu$ by assumption, it follows at once that $N(t) \leq \frac{\lambda}{\mu} W$ for $t \in[0, \tau]$, which is the result required on the basis step.

Inductive step. The inductive step is established by the same method as used in the basis step.

Hence (3.82) is established by induction.

To complete the proof, observe that $j \tau<t_{1} \leq(j+1) \tau$ for some $j \geq 0$. Also, by the induction just performed and the fact that $N\left(t_{1}\right)=b_{1} N\left(t_{1}^{-}\right) \leq N\left(t_{1}^{-}\right)$, we have:

$$
N(t-\tau) \leq\left(\frac{\lambda}{\mu}\right)^{j} W \text { for } t \in\left[j \tau, t_{1}\right]
$$

Then, using lemma 3.12 with $A=j \tau, B=t_{1}, \xi=\left(\frac{\lambda}{\mu}\right)^{j} W$, and $U=\left(\frac{\lambda}{\mu}\right)^{j} W$, we obtain, for $t \in\left[j \tau, t_{1}\right]$ :

$$
\begin{aligned}
N(t) & \leq\left(\frac{\lambda}{\mu}\right)^{j+1} W+\left(\frac{\lambda}{\mu}\right)^{j} W\left(1-\frac{\lambda}{\mu}\right) e^{-\mu(t-j \tau)} \\
& \leq\left(\frac{\lambda}{\mu}\right)^{j+1} W \text { since } \frac{\lambda}{\mu} \geq 1 \text { by assumption. }
\end{aligned}
$$

By (3.84) and (3.85) and the assumption that $\frac{\lambda}{\mu} \geq 1$, then $N(t) \leq\left(\frac{\lambda}{\mu}\right)^{j+1} W$ for $t \in$ $\left[0, t_{1}\right]$.

In particular, then:

$$
N(t) \leq\left(\frac{\lambda}{\mu}\right)^{j+1} W \leq\left(\frac{\lambda}{\mu}\right)^{\frac{t_{1}}{\tau}+1} W \text { for } t \in\left[0, t_{1}\right],
$$

since $\frac{t_{1}}{\tau}+1>j+1$ and $\lambda \geq \mu$. 
Theorem 3.14. Suppose $N(t)$ satisfies (3.71) and (3.72) and that $N(t) \leq W$ for $t \in[-\tau, 0]$ where $W$ is a positive constant. Assume $\lambda \geq \mu$. Let the culling regime be such that $\Delta<1$ where $\Delta$ is given by (3.74). Let $\psi=\left(\frac{\lambda}{\mu}\right)^{\frac{t_{1}}{\tau}+1} W$. Then:

$$
N(t) \leq \psi \Delta^{n} \text { on }\left[t_{i_{n}}, t_{i_{n+1}}\right] \text { for } n \geq 1,
$$

where the subsequence $t_{i_{n}}$ is described in definition 3.4 .

Proof. We use induction on $n \geq 1$. Within the basis step and inductive step of this induction, we will perform smaller inductions. Therefore we shall refer to the induction on $n$ as the outer induction and to the others as inner inductions.

Basis step (outer induction) $(n=1)$. We need

$$
N(t) \leq \psi \Delta \text { for } t \in\left[t_{i_{1}}, t_{i_{2}}\right]=\left[t_{1}, t_{i_{2}}\right]
$$

Split the interval $\left[t_{1}, t_{i_{2}}\right]$ into the subintervals

$$
\left[t_{1}, t_{2}\right],\left[t_{2}, t_{3}\right], \ldots,\left[t_{i_{2}-1}, t_{i_{2}}\right]
$$

Since $t_{i+1}-t_{i} \leq \tau$ for $i \geq 1$ and $t_{i_{2}} \geq t_{i_{1}}+\tau=t_{1}+\tau$, we know there is at least one subinterval in (3.89).

We can show $N(t) \leq \psi \Delta$ on the subintervals by induction.

Basis step (inner induction). By lemma 3.13 and since $t_{2}-t_{1} \leq \tau$, we know that $N(t-\tau) \leq \psi$ for $t \in\left[t_{1}, t_{2}\right]$. But then we can use lemma 3.12 with $A=t_{1}, B=t_{2}$, $U=\psi, \xi=b_{1} \psi$ to obtain, by equation (3.78),

$$
\begin{aligned}
N(t) & \leq \psi\left[\frac{\lambda}{\mu}+e^{-\mu\left(t-t_{1}\right)}\left(b_{1}-\frac{\lambda}{\mu}\right)\right] \text { for } t \in\left[t_{1}, t_{2}\right] \\
& \leq \psi \Delta \text { by the definition of } \Delta \text { (equation (3.74)) } .
\end{aligned}
$$

Inductive step (inner induction). (This step is needed only if there is more than one subinterval in (3.89), that is, if $i_{2} \geq 3$. Otherwise the basis step suffices.)

Assume (inductive hypothesis) that:

$$
N(t) \leq \psi \Delta \text { for } t \in\left[t_{j}, t_{j+1}\right] \text { for all } j \text { with } 1 \leq j \leq r,
$$

for some $r$, where $1 \leq r \leq i_{2}-2$.

We wish to show that $N(t) \leq \psi \Delta$ for $t \in\left[t_{r+1}, t_{r+2}\right]$.

By the initial data and lemma 3.13, we know that $N(t-\tau) \leq \psi$ for $t \in\left[0, t_{1}\right]$. Also, by the inductive hypothesis (3.91), we know, for $t \in\left[t_{1}, t_{r+1}\right]$, that $N(t-\tau) \leq \psi \Delta \leq \psi$, using the assumption that $\Delta<1$.

Hence $N(t-\tau) \leq \psi$ for $t \in\left[0, t_{r+1}\right]$. 
But then we can again use lemma 3.12, this time with $A=t_{r+1}, B=t_{r+2}, U=\psi$, $\xi=b_{r+1} \psi$ to obtain, by equation (3.78),

$$
\begin{aligned}
N(t) & \leq \psi\left[\frac{\lambda}{\mu}+e^{-\mu\left(t-t_{r+1}\right)}\left(b_{r+1}-\frac{\lambda}{\mu}\right)\right] \text { for } t \in\left[t_{r+1}, t_{r+2}\right] \\
& \leq \psi \Delta \text { by the definition of } \Delta \text { (equation (3.74)). }
\end{aligned}
$$

Hence, by induction, $N(t) \leq \psi \Delta$ for $t \in\left[t_{1}, t_{i_{2}}\right]$.

This completes the basis step of the outer induction.

Inductive step (outer induction) Assume (inductive hypothesis) that:

$$
N(t) \leq \psi \Delta^{r} \text { for } t \in\left[t_{i_{r}}, t_{i_{r+1}}\right] \text { for some } r \geq 1 .
$$

Then we wish to show that

$$
N(t) \leq \psi \Delta^{r+1} \text { for } t \in\left[t_{i_{r+1}}, t_{i_{r+2}}\right] .
$$

Split the interval $\left[t_{i_{r+1}}, t_{i_{r+2}}\right]$ into the following subintervals:

$$
\left[t_{i_{r+1}}, t_{i_{r+1}+1}\right],\left[t_{i_{r+1}+1}, t_{i_{r+1}+2}\right], \ldots,\left[t_{i_{r+2}-1}, t_{i_{r+2}}\right] .
$$

There is at least one subinterval from the way the way the $t_{i_{r}}$ are defined (definition 3.4) and the assumption that $t_{i+1}-t_{i} \leq \tau$ for $i \geq 1$.

We establish (3.94) by induction on the subintervals (3.95) using (3.93). This will be similar to the basis step (outer induction) which we just completed.

Thus we have completed the outer induction and established (3.87).

Corollary 3.1. Make the assumptions in theorem 3.14. Then $N(t) \rightarrow 0$ as $t \rightarrow \infty$.

Proof. The result follows immediately from equation (3.87) in theorem 3.14, the assumption that $\Delta<1$, and the fact that $t_{i_{n}} \rightarrow \infty$ as $n \rightarrow \infty$.

Remark 1. It is all very well to show that the culling regime succeeds when $\Delta<1$, but is this actually possible? In subsection 3.4 .4 below, we will see that for a sufficiently strong regime, we can always ensure $\Delta<1$.

Remark 2. Notice that the culling regime not only succeeds in ensuring $N(t) \rightarrow 0$ as $t \rightarrow \infty$, but it also does so in such a way that $N(t)$ can be bounded above by a decreasing sequence, so we can be confident that the adult pest population will not flare up significantly (and thereby potentially ruin crops or cause an epidemic) before dying out. Notice further that theorem 3.14 trivially allows us to find an upper bound for the time it will take to reduce the pest population to be below a particular level. Such an upper bound is potentially extremely useful. After all, given that prolonged pesticide programs could lead to pesticide resistance and other problems (see section 2.3) and could also be quite expensive, it may be decided in many situations to apply pesticides only until a pest population has been suitably reduced. 
Remark 3. If we replace the linear model (3.72) by the differential inequality

$$
\frac{d N(t)}{d t} \leq \lambda N(t-\tau)-\mu N(t) \text { for } t \geq 0, t \neq t_{i} \text { where } i \geq 1,
$$

then the methods of this subsection can again be applied to prove that the culling regime will be successful provided $\Delta<1$. Hence there will exist a successful culling regime even when $\lambda<\mu$, since we will have

$$
\frac{d N(t)}{d t} \leq \mu N(t-\tau)-\mu N(t) \text { for } t \geq 0, t \neq t_{i} \text { where } i \geq 1,
$$

so that the culling regime with $\Delta<1$ and in which $\lambda$ is set to equal $\mu$ will succeed. Of course if $\lambda<\mu$ the population will die out naturally, so culling is unnecessary.

Remark 4. Suppose the restriction that $t_{i+1}-t_{i} \leq \tau$ for $i \geq 1$ is weakened to $t_{i+1}-t_{i} \leq k \tau$ for some fixed finite natural number $k>1$. Then our intuition (gained from calculations which we do not include out of considerations of space) leads us to believe that methods not dissimilar in principle to those used in this subsection, but potentially very dissimilar in terms of tractability, could be employed to construct a successful culling regime.

\subsubsection{Nicholson model}

In the Nicholson model (equation (3.27)), we know by theorem 3.4 that when $\lambda_{1}<$ $\mu$, then $N(t) \rightarrow 0$ as $t \rightarrow \infty$ in such a way that $N(t)$ can be bounded above by a decreasing sequence.

However if $\lambda_{1}>\mu$ then by theorems 3.5 to 3.9 the pest will persist, given certain additional conditions on the model parameters or initial conditions. The weight of evidence in the literature would seem to suggest that if $\lambda_{1}>\mu$ then the pest will never naturally go extinct (we will not have $N(t) \rightarrow 0$ as $t \rightarrow \infty$ ). Therefore culling is warranted in this case if the pest is causing serious harm.

Now it is clear that $\lambda_{1} N e^{-\lambda_{2} N}<\lambda_{1} N$ for $N>0$. But we have said (paragraph 2, section 3.4) that introducing impulsive culling into our models preserves positivity. Hence for the Nicholson model (equation (3.27)) we can write, for $t \geq 0$, that:

$$
\frac{d N(t)}{d t}=\lambda_{1} N(t-\tau) e^{-\lambda_{2} N(t-\tau)}-\mu N(t) \leq \lambda_{1} N(t-\tau)-\mu N(t) .
$$

But then by remark 3 at the end of subsection 3.4.1, we see that the culling regime of subsection 3.4 .1 for the linear model, with $\lambda$ replaced by $\lambda_{1}$, will successfully eradicate the adult population $N(t)$ if $\Delta<1$. Thus, to be precise, a culling regime for the Nicholson model will succeed if:

$$
\Delta_{2}=\sup _{i \geq 1}\left(\frac{\lambda_{1}}{\mu}+e^{-\mu\left(t_{i+1}-t_{i}\right)}\left(b_{i}-\frac{\lambda_{1}}{\mu}\right)\right)<1 .
$$

For the Nicholson model, with a culling regime satisfying (3.99), remark 2 at the end of subsection 3.4.1 still applies. The regime will eradicate the pest in such a way that $N(t)$ can be bounded above by a decreasing sequence. 


\subsubsection{Allee model}

In the Allee model (equation (3.33)), we know by theorem 3.10 that when $\alpha_{1}<e \alpha_{2} \mu$, then $N(t) \rightarrow 0$ as $t \rightarrow \infty$ in such a way that $N(t)$ can be bounded above by a decreasing sequence.

However if $\alpha_{1}>e \alpha_{2} \mu$ then by theorem 3.12 the pest will persist, given certain additional conditions on the model parameters and initial data. Simulations we have performed (but chosen not to include because it takes less space to describe them in words) suggest to us that if $\alpha_{1} \geq e \alpha_{2} \mu$ then the pest will never naturally go extinct (we will not have $N(t) \rightarrow 0$ as $t \rightarrow \infty$ ) provided that $N(t)>N_{1}$ for $t \in[-\tau, 0]$ (where $N_{1}$ is defined as in results 2) and 3) of lemma 3.5). Therefore culling may be warranted in this case if the pest is causing serious harm.

Now it is trivial to show that $\alpha_{1} N^{2} e^{-\alpha_{2} N} \leq\left(\frac{\alpha_{1}}{\alpha_{2} e}\right) N$ for $N>0$. But we have said (paragraph 2, section 3.4) that introducing impulsive culling into our models preserves positivity. Hence for the Allee model (equation (3.33)) we can write, for $t \geq 0$, that:

$$
\frac{d N(t)}{d t}=\alpha_{1}(N(t-\tau))^{2} e^{-\alpha_{2} N(t-\tau)}-\mu N(t) \leq\left(\frac{\alpha_{1}}{\alpha_{2} e}\right) N(t-\tau)-\mu N(t) .
$$

But then by remark 3 at the end of subsection 3.4.1, we see that the culling regime of subsection 3.4.1 for the linear model, with $\lambda$ replaced by $\frac{\alpha_{1}}{\alpha_{2} e}$, will successfully eradicate the adult population $N(t)$ if $\Delta<1$. Hence, to phrase it more specifically, a culling regime will succeed for the Allee model if:

$$
\Delta_{3}=\sup _{i \geq 1}\left(\frac{\alpha_{1}}{\alpha_{2} e \mu}+e^{-\mu\left(t_{i+1}-t_{i}\right)}\left(b_{i}-\frac{\alpha_{1}}{\alpha_{2} e \mu}\right)\right)<1 .
$$

With a culling regime satisfying (3.101) imposed on the Allee model, remark 2 at the end of subsection 3.4.1 again applies. Eradication will occur in such a way that $N(t)$ can be bounded above by a decreasing sequence. In fact the method of theorem 3.14 will allow us to find, for any $R_{0}>0$, an upper bound for the minimum number of culls needed to ensure $N(t)<R_{0}$ for a time interval of width at least $\tau$ time units. But if $\alpha_{1} \geq e \alpha_{2} \mu$, we can define $N_{1}$ as in results 2) and 3) of lemma 3.5, and then if $N(t)<R_{0}<N_{1}$ for a time interval of width at least $\tau$ time units, then by theorem 3.13, extinction becomes inevitable, even if we stop culling. Hence only a finite number of culls will be needed to guarantee extinction in the Allee model, and we can find an upper bound for the minimum number of culls needed to guarantee extinction.

Let us find such an upper bound explicitly. Recall $b$ and $T$ from equations (3.75) and (3.76) and define

$$
g(T, b)=\frac{\alpha_{1}}{\alpha_{2} e \mu}+e^{-\mu T}\left(b-\frac{\alpha_{1}}{\alpha_{2} e \mu}\right) .
$$

Since culling is not needed when $\alpha_{1}<e \alpha_{2} \mu$, then assume $\alpha_{1} \geq e \alpha_{2} \mu$. Notice that $\Delta_{3} \leq g(T, b)$ since we are assuming $\alpha_{1} \geq e \alpha_{2} \mu$. Suppose $b<1$. Then, since $g(T, b)$ is a continuous function of $T$ and $g(0, b)=b<\frac{b+1}{2}<1$, we know that $g(T, b) \leq \frac{b+1}{2}<1$ for $T$ small enough. Let $T$ be small enough to satisfy this latter inequality and also suppose $t_{i+1}-t_{i}=T \leq \tau$ for all $i \geq 1$. Therefore $\Delta_{3} \leq g(T, b) \leq \frac{b+1}{2}<1$. Let $\theta=\frac{\alpha_{1}}{\alpha_{2} e}$. Then $\theta \geq \mu$. We have the initial data $N(t) \leq W$ for $t \in[-\tau, 0]$ where $W$ is a positive constant. 
If $W<N_{1}$ (where $N_{1}$ is defined as in results 2) and 3) of lemma 3.5) then by theorem 3.13, extinction will occur naturally, and without $N(t)$ flaring up before dying down, so culling is unnecessary. Therefore assume $W>N_{1}$. Bearing in mind remark 3 at the end of subsection 3.4.1, then the method of lemma 3.13 is trivially adapted to the current problem to show that we will have $N(t) \leq \psi=\left(\frac{\theta}{\mu}\right)^{\frac{t_{1}}{\tau}+1} W$ for $t \in\left[0, t_{1}\right]$. Since by assumption $\theta \geq \mu$ and $W>N_{1}$, then $\psi>N_{1}$.

Similarly (bearing in mind remark 3 at the end of subsection 3.4.1 as well as equation (3.100)) we can trivially adapt theorem 3.14 to the current problem to show that $N(t) \leq \psi \Delta_{3}^{n} \leq \psi\left(\frac{b+1}{2}\right)^{n}$ for $t \in\left[t_{i_{n}}, t_{i_{n+1}}\right]$ for $n \geq 1$. Now if $\psi\left(\frac{b+1}{2}\right)^{n}<N_{1}$ then we find that $n>\frac{\ln \left(\frac{\psi}{N_{1}}\right)}{\ln \left(\frac{2}{b+1}\right)}$. So if

$$
n=1+\left\lfloor\frac{\ln \left(\frac{\psi}{N_{1}}\right)}{\ln \left(\frac{2}{b+1}\right)}\right\rfloor,
$$

where $U$ denotes the greatest integer function, then certainly $N(t)<N_{1}$ for $t \in$ $\left[t_{i_{n}}, t_{i_{n+1}}\right]$, by which we may conclude, using theorem 3.13 and the fact that $t_{i_{n+1}}-$ $t_{i_{n}} \geq \tau$ by definition 3.4 , that extinction will now be inevitable without further culling. Hence a finite upper bound for the minimum number of culls needed to guarantee extinction is given by (3.102).

The upper bound (3.102) is valuable for several reasons. Firstly, insects adapt quickly to pesticides, so if we only need a finite number of culls to eradicate the pest, then eradication may occur before the pest develops resistance to the pesticide. Secondly, an upper bound for the number of culls needed for eradication allows a farmer to estimate how much pesticide to buy or a manufacturing plant to estimate how much pesticide to manufacture.

Unfortunately, however, the concept of eradication in only finitely many culls may apply as much to non-target species, including pollinators such as bees, as it does to pests. Indeed we may inadvertently have uncovered one of the causes of Colony Collapse Disorder in which bee colonies suddenly collapse [39] - pesticides may help to reduce the population of a hive until it is no longer viable and collapses [53]. There has been a worrying increase in the number of colonies collapsing in recent years [39]. A proper discussion of pesticides is given in section 2.3.

\subsubsection{Regime existence}

We have seen in theorem 3.14 that, for the linear model, the culling regime of definition 3.3 will succeed if $\Delta<1$ where $\Delta$ is given by (3.74). Notice that if $\lambda>\mu$ then $\Delta<1$ cannot hold unless $b_{i}<1$ for all $i \geq 1$, so every cull must have at least some effect, however small. The condition that $\Delta<1$ will certainly hold if:

$$
\Delta \leq f(T, b)=\frac{\lambda}{\mu}+e^{-\mu T}\left(b-\frac{\lambda}{\mu}\right)<1,
$$

where $T=\sup _{i \geq 1}\left(t_{i+1}-t_{i}\right) \leq \tau$ and $b=\sup _{i \geq 1} b_{i}$. But for any $b$ satisfying $0<b<1$, it is clear that (3.103) will hold for $T>0$ if $T$ is sufficiently small. After all, inequality (3.103) holds when $T=0$ and $f(T, b)$ is a continuous function of $T$. Thus, there exists a successful culling regime no matter how weak the culls are, provided they each do something, and as long as the culls are performed frequently enough. This is 

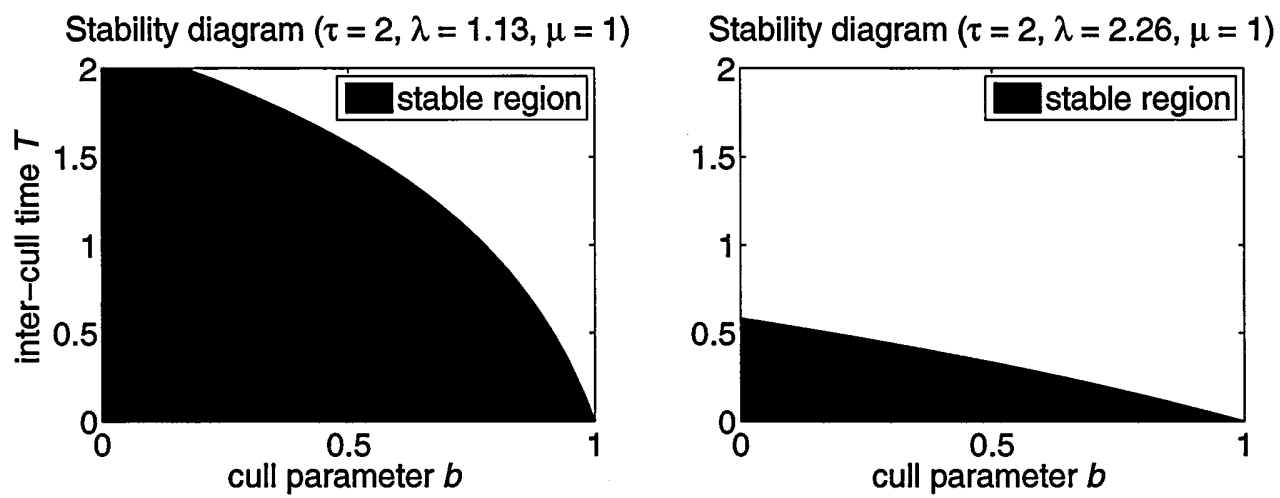

Figure 3.2: Stability diagrams for culling regime. See subsection 3.4.4 for a description.

useful from an agricultural point of view, for example, because a farmer will not have control over how strong pesticides are maufactured to be but he will presumably have some control over how often he can apply the pesticide.

To gain insight into how restrictive condition (3.103) is, we plot "stability" diagrams in figure 3.2. The shaded region in each plot indicates the part of the $T-b$ parameter space for which the culling regime will be guaranteed to succeed by theorem 3.14. The boundaries of the stable region in each plot are $0<b<1,0<T \leq \tau$, and $f(T, b)=1$. We have performed simulations indicating that culling regimes lying outside the stable region may also succeed (the simulations are not included, for the sake of brevity) and a useful research question would be to find all successful regimes. Notice in figure 3.2 how radically the stable region is reduced by doubling the birth rate parameter $\lambda$. This is intuitively sensible because if the pest reproduces faster, we would expect to have to implement a stronger culling regime to control it.

Our comments on regime existence for the linear model are equally relevant to the Nicholson and Allee models. After all, our condition ensuring a regime will succeed in the linear model (namely $\Delta<1$ where $\Delta$ is given by (3.74)) is qualitatively the same as our condition for success in the Nicholson model (namely $\Delta_{2}<1$ where $\Delta_{2}$ is given by (3.99)) and our condition for success in the Allee model (namely $\Delta_{3}<1$ where $\Delta_{3}$ is given by (3.101)).

\subsection{Simulations}

To conduct impulsive culling simulations, we make some simple assumptions. In any particular simulation let all culls have the same strength $b$ (b is not the same for all simulations). Then let the inter-cull time be a constant $T \leq \tau$. By equation (3.103), a culling regime will succeed in the linear model if

$$
\frac{\lambda}{\mu}+e^{-\mu T}\left(b-\frac{\lambda}{\mu}\right)<1 .
$$


In our linear model simulation, we first choose $b$ and then we find $T$ by setting equality in (3.104) and rearranging to find $T$ in terms of $b$ :

$$
T=\frac{1}{\mu} \ln \left(\frac{\lambda-b \mu}{\lambda-\mu}\right)
$$

Of course changing an inequality to equality will mean that the regime is not strictly guaranteed to succeed, but for our simulation it certainly seems to.

In our Nicholson model simulation we follow the same approach. Thus, by condition (3.99), a culling regime will succeed if

$$
\frac{\lambda_{1}}{\mu}+e^{-\mu T}\left(b-\frac{\lambda_{1}}{\mu}\right)<1 .
$$

For a given $b$ we then choose $T$ by setting equality in (3.106) and rearranging to find $T$.

Finally we adopt the same approach for the Allee model. By condition (3.101), a culling regime will succeed if

$$
\frac{\alpha_{1}}{\alpha_{2} e \mu}+e^{-\mu T}\left(b-\frac{\alpha_{1}}{\alpha_{2} e \mu}\right)<1 .
$$

Again, for a given $b$, we then choose $T$ by setting equality in (3.107) and rearranging to find $T$.

In all simulations we set $\tau=1$ and the initial data is $N(t)=1$ for $t \in[-\tau, 0]$. Figure 3.3 shows simulations of the linear, Nicholson, and Allee models, with culling regimes imposed on them as just described. In the Allee model simulation, we stop culling when $N(t)$ has been kept below $N_{1}$ for at least $\tau$ consecutive time units, so that natural extinction will then occur (see theorem 3.13).

The figure also shows (bottom right) for the Nicholson model that when culls do not occur as often as required by our results, then the average adult pest population can be maintained above the level it would remain at naturally. Both culling and "natural" simulations are the same until the first cull occurs, whereafter the "natural" simulation (dot-dashed line) immediately approaches a positive constant and the simulation with culling (solid line) experiences large and apparently stable oscillations. The average pest population over the inter-cull period in the culling simulation is higher than the constant to which the "natural" simulation tends, so infrequent culling exacerbates the seriousness of the pest. The potential harm of infrequent culling has been observed for a more complicated pest control model by Gourley, Liu, and $\mathrm{Wu}$ [81]. Theoretically analogous is the idea that infrequent pulses in a pulse vaccination strategy can sustain disease levels above their natural levels, a phenomenon encountered by Choisy et al [61]. An analytical exploration of the potential benefit to a pest of culling infrequently is carried out in the next chapter.

\subsection{Discussion}

In this chapter we have formulated three population models for a pest with a life cycle of two stages, namely a juvenile stage and an adult stage. The birth function was different for each model - we considered linear, Nicholson, and Allee birth functions. Since it is unnecessary to apply pesticides if a pest population is crashing naturally to 

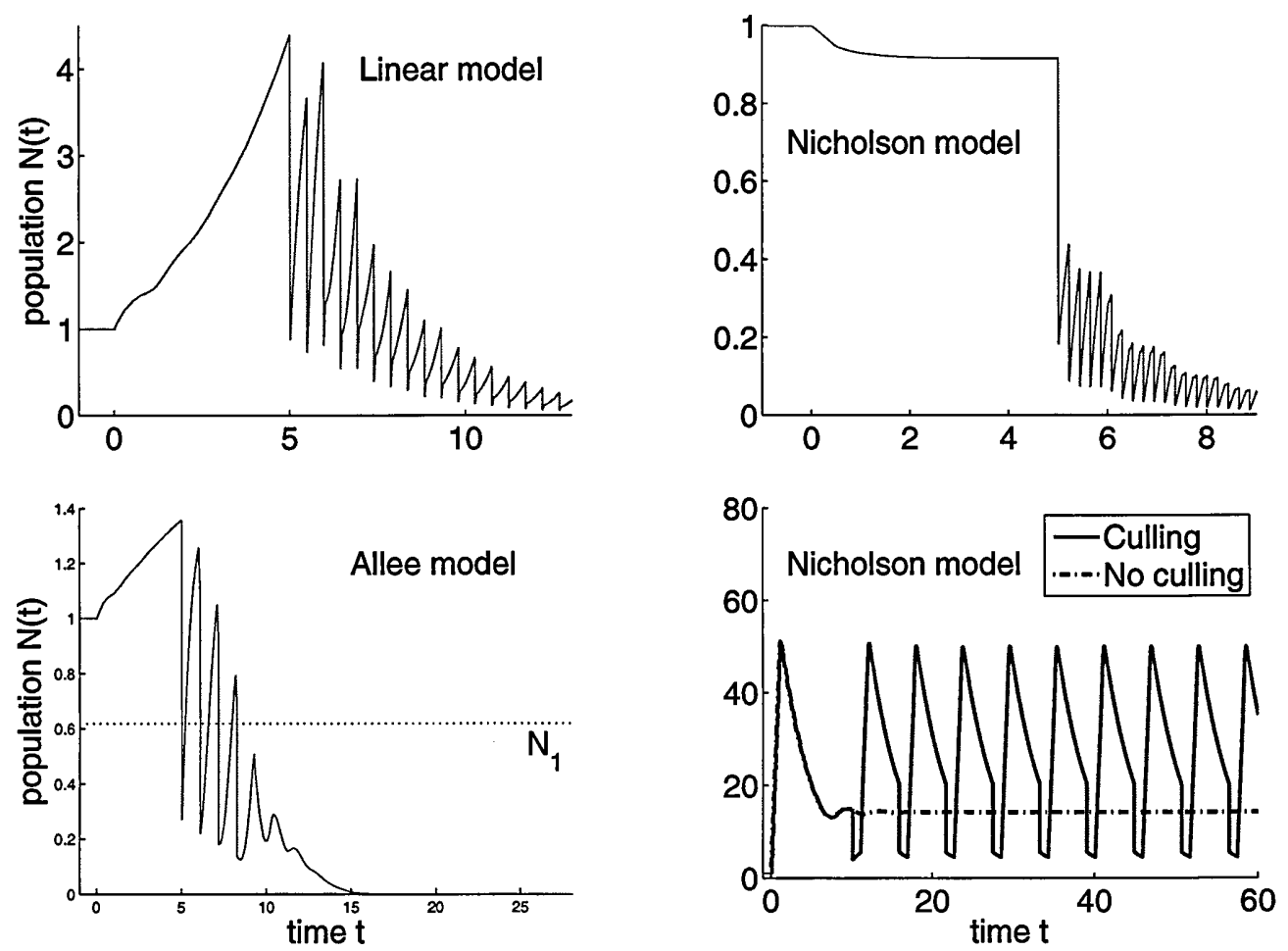

Figure 3.3: Culling simulations. Top left: $\lambda=3, \mu=2, t_{1}=5, b=0.2, T=0.4778$. Top right: $\lambda_{1}=5, \lambda_{2}=1, \mu=2, t_{1}=5, b=0.2, T=0.2137$. Bottom left: $\alpha_{1}=6$, $\alpha_{2}=1, \mu=2, t_{1}=5, b=0.2, T=1$. Bottom right: $\lambda_{1}=75, \lambda_{2}=0.4, \mu=0.25$, $t_{1}=10, b=0.28, T=5.8$. See section 3.5 for descriptions of these plots. 
extinction, we first found natural extinction and endemicity results and only then constructed successful adult impulsive culling regimes for implementation in the event of natural endemicity.

Of particular significance was the calculation of an explicit finite upper bound for the number of culls needed to guarantee extinction in the Allee model. But a simulation for the Nicholson model revealed as well that infrequent culling can make a pest more abundant than in the total absence of culling. In the next chapter we seek analytical confirmation that infrequent culling can, perversely, benefit a pest.

Future research involving the models of the present chapter could involve allowing the culling times to depend on the pest population size. After all, in reality, a farmer may only choose to perform culls when a pest becomes prevalent enough to cause noticeable damage to a crop. 


\title{
Chapter 4
}

\section{Perverse consequences of infrequent culling}

\author{
"Nothing is so unbelievable that oratory cannot make it acceptable."
}

Marcus Tullius Cicero (106 BCE - 43 BCE)

\subsection{Introduction}

We have seen in the previous chapter (section 3.4) that when a pest is culled sufficiently often then it will be eradicated, assuming of course that complications such as pesticide resistance do not arise. We also glimpsed the potential consequences of culling regularly but not sufficiently often to ensure eradication, for in section 3.5 a simulation (figure 3.3, bottom right, which is reproduced below, with minor formatting differences, in figure 4.1) demonstrated, perversely, that infrequent culling can maintain the average population (average over the inter-cull period) at an unnaturally high level.

We can make a generic definition of "infrequent" culling to be where consecutive culls are always further apart than $\tau$ time units where $\tau$ is the maturation age, which in effect means that consecutive culls are further apart than the reproductive cycle, giving the species time (though perhaps not a lot of time) to react and recover from each cull.

Notice that the observation that infrequent culling may benefit a pest is not a new discovery, for in a recent paper by Gourley, Liu, and $\mathrm{Wu}$, it was seen by simulation that infrequent adult impulsive culling of a mosquito vector in a model for West Nile virus can increase the average number of adult mosquitoes (see figure 4 in [81]).

However, no analytic proof that infrequent culling can benefit a pest has been given to date, at least to our knowledge. Therefore in this chapter we shall seek to understand (in section 4.3), in terms of the properties of the birth function, why regular but infrequent culling can benefit a pest. The insight that we gain will be used (in sections 4.4 and 4.5 ) to construct a simple but biologically plausible birth function and 


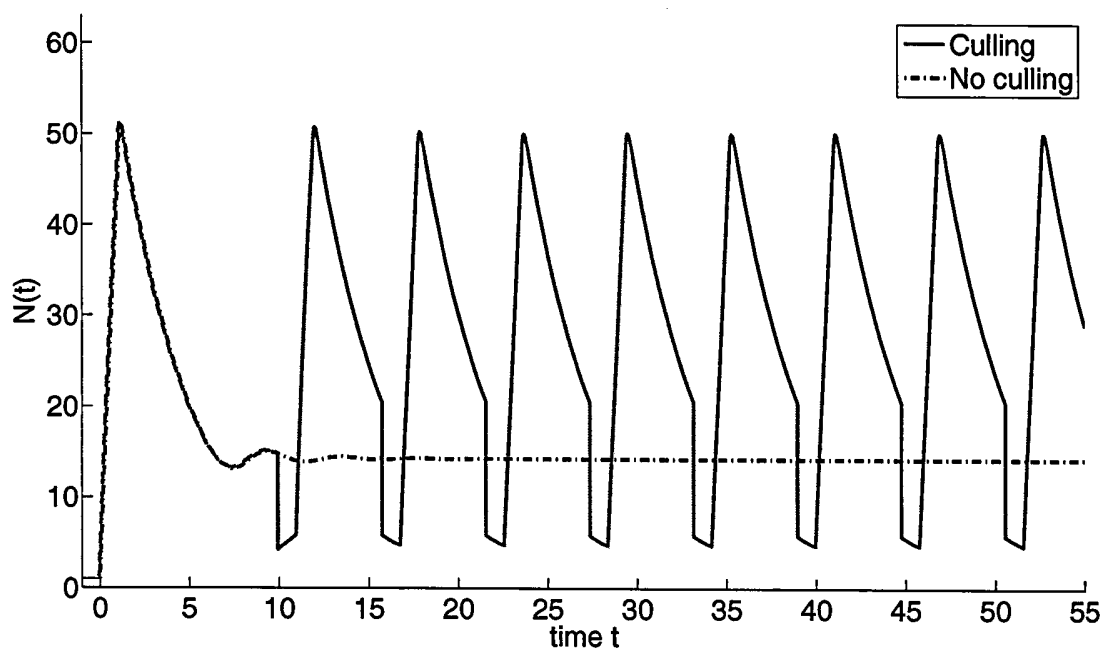

Figure 4.1: Infrequent culling simulation. Here we simulate (4.4) subject to (4.1). The birth function is $b(N)=\lambda_{1} N e^{-\lambda_{2} N}$ where $\lambda_{1}=75 e$ and $\lambda_{2}=0.4$. The initial data is $N(t)=1$ for $t \in[-\tau, 0]$ where $\tau=1$. The juvenile and adult death rates are $\mu_{j}=1$ and $\mu=0.25$ respectively. Culls begin at time $t_{1}=10$ and occur thereafter every $T=5.8$ time units. The cull strength is constant: $b_{i}=0.28$ for $i \geq 1$.

a specific culling regime such that an analytic demonstration of the benefit to the pest becomes straightforward. This analytic demonstration will be given in section 4.6. Simulations in section 4.7 will corroborate our findings and a discussion will end the chapter in section 4.8 .

\subsection{The model}

We remind ourselves of the model of chapter 3 by repeating equations (3.1) and (3.2):

$$
N(t)=\phi(t) \geq 0 \text { for } t \in[-\tau, 0],
$$

and

$$
\frac{d N(t)}{d t}=e^{-\mu_{j} \tau} b(N(t-\tau))-d(N(t)) \text { for } t \geq 0 .
$$

Here $N(t)$ is the number of adults at time $t$ in a stage-structured population with two stages (juvenile and adult), $\phi$ is a function representing initial data, $b$ is the birth function, $d$ is the adult death function, $\mu_{j}$ is the juvenile or immature death rate, and $\tau$ is the maturation age. Suppose we let the adult death function be proportional to the number of adults, so that $d(N(t))=\mu N(t)$ where $\mu$ is the adult per capita death rate. Then (4.2) becomes:

$$
\frac{d N(t)}{d t}=e^{-\mu_{j} \tau} b(N(t-\tau))-\mu N(t) \text { for } t \geq 0
$$


Furthermore if we introduce an adult impulsive culling regime then we can write:

$\frac{d N(t)}{d t}=e^{-\mu_{j} \tau} b(N(t-\tau))-\mu N(t)$ for $t \geq 0, t \neq t_{i} \quad$ and $\quad N\left(t_{i}\right)=b_{i} N\left(t_{i}^{-}\right)$for $i \geq 1$.

Here $t_{i}$ is the time of the $i$-th cull $(i \geq 1)$, which occurs with strength or intensity $b_{i}$, and $t_{i}^{-}$is the time "momentarily" before $t_{i}$. Notice that $b_{i}$ is the proportion of adults left after the $i$-th cull, so for a stronger cull, $b_{i}$ is smaller. Clearly $0 \leq b_{i} \leq 1$. Henceforth we will use the term "population" as a shorthand for the adult population $N(t)$.

In our analysis in chapter 3 , we absorbed the constant $e^{-\mu_{j} \tau}$ into the birth function in equation (3.2). For the purposes of constructing an analytic demonstration that culling can benefit a pest, it will be of value to treat the maturation age $\tau$ as an independent variable. We will still think of the maturation age as being constant but we will give ourselves flexibility in choosing this constant, which will give us more freedom in establishing circumstances such that culling increases an average pest population. Consequently the absorption of the term $e^{-\mu_{j} \tau}$ into the birth function in model (4.3) or model (4.4) will not be a sensible simplification in the analysis of this chapter.

\subsection{The role of the birth function}

We mentioned in section 4.1 the simulation in figure 4.1 showing that infrequent culling can maintain the average pest population at an unnaturally high level. In fact, figure 4.1 shows that, without culling, the pest population tends to a positive constant and that, when subject to the particular culling regime chosen for the simulation, the population exhibits steady oscillations where the period equals the inter-cull time. Moreover the mean value for this periodic solution, taken over one such inter-cull period, significantly exceeds the constant to which the population tends in the absence of culling.

Let us now try to account for this behaviour in terms of the shape of the birth function. The birth function used in figure 4.1 is of Nicholson-type. Recall the description of this type of birth function in subsection 3.2.1 and notice its shape as illustrated in figure 3.1 (middle). A Nicholson birth function $b(N)$ is biggest when the population $N$ is small or intermediate, whereas $b(N)$ is small if $N$ is very small or if $N$ is large.

Consider what happens in the absence of culling if the initial data $N(t)=\phi(t)$ for $t \in[-\tau, 0]$ is in the intermediate range of $N$ for which $b(N)$ is large. One can envisage that the population will grow on the interval $t \in[0, \tau]$, provided the death rate $\mu$ is not too high, because $b(N(t-\tau))$ will be large in equation (4.3). The population, then, will be higher for $t \in[0, \tau]$ than it was initially. For the next time interval $t \in[\tau, 2 \tau]$, the term $b(N(t-\tau))$ may now be smaller, because $N(t-\tau)$ will have grown and $b(N)$ becomes small for large $N$. Suppose $N(t-\tau)$ has become large enough that $b(N(t-\tau))$ is small. Then by equation (4.3) we realise that $N(t)$ will decrease. Indeed it may decrease to be around the same level it was for the initial data. If this is so, then the pattern just described may repeat indefinitely (stable oscillations have been observed in the model (4.3), as mentioned at the end of subsection 3.3.2 and in [82]) or the oscillations may die down as the population tends to a fixed point (see figure 4.1 and theorem 3.7). 
It is the discrepancy in the size of the birth function $b(N)$ for intermediate $N$ and large $N$, as well as the delayed response of the system because (4.3) is a delay differential equation, that permit oscillations to occur at all. In the absence of delay, $N(t)$ would tend monotonically to a fixed point.

Now consider what happens when a culling regime is imposed in the situation where, in the absence of culling, $N(t)$ tends to a fixed point. Imagine as before that the initial data is in the intermediate range of $N$ for which $b(N)$ is large. We have seen how this may cause $b(N)$ to then increase before eventually decreasing again. Suppose we cull the population before it has decreased to be in the intermediate range. We choose a cull strength which reduces $N(t)$ to be a little bigger than the $N$ for which $b(N)$ is maximum. How $N(t)$ then behaves will depend upon $N(t-\tau)$. But recently (if "now" is the time of the cull) the population was above the intermediate range of $N$ for which $b(N)$ is large. Conceivably, then, $N(t-\tau)$ is large enough that $b(N(t-\tau))$ will not be large. Then, by equation (4.3), $N(t)$ may decrease across the range of intermediate $N$. If $N(t)$ has not decreased below the range of intermediate $N$ before $\tau$ time units has elapsed, then we may have recovered the initial data, which was chosen to be in the intermediate range of $N$. The pattern just described may now repeat.

Thus, strategically chosen culling may create a periodic solution when, in the absence of culling, the population would tend to a fixed point. If $b(N)$ is large enough for intermediate $N$, then initial data (for $t \in[-\tau, 0]$ ) in this intermediate range of $N$ will cause $N(t)$ to increase quite significantly when $t \in[0, \tau]$. We envisage a scenario in which $b(N)$ is sufficiently large for intermediate $N$ such that if culling does create a periodic solution then the average value of $N(t)$ over one period is larger than the fixed point to which $N(t)$ tends in the absence of culling. By creating this scenario, we have established a mechanism to explain the features revealed by figure 4.1.

\subsection{A simple birth function}

Motivated by the previous section, we note that a birth function can be large for intermediate $N$ and smaller for larger $N$ if it satisfies:

$$
b(N)= \begin{cases}W & \text { for } N_{1} \leq N<N_{2}, \\ M & \text { for } N_{2} \leq N \leq N_{3},\end{cases}
$$

where $N_{1}, N_{2}, N_{3}, M$, and $W$ are positive constants with $N_{1}\left\langle N_{2}\left\langle N_{3}\right.\right.$ and $\left.W\right\rangle$ $M$. The range $N \in\left[N_{1}, N_{2}\right]$ is to be considered "intermediate" and $N \in\left[N_{2}, N_{3}\right]$ is "larger". The behaviour of the birth function $b(N)$ for $N<N_{1}$ and $N>N_{3}$ will not influence our results, so we will allow $b(N)$ to be any plausible birth function for these ranges of $N$. In particular it is plausible to ask for $b(N)$ to increase monotonically in some way from zero to $W$ for $N \in\left[0, N_{1}\right]$ and for $b(N)$ to decrease monotonically from $M$ to zero for $N \geq N_{3}$

How realistic is it to require a birth function $b(N)$ to satisfy (4.5)? The idea that $b(N)$ is roughly constant for intermediate $N$ is relatively sensible. After all, we can expect $b(N)$ to increase with $N$ for small $N\left(0<N<N_{1}\right)$ until competition for food, space, or a successful mating prevent the birth rate from rising any further. The birth rate may not fall immediately, however, because if the population continues to grow then there will be more individuals who can reproduce even though competition will intensify. These two opposing influences - a growing population and growing competition - may balance each other (thereby yielding a flat birth function across intermediate $N$ ) until the competition and other environmental pressures are sufficiently 

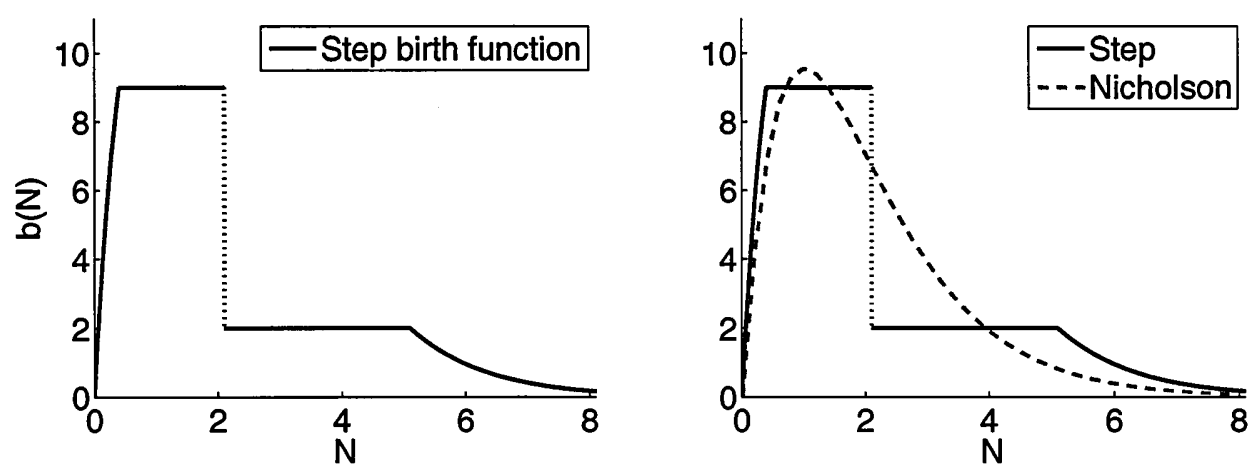

Figure 4.2: Left: a step birth function. Right: Comparison of a step birth function and a Nicholson birth function. For step birth function in both left and right plots: $N_{1}=0.4, N_{2}=2.1, N_{3}=5.1, M=2, W=9, b(N)=\lambda_{1} N e^{-\lambda_{2} N}$ on $\left[0, N_{1}\right]$ where $\lambda_{2}=1$ and $\lambda_{1}=\frac{W}{N_{1}} e^{\lambda_{2} N_{1}}$, and $b(N)=\lambda_{1} N e^{-\lambda_{2} N}$ on $\left[N_{3}, \infty\right)$ where $\lambda_{2}=1$ and $\lambda_{1}=\frac{M}{N_{3}} e^{\lambda_{2} N_{3}}$. Nicholson birth function: $b(N)=26 N e^{-N}$.

serious as to trigger a sudden collapse in the birth rate. After the birth rate $b(N)$ collapses where $N=N_{2}$, say, from some value $W$ to some value $M$, it may remain at roughly $M$ for a range of $N>N_{2}$ because the two opposing forces - a growing population and growing competition or environmental pressures - may once again balance. Otherwise $b(N)$ may decrease to zero for $N>N_{2}$, but if it does this slowly enough at first then $b(N)$ may be approximated by a constant for a range of $N>N_{2}$.

A birth function satisfying (4.5) can be called a step birth function. The appropriateness of such a label is made obvious by plotting such a function, which we do in figure 4.2 (left). In figure 4.2 (right) we compare a step birth function with a Nicholson birth function. The two functions are qualitatively not dissimilar.

\subsection{Model analysis}

The following assumptions will be used in this section, though they will not all be assumed to hold simultaneously:

(A1) The birth function $b(N)$ satisfies (4.5) for $N_{1} \leq N \leq N_{3}$; also $b(N)$ is monotonic increasing for $0<N<N_{1}$ and monotonic decreasing for $N>N_{3}$

(A2) $N(t)$ satisfies $\frac{d N(t)}{d t}=e^{-\mu_{j} \tau} b(N(t-\tau))-\mu N(t)$ for $t \geq 0$

(A3) $N(t)$ satisfies $\frac{d N(t)}{d t}=e^{-\mu_{j} \tau} b(N(t-\tau))-\mu N(t)$ for $t \geq 0$ except at times $t_{i}$ $(i \geq 1)$ where $N(t)$ is impulsively rescaled according to $N\left(t_{i}\right)=b_{i} N\left(t_{i}^{-}\right)$where $t_{i}^{-}$is the time momentarily before $t_{i}$ and $0 \leq b_{i} \leq 1$.

In this section we will show that $N(t)$ can tend to a fixed point $N^{*}$ in the absence of culls and that a culling regime may be chosen which forces $N(t)$ to periodic where the periodic solution will be stated explicitly. 


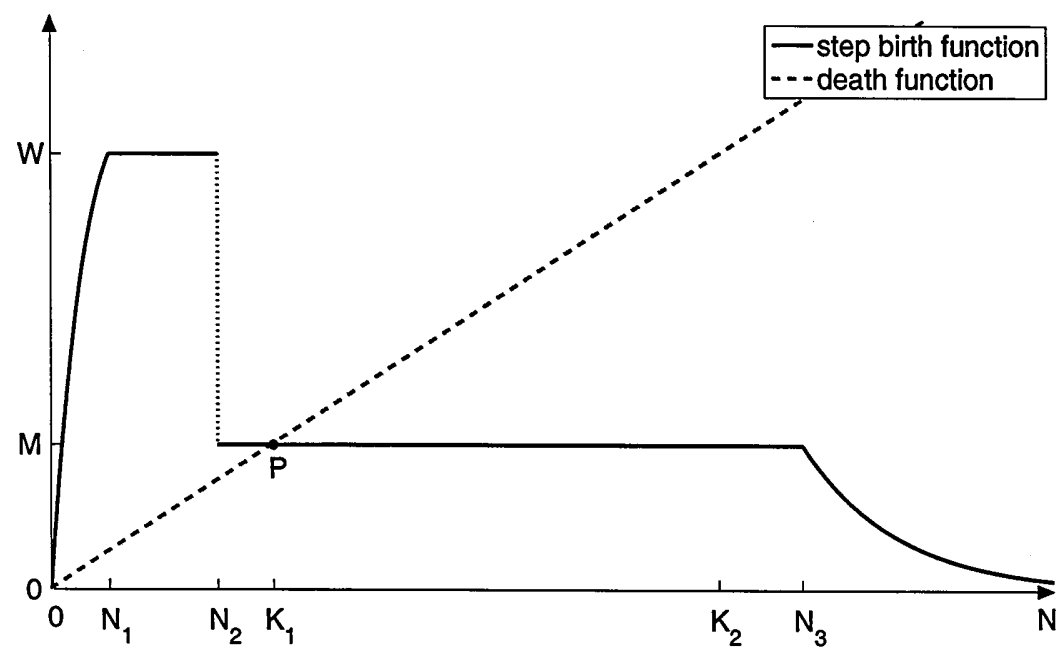

Figure 4.3: Step birth function and death function - picture to accompany theorem 4.1. For step birth function: $N_{1}=0.7, N_{2}=2, N_{3}=9, M=0.8, W=2.4, b(N)=$ $\lambda_{1} N e^{-\lambda_{2} N}$ on $\left[0, N_{1}\right]$ where $\lambda_{2}=1$ and $\lambda_{1}=\frac{W}{N_{1}} e^{\lambda_{2} N_{1}}$, and $b(N)=\lambda_{1} N e^{-\lambda_{2} N}$ on $\left[N_{3}, \infty\right)$ where $\lambda_{2}=1$ and $\lambda_{1}=\frac{M}{N_{3}} e^{\lambda_{2} N_{3}}$. Death function is $\mu N$ where $\mu=0.3$. Here $K_{1}=e^{-\mu_{j} \tau \frac{M}{\mu}}$ and $K_{2}=e^{-\mu_{j} \tau} \frac{W}{\mu}$. The birth and death functions intersect at $\mathbf{P}$, where $N=K_{1}=e^{-\mu_{j} \tau} \frac{M}{\mu}$.

Theorem 4.1. Assume (A1) and (A2) hold. Suppose $\mu$ satisfies $N_{2}<e^{-\mu_{j} \tau} \frac{M}{\mu}<e^{-\mu_{j} \tau} \frac{W}{\mu}<$ $N_{3}$. Also suppose $N(t) \in\left[N_{1}, N_{3}\right]$ for $t \in[-\tau, 0]$. Then $N(t) \rightarrow e^{-\mu_{j} \tau} \frac{M}{\mu}=N^{*}$ as $t \rightarrow \infty$.

Proof. Figure 4.3 depicts the situation.

Using the initial data, there will always be two options for $t \in[0, \tau]$ : (i) $\frac{d N(t)}{d t}=e^{-\mu_{j} \tau} W-\mu N(t)$, in which case $N(t)$ moves monotonically towards
$e^{-\mu_{j} \tau} \frac{W}{\mu}$

(ii) $\frac{d N(t)}{d t}=e^{-\mu_{j} \tau} M-\mu N(t)$, in which case $N(t)$ moves monotonically towards $e^{-\mu_{j} \tau} \frac{M}{\mu}$.

Since $N_{2}<e^{-\mu_{j} \tau} \frac{M}{\mu}<e^{-\mu_{j} \tau} \frac{W}{\mu}<N_{3}$, it follows that $N(t) \in\left[N_{1}, N_{3}\right]$ for $t \in[0, \tau]$. It then follows by a method of steps induction that $N(t) \in\left[N_{1}, N_{3}\right]$ for all $t>0$. Hence for any time $t>0$, either option (i) or option (ii) will hold. Since $N_{2}<e^{-\mu_{j} \tau} \frac{M}{\mu}<$ $e^{-\mu_{j} \tau \frac{W}{\mu}}<N_{3}$, we must then have $N(t)>N_{2}$ for all $t$ large enough, say $t \geq T$. But then, for all $t \geq T+\tau$ we will have $b(N(t-\tau))=M$ and option (ii) will hold. Hence $N(t) \rightarrow e^{-\mu_{j} \tau} \frac{M}{\mu}$ as $t \rightarrow \infty$.

Observe that if the initial data in theorem 4.1 were defined on the interval $t \in$ $[A, A+\tau]$ for any constant $A \geq-\tau$, instead of on $[-\tau, 0]$, then it is trivial to see 
that the result would still hold, that is, we would still have $N(t) \rightarrow e^{-\mu_{j} \tau} \frac{M}{\mu}$ as $t \rightarrow$ $\infty$. We make this remark in view of the way we define the initial data in the next theorem. Another remark on initial data is made directly after the statement of the next theorem.

Theorem 4.2. Assume (A1) and (A3) hold. Also assume:

(B1) $N_{1} \leq N(0)<N_{2}<e^{-\mu_{j} \tau} \frac{M}{\mu}<e^{-\mu_{j} \tau} \frac{W}{\mu}<N_{3}$

(B2) $e^{-\mu_{j} \tau \frac{M}{\mu}}+\left(N(0)-e^{-\mu_{j} \tau} \frac{M}{\mu}\right) e^{-\mu \tau}=N_{2}$.

Then $N(t)$ satisfies the following function on $[0,2 \tau)$, provided no culls occur on this interval:

$$
\begin{aligned}
& N(t)=e^{-\mu_{j} \tau} \frac{M}{\mu}+\left(N(0)-e^{-\mu_{j} \tau} \frac{M}{\mu}\right) e^{-\mu t} \text { for } t \in[0, \tau] \text { (initial data), } \\
& N(t)=e^{-\mu_{j} \tau} \frac{W}{\mu}+\left(N_{2}-e^{-\mu_{j} \tau} \frac{W}{\mu}\right) e^{-\mu(t-\tau)} \text { for } t \in[\tau, 2 \tau) .
\end{aligned}
$$

If culls occur every $2 \tau$ time units, with the first cull occurring at time $t_{1}=2 \tau$, and where culls have strength

$$
\delta=\frac{N(0)}{e^{-\mu_{j} \tau \frac{W}{\mu}}+\left(N_{2}-e^{-\mu_{j} \tau \frac{W}{\mu}}\right) e^{-\mu \tau}},
$$

then (4.6) and (4.7) repeats as a periodic solution for all subsequent time.

We will prove theorem 4.2 after commenting on the initial data. Notice, then, that the initial data in theorem 4.2 is defined on the interval $t \in[0, \tau]$ as opposed to $[-\tau, 0]$, which is the interval on which the initial data is defined in (4.1). It is not necessary to define initial data on $[-\tau, 0]$. It is simply a convention. We have defined the initial data in theorem 4.2 on the interval $[0, \tau]$ because it makes the notation slightly more elegant in theorem 4.2 and the analysis that follows it. If we were to translate the time variable with the transformation $t \rightarrow t-\tau$, then the initial data in theorem 4.2 would be defined on the interval $t \in[-\tau, 0]$, and other details in theorem 4.2 would also be slightly different. But the method of proof would be essentially the same.

Proof. First note that continuity of the function in (4.6) and (4.7) is assured by assumption (B2). Notice also that $0<\delta<1$ by using assumption (B2) and the fact that $N(0)<N_{2}<e^{-\mu_{j} \tau} \frac{W}{\mu}$ by assumption (B1). To prove that (4.6) and (4.7) satisfy $\frac{d N(t)}{d t}=e^{-\mu_{j} \tau} b(N(t-\tau))-\mu N(t)$ with $b(N)$ defined by assumption (A1), we use (4.6) as initial data on $[0, \tau]$ and deduce that (4.7) holds.

Now by (4.6) and assumption (B1) we know that $N(0) \geq N_{1}$ and that $N(t)$ is increasing on $[0, \tau]$ since $N(0)-e^{-\mu_{j} \tau} \frac{M}{\mu}<0$. Also by (4.6) and assumption (B2), we know that $N(\tau)=N_{2}$. Thus we have $N_{1} \leq N(t) \leq N_{2}$ for $t \in[0, \tau]$, so that $N_{1} \leq N(t-\tau) \leq N_{2}$ for $t \in[\tau, 2 \tau]$. But then, by assumption (A1), $b(N(t-\tau))=W$ for $t \in[\tau, 2 \tau]$. Therefore, using the knowledge that $\frac{d N(t)}{d t}=e^{-\mu_{j} \tau} b(N(t-\tau))-\mu N(t)$ for $t \in[\tau, 2 \tau)$ by assumption (A3), we can write

$$
N(\tau)=N_{2} \quad \text { and } \quad \frac{d N(t)}{d t}=e^{-\mu_{j} \tau} W-\mu N(t) \text { for } t \in[\tau, 2 \tau) .
$$


Solving (4.9) yields (4.7).

To show that the culling regime stated in the theorem forces (4.6) and (4.7) to be periodic, we need only show that the expression in (4.6), with $t$ replaced by $t-2 \tau$, will hold for $t \in[2 \tau, 3 \tau]$. Then the argument in the first part of the proof will ensure the expression in (4.7), with $t$ replaced by $t-2 \tau$, holds for $t \in[3 \tau, 4 \tau)$, and the periodicity will be guaranteed by induction.

Now we have seen that (4.7) holds. But then $N(t)$ is increasing on $[\tau, 2 \tau)$ since, by assumption (B1), we have $N_{2}-e^{-\mu_{j} \tau \frac{W}{\mu}}<0$. Hence:

$$
N(\tau)=N_{2} \leq N(t) \leq e^{-\mu_{j} \tau} \frac{W}{\mu}+\left(N_{2}-e^{-\mu_{j} \tau} \frac{W}{\mu}\right) e^{-\mu \tau} \text { for } t \in[\tau, 2 \tau) .
$$

Using assumption (B1), $e^{-\mu_{j} \tau} \frac{W}{\mu}+\left(N_{2}-e^{-\mu_{j} \tau} \frac{W}{\mu}\right) e^{-\mu \tau}<N_{3}$, so by (4.10) and assumption (A1), we can say that $b(N(t-\tau))=M$ for $t \in[2 \tau, 3 \tau)$. Then, using assumption (A3), we have:

$$
\frac{d N(t)}{d t}=e^{-\mu_{j} \tau} M-\mu N(t) \text { for } t \in[2 \tau, 3 \tau)
$$

We also know by (4.7) that $N\left(2 \tau^{-}\right)=e^{-\mu_{j} \tau} \frac{W}{\mu}+\left(N_{2}-e^{-\mu_{j} \tau} \frac{W}{\mu}\right) e^{-\mu \tau}$ where $2 \tau^{-}$is the time "momentarily" before $2 \tau$. But then by (4.8) we can write $N(2 \tau)=\delta N\left(2 \tau^{-}\right)=$ $N(0)$. Hence to find $N(t)$ on $[2 \tau, 3 \tau)$, we solve (4.11) with the initial condition $N(2 \tau)=$ $N(0)$ to obtain the expression in (4.6), with $t$ replaced by $t-2 \tau$, on the interval $[2 \tau, 3 \tau)$. Finally there is no cull at $t=3 \tau$ and this guarantees continuity of $N(t)$ at $t=3 \tau$, so the expression in (4.6), with $t$ replaced by $t-2 \tau$, is obtained on the interval $[2 \tau, 3 \tau]$. The proof is complete.

\subsection{Influence of culling on long-term mean population}

The inter-cull average value of the periodic solution in theorem 4.2 will depend upon $\tau$. In fact, if we denote the inter-cull average by $N^{*}(\tau)$, then:

$$
\begin{aligned}
N^{*}(\tau)= & \left(\frac{1}{2 \tau}\right) \int_{0}^{2 \tau} N(t) d t=\left(\frac{1}{2 \tau}\right)\left\{\int_{0}^{\tau} N(t) d t+\int_{\tau}^{2 \tau} N(t) d t\right\} \\
= & \left(\frac{1}{2}\right) e^{-\mu_{j} \tau}\left(\frac{M}{\mu}+\frac{W}{\mu}\right) \\
& +\left(\frac{1}{2}\right)\left(\frac{1-e^{-\mu \tau}}{\mu \tau}\right)\left(N(0)+N_{2}-e^{-\mu_{j} \tau}\left(\frac{M}{\mu}+\frac{W}{\mu}\right)\right) .
\end{aligned}
$$

Notice that the inter-cull average value of the periodic solution is equal to the longterm average value, namely $\lim _{t \rightarrow \infty}\left(\frac{1}{t}\right) \int_{0}^{t} N(s) d s$. Notice also that $N(0)$ and $N_{2}$ in (4.12) are related by a condition involving $\tau$, namely condition (B2) in theorem 4.2.

Recall by theorem 4.1 that the fixed point $e^{-\mu_{j} \tau} \frac{M}{\mu}$ is globally asymptotically stable in the absence of culling, where "global" is understood to mean the region to which the initial data is restricted in the theorem. Therefore, to gain insight into how culling 
will influence the long-term mean pest population, let us compare the fixed point $e^{-\mu_{j} \tau} \frac{M}{\mu}$ to the average value of the periodic solution in the model with culling, as derived in theorem 4.2, namely $N^{*}(\tau)$ in (4.12). The influence of the culling regime will clearly depend upon the sign of $N^{*}(\tau)-e^{-\mu_{j} \tau \frac{M}{\mu}}$ or, equivalently, on the sign of $e^{\mu_{j} \tau} N^{*}(\tau)-\frac{M}{\mu}$. To be more specific, if we define the function

$$
h(\tau)=e^{\mu_{j} \tau} N^{*}(\tau)-\frac{M}{\mu},
$$

then the culling regime of theorem 4.2 increases the long-term mean population if $h(\tau)>0$ and decreases it if $h(\tau)<0$. Since it is meaningless to discuss the influence of the culling regime of theorem 4.2 unless that theorem can actually hold, our problem is to investigate the behaviour of the function $h(\tau)$ in (4.13) when conditions (B1) and (B2) of theorem 4.2 hold.

We construct a framework in which conditions (B1) and (B2) of theorem 4.2 will hold, and in which a simple investigation of $h(\tau)$ in (4.13) is possible, by first assuming that $N_{2}, N_{3}, \mu, M$, and $W$ are positive constants satisfying:

$$
N_{2}<\frac{M}{\mu}<\frac{W}{\mu}<N_{3}
$$

We also assume that $\mu_{j}$ is a positive constant.

Next define

$$
\tau_{1}=\left(\frac{1}{\mu_{j}}\right) \ln \left(\frac{\left(\frac{M}{\mu}\right)}{N_{2}}\right) .
$$

Note that $\tau_{1}>0$ because $\frac{M}{\mu}>N_{2}$ by (4.14). We may rearrange (4.15) to obtain

$$
N_{2}=e^{-\mu_{j} \tau_{1}}\left(\frac{M}{\mu}\right)
$$

By (4.14) and (4.16), we notice that

$$
N_{2}<e^{-\mu_{j} \tau} \frac{M}{\mu}<e^{-\mu_{j} \tau} \frac{W}{\mu}<N_{3} \quad \text { for } \tau<\tau_{1} .
$$

Now define $N(0)$ to be a function of $\tau$ as follows:

$$
N(0)=N(0)_{\tau}=e^{-\mu_{j} \tau} \frac{M}{\mu}+e^{\mu \tau}\left(N_{2}-e^{-\mu_{j} \tau} \frac{M}{\mu}\right) .
$$

Note that (4.18) is simply a rearrangement of condition (B2) of theorem 4.2 , so by defining $N(0)$ as in (4.18) we trivially allow condition (B2) to hold. The dependence of $N(0)$ on $\tau$ is encapsulated by the notation $N(0)_{\tau}$.

By (4.17) we know that $N_{2}-e^{-\mu_{j} \tau} \frac{M}{\mu}<0$ for $\tau \in\left(0, \tau_{1}\right)$, so it is not obvious if $N(0)_{\tau}>0$ for every value of $\tau$ such that $\tau \in\left(0, \tau_{1}\right)$. However, if we notice that $N(0)_{\tau}$ is a continuous function of $\tau$ and that $N(0)_{0}=N_{2}>0$, then we may deduce that there exists $\bar{\tau}$ satisfying $0<\bar{\tau}<\tau_{1}$ such that $N(0)_{\tau}>0$ for $\tau \in(0, \bar{\tau})$. If we notice also that $N(0)_{\tau_{1}}=e^{-\mu_{j} \tau_{1}} \frac{M}{\mu}>0$ (using (4.16)), then we may further deduce that there exists $\hat{\tau}$ satisfying $\bar{\tau} \leq \hat{\tau}<\tau_{1}$ such that $N(0)_{\tau}>0$ for $\tau \in\left(\hat{\tau}, \tau_{1}\right)$. We can summarise the results of this paragraph as follows:

$$
N(0)_{\tau}>0 \quad \text { for } \tau \in(0, \bar{\tau}) \cup\left(\hat{\tau}, \tau_{1}\right) \text { where } 0<\bar{\tau} \leq \hat{\tau}<\tau_{1} .
$$


Given that $\tau>0$, routine manipulations allow us to deduce, by (4.16) and (4.18), that $N(0)_{\tau}<N_{2}$ if and only if $\tau<\tau_{1}$. We know by (4.19) that $N(0)_{\tau}>0$ for $\tau \in$ $(0, \bar{\tau}) \cup\left(\hat{\tau}, \tau_{1}\right)$. For any particular $\tau$ such that $\tau \in(0, \bar{\tau}) \cup\left(\hat{\tau}, \tau_{1}\right)$, we can define $N_{1}$ to be any positive constant such that $N_{1}<N(0)_{\tau}$. Combining the observations of the last two sentences with (4.17), we find that:

$$
N_{1}<N(0)=N(0)_{\tau}<N_{2}<e^{-\mu_{j} \tau} \frac{M}{\mu}<e^{-\mu_{j} \tau} \frac{W}{\mu}<N_{3} \quad \text { for } \tau \in(0, \bar{\tau}) \cup\left(\hat{\tau}, \tau_{1}\right) .
$$

Hence condition (B1) of theorem 4.2 holds for $\tau \in(0, \bar{\tau}) \cup\left(\hat{\tau}, \tau_{1}\right)$.

Knowing that conditions (B1) and (B2) of theorem 4.2 hold for $\tau \in(0, \bar{\tau}) \cup\left(\hat{\tau}, \tau_{1}\right)$, we can now investigate the behaviour of the function $h(\tau)$ (defined in (4.13)) for $\tau \in$ $\left(0, \tau_{1}\right)$. Firstly, then, notice that we can write out an explicit expression for $h(\tau)$ as follows:

$$
\begin{aligned}
h(\tau) & =e^{\mu_{j} \tau} N^{*}(\tau)-\frac{M}{\mu} \quad(\text { using (4.13)) } \\
& =\left(\frac{W}{\mu}-\frac{M}{\mu}\right)+\left(\frac{1-e^{-\mu \tau}}{\mu \tau}\right)\left[e^{\mu_{j} \tau}\left(N(0)+N_{2}\right)-\left(\frac{M}{\mu}+\frac{W}{\mu}\right)\right] \\
& \quad(\text { using }(4.12)) \\
& =\left(\frac{W}{\mu}-\frac{M}{\mu}\right)+\left(\frac{1-e^{-\mu \tau}}{\mu \tau}\right)\left[e^{\mu \tau}\left(e^{\mu_{j} \tau} N_{2}-\frac{M}{\mu}\right)+e^{\mu_{j} \tau} N_{2}-\frac{W}{\mu}\right]
\end{aligned}
$$

(using (4.18)).

By l'Hôpital's rule we may deduce that $\lim _{\tau \rightarrow 0}\left(\frac{1-e^{-\mu \tau}}{\mu \tau}\right)=1$. Hence we find by (4.21) that $\lim _{\tau \rightarrow 0} h(\tau)=2\left(N_{2}-\frac{M}{\mu}\right)$. But $N_{2}-\frac{M}{\mu}<0$ by (4.14). Therefore:

$$
\lim _{\tau \rightarrow 0} h(\tau)<0 \text {. }
$$

Moreover we may deduce by (4.16) and (4.21) that

$$
h\left(\tau_{1}\right)=\left(\frac{W}{\mu}-\frac{M}{\mu}\right)\left[1-\left(\frac{1-e^{-\mu \tau_{1}}}{\mu \tau_{1}}\right)\right] .
$$

It is trivial to check that $\frac{1-e^{-\mu \tau}}{\mu \tau}<1$ for $\tau>0$. In particular, then, $\frac{1-e^{-\mu \tau_{1}}}{\mu \tau_{1}}<1$. Also $\frac{W}{\mu}-\frac{M}{\mu}>0$ by (4.14). Therefore by (4.23) we have:

$$
h\left(\tau_{1}\right)>0 .
$$

Since $h(\tau)$ is a continuous function of $\tau$, we may deduce by (4.22) that there exists $\tau^{*}$ satisfying $0<\tau^{*} \leq \bar{\tau}$ such that

$$
h(\tau)<0 \quad \text { for } \tau \in\left(0, \tau^{*}\right) .
$$

Again, since $h(\tau)$ is continuous in $\tau$, we deduce by (4.24) that there exists $\tau^{* *}$ satisfying $\hat{\tau} \leq \tau^{* *}<\tau_{1}$ such that

$$
h(\tau)>0 \quad \text { for } \tau \in\left(\tau^{* *}, \tau_{1}\right) .
$$

Using the fact that $\bar{\tau} \leq \hat{\tau}$ by (4.19) and also using inequalities stated in the last few paragraphs, it is clear that $0<\tau^{*} \leq \tau^{* *}<\tau_{1}$. 
We are now in a position to draw some conclusions about the influence of the culling regime of theorem 4.2 on the mean pest population. Recalling from the second paragraph of this section that the mean population is decreased when $h(\tau)<0$, we see by (4.25) that the culling regime decreases the mean population for all $\tau$ sufficiently small. Notice that this is not an obvious result. Although the culling frequency is high when $\tau$ is small (since culls occur every $2 \tau$ time units in theorem 4.2), the cull strengths are also weak when $\tau$ is small (since $\delta \rightarrow 1$ as $\tau \rightarrow 0$ where $\delta$ is defined in (4.8) and where we use the fact that $N(0)=N(0)_{\tau} \rightarrow N_{2}$ as $\tau \rightarrow 0$ where $N(0)$ is defined in (4.18) or condition (B2) of theorem 4.2).

Now recalling from the second paragraph of this section that the mean population is increased when $h(\tau)>0$, we see by (4.26) that there is a finite range of $\tau$ such that the culling regime of theorem 4.2 increases the mean population.

The analysis of this section has shown that the impact of the culling regime of theorem 4.2 on the mean pest population depends upon the size of the delay $\tau$. The mean population is decreased when $\tau$ is suitably small and increased when $\tau$ belongs to a larger - but still finite - range of values. It would clearly be practical if we could explain this dependence on $\tau$ in biological terms but no immediate explanation presents itself to the author. This issue warrants further research, which could begin with an extensive numerical study involving various forms of unimodal birth function.

\subsection{Simulations}

We can illustrate by simulation the points made in the previous section. We first choose $N_{2}, N_{3}, \mu, M$, and $W$ to be positive constants satisfying (4.14), specifically $N_{2}=1.5, M=2, W=10, \mu=1$, and $N_{3}$ can be anything bigger than $\frac{W}{\mu}=10$ and need not be specificied more precisely for the purposes of carrying out our simulations.

As in section 4.6 , we choose $\mu_{j}$ to be a positive constant. We let $\mu_{j}=0.1$. Notice that we have chosen the juvenile per capita death rate $\mu_{j}$ to be less than the adult per capita death rate $\mu$ to reflect the idea that adults may be driven into the open by the instinct to search for a mate, making them more vulnerable to predation. Given our choices for $N_{2}, M, \mu$, and $\mu_{j}$, we find by (4.15) that $\tau_{1}=2.8768$.

For any particular $\tau \in\left(0, \tau_{1}\right)$, we define $N(0)=N(0)_{\tau}$ as in (4.18), so that condition (B2) of theorem 4.2 will automatically hold. Given the parameter choices stated above in this section, we find by simulation that $N(0)_{\tau}>0$ for $\tau \in\left(0, \tau_{1}\right)$ (see figure 4.4). For any particular $\tau \in\left(0, \tau_{1}\right)$, we can therefore define $N_{1}$ to be any positive constant such that $N_{1}<N(0)_{\tau}$. Condition (B1) of theorem 4.2 will now hold. We need not specify $N_{1}$ in more precise terms to perform our simulations.

For $\tau \in\left(0, \tau_{1}\right)$, we define $h(\tau)$ as in (4.21) and we recall (second paragraph, section 4.6) that the sign of $h(\tau)$ determines the long-term influence of the culling regime of theorem 4.2, with the mean pest population increased if $h(\tau)>0$ and decreased if $h(\tau)<0$. A plot of $h(\tau)$ against $\tau$, for $\tau \in\left(0, \tau_{1}\right)$, is given in figure 4.4 where we have used the parameter choices stated earlier in this section in order to create the plot. Notice that this plot confirms the observations of section 4.6 - there exists $\tau^{*}$ such that $0<\tau^{*}<\tau_{1}$ where $h(\tau)<0$ and $N(0)_{\tau}>0$ for $\tau \in\left(0, \tau^{*}\right)$ (see (4.25)), and there exists $\tau^{* *}$ such that $\tau^{*}<\tau^{* *}<\tau_{1}$ where $h(\tau)>0$ and $N(0)_{\tau}>0$ for $\tau \in\left(\tau^{* *}, \tau_{1}\right)$ (see (4.26)). For the particular example shown in figure 4.4 we can choose $\tau^{*}$ and $\tau^{* *}$ to equal the single root $\tau_{r}$ of $h(\tau)=0$, namely $\tau_{r}=0.2560$. 


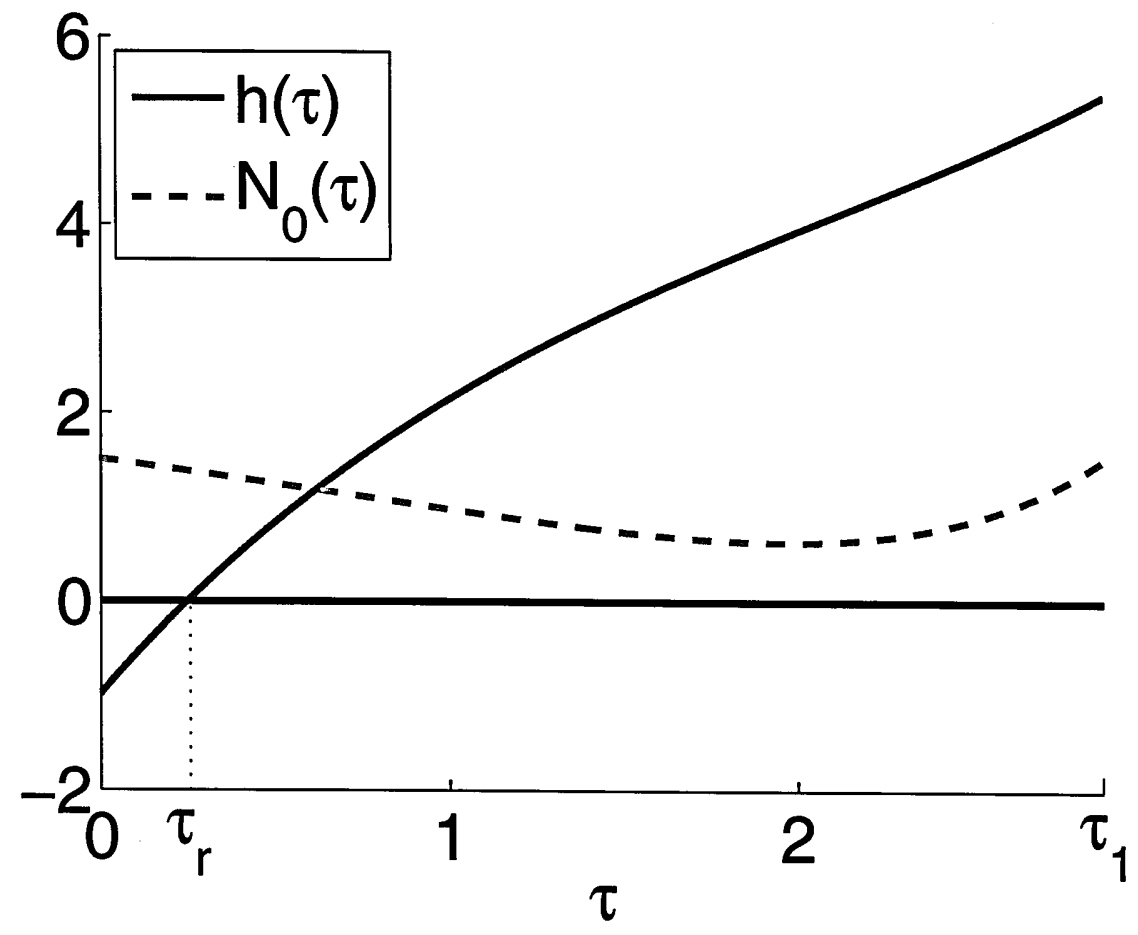

Figure 4.4: A plot of $h(\tau)=e^{\mu_{j} \tau} N^{*}(\tau)-\frac{M}{\mu}$ (see equation (4.21)) and $N_{0}(\tau)$ (see equation (4.18)). As explained in the third and fourth paragraphs of section 4.7 , the conditions of theorem 4.2 hold when $N(0)_{\tau}>0$, and the culling regime of theorem 4.2 increases the mean pest population when $\tau$ is such that $h(\tau)>0$ and decreases it when $\tau$ is such that $h(\tau)<0$. Parameter choice here: $N_{2}=1.5, M=2, W=10$, $\mu=1$, and $\mu_{j}=0.1$. In the plot, $\tau_{1}=2.8768, \tau_{r}=0.2560$, and $h\left(\tau_{r}\right)=0$. 

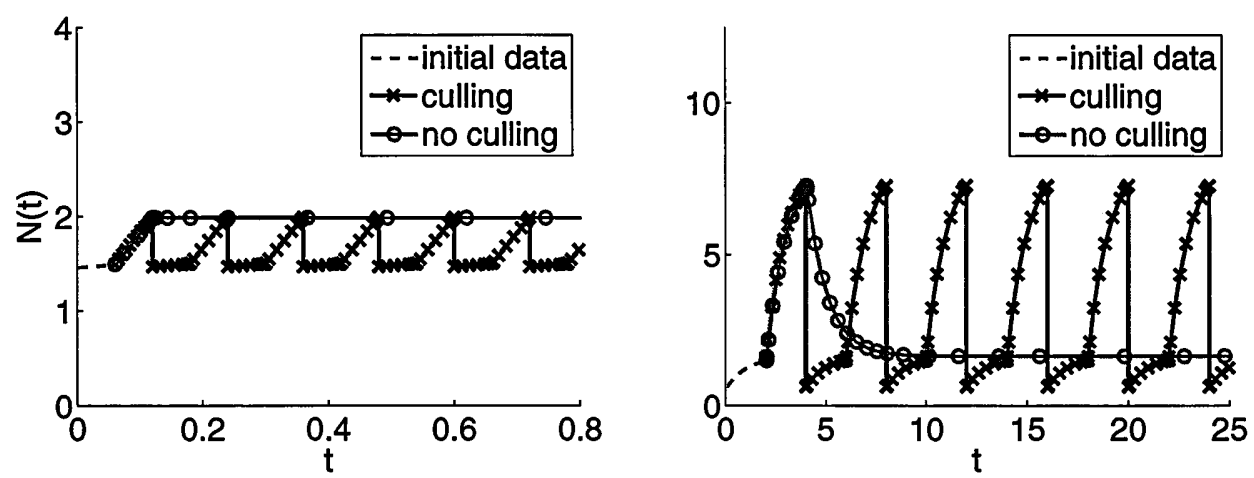

Figure 4.5: Numerical integration of the model for the adult population in the presence of culling (equation (4.4)) and in the absence of culling (equation (4.3)). The birth function is a step birth function, defined as in assumption (A1) at the start of section 4.5, and the initial data is defined as in (4.6). Left: culling decreases the mean. Model parameters: $\mu_{j}=0.1, \mu=1, N_{2}=1.5, M=2, W=10, N_{3}$ can be anything bigger than $\frac{W}{\mu}=10, \tau=0.06, N(0)=e^{-\mu_{j} \tau} \frac{M}{\mu}+e^{\mu \tau}\left(N_{2}-e^{-\mu_{j} \tau} \frac{M}{\mu}\right)=1.4698$, and $N_{1}$ can be anything less than or equal to $N(0)$. Culling regime is as defined in theorem 4.2: the first cull occurs at time $t_{1}=2 \tau=0.12$, culls occur thereafter every $T=2 \tau=0.12$ time units, and all culls have strength $\delta=0.7380$, which is found from equation (4.8). Right: culling increases the mean. Model parameters: Same as left picture except for $\tau=2$ and $N(0)=e^{-\mu_{j} \tau} \frac{M}{\mu}+e^{\mu \tau}\left(N_{2}-e^{-\mu_{j} \tau} \frac{M}{\mu}\right)=0.6218$. Culling regime is as defined in theorem 4.2: the first cull occurs at time $t_{1}=2 \tau=4$, culls occur thereafter every $T=2 \tau=4$ time units, and all culls have strength $\delta=0.0854$, which is found from equation (4.8).

If we make the same parameter choices that are made in figure 4.4 and that are also stated earlier in this section, then we see that the culling regime of theorem 4.2 will decrease the mean population if $\tau \in(0,0.2560)$ and will increase it if $\tau \in(0.2560,2.8768)$. It is obviously worth demonstrating explicitly how the population is influenced by the culling regime, so we do this for two values of $\tau$ in figure 4.5. In the left plot of figure 4.5 we have $\tau=0.06$, so we know that the culling regime will decrease the mean population, and indeed the plot clearly confirms this. In the right plot of figure 4.5 we have $\tau=2$, so we know that the regime will increase the mean population, and this is confirmed by the plot. The two plots further corroborate theorem 4.2 in that they show that the culling regime forces the population $N(t)$ to be periodic with period $2 \tau$.

The right plot of figure 4.5 bears some resemblance to the plot involving a Nicholson birth function in figure 4.1, although there are differences too - the shapes of the peaks in the right plot of figure 4.5 are almost mirror images of the peaks in figure 4.1.

In spite of figure 4.4, it should not be assumed that $N_{0}(\tau)$ is always positive when $\tau \in\left(0, \tau_{1}\right)$ or that $h(\tau)$ is always monotonic increasing for $\tau \in\left(0, \tau_{1}\right)$. Indeed, if we retain the parameter choices of figure 4.4 except for our choice of $N_{2}$, which we reduce from 1.5 to 1 , then $N_{0}(\tau)$ becomes negative on the interval $0<\tau<\tau_{1}$ and $h(\tau)$ becomes decidedly non-monotonic, as we see in figure 4.6. When we bear in mind 


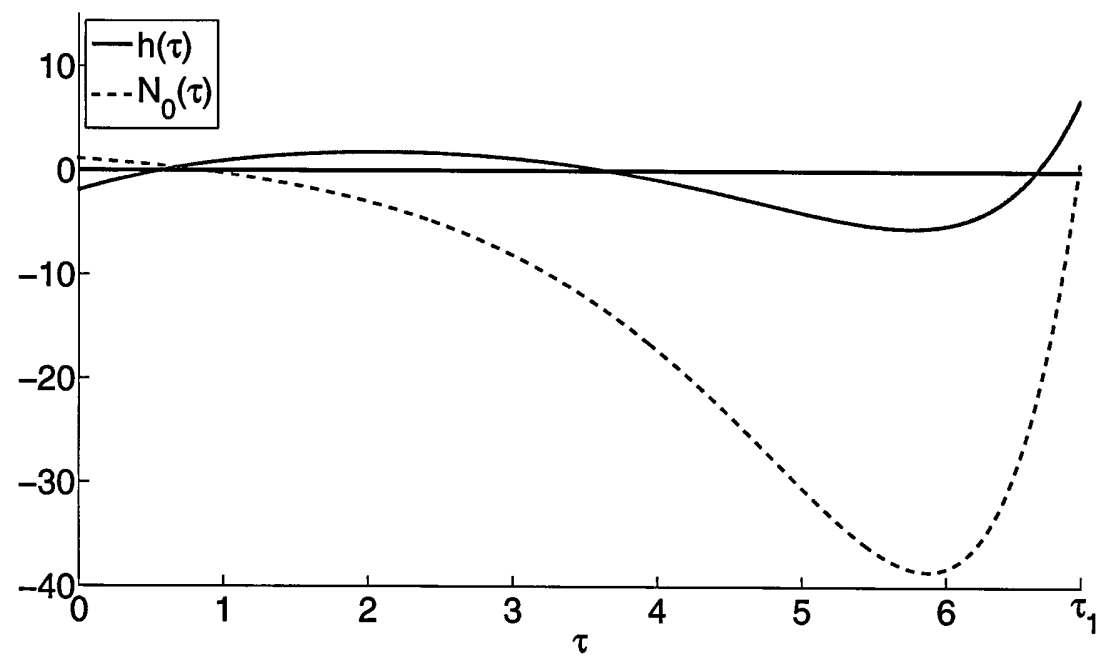

Figure 4.6: A plot of $h(\tau)=e^{\mu_{j} \tau} N^{*}(\tau)-\frac{M}{\mu}$ (see equation (4.21)) and $N_{0}(\tau)$ (see equation (4.18)). As explained in the third and fourth paragraphs of section 4.7 , the conditions of theorem 4.2 hold when $N(0)_{\tau}>0$, and the culling regime of theorem 4.2 increases the mean pest population when $\tau$ is such that $h(\tau)>0$ and decreases it when $\tau$ is such that $h(\tau)<0$. Parameter choice here: $N_{2}=1, M=2, W=10, \mu=1$, and $\mu_{j}=0.1$. In the plot, $\tau_{1}=6.9315 ; h(\tau)=0$ where $\tau=0.6027,3.6373$, and 6.6294; and $N(0)_{\tau}=0$ where $\tau=0.7774$ and 6.9216 .

that theorem 4.2 does not hold unless $N_{0}(\tau)>0$, figure 4.6 shows that the theorem is valid for $\tau \in(0,0.7774)$ and for $\tau \in(6.9216,6.9315)$. Bearing in mind that the culling regime of theorem 4.2 decreases the mean population when $h(\tau)<0$ and increases it when $h(\tau)>0$, figure 4.6 therefore also shows that the regime decreases the mean population for $\tau \in(0,0.6027)$, increases it for $\tau \in(0.6027,0.7774)$, and increases it for $\tau \in(6.9216,6.9315)$. Thus, the precise relationship between the influence of culling on the mean population and the size of the maturation age $\tau$ is not, in general, trivially determined. We will explore this matter further in future research.

\subsection{Discussion}

We have seen evidence, in the form of a simulation, of how an adult impulsive culling regime can maintain a pest population at a level which is higher on average (averaged over the inter-cull period or in the long-term) than it would be in the absence of culling. The simulation involved a Nicholson-type birth function. We considered why culling may benefit a pest in the context of the shape of the birth function. This motivated us to construct a new birth function (a "step" birth function), simpler than a Nicholson function but retaining the features which we felt would permit certain culling regimes to benefit a pest.

We recognised that there are circumstances under which a step birth function can be biologically realistic and the behaviour of a model with such a birth function was analysed in the absence of culling. We also constructed a specific culling regime and 
used it to demonstrate analytically that, for a certain positive finite range of values for the maturation age $\tau$, the regime increases the long-term mean pest population. We proved additionally that the same regime decreases the mean population if the maturation age $\tau$ is sufficiently small. Simulations corroborated our theoretical results.

The drawbacks associated with large-scale applications of pesticides have been described in section 2.3. In the present chapter we have seen that applying pesticides relatively infrequently can benefit a pest. We are in a position to conclude that both over-use and under-use of pesticides can ultimately work against us. It becomes difficult to know precisely what to advocate in terms of the level of application. But we at least feel able to say this: specific real-world pest control problems are best solved individually, by simulation if the problem is analytically intractable. Moreover we have not proven that infrequent culling is always beneficial to a pest. Indeed, we have shown that if the maturation age is suitably small then culling can be detrimental to the pest.

We have seen, by various existence arguments, some of the possible consequences of periodic culling when there is a step birth function. We have by no means acquired a complete understanding of all the possible consequences. There are conditions on the birth function and initial data which we have not considered. Future research could involve seeking a full understanding of the influence of periodic impulsive culling for any continuous unimodal birth function. Non-periodic and non-impulsive culling regimes could also be investigated. Much remains to be explored.

Our work so far has concentrated on models for a creature living in a single region or patch. In the final two pest control chapters we consider models for a creature living on two distinct patches between which migration may occur. 


\title{
Chapter 5
}

\section{Global dynamics of a structured population on two patches}

\author{
"Here is the world, sound as a nut, perfect, not the smallest piece of chaos left, \\ never a stitch nor an end, not a mark of haste, or botching, or second thought; \\ but the theory of the world is a thing of shreds and patches."
}

Ralph Waldo Emerson (1803 - 1882)

\subsection{Introduction}

We now concern ourselves with a model for a single species which has a stagestructured life cycle and which inhabits two regions or "patches". More specifically, the life cycle will consist of two stages, namely a juvenile or immature stage and a mature or adult stage, and migration will take place between the patches in either or both directions.

There are potentially many creatures and situations which these assumptions could describe. The majority of insects lead stage-structured life cycles [17] and insects, like other creatures, will be driven, by instincts to eat and mate, to move around from one place to another. If we wished to think in terms of insect pests, the two patches could represent, for example, neighbouring farms suffering from a common pest infestation, or countries which share both a border and a pest. The regions need not be physically next to each other, because they could also represent ports, or other destinations, connected by trade links which a pest exploits. It is not unusual for rodents or insects to travel with cargo as it is transported across the world by ship or plane. If we can understand when a pest will flourish, we will know when to consider implementing a control stratagem.

So, $\mathrm{Wu}$, and $\mathrm{Zou}$ have previously derived a system of delay differential equations to describe the dynamics of the adult populations on the two patches [129]. Their subsequent analysis of this delay differential system was restricted in several senses. Firstly, they supposed that the model parameters in one patch were identical to the 
corresponding parameters in the other patch. Secondly, they considered only one kind of birth function. Finally, their analysis was limited to local stability arguments for homogeneous equilibria.

In this chapter, we extend the scope of their analysis by establishing numerous global results. We will not always assume the patches are identical and we will consider three different kinds of birth function, namely the linear, Nicholson, and Allee birth functions described in subsection 3.2.1. In particular we discover conditions on the model parameters such that extinction will occur on both patches. We also find conditions on the parameters, and additionally in some cases the initial conditions, such that the creature will remain endemic on both patches.

This chapter has the following format. In section 5.2 we describe the model derived by So, $\mathrm{Wu}$, and $\mathrm{Zou}$, and prove that it satisfies positivity. In section 5.3 we establish conditions for extinction on both patches. In section 5.4 we show that minimum viable populations exist on both patches when the birth functions on both patches are of Allee type. We prove in section 5.5 that the populations are bounded when the birth functions are bounded. In section 5.6 we establish conditions for endemicity on both patches. Simulations are included in section 5.7 and a discussion in section 5.8 ends the chapter.

\subsection{The model}

We model a single species whose life pattern can be split into two distinct developmental stages, namely a juvenile or immature stage and an adult or mature stage. Only the adult stage can reproduce. Assume births and natural deaths occur continuously in time. Assume also that an individual becomes mature on reaching age $\tau$ where $\tau$ is a positive constant called the maturation age, provided the individual lives that long.

Imagine that we have two distinct populations of this species, living on different patches, patch 0 and patch 1 . We may refer to the patches in the order 0 then 1 , or 1 then 0 , if we label the patches as $i$ and $1-i$ and let $i=0$ or $i=1$. This freedom of labelling will be useful.

Let $u_{i}(t, a)$ denote the density of individuals at time $t(t \geq 0)$ of age $a(0 \leq a<\infty)$ on patch $i(i=0,1)$. Then $w_{i}(t)=\int_{\tau}^{\infty} u_{i}(t, a) d a$ is the total number of adults at time $t$ on patch $i(i=0,1)$ and, since only adults can reproduce, $u_{i}(t, 0)=b_{i}\left(w_{i}(t)\right)$, where $b_{i}(w)$ is the birth rate of the population on the $i$-th patch. Since the birth rate on each patch is assumed to depend only on the adult population on that patch and not explicitly on time, we have in mind species that inhabit tropical regions where conditions are conducive to year-round reproduction.

Both immatures and matures can migrate from one patch to the other, in either direction. Assume there is no loss during migration, that is, all of those which leave patch $i$ arrive at patch $1-i$ safely. The migration rates are assumed to satisfy specific functions. Death rates for immatures and matures need not be the same, and death rates in one patch need not be the same as those in the other.

To be specific, let $d_{i}(a)$ be the per capita death rate of individuals of age $a$ in patch $i$ and let $D_{i}(a) u_{i}(t, a)$ denote the dispersal of the species at age $a$ from patch $i$ to patch $1-i(i=0,1)$. (So we may think of $D_{i}(a)$ as a per capita migration rate.) For $i=0,1$, 
assume that

$$
d_{i}(a)= \begin{cases}d_{i, I}(a)=d_{I}(a) & \text { for } 0 \leq a \leq \tau \\ d_{i, M}(a) & \text { for } a>\tau\end{cases}
$$

and

$$
D_{i}(a)= \begin{cases}D_{i, I}(a)=D_{i}(a) & \text { for } 0 \leq a \leq \tau, \\ D_{i, M}(a) & \text { for } a>\tau .\end{cases}
$$

Notice that the immature death rate is assumed independent of the patch. This is sensible if the conditions affecting immature survival are similar on both patches, as they may be expected to be on neighbouring farms, say. Suppose further that the mature death rates $d_{i, M}(a)=d_{i, M}$ and mature migration rates $D_{i, M}(a)=D_{i, M}$ $(i=0,1)$ are all constants, no pair of which are necessarily equal.

Using ideas from Metz and Diekmann [107] on modelling age-structured populations with migration, and following an approach by Smith [127] whilst making the assumptions described above, So, Wu, and Zou [129] found that the adult populations $w_{i}(t)$ satisfy the following system:

$$
\begin{aligned}
\frac{d w_{i}(t)}{d t}= & -d_{i, M} w_{i}(t)+D_{1-i, M} w_{1-i}(t)-D_{i, M} w_{i}(t) \\
& +e^{*}\left[1-\int_{0}^{\tau} e^{-\int_{\theta}^{\tau} \hat{D}(a) d a} D_{i}(\theta) d \theta\right] b_{i}\left(w_{i}(t-\tau)\right) \\
& +e^{*}\left[\int_{0}^{\tau} e^{-\int_{\theta}^{\tau} \hat{D}(a) d a} D_{1-i}(\theta) d \theta\right] b_{1-i}\left(w_{1-i}(t-\tau)\right),
\end{aligned}
$$

for $t \geq 0$, for $i=0,1$, and where $e^{*}=e^{-\int_{0}^{\tau} d_{I}(a) d a}$ and $\hat{D}(a)=D_{i}(a)+D_{1-i}(a)$. Notice that the coefficients of $w_{i}(t), w_{1-i}(t), b_{i}\left(w_{i}(t-\tau)\right)$, and $b_{1-i}\left(w_{1-i}(t-\tau)\right)$ are all constant.

The terms in (5.3) may be interpreted ecologically. The net rate of change at time $t$ of the adult population in patch $i$, namely $\frac{d w_{i}(t)}{d t}$, is the rate of new entries minus the rate of departure. But the rate of new entries into the adult population on patch $i$ at time $t$ is the number of matures migrating into patch $i$ from patch $1-i$ (which is $\left.D_{1-i, M} w_{1-i}(t)\right)$; plus the number of immatures born in patch $i$ becoming mature at time $t$ (which is $b_{i}\left(w_{i}(t-\tau)\right)$ ), scaled by the proportion that have survived to maturity (which is $e^{*}$ ) as well as by the proportion that have not at time $t$ migrated out of patch $i$ (which is $1-\int_{0}^{\tau} e^{-\int_{\theta}^{\tau} \hat{D}(a) d a} D_{i}(\theta) d \theta$ ); plus the number of immatures born in patch $1-i$ becoming mature at time $t$ (which is $b_{1-i}\left(w_{1-i}(t-\tau)\right)$ ), scaled by the proportion that have survived to maturity (which is $e^{*}$ ) as well as by the proportion that have at time $t$ migrated into patch $i$ (which is $\int_{0}^{\tau} e^{-\int_{\theta}^{\tau} \hat{D}(a) d a} D_{1-i}(\theta) d \theta$ ). And the rate of departure of individuals from the adult population on patch $i$ at time $t$ is the number of matures migrating out of patch $i$ into patch $1-i$ (which is $D_{i, M} w_{i}(t)$ ); plus the death rate of adults in patch $i$ (which is $d_{i, M} w_{i}(t)$ ). Henceforth we shall use the term "population" as a shorthand for "adult population".

For a sensibly defined population model we need initial data:

$$
w_{i}(t)=\phi_{i}(t) \geq 0 \text { on }[-\tau, 0]
$$

for $i=0,1$. Here $\phi_{i}(t), i=0,1$, is assumed to be finite for $t \in[-\tau, 0]$.

By saying the derivative $\frac{d w_{i}(t)}{d t}$ exists for $t \geq 0$, it should be understood that the left derivative exists at $t=0$ and that the derivative exists for $t>0$. A solution to (5.3), 
subject to (5.4), will exist and be unique if the functions $b_{i}, d_{i}$, and $\phi_{i}$ are suitably sensible. Delay differential systems and their solutions are given a proper discussion in Kuang's book [95]. We will consider three specific birth functions, namely the linear, Nicholson, and Allee birth functions that are described in subsection 3.2.1. When either of the birth functions $b_{i}$ for $i=0,1$ are any of these three types of birth function, then, for continuous non-negative initial data $\phi_{i}(i=0,1)$, the system (5.3) will have a unique solution for $t \geq 0$, and the birth functions will satisfy:

$$
b_{0}(0)=0, \quad b_{1}(0)=0, \quad \text { and, for } w>0, \quad b_{0}(w)>0, \quad b_{1}(w)>0 .
$$

Instead of working with the large expression in (5.3), it will be easier to work with a system with simpler notation. Thus, relabelling the constant coefficients in (5.3), and writing out the system in full (that is, as two equations), we obtain:

$$
\begin{aligned}
& \frac{d w_{0}(t)}{d t}=-A w_{0}(t)+B w_{1}(t)+C b_{0}\left(w_{0}(t-\tau)\right)+D b_{1}\left(w_{1}(t-\tau)\right) \\
& \frac{d w_{1}(t)}{d t}=E w_{0}(t)-F w_{1}(t)+G b_{0}\left(w_{0}(t-\tau)\right)+H b_{1}\left(w_{1}(t-\tau)\right),
\end{aligned}
$$

where $A, B, C, D, E, F, G, H$ are all positive constants, specifically:

$$
\begin{array}{rlrl}
A & =d_{0, M}+D_{0, M} & B & =D_{1, M} \\
C & =e^{*}\left[1-\int_{0}^{\tau} e^{-\int_{\theta}^{\tau} \hat{D}(a) d a} D_{0}(\theta) d \theta\right] & D & =e^{*}\left[\int_{0}^{\tau} e^{-\int_{\theta}^{\tau} \hat{D}(a) d a} D_{1}(\theta) d \theta\right] \\
E & =D_{0, M} & F & =d_{1, M}+D_{1, M} \\
G & =e^{*}\left[\int_{0}^{\tau} e^{-\int_{\theta}^{\tau} \hat{D}(a) d a} D_{0}(\theta) d \theta\right] & H & =e^{*}\left[1-\int_{0}^{\tau} e^{-\int_{\theta}^{\tau} \hat{D}(a) d a} D_{1}(\theta) d \theta\right] .
\end{array}
$$

Notice that $A>E$ and $F>B$. Although it is obvious that $A, B, D, E, F, G$ are all positive, it is less obvious that $C$ and $H$ are positive. To see that $C>0$, note first that $e^{*}>0$ trivially. Next notice that $\int_{0}^{\tau} e^{-\int_{\theta}^{\tau} \hat{D}(a) d a} D_{0}(\theta) d \theta<1$ by the following calculation:

$$
\begin{aligned}
\int_{0}^{\tau} e^{-\int_{\theta}^{\tau} \hat{D}(a) d a} D_{0}(\theta) d \theta & <\int_{0}^{\tau} e^{-\int_{\theta}^{\tau} D_{0}(a) d a} D_{0}(\theta) d \theta \quad \text { since } \hat{D}(a)>D_{0}(a) \\
& =\int_{0}^{\tau} \frac{d}{d \theta}\left\{e^{-\int_{\theta}^{\tau} D_{0}(a) d a}\right\} d \theta \\
& =\left[e^{-\int_{\theta}^{\tau} D_{0}(a) d a}\right]_{\theta=0}^{\theta=\tau} \\
& =1-e^{-\int_{0}^{\tau} D_{0}(a) d a} .
\end{aligned}
$$

Given that $e^{*}>0$ and $\int_{0}^{\tau} e^{-\int_{\theta}^{\tau} \hat{D}(a) d a} D_{0}(\theta) d \theta<1$, it is immediately apparent that $C>0$. Similarly, we can show that $H>0$.

A first step in examining a population model is to show that it is biologically sensible. Thus, the initial data on both patches should be non-negative, and the populations on both patches should remain non-negative for all time $t \geq 0$. The property that $w_{i}(t) \geq 0$ for $t \geq 0$, for $i=0,1$, is called positivity. The special case $w_{i}(t)>0$ for $t \geq 0$ is strict positivity.

Lemma 5.1. The system given by (5.6) and (5.7), subject to (5.4) and (5.5), satisfies positivity. 
Proof. The proof is by the method of steps. In other words, we will show by induction on $j \geq 0$ that $w_{0}(t) \geq 0$ and $w_{1}(t) \geq 0$ for $t \in[j \tau,(j+1) \tau]$.

Basis step $(j=0)$. By the initial data (5.4), and equations (5.5), (5.6) and (5.7), we have, for $t \in[0, \tau]$,

$$
\begin{aligned}
& \frac{d w_{0}(t)}{d t} \geq-A w_{0}(t)+B w_{1}(t) \\
& \frac{d w_{1}(t)}{d t} \geq E w_{0}(t)-F w_{1}(t) .
\end{aligned}
$$

It follows (theorem 1.1, pp. 78-79, [128]) that $w_{0}(t) \geq x_{0}(t)$ and $w_{1}(t) \geq x_{1}(t)$ for $t \in[0, \tau]$ where $x_{0}(0)=0 \leq w_{0}(0)$ and $x_{1}(0)=0 \leq w_{1}(0)$ and where, for $t \in[0, \tau]$, we have:

$$
\begin{aligned}
& \frac{d x_{0}(t)}{d t}=-A x_{0}(t)+B x_{1}(t) \\
& \frac{d x_{1}(t)}{d t}=E x_{0}(t)-F x_{1}(t) .
\end{aligned}
$$

Since $x_{0}(0)=0$ and $x_{1}(0)=0$, a solution to (5.14) and (5.15) is $x_{0}(t) \equiv 0$ and $x_{1}(t) \equiv 0$ for $t \in[0, \tau]$. Since the system given by (5.14) and (5.15) is linear, standard results (see p.106 in [75], for example) ensure that this solution is unique. Hence, for $t \in[0, \tau]$, we have $w_{0}(t) \geq x_{0}(t)=0$ and $w_{1}(t) \geq x_{1}(t)=0$. But this is exactly what is required on the basis step.

Inductive step. This is very similar to the basis step.

\subsection{Extinction}

As we remarked in the last section, we shall suppose that the birth function on patch 0 could be linear, Nicholson, or Allee, and that the birth function on patch 1 could also be linear, Nicholson, or Allee. If both birth functions are of the same type, they need not have the same parameter values. For example, if they are both linear, they need not be the same linear function. There are nine distinct cases, arising from the facts that there are two birth functions (one for each patch), and each can be any of three different types (linear, Nicholson, or Allee). As we stated in section 5.1, the patches could represent regions not physically next to each other, so there is no obvious reason to suppose that the form of the birth function will be the same on both patches.

We shall now prove that the creature can go naturally extinct on both patches simultaneously. We can do this for all nine possible cases with a single result (theorem 5.2 below). Before this result can be stated, we must state a number of other results.

Lemma 5.2. The Nicholson and Allee birth functions can be bounded above by linear functions. In fact, for $w \geq 0$, we have $\lambda_{1} w e^{-\lambda_{2} w} \leq \lambda_{1} w$ and $\alpha_{1} w^{2} e^{-\alpha_{2} w} \leq \frac{\alpha_{1}}{\alpha_{2} e} w$.

It is trivial to prove lemma 5.2, so we shall not include a proof. 
Theorem 5.1. Suppose, for any positive constant $W$, that $0 \leq N(t) \leq W$ for $t \in[-\tau, 0]$ and that, for $t \geq 0$, we have:

$$
\frac{d N(t)}{d t} \leq \lambda N(t-\tau)-\mu N(t)
$$

where $0<\lambda<\mu$. Then if $N(t)$ satisfies positivity, we have $N(t) \rightarrow 0$ as $t \rightarrow \infty$.

Proof. Observe (by theorem 1.1, pp. 78-79, [128]) that $N(t) \leq N_{1}(t)$ for $t \geq-\tau$ if we suppose that $N_{1}(t)=N(t)$ for $t \in[-\tau, 0]$ and that, for $t \geq 0$ :

$$
\frac{d N_{1}(t)}{d t}=\lambda N_{1}(t-\tau)-\mu N_{1}(t)
$$

But theorem 3.1 says that if $0<\lambda<\mu$, then the function $N_{1}(t)$ just defined tends to zero as $t \rightarrow \infty$. Since we are assuming that $N(t)$ satisfies positivity, we then have $0 \leq N(t) \leq N_{1}(t) \rightarrow 0$ as $t \rightarrow \infty$. In other words, $N(t) \rightarrow 0$ as $t \rightarrow \infty$.

Now we can prove that the pest can go naturally extinct on both patches simultaneously:

Theorem 5.2. In the system given by (5.6) and (5.7), subject to (5.4), suppose the birth function $b_{0}$ is linear, Nicholson, or Allee, and suppose the birth function $b_{1}$ is linear, Nicholson, or Allee and is not necessarily identical to $b_{0}$. Then $b_{i}(w) \leq \Lambda_{i} w$ for $i=0,1$, where $\Lambda_{0}$ and $\Lambda_{1}$ are positive constants. Note that $C+G=D+H=e^{*}$ using (5.9) and (5.11) and let $\lambda=e^{*} \max \left\{\Lambda_{0}, \Lambda_{1}\right\}$. Also let $\mu=\min (A-E, F-B)=\min \left(d_{0, m}, d_{1, m}\right)$. Notice that $\mu>0$. Assume $\lambda<\mu$. Then both $w_{0}(t) \rightarrow 0$ and $w_{1}(t) \rightarrow 0$ as $t \rightarrow \infty$.

Proof. It is clear that the birth functions can be bounded above by linear functions by lemma 5.2.

Add together equations (5.6) and (5.7) to find that:

$$
\begin{aligned}
\frac{d w_{0}(t)}{d t}+\frac{d w_{1}(t)}{d t} \leq \quad & (A-E) w_{0}(t)-(F-B) w_{1}(t) \\
& +(C+G) \Lambda_{0} w_{0}(t-\tau)+(D+H) \Lambda_{1} w_{1}(t-\tau) .
\end{aligned}
$$

Let $N(t)=w_{0}(t)+w_{1}(t)$ and notice that, by the positivity of $w_{0}(t)$ and $w_{1}(t)$ (by lemma 5.1), then $N(t) \geq 0$ for $t \geq-\tau$. Using the definitions of $\lambda$ and $\mu$ given in the statement of the theorem, we see that:

$$
\frac{d N(t)}{d t} \leq \lambda N(t-\tau)-\mu N(t)
$$

But now, by equation (5.19), the assumption that $\lambda<\mu$, the fact that the initial data in (5.4) is assumed to be non-negative and finite, and using theorem 5.1, we see that $w_{0}(t)+w_{1}(t)=N(t) \rightarrow 0$ as $t \rightarrow \infty$. Since $w_{0}(t)$ and $w_{1}(t)$ satisfy positivity individually (lemma 5.1), we may conclude that both $w_{0}(t) \rightarrow 0$ and $w_{1}(t) \rightarrow 0$ as $t \rightarrow \infty$. 
We noted in the paragraph after (5.3) that $e^{*}$ may be interpreted as the proportion of immatures that survive to maturity. It may also then be interpreted as the probability of survival to maturity. But then theorem 5.2 effectively says that extinction on both patches will occur if the birth rates on both patches, scaled by the probability of survival to maturity, are bounded above by the minimum adult death rate. Such a result is in keeping with intuition.

\subsection{Minimum viable population for Allee births}

We mentioned in subsection 3.2.1 that the Allee birth function may be associated with a phenomenon called the Allee effect ( [45] and p. 23 in [55]) in which a population can be low enough as to be unsustainable. Such a phenomenon may occur naturally because in a small population there may be little group defence or it may be difficult for an individual to find a mate since the population density may be lower. The Allee effect has been seen to hold for a closed population on a single patch when there is no delay in the birth term (lemma 3.5) or when there is a delay (theorem 3.13). We add to those results by proving in this section that there is an Allee effect in the two patch model of this chapter (equations (5.6), (5.7), and (5.4)) when the birth function on each patch is of Allee type. (It is trivial to prove that an Allee effect will hold in this model when the delay $\tau$ is set equal to zero.)

Our main result in this section is corollary 5.1. Before we can prove this, we need to establish a lemma and a theorem.

Lemma 5.3. Let $b(w)=w^{2} e^{-\alpha w}$. Then $b(w)$ is maximum over all $w \geq 0$ where $w=\frac{2}{\alpha}$. Suppose $x \geq 0, y \geq 0$, and $x+y \leq \frac{2}{\alpha}$. Then

$$
x^{2} e^{-\alpha x}+y^{2} e^{-\alpha y} \leq 2(x+y)^{2} e^{-\alpha(x+y)} .
$$

Proof. First observe that $\frac{d b(w)}{d w}=w(2-\alpha w) e^{-\alpha w}$. It follows that if $w \geq 0$ then $b(w)$ is maximum where $w=\frac{2}{\alpha}$ and that, if $0<w<\frac{2}{\alpha}$, then $b(w)$ is monotonic increasing. But then, since $x \geq 0, y \geq 0$, and $x+y \leq \frac{2}{\alpha}$, we have $x^{2} e^{-\alpha x} \leq(x+y)^{2} e^{-\alpha(x+y)}$ and $y^{2} e^{-\alpha y} \leq(x+y)^{2} e^{-\alpha(x+y)}$. Inequality (5.20) follows at once.

Theorem 5.3. Suppose $w_{0}(t)$ and $w_{1}(t)$ satisfy (5.6) and (5.7), subject to (5.4). Suppose the birth functions on both patches 0 and 1 are of Allee type. Specifically let $b_{0}(w)=\alpha_{1} w^{2} e^{-\alpha_{2} w}$ and $b_{1}(w)=\alpha_{3} w^{2} e^{-\alpha_{4} w}$.

Define the following:

$$
\begin{aligned}
P & =\min (A-E, F-B)=\min \left(d_{0, m}, d_{1, m}\right)=\text { minimum adult death rate } \\
Q & =\max \left((C+G) \alpha_{1},(D+H) \alpha_{3}\right) \\
\alpha & =\min \left(\alpha_{2}, \alpha_{4}\right)
\end{aligned}
$$

Let $K=\min \left(\frac{P}{2 Q}, \frac{2}{\alpha}\right)$.

Suppose that $w_{0}(t)+w_{1}(t) \leq K$ for $t \in[-\tau, 0]$. Then $w_{0}(t)+w_{1}(t) \leq K$ for all $t \geq 0$. 
Proof. We show by induction on $j \geq 0$ that $w_{0}(t)+w_{1}(t) \leq K$ for $t \in[j \tau,(j+1) \tau]$.

Basis step $(j=0)$. Add together equations (5.6) and (5.7) to obtain, for $t \geq 0$,

$$
\begin{gathered}
\frac{d w_{0}(t)}{d t}+\frac{d w_{1}(t)}{d t}=-(A-E) w_{0}(t)-(F-B) w_{1}(t)+(C+G) b_{0}\left(w_{0}(t-\tau)\right) \\
+(D+H) b_{1}\left(w_{1}(t-\tau)\right)
\end{gathered}
$$

But then, using the various definitions in the statement of the theorem as well as positivity (lemma 5.1), we have, for $t \geq 0$,

$$
\begin{gathered}
\frac{d}{d t}\left(w_{0}(t)+w_{1}(t)\right) \leq Q\left(\left(w_{0}(t-\tau)\right)^{2} e^{-\alpha w_{0}(t-\tau)}+\left(w_{1}(t-\tau)\right)^{2} e^{-\alpha w_{1}(t-\tau)}\right) \\
-P\left(w_{0}(t)+w_{1}(t)\right) .
\end{gathered}
$$

By assumption, $w_{0}(t)+w_{1}(t) \leq K \leq \frac{2}{\alpha}$ for $t \in[-\tau, 0]$. Hence $w_{0}(t-\tau)+w_{1}(t-\tau) \leq$ $K \leq \frac{2}{\alpha}$ for $t \in[0, \tau]$. Using this, and since the initial data is non-negative by (5.4) we have, using lemma 5.3 , that, for $t \in[0, \tau]$,

$$
\begin{aligned}
& \left(w_{0}(t-\tau)\right)^{2} e^{-\alpha w_{0}(t-\tau)}+\left(w_{1}(t-\tau)\right)^{2} e^{-\alpha w_{1}(t-\tau)} \\
\leq & 2\left(w_{0}(t-\tau)+w_{1}(t-\tau)\right)^{2} e^{-\alpha\left(w_{0}(t-\tau)+w_{1}(t-\tau)\right)} .
\end{aligned}
$$

Let $N(t)=w_{0}(t)+w_{1}(t)$. Then by (5.25) and (5.26) we have, for $t \in[0, \tau]$,

$$
\frac{d N(t)}{d t} \leq 2 Q(N(t-\tau))^{2} e^{-\alpha N(t-\tau)}-P N(t) .
$$

Now we have seen in lemma 5.3 that the function $w^{2} e^{-\alpha w}$ is monotonic increasing for $0<w<\frac{2}{\alpha}$. Also, by assumption, $0 \leq N(t)=w_{0}(t)+w_{1}(t) \leq K \leq \frac{2}{\alpha}$ for $t \in[-\tau, 0]$, so that $0 \leq N(t-\tau) \leq K \leq \frac{2}{\alpha}$ for $t \in[0, \tau]$. Hence by (5.27), we have, for $t \in[0, \tau]$,

$$
\frac{d N(t)}{d t} \leq 2 Q K^{2} e^{-\alpha K}-P N(t)
$$

But then $N(t) \leq N_{1}(t)$ for $t \in[0, \tau]$ where $N_{1}(0)=K \geq N(0)$ and, for $t \in[0, \tau]$, we have:

$$
\frac{d N_{1}(t)}{d t}=2 Q K^{2} e^{-\alpha K}-P N_{1}(t) .
$$

Solving for $N_{1}(t)$ gives, for $t \in[0, \tau]$,

$$
N(t) \leq N_{1}(t)=\frac{2 Q K^{2}}{P} e^{-\alpha K}+K\left(1-\frac{2 Q K}{P} e^{-\alpha K}\right) e^{-P t}
$$

Notice that $N_{1}(t)$ is non-increasing on $[0, \tau]$ if $1-\frac{2 Q K}{P} e^{-\alpha K} \geq 0$, which is certainly true if $K \leq \frac{P}{2 Q}$, which is true by assumption. Therefore, since $N_{1}(0)=K$, we must have $w_{0}(t)+w_{1}(t)=N(t) \leq N_{1}(t) \leq K$ for $t \in[0, \tau]$, which is the result required on the basis step.

Inductive step. The method is the same as on the basis step. 
Corollary 5.1. Make the assumptions in theorem 5.3, except for the initial data for which we make a slightly stricter assumption, namely that $w_{0}(t)+w_{1}(t)<\psi$ for $t \in[-\tau, 0]$ where $\psi$ is a positive constant defined as follows:

(i) If $2 Q<e \alpha P$ then let $\psi=K=\min \left\{\frac{P}{2 Q}, \frac{2}{\alpha}\right\}$. (Here $Q, \alpha, P$, and $K$ are quantities defined in theorem 5.3.)

(ii) If $2 Q \geq e \alpha P$ then a positive solution to $2 Q N^{2} e^{-\alpha N}=P N$ exists by lemma 3.5. Let $N^{*}$ be the least such solution and let $\psi=\min \left\{K, N^{*}\right\}=\min \left\{\frac{P}{2 Q}, \frac{2}{\alpha}, N^{*}\right\}$.

Then $w_{0}(t) \rightarrow 0$ and $w_{1}(t) \rightarrow 0$ as $t \rightarrow \infty$.

Proof. By theorem 5.3, we know that $w_{0}(t)+w_{1}(t) \leq K \leq \frac{2}{\alpha}$ for $t \geq-\tau$. Also, by positivity (lemma 5.1) we know that $w_{0}(t) \geq 0$ and $w_{1}(t) \geq 0$ for $t \geq-\tau$. Then, by lemma 5.3, we have, for $t \geq 0$ :

$$
\begin{aligned}
& \left(w_{0}(t-\tau)\right)^{2} e^{-\alpha w_{0}(t-\tau)}+\left(w_{1}(t-\tau)\right)^{2} e^{-\alpha w_{1}(t-\tau)} \\
\leq & 2\left(w_{0}(t-\tau)+w_{1}(t-\tau)\right)^{2} e^{-\alpha\left(w_{0}(t-\tau)+w_{1}(t-\tau)\right)} .
\end{aligned}
$$

As in the proof of theorem 5.3, we find that equations (5.24) and (5.25) hold for $t \geq 0$. But by equations (5.25) and (5.31), we may write, for $t \geq 0$, that

$$
\begin{gathered}
\frac{d w_{0}(t)}{d t}+\frac{d w_{1}(t)}{d t} \leq 2 Q\left(w_{0}(t-\tau)+w_{1}(t-\tau)\right)^{2} e^{-\alpha\left(w_{0}(t-\tau)+w_{1}(t-\tau)\right)} \\
-P\left(w_{0}(t)+w_{1}(t)\right)
\end{gathered}
$$

Let $N(t)=w_{0}(t)+w_{1}(t)$. Then (5.32) becomes

$$
\frac{d N(t)}{d t} \leq 2 Q(N(t-\tau))^{2} e^{-\alpha N(t-\tau)}-P N(t) \text { for } t \geq 0 .
$$

But then (using theorem 1.1, pp. 78-79, [128]) we can say that $N(t) \leq N_{1}(t)$ for $t \geq 0$ where $N_{1}(t)=N(t)$ for $t \in[-\tau, 0]$, and where

$$
\frac{d N_{1}(t)}{d t}=2 Q\left(N_{1}(t-\tau)\right)^{2} e^{-\alpha N_{1}(t-\tau)}-P N_{1}(t) \text { for } t \geq 0 .
$$

Now the initial data is $0 \leq N_{1}(t)=N(t)=w_{0}(t)+w_{1}(t)<\psi$ for $t \in[-\tau, 0]$ where $\psi$ is defined according to whether $P<\frac{2 Q}{\alpha e}$ or $P \geq \frac{2 Q}{\alpha e}$. If $P<\frac{2 Q}{\alpha e}$ we immediately deduce by the initial data, by (5.34), and by theorem 3.10 that $N_{1}(t) \rightarrow 0$ as $t \rightarrow \infty$. But if $P \geq \frac{2 Q}{\alpha e}$, we deduce by the initial data, by (5.34), and by theorem 3.13 that $N_{1}(t) \rightarrow 0$ as $t \rightarrow \infty$.

Thus $w_{0}(t)+w_{1}(t)=N(t) \leq N_{1}(t) \rightarrow 0$ as $t \rightarrow \infty$, so that $w_{0}(t)+w_{1}(t) \rightarrow 0$ as $t \rightarrow \infty$ since $w_{0}(t)+w_{1}(t)$ satisfies positivity by lemma 5.1. But then, since $w_{0}(t)$ and $w_{1}(t)$ satisfy positivity individually (lemma 5.1 ), we must have $w_{0}(t) \rightarrow 0$ and $w_{1}(t) \rightarrow 0$ as $t \rightarrow \infty$. 
From corollary 5.1, an Allee effect holds for a creature with a stage-structured life cycle inhabiting two patches on each of which the birth function is of Allee type. Minimum viable populations exist on the patches. In fact, if the combined population from both patches is for any reason reduced below the value $\psi$ defined in corollary 5.1 for at least $\tau$ consecutive time units, then extinction on both patches becomes inevitable. Such an observation will allow us to construct, in the next chapter, adult impulsive culling regimes that eradicate the pest on both patches with only finitely many culls on each patch. But it also serves as a warning. Pest control measures (such as the application of pesticides) that reduce populations of non-target species may wipe them out if an Allee effect holds. Habitat destruction may have a similar impact.

\subsection{Population boundedness when birth functions are bounded}

Theorem 5.4. In the system given by (5.6) and (5.7), subject to (5.4) and (5.5), suppose that both the birth functions, $b_{0}(w)$ and $b_{1}(w)$, are bounded above for $w \geq 0$. Then the populations on both patches are bounded above for $t \geq 0$.

Proof. We know that $w_{0}(t)$ and $w_{1}(t)$ satisfy positivity for $t \geq-\tau$ by lemma 5.1. But then, by the assumption in the statement of the theorem, $b_{0}\left(w_{0}(t-\tau)\right)$ and $b_{1}\left(w_{1}(t-\tau)\right)$ are bounded above for $t \geq 0$. So we can write, for $t \geq 0$, that $b_{0}\left(w_{0}(t-\tau)\right) \leq W_{0}$ and $b_{1}\left(w_{1}(t-\tau)\right) \leq W_{1}$ for positive constants $W_{0}, W_{1}$. Then, adding together (5.6) and (5.7) gives, for $t \geq 0$,

$$
\frac{d}{d t}\left(w_{0}(t)+w_{1}(t)\right) \leq-(A-E) w_{0}(t)-(F-B) w_{1}(t)+(C+G) W_{0}+(D+H) W_{1} .
$$

Let $N(t)=w_{0}(t)+w_{1}(t)$. Also let $K_{0}=\min (A-E, F-B)=\min \left(d_{0, m}, d_{1, m}\right)$ and $K_{1}=(C+G) W_{0}+(D+H) W_{1}$. Then by (5.35), we have:

$$
\frac{d N(t)}{d t} \leq K_{1}-K_{0} N(t) \text { for } t \geq 0 .
$$

It follows that $N(t) \leq N_{1}(t)$ for $t \geq 0$ where $N_{1}(0)=w_{0}(0)+w_{1}(0)=N(0)$ and where $\frac{d N_{1}(t)}{d t}=K_{1}-K_{0} N_{1}(t)$ for $t \geq 0$. Solving for $N_{1}(t)$ reveals that

$$
N(t) \leq N_{1}(t)=\frac{K_{1}}{K_{0}}+\left(w_{0}(0)+w_{1}(0)-\frac{K_{1}}{K_{0}}\right) e^{-K_{0} t} \text { for } t \geq 0 .
$$

Hence $w_{0}(t)+w_{1}(t)=N(t) \leq \max \left(\frac{K_{1}}{K_{0}}, w_{0}(0)+w_{1}(0)\right)$ for $t \geq 0$. Since $w_{0}(t)$ and $w_{1}(t)$ satisfy positivity, we conclude that $w_{0}(t) \leq \max \left(\frac{K_{1}}{K_{0}}, w_{0}(0)+w_{1}(0)\right)$ and $w_{1}(t) \leq \max \left(\frac{K_{1}}{K_{0}}, w_{0}(0)+w_{1}(0)\right)$ for $t \geq 0$. Certainly, then, $w_{0}(t)$ and $w_{1}(t)$ are bounded above.

Corollary 5.2. In the system given by (5.6) and (5.7), subject to (5.4), suppose the birth function $b_{0}$ is either of Nicholson or Allee type. Suppose also that the birth function $b_{1}$ is either of Nicholson or Allee type. Then the populations on both patches are bounded above for $t \geq 0$. 
Proof. It is obvious a birth function of Nicholson or Allee type satisfies (5.5). Thus the result will follow immediately by theorem 5.4 if we can show that a birth function $b(w)$ of Nicholson-type or Allee-type is bounded above for $w \geq 0$. But a birth function of Nicholson-type, say $\lambda_{1} w e^{-\lambda_{2} w}$, is trivially seen, by elementary calculus, to be bounded above for $w \geq 0$, with the function attaining its maximum value where $w=\frac{1}{\lambda_{2}}$. Similarly a birth function of Allee-type, say $\alpha_{1} w^{2} e^{-\alpha_{2} w}$, is easily seen to be bounded above for $w \geq 0$, with the function attaining its maximum value where $w=\frac{2}{\alpha_{2}}$.

Corollary 5.2 is valuable because it shows the system given by (5.6) and (5.7), subject to (5.4), can be valid for all time $t \geq 0$ when the birth functions are either Nicholson or Allee. If the population on either patch were to diverge to infinity, we would be inclined to question the long-term validity of the model.

In contrast to corollary 5.2, we will discover in theorem 5.6 that when at least one of the birth functions is linear, then the model may allow both $w_{0}(t) \rightarrow \infty$ and $w_{1}(t) \rightarrow \infty$ as $t \rightarrow \infty$.

\subsection{Endemicity}

By endemicity we mean persistence, or the opposite of extinction. Thus if a population $w(t)$ remains endemic, it must persist in the sense that $w(t) \not t 0$ as $t \rightarrow \infty$.

\subsubsection{Linear births}

In this subsection we show that the creature can remain endemic on both patches simultaneously when at least one of the birth functions is linear. First we need a preliminary result.

Theorem 5.5. Suppose, for any positive constant $M$, that $N(t) \geq M$ for $t \in[-\tau, 0]$ and that, for $t \geq 0$, we have:

$$
\frac{d N(t)}{d t} \geq \lambda N(t-\tau)-\mu N(t)
$$

where $0<\mu<\lambda$. Then $N(t) \rightarrow \infty$ as $t \rightarrow \infty$.

Proof. Observe (by theorem 1.1, pp. 78-79, [128]) that $N(t) \geq N_{1}(t)$ for $t \geq-\tau$ if we suppose that $N_{1}(t)=N(t) \geq M$ for $t \in[-\tau, 0]$ and that, for $t \geq 0$ :

$$
\frac{d N_{1}(t)}{d t}=\lambda N_{1}(t-\tau)-\mu N_{1}(t) .
$$

But theorem 3.3 says that if $0<\mu<\lambda$, then the function $N_{1}(t)$ just defined tends to infinity as $t \rightarrow \infty$. But then $N(t) \geq N_{1}(t) \rightarrow \infty$ as $t \rightarrow \infty$. 
Theorem 5.6. Let $w_{0}(t)$ and $w_{1}(t)$ satisfy (5.6) and (5.7), subject to (5.4). Suppose, for $i=0,1$, that the birth functions $b_{i}(\cdot)$ satisfy $(5.5)$ and that $w_{i}(t) \geq M$ for $t \in[-\tau, 0]$ where $M$ is a positive constant.

Assume that at least one of the following holds:

(1) the birth function $b_{0}$ is linear, specifically $b_{0}(w)=\Lambda_{0} w$ where $\Lambda_{0}>\frac{A}{C}$

(2) the birth function $b_{1}$ is linear, specifically $b_{1}(w)=\Lambda_{1} w$ where $\Lambda_{1}>\frac{F}{H}$.

Then $w_{i}(t) \rightarrow \infty$ as $t \rightarrow \infty$ for $i=0,1$.

Proof. Suppose that assumption (1) holds. The proof where assumption (2) holds is very similar. The system given by (5.6) and (5.7) becomes, for $t \geq 0$ :

$$
\begin{aligned}
& \frac{d w_{0}(t)}{d t}=-A w_{0}(t)+B w_{1}(t)+C \Lambda_{0} w_{0}(t-\tau)+D b_{1}\left(w_{1}(t-\tau)\right) \\
& \frac{d w_{1}(t)}{d t}=E w_{0}(t)-F w_{1}(t)+G \Lambda_{0} w_{0}(t-\tau)+H b_{1}\left(w_{1}(t-\tau)\right) .
\end{aligned}
$$

The system satisfies positivity by lemma 5.1. But then it follows (using (5.5) and theorem 1.1, pp. 78-79, [128]) that, for $i=0,1$, we have $w_{i}(t) \geq x_{i}(t)$ for $t \geq 0$, where $x_{i}(t)=M \leq w_{i}(t)$ for $t \in[-\tau, 0]$ and where, for $t \geq 0$, we have:

$$
\begin{aligned}
& \frac{d x_{0}(t)}{d t}=-A x_{0}(t)+C \Lambda_{0} x_{0}(t-\tau) \\
& \frac{d x_{1}(t)}{d t}=E x_{0}(t)-F x_{1}(t) .
\end{aligned}
$$

Clearly we can determine $x_{0}(t)$ from (5.42) alone. But by theorem 5.5 and the fact that $\Lambda_{0}>\frac{A}{C}$ by assumption (1), we see that $x_{0}(t) \rightarrow \infty$ as $t \rightarrow \infty$.

We will have $x_{1}(t) \rightarrow \infty$ if, for any $W>0$, there exists $T>0$ such that, for all $t \geq T$, then $x_{1}(t) \geq W$. So pick any $W>0$. Since $x_{0}(t) \rightarrow \infty$ there exists $T^{*}>0$ such that, for all $t \geq T^{*}$, then $x_{0}(t) \geq \frac{2 F W}{E}$. But then, by (5.43), we find that, for $t \geq T^{*}$,

$$
\frac{d x_{1}(t)}{d t} \geq 2 F W-F x_{1}(t)
$$

But then $x_{1}(t) \geq y(t)$ for $t \geq T^{*}$ where $y\left(T^{*}\right)=x_{1}\left(T^{*}\right)$ and, for $t \geq T^{*}$,

$$
\frac{d y(t)}{d t}=2 F W-F y(t)
$$

Solving for $y(t)$ reveals that, for $t \geq T^{*}$,

$$
x_{1}(t) \geq y(t)=2 W+e^{-F\left(t-T^{*}\right)}\left(x_{1}\left(T^{*}\right)-2 W\right) .
$$

Since $e^{-F\left(t-T^{*}\right)} \rightarrow 0$ as $t \rightarrow \infty$, we clearly have, for $t$ large enough, say $t>T$, that $e^{-F\left(t-T^{*}\right)}\left(x_{1}\left(T^{*}\right)-2 W\right) \geq-W$. For any $W>0$, then, we may deduce from (5.46) that there exists $T>0$ such that, for $t \geq T$,

$$
x_{1}(t) \geq y(t) \geq W .
$$


In other words, $x_{1}(t) \rightarrow \infty$ as $t \rightarrow \infty$. Thus for $i=0,1$ we have $w_{i}(t) \geq x_{i}(t) \rightarrow \infty$ as $t \rightarrow \infty$, so $w_{i}(t) \rightarrow \infty$ as $t \rightarrow \infty$.

As we remarked after corollary 5.2 , it is unrealistic to expect populations to diverge to infinity in real life. Therefore the assumptions in theorem 5.6 are not likely to be valid for all time. But over a limited time-scale, theorem 5.6 carries weight.

\subsubsection{Nicholson or Allee births}

In this subsection we show that the creature can remain endemic on both patches simultaneously when either birth function is either Nicholson or Allee and when the model parameters (except perhaps those defining the birth functions) are identical. If the patches are identical (except possibly the birth functions) we may simplify the parameters and equations defining the model. Thus, from section 5.2, we know that the mature death rates must be equal $\left(d_{0, M}=d_{1, M}=d_{M}\right)$, that the immature migration rates must be equal $\left(D_{0}(a)=D_{1}(a)=D_{I}(a)\right.$ for $\left.0 \leq a \leq \tau\right)$, and that the mature migration rates must be equal $\left(D_{0, M}=D_{1, M}=D_{M}\right)$. We were already assuming that the immature death functions were identical and equal to $d_{I}(a)$ for $0 \leq a \leq \tau$. Consequently the equations describing the evolution of the system, namely (5.6) and (5.7), become:

$$
\begin{aligned}
& \frac{d w_{0}(t)}{d t}=-A w_{0}(t)+B w_{1}(t)+C b_{0}\left(w_{0}(t-\tau)\right)+D b_{1}\left(w_{1}(t-\tau)\right) \\
& \frac{d w_{1}(t)}{d t}=B w_{0}(t)-A w_{1}(t)+D b_{0}\left(w_{0}(t-\tau)\right)+C b_{1}\left(w_{1}(t-\tau)\right)
\end{aligned}
$$

where $A=d_{M}+D_{M}, B=D_{M}, C=e^{*}\left(1-r^{*}\right)$, and $D=e^{*} r^{*}$, where $e^{*}=e^{-\int_{0}^{\tau} d_{I}(a) d a}$ and

$$
\begin{aligned}
r^{*} & =\int_{0}^{\tau} e^{-2 \int_{\theta}^{\tau} D_{I}(a) d a} D_{I}(\theta) d \theta=\frac{1}{2} \int_{0}^{\tau} \frac{d}{d \theta}\left(e^{-2 \int_{\theta}^{\tau} D_{I}(a) d a}\right) d \theta \\
& =\frac{1}{2}\left[e^{-2 \int_{\theta}^{\tau} D_{I}(a) d a}\right]_{0}^{\tau}=\frac{1}{2}\left[1-e^{-2 \int_{0}^{\tau} D_{I}(a) d a}\right] .
\end{aligned}
$$

Notice that we have $A>B$.

For the rest of this subsection, we will concern ourselves with the following "birth options":

(B1) identical Nicholson birth functions: $b_{0}(w)=b_{1}(w)=\lambda_{0} w e^{-\lambda_{1} w}$

(B2) identical Allee birth functions: $b_{0}(w)=b_{1}(w)=\alpha_{0} w^{2} e^{-\alpha_{1} w}$

(B3) $b_{0}$ Nicholson and $b_{1}$ Allee: $b_{0}(w)=\lambda_{0} w e^{-\lambda_{1} w}$ and $b_{1}(w)=\alpha_{0} w^{2} e^{-\alpha_{1} w}$

The possibility that $b_{0}$ is Allee and $b_{1}$ is Nicholson can be studied in the same way as we will study option (B3).

It is our intention to find positive constants $L$ and $U$ with $L<U$ such that if $w_{i}(t) \in[L, U]$ for $t \in[-\tau, 0]$ for $i=0,1$, then $w_{i}(t) \in[L, U]$ for $t \geq 0$ for $i=0,1$. We anticipate that such a result will not hold unless certain conditions restrict the model parameters (including the birth function parameters), so it is also our intention to find such conditions if they are needed. 
To find the desired $L$ and $U$, we must first define two numbers, $M$ and $W$, according to whichever birth option is assumed to hold:

$$
\begin{gathered}
M= \begin{cases}\frac{1}{\lambda_{1}} & \text { if birth option (B1) holds } \\
\frac{2}{\alpha_{1}} & \text { if birth option (B2) holds } \\
\max \left\{\frac{1}{\lambda_{1}}, \frac{2}{\alpha_{1}}\right\} & \text { if birth option (B3) holds }\end{cases} \\
W= \begin{cases}\frac{2}{\lambda_{1}} & \text { if birth option (B1) holds } \\
\frac{3}{\alpha_{1}} & \text { if birth option (B2) holds } \\
\min \left\{\frac{2}{\lambda_{1}}, \frac{3}{\alpha_{1}}\right\} & \text { if birth option (B3) holds. }\end{cases}
\end{gathered}
$$

We have defined these numbers $M$ and $W$ because they satisfy several properties that will be important in establishing conditions for endemicity. We describe these properties in the next two lemmas.

Lemma 5.4. Let any one of the three birth options hold. Let $M$ and $W$ be defined according to the birth option that is assumed to hold. If birth option (B3) holds, then assume $\lambda_{1}<\alpha_{1}<$ $3 \lambda_{1}$. Then $M<W$, and $b_{0}(w)$ and $b_{1}(w)$ are monotonic decreasing for $w \in[M, W]$.

Proof. It is obvious that $M<W$ for birth options (B1) and (B2). For birth option (B3), we will have $M<W$ provided that $\frac{1}{\lambda_{1}}<\frac{3}{\alpha_{1}}$ and $\frac{2}{\alpha_{1}}<\frac{2}{\lambda_{1}}$ hold simultaneously, which is true if $\lambda_{1}<\alpha_{1}<3 \lambda_{1}$, which is true by assumption.

If birth option (B1) holds, then the birth functions are identical and equal $\lambda_{0} w e^{-\lambda_{1} w}$. But it is trivial to verify that this function is monotonic decreasing for $w \geq \frac{1}{\lambda_{1}}$, so it must be monotonic decreasing for $w \in[M, W]$ since, for birth option (B1), $M=\frac{1}{\lambda_{1}}$.

If birth option (B2) holds, then the birth functions are identical and equal $\alpha_{0} w^{2} e^{-\alpha_{1} w}$. But it is trivial to verify that this function is monotonic decreasing for $w \geq \frac{2}{\alpha_{1}}$, so it must be monotonic decreasing for $w \in[M, W]$ since, for birth option (B2), $M=\frac{2}{\alpha_{1}}$.

If birth option (B3) holds, we use the fact that $\lambda_{0} w e^{-\lambda_{1} w}$ is monotonic decreasing for $w \geq \frac{1}{\lambda_{1}}$, the fact that $\alpha_{0} w^{2} e^{-\alpha_{1} w}$ is monotonic decreasing for $w \geq \frac{2}{\alpha_{1}}$, and the definition of $M$ as the maximum of $\frac{1}{\lambda_{1}}$ and $\frac{2}{\alpha_{1}}$, to deduce that both the birth functions are monotonic decreasing for $w \in[M, W]$.

Lemma 5.5. Let any one of the three birth options hold. Let $M$ and $W$ be defined according to the birth option that is assumed to hold. If birth option (B3) holds, then assume $\lambda_{1}<\alpha_{1}<$ $3 \lambda_{1}$. Let $L$ and $U$ satisfy $M \leq L<U \leq W$.

(a) If birth options (B1) or (B2) hold, then the birth functions are identical, so we can define $b_{L}=b_{0}(L)=b_{1}(L)$ and $b_{U}=b_{0}(U)=b_{1}(U)$. Then $\frac{b_{L}}{U}<\frac{b_{U}}{L}$.

(b) If birth option (B3) holds, then there are values of the birth function parameters $\lambda_{0}, \lambda_{1}$, $\alpha_{0}, \alpha_{1}$ such that there exist $L$ and $U$ satisfying $b_{0}(L)=b_{1}(L)$ and $b_{0}(U)=b_{1}(U)$. When this happens define $b_{L}=b_{0}(L)=b_{1}(L)$ and $b_{U}=b_{0}(U)=b_{1}(U)$. Then $\frac{b_{L}}{U}<\frac{b_{U}}{L}$. 
Proof. Firstly we know by lemma 5.4 that $M<W$, so it is sensible to let $L$ and $U$ satisfy $M \leq L<U \leq W$.

Proof of (a). For birth option (B1), the birth functions are identical and equal $b(w)=\lambda_{0} w e^{-\lambda_{1} w}$. Define $h(w)=w b(w)=\lambda_{0} w^{2} e^{-\lambda_{1} w}$. Then

$$
\frac{d h(w)}{d w}=\lambda_{0} w e^{-\lambda_{1} w}\left(2-\lambda_{1} w\right) .
$$

It follows that $h(w)$ has a single turning point for $w>0$, and this occurs where $w=\frac{2}{\lambda_{1}}$. For $\frac{1}{\lambda_{1}}<w<\frac{2}{\lambda_{1}}$, it is clear that $h(w)$ is monotonic increasing. Hence if $M=\frac{1}{\lambda_{1}} \leq L<U \leq \frac{2}{\lambda_{1}}=W$, we must have $h(L)<h(U)$, which can be rewritten as $\frac{b(L)}{U}<\frac{b(U)}{L}$ since $h(w)=w b(w)$. Hence $\frac{b_{L}}{U}<\frac{b_{U}}{L}$.

For birth option (B2), the birth functions are identical and equal $b(w)=\alpha_{0} w^{2} e^{-\alpha_{1} w}$. Define $h(w)=w b(w)=\alpha_{0} w^{3} e^{-\alpha_{1} w}$. Then

$$
\frac{d h(w)}{d w}=\alpha_{0} w^{2} e^{-\alpha_{1} w}\left(3-\alpha_{1} w\right)
$$

It follows that $h(w)$ has a single turning point for $w>0$, and this occurs where $w=\frac{3}{\alpha_{1}}$. For $\frac{2}{\alpha_{1}}<w<\frac{3}{\alpha_{1}}$, it is clear that $h(w)$ is monotonic increasing. Hence if $M=\frac{2}{\alpha_{1}} \leq L<U \leq \frac{3}{\alpha_{1}}=W$, we must have $h(L)<h(U)$, which can be rewritten as $\frac{b(L)}{U}<\frac{b(U)}{L}$ since $h(w)=w b(w)$. Hence $\frac{b_{L}}{U}<\frac{b_{U}}{L}$.

Proof of (b). If there are values of the birth function parameters $\lambda_{0}, \lambda_{1}, \alpha_{0}, \alpha_{1}$ such that there exist $L$ and $U$ satisfying $M \leq L<U \leq W$ and $b_{0}(L)=b_{1}(L)$ and $b_{0}(U)=b_{1}(U)$, then the methods used to prove part (a) and the definitions of $M$ and $W$ for birth option (B3) quickly lead to the conclusion that $\frac{b_{L}}{U}<\frac{b_{U}}{L}$. We have found by inspection that suitable parameter values can exist. For example, if $\lambda_{0}=2.2, \lambda_{1}=3.1$, $\alpha_{0}=15.0$, and $\alpha_{1}=5.6$, then $M=0.3571, L=0.3684, U=0.4334$, and $W=0.5357$, which clearly gives $M<L<U<W$. See figure 5.1 for plots of the birth functions, showing the intersections at $L$ and $U$.

A complete understanding of the size and properties of the region of the $\lambda_{0}-\lambda_{1}-\alpha_{0}$ $\alpha_{1}$ parameter space such that appropriate $L$ and $U$ exist is still a matter for investigation.

Now we are in a position to prove that the creature can remain endemic on both patches:

Theorem 5.7. Let $w_{0}(t)$ and $w_{1}(t)$ satisfy (5.6) and (5.7), subject to (5.4). Suppose that both patches are identical, except perhaps the birth functions. Let any one of the three birth options -(B1), (B2), (B3) - hold. Let $M$ and $W$ be defined according to the birth option that is assumed to hold. If birth option (B3) holds, then assume $\lambda_{1}<\alpha_{1}<3 \lambda_{1}$ By lemma 5.4, $M<W$.

Suppose that $w_{i}(t) \in[L, U]$ for $t \in[-\tau, 0]$ for $i=0,1$, for $L$ and $U$ satisfying $M \leq L<$ $U \leq W$.

If birth option (B1) or (B2) hold then the birth functions are identical, so we may define $b_{L}=b_{0}(L)=b_{1}(L)$ and $b_{U}=b_{0}(U)=b_{1}(U)$ and we notice by lemma 5.5 that $\frac{b_{L}}{U}<\frac{b_{U}}{L}$. Trivially $b_{L}>b_{U}$. 


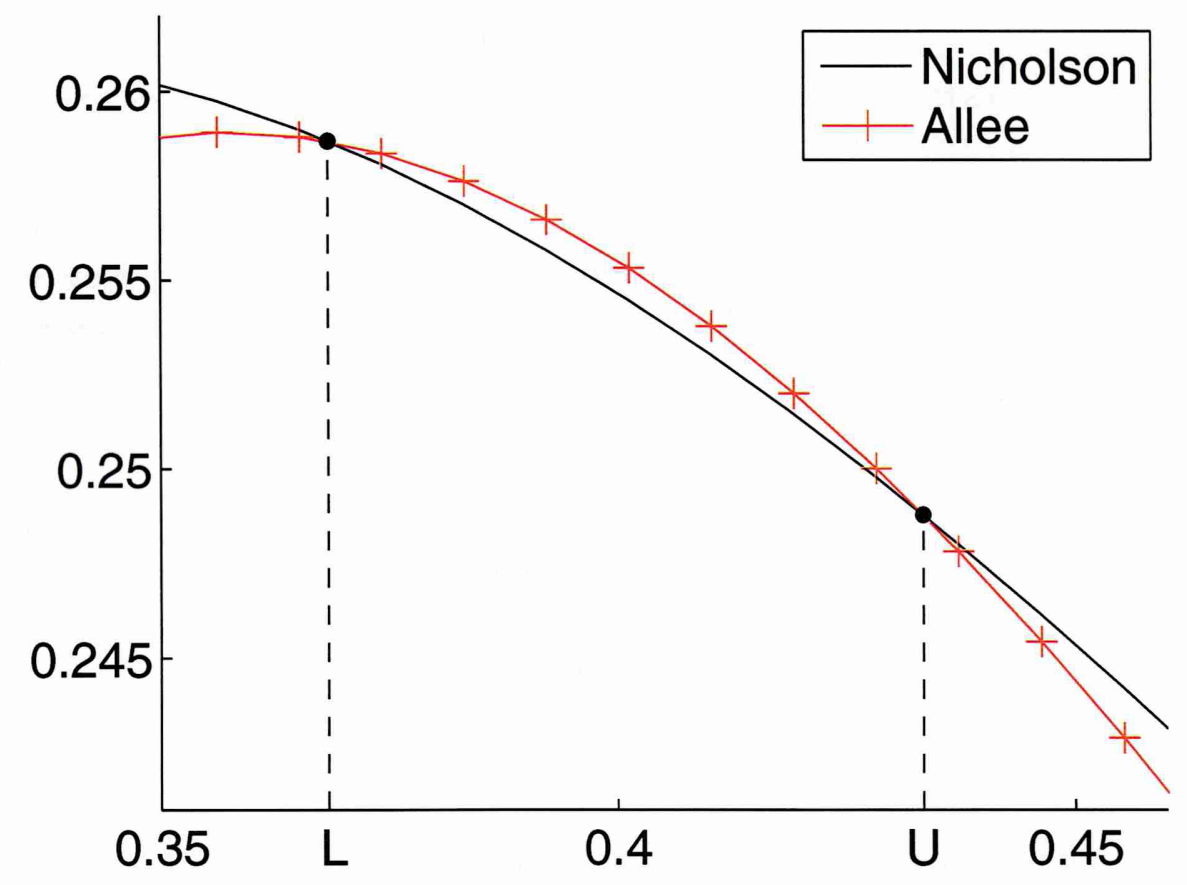

Figure 5.1: Plot of birth functions, namely $\lambda_{0} N e^{-\lambda_{1} N}$ (Nicholson) and $\alpha_{0} N^{2} e^{-\alpha_{1} N}$ (Allee), when $\lambda_{0}=2.2, \lambda_{1}=3.1, \alpha_{0}=15.0$, and $\alpha_{1}=5.6$. Horizontal axis is the independent variable $N$. Notice the two intersections at $N=L$ and $N=U$. 
If birth option (B3) holds then suppose that the birth function parameters are such that $L$ and $U$ exist satisfying $b_{0}(L)=b_{1}(L)$ and $b_{0}(U)=b_{1}(U)$. Define $b_{L}=b_{0}(L)=b_{1}(L)$ and $b_{U}=b_{0}(U)=b_{1}(U)$ and notice by lemma 5.5 that $\frac{b_{L}}{U}<\frac{b_{U}}{L}$. Trivially $b_{L}>b_{U}$.

It is possible to assume, then, for any of the three birth options that

$$
\frac{b_{L}}{U} \leq \frac{A-B}{C+D} \leq \frac{b_{U}}{L} .
$$

When (5.55) holds, then $w_{i}(t) \in[L, U]$ for $t \geq 0$ for $i=0,1$.

Before we give the proof, note that $\frac{A-B}{C+D}=\frac{d_{M}}{e^{*}}$, which is the mature death rate over the probability of surviving to maturity. Clearly $\frac{d_{M}}{e^{*}}$ is a measure of how harshly the environment treats the creature, since this quantity is higher if the mature death rate is higher or if the probability of surviving to maturity is lower. Thus the theorem, in a sense, asks for the environmental conditions to be intermediate (neither too harsh nor too forgiving), since $\frac{d_{M}}{e^{*}}$ is bounded above and below.

Proof. First observe by lemma 5.4 that $b_{0}(w)$ and $b_{1}(w)$ are monotonic decreasing for $w \in[L, U]$. But then, using the definitions of $b_{L}$ and $b_{U}$, we trivially know that $b_{L}>$ $b_{U}$. Since the initial data $w_{i}(t) \in[L, U]$ for $t \in[-\tau, 0]$ for $i=0,1$, it also follows immediately that

$$
b_{U} \leq b_{i}\left(w_{i}(t)\right) \leq b_{L} \text { for } t \in[-\tau, 0] \text { where } i=0,1 .
$$

Since the patches are identical (except possibly the birth functions), then (5.48) and (5.49) hold. But using (5.56) in equations (5.48) and (5.49) reveals that, for $t \in[0, \tau]$,

$$
\begin{aligned}
& \frac{d w_{0}(t)}{d t} \geq-A w_{0}(t)+B w_{1}(t)+(C+D) b_{U} \\
& \frac{d w_{1}(t)}{d t} \geq B w_{0}(t)-A w_{1}(t)+(C+D) b_{U} .
\end{aligned}
$$

It follows (theorem 1.1, pp. 78-79, [128]) that $w_{0}(t) \geq x(t)$ and $w_{1}(t) \geq y(t)$ for $t \in[0, \tau]$, where $x(0)=L \leq w_{0}(0)$ and $y(0)=L \leq w_{1}(0)$, and where, for $t \in[0, \tau]$, we have:

$$
\begin{aligned}
& \frac{d x(t)}{d t}=-A x(t)+B y(t)+(C+D) b_{U} \\
& \frac{d y(t)}{d t}=B x(t)-A y(t)+(C+D) b_{U} .
\end{aligned}
$$

This system in $x$ and $y$ has a unique fixed point $P_{1}=\left(X_{1}, Y_{1}\right)=\left(\frac{(C+D) b_{U}}{A-B}, \frac{(C+D) b_{U}}{A-B}\right)$. A phase portrait (see figure 5.2) reveals a trapping region $R_{1}$, bounded by the $x$ and $y$ axes and the nullclines. It is verified by routine calculations that the flow is inwards across all boundaries of $R_{1}$. The nullcline $\dot{x}=0$ can be written as $y=\frac{A}{B} x-\frac{(C+D) b_{U}}{B}$ and the nullcline $\dot{y}=0$ can be written as $y=\frac{B}{A} x+\frac{(C+D) b_{U}}{A}$ (bear in mind that $A>B$ ). It is clear that the line $y=x$ for $0<x<\frac{(C+D) b_{U}}{A-B}$ lies in the region $R_{1}$. So if the initial condition $(x(0), y(0))=(L, L)$ lies on this particular segment of the line $y=x$, then the trajectory $(x(t), y(t))$, determined by (5.59) and (5.60) for $t \in[0, \tau]$, will be confined to $R_{1}$. 


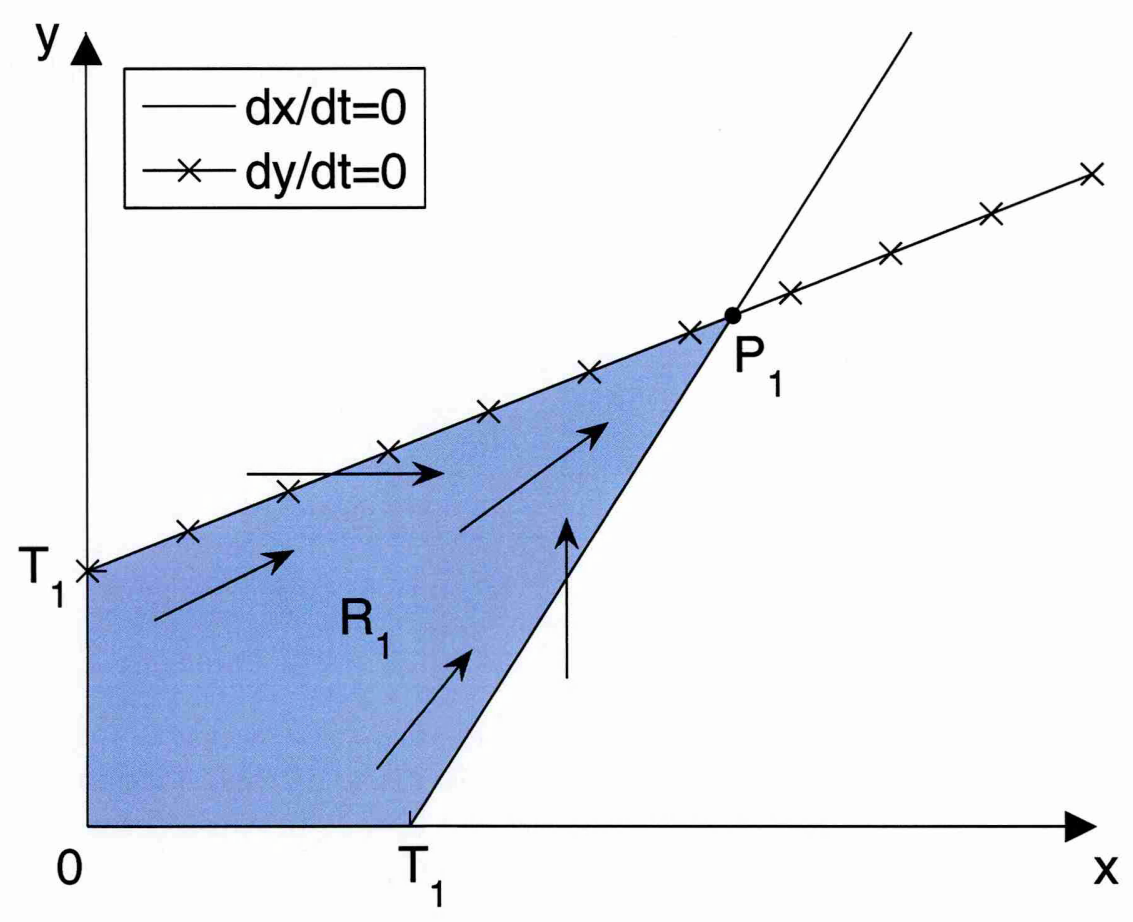

Figure 5.2: Phase portrait for the system given by (5.59) and (5.60). Here $T_{1}=\frac{(C+D) b_{U}}{A}$ is the $x$ and $y$ intercepts. Parameter choices: $A=2, B=1, C+D=0.5$. Birth option is (B1), with $\lambda_{0}=7.6, \lambda_{1}=1$. We have chosen $L=M=\frac{1}{\lambda_{1}}=1$ and $U=W=\frac{2}{\lambda_{1}}=2$. 
But (5.55) tells us that $\frac{A-B}{C+D} \leq \frac{b_{U}}{L}$, from which we see that $L<\frac{(C+D) b_{U}}{A-B}$. We also know that $L \geq M>0$. So $(x(0), y(0))=(L, L)$ must lie in $R_{1}$, and so $(x(t), y(t))$ must lie in $R_{1}$ for $t \in[0, \tau]$.

The region $R_{1}$ is more than just a trapping region. Inside it we have $\dot{x}>0$ and $\dot{y}>0$, so for the trajectory beginning at $(x(0), y(0))=(L, L)$, we must have $x(t) \geq L$ and $y(t) \geq L$ for $t \in[0, \tau]$. Consequently $w_{0}(t) \geq x(t) \geq L$ and $w_{1}(t) \geq y(t) \geq L$ for $t \in[0, \tau]$.

However, notice that the lower bounds for $w_{0}(t)$ and $w_{1}(t)$, namely $x(t)$ and $y(t)$, cannot increase beyond $P_{1}=\left(\frac{(C+D) b_{U}}{A-B}, \frac{(C+D) b_{U}}{A-B}\right)$. Using (5.55) and the fact that $b_{L}>b_{U}$, this means that $x(t)$ and $y(t)$ cannot increase beyond $U$. If this were not the case, this theorem would not necessarily hold.

Now observe that, using (5.56) in equations (5.48) and (5.49) reveals, for $t \in[0, \tau]$,

$$
\begin{aligned}
\frac{d w_{0}(t)}{d t} & \leq-A w_{0}(t)+B w_{1}(t)+(C+D) b_{L} \\
\frac{d w_{1}(t)}{d t} & \leq B w_{0}(t)-A w_{1}(t)+(C+D) b_{L} .
\end{aligned}
$$

It follows (theorem 1.1, pp. 78-79, [128]) that $w_{0}(t) \leq x(t)$ and $w_{1}(t) \leq y(t)$ for $t \in[0, \tau]$, where $x(0)=U \geq w_{0}(0)$ and $y(0)=U \geq w_{1}(0)$, and where, for $t \in[0, \tau]$, we have:

$$
\begin{aligned}
& \frac{d x(t)}{d t}=-A x(t)+B y(t)+(C+D) b_{L} \\
& \frac{d y(t)}{d t}=B x(t)-A y(t)+(C+D) b_{L} .
\end{aligned}
$$

This system in $x$ and $y$ has a unique fixed point $P_{2}=\left(X_{2}, Y_{2}\right)=\left(\frac{(C+D) b_{L}}{A-B}, \frac{(C+D) b_{L}}{A-B}\right)$. A phase portrait (see figure 5.3) reveals a trapping region $R_{2}$, bounded by the nullclines. Routine calculations verify that the flow is inwards across the boundaries of $R_{2}$. The nullcline $\dot{x}=0$ can be written as $y=\frac{A}{B} x-\frac{(C+D) b_{L}}{B}$ and the nullcline $\dot{y}=0$ can be written as $y=\frac{B}{A} x+\frac{(C+D) b_{L}}{A}$ (bear in mind that $A>B$ ). It is clear that the line $y=x$ for $x>\frac{(C+D) b_{L}}{A-B}$ lies in the region $R_{2}$. So if the initial condition $(x(0), y(0))=(U, U)$ lies on this particular segment of the line $y=x$, then the trajectory $(x(t), y(t))$, determined by (5.63) and (5.64) for $t \in[0, \tau]$, will be confined to $R_{2}$.

But (5.55) tells us that $\frac{b_{L}}{U} \leq \frac{A-B}{C+D}$, from which we see that $U>\frac{(C+D) b_{L}}{A-B}$. So $(x(0), y(0))=(U, U)$ must lie in $R_{2}$, and so $(x(t), y(t))$ must lie in $R_{2}$ for $t \in[0, \tau]$.

Inside $R_{2}$ we have $\dot{x}<0$ and $\dot{y}<0$, so for the trajectory beginning at $(x(0), y(0))=$ $(U, U)$, we must have $x(t) \leq U$ and $y(t) \leq U$ for $t \in[0, \tau]$. Consequently $w_{0}(t) \leq$ $x(t) \leq U$ and $w_{1}(t) \leq y(t) \leq U$ for $t \in[0, \tau]$.

However, notice that the upper bounds for $w_{0}(t)$ and $w_{1}(t)$, namely $x(t)$ and $y(t)$, cannot decrease below $P_{2}=\left(\frac{(C+D) b_{L}}{A-B}, \frac{(C+D) b_{L}}{A-B}\right)$. Using (5.55) and the fact that $b_{L}>b_{U}$, this means that $x(t)$ and $y(t)$ cannot decrease below $L$. If this were not the case, this theorem would not necessarily hold. 


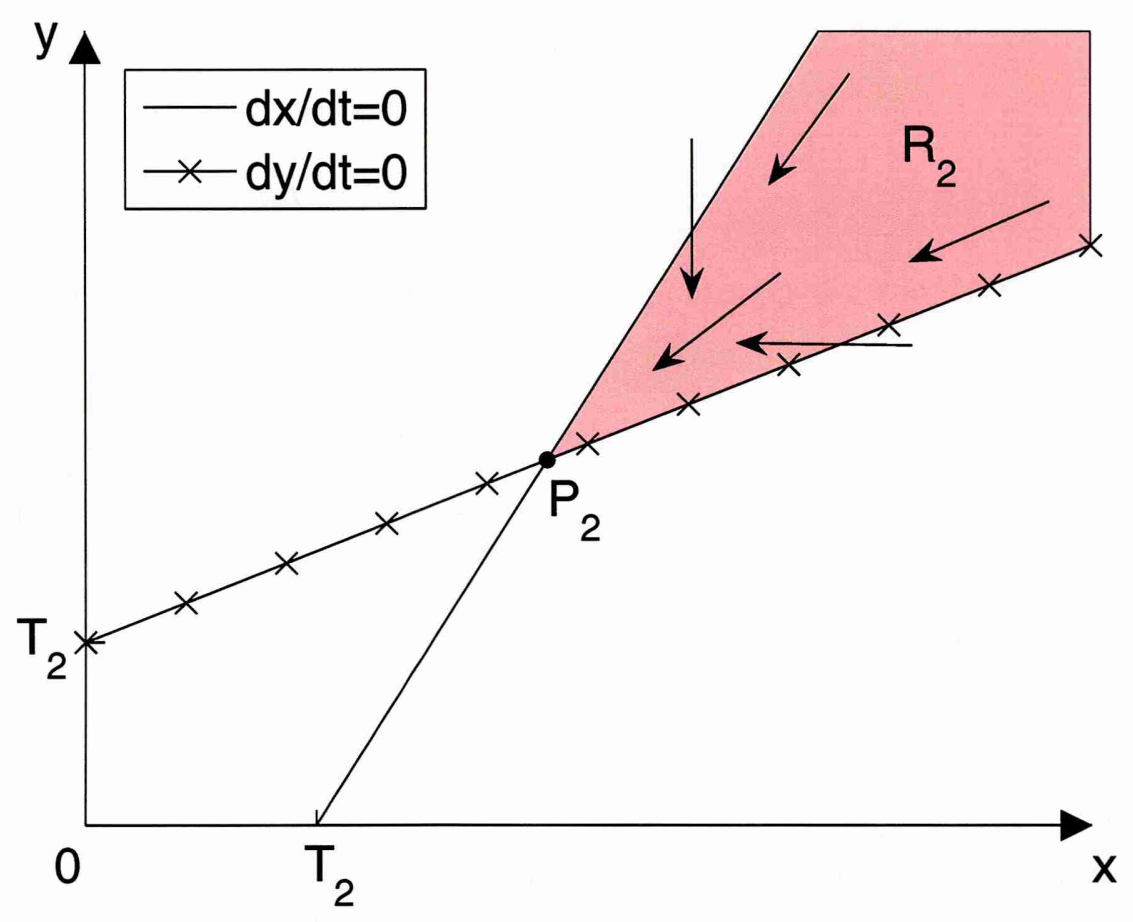

Figure 5.3: Phase portrait for the system given by (5.63) and (5.64). Here $T_{2}=\frac{(C+D) b_{L}}{A}$ is the $x$ and $y$ intercepts. Parameter choices: $A=2, B=1, C+D=0.5$. Birth option is (B1), with $\lambda_{0}=4, \lambda_{1}=1$. We have chosen $L=M=\frac{1}{\lambda_{1}}=1$ and $U=W=\frac{2}{\lambda_{1}}=2$. 


\begin{tabular}{|c|c|c|}
\hline Plot & Birth functions & $\begin{array}{c}\text { Initial data } \\
\left(w_{0}(t), w_{1}(t)\right) \equiv(U, V) \\
\text { for } t \in[-\tau, 0]\end{array}$ \\
\hline Top left & $b_{0}(w)=b_{1}(w)=0.5 w$ & $(U, V)=(100,100)$ \\
\hline Top middle & $b_{0}(w)=b_{1}(w)=0.5 w e^{-w}$ & $(U, V)=(100,100)$ \\
\hline Top right & $b_{0}(w)=b_{1}(w)=0.5 w^{2} e^{-w}$ & $(U, V)=(100,100)$ \\
\hline Bottom left & same as top left & $(U, V)=(10,20)$, \\
& & $(20,30),(40,30)$, \\
& & $(40,10),(25,5)$ \\
\hline Bottom middle & same as top middle & same as bottom left \\
\hline Bottom right & same as top right & same as bottom left \\
\hline
\end{tabular}

Table 5.1: Parameter values for plots in figure 5.4

Thus, we have proven, for $i=0,1$, that if $w_{i}(t) \in[L, U]$ for $t \in[-\tau, 0]$, then $w_{i}(t) \in$ $[L, U]$ for $t \in[0, \tau]$. A methods of steps induction (induction on the subintervals $[j \tau,(j+1) \tau]$ for $j \geq 0)$ will immediately then tell us that $w_{i}(t) \in[L, U]$ for $t \geq 0$.

Notice how theorem 5.7, an endemicity result on two patches, generalises an earlier endemicity result for a species inhabiting a single patch with Allee births, namely theorem 3.12. By setting migration rates to zero in theorem 5.7, it is possible to recover theorem 3.12 as well as to deduce an endemicity result on a single patch when there are Nicholson births.

\subsection{Simulations}

In figure 5.4 we include simulations for the model of (5.6), (5.7), and (5.4) demonstrating extinction. For each simulation, results in section 5.3 guarantee extinction on both patches. In the first row of figure 5.4 the plots illustrate the populations on both patches changing over time in three cases: both birth functions linear (left plot); both birth functions Nicholson (middle); both birth functions Allee (right). The second row of figure 5.4 gives phase portrait solutions for a range of initial data for the same three cases. Notice that the usual rules for two-dimensional phase portraits do not necessarily hold when the system contains a delay. However, for visual clarity, we have plotted only trajectories that do not intersect.

In all the plots in figure 5.4 , we set

$$
\tau=1, A=1.3, B=2, E=1, F=2.4, C=D=G=H=0.2 .
$$

The birth functions and initial conditions used to produce the simulations in figure 5.4 are given in table 5.1.

Figure 5.5 is laid out in the same way as figure 5.4, except that now the plots demonstrate endemicity, with endemicity guaranteed in each plot by results in section 5.6. The point $P$ in the middle and right plots of the second row of figure 5.5 is a fixed point, which the simulations suggest to be stable. 

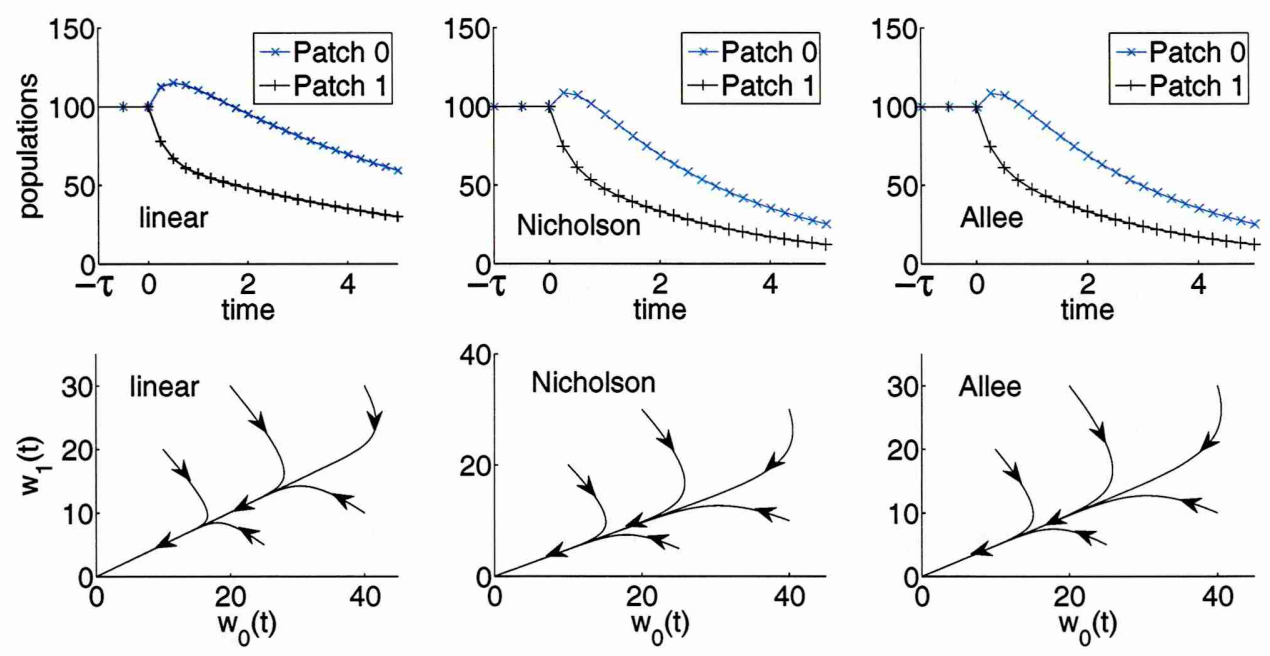

Figure 5.4: Extinction simulations. See section 5.7 and table 5.1 for a description.

The birth functions and initial conditions used to produce the simulations in figure 5.5 are given in table 5.2.

In the top left and bottom left plots in figure 5.5, we set

$$
\tau=1, A=1.3, B=2, E=1, F=2.4, C=D=G=H=0.2 .
$$

In all other plots in figure 5.5, we set

$$
\tau=1, B=E=1, C=D=G=H=0.2 .
$$

In all plots except top left and bottom left, the birth function on patch 0 is the same as the birth function on patch 1 . Therefore in each case there is no ambiguity in defining $A$ (as we have done in each case) by the following formula (chosen so that condition (5.55) will hold, so that theorem 5.7 will apply):

$$
A=B+\left(\frac{C+D}{2}\right)\left(\frac{b(L)}{U}+\frac{b(U)}{L}\right),
$$

where $b(\cdot)=b_{0}(\cdot)=b_{1}(\cdot)$ is the birth function appropriate to each case, as defined in table 5.2.

\subsection{Discussion}

We have examined a model for a creature which has a life cycle with two stages and which inhabits two patches. Our examination has involved three different choices of birth function, namely linear, Nicholson, and Allee. We have discovered conditions on the model parameters such that extinction will occur on both patches. When the birth function on both patches is of Allee type, we have shown that there is always a population level beneath which extinction becomes inevitable on both patches. We have also found conditions on the parameters, and additionally in some cases the 


\begin{tabular}{|c|c|c|}
\hline Plot & Birth functions & $\begin{array}{c}\text { Initial data } \\
\left(w_{0}(t), w_{1}(t)\right) \equiv(U, V) \\
\text { for } t \in[-\tau, 0]\end{array}$ \\
\hline Top left & $b_{0}(w)=7.15 w, b_{1}(w)=13.2 w$ & $(U, V)=(100,100)$ \\
\hline Top middle & $b_{0}(w)=b_{1}(w)=0.1 w e^{-0.05 w}$ & $(U, V)=(10,20)$ \\
\hline Top right & $b_{0}(w)=b_{1}(w)=0.1 w^{2} e^{-w}$ & $(U, V)=(20,30)$ \\
\hline Bottom left & same as top left & $(U, V)=(20,100)$, \\
& & $(20,20),(120,20)$, \\
& & $(180,20),(20,150)$ \\
\hline Bottom middle & same as top middle & $(U, V)=(10,10)$, \\
& & $(11,20),(20,11)$, \\
& & $(20,20),(15,20)$, \\
& & $(10,15),(15,10)$, \\
& & $(15,15),(20,15)$ \\
\hline Bottom right & same as top right & $(U, V)=(20,20)$, \\
& & $(21,30),(30,21)$, \\
& & $(30,30),(20,25)$, \\
& & $(25,20),(30,25)$, \\
\hline
\end{tabular}

Table 5.2: Parameter values for plots in figure 5.5
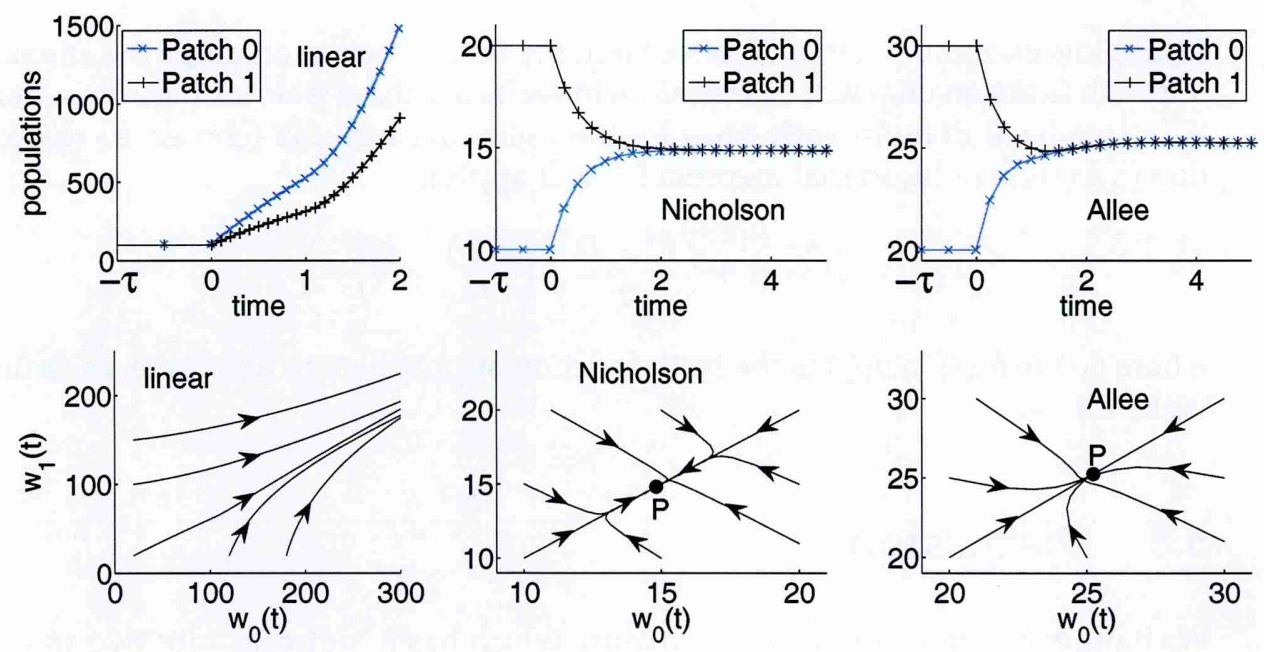

Figure 5.5: Endemicity simulations. See section 5.7 and table 5.2 for a description. 
initial data, such that the creature will remain endemic on both patches. Simulations have corroborated our results.

The results in this chapter have a practical value, for if the creature being modelled is a pest, it is in our interests to know when it will die out naturally and when it may persist. After all, if it will die out naturally, there may be no need to implement a pest control stratagem. Thus, knowing when a pest will die out naturally can save us the effort, time, and environmental consequences of applying pesticides. By contrast, knowing that a pest will persist can enable us to form a response, which may be a particular control stratagem. In the next chapter, we construct successful control stratagems for the two-patch model.

In this chapter we have extended the local stability analysis of So, Wu, and $\mathrm{Zou}$ [129]. However, a complete understanding of the global behaviour of the model, even for the birth functions considered in this chapter, has not yet been attained. Ultimately we would wish to have a complete understanding of global behaviour when only a few general assumptions are made about the birth functions. There is scope for future work. 


\title{
Chapter 6
}

\section{Culling of a structured population on two patches}

\begin{abstract}
"Animals have these advantages over man: they never hear the clock strike, they die without any idea of death, they have no theologians to instruct them, their last moments are not disturbed by unwelcome and unpleasant ceremonies, their funerals cost them nothing, and no one starts lawsuits over their wills."
\end{abstract}

Voltaire (1694 - 1778)

\subsection{Introduction}

In this chapter we continue our analysis of the model of chapter 5 for a creature with a life cycle consisting of two stages (immature and adult) which inhabits two patches, which we label patch 0 and patch 1 . We mentioned in the introduction in chapter 5 that the creature could be a pest. After all, many pests lead stage-structured life cycles and are driven, by instincts to eat and mate, to move from one region to another. Thus the patches could represent neighbouring farms suffering from a common pest infestation or regions sharing both a border and a pest. For the rest of this chapter we shall assume that the creature is a pest. Our purpose will be to find adult impulsive culling regimes to control it on both patches.

We invite the reader to reacquaint themself with the modelling assumptions of section 5.2. We also remind ourselves of the model of chapter 5 by repeating equations (5.4), (5.5), (5.6), and (5.7):

$$
\begin{gathered}
w_{i}(t)=\phi_{i}(t) \geq 0 \text { for } t \in[-\tau, 0], i=0,1, \\
b_{0}(0)=0, \quad b_{1}(0)=0, \quad \text { and, for } w>0, \quad b_{0}(w)>0, \quad b_{1}(w)>0,
\end{gathered}
$$

and, for $t>0$,

$$
\begin{aligned}
& \frac{d w_{0}(t)}{d t}=-A w_{0}(t)+B w_{1}(t)+C b_{0}\left(w_{0}(t-\tau)\right)+D b_{1}\left(w_{1}(t-\tau)\right) \\
& \frac{d w_{1}(t)}{d t}=E w_{0}(t)-F w_{1}(t)+G b_{0}\left(w_{0}(t-\tau)\right)+H b_{1}\left(w_{1}(t-\tau)\right) .
\end{aligned}
$$


Here $w_{i}(t)$ is the adult population on patch $i$ at time $t, \phi_{i}(t)$ is a function of initial and finite data, and $b_{i}(\cdot)$ is the birth function on patch $i(i=0,1)$. Also $A, B, C, D, E, F, G, H$ are positive constants with $A>E$ and $F>B$. The biological meanings of the model parameters are described in section 5.2. As in chapter 5, we will suppose that either birth function, $b_{0}$ or $b_{1}$, can be of linear, Nicholson, or Allee type (see subsection 3.2.1 for a description of these functions and notice that these functions satisfy (6.2)). The term "population" will be used as a shorthand for "adult population" in the rest of this chapter.

We saw in chapter 5 (section 5.6) that the pest can remain endemic on both patches, where $b_{0}$ and $b_{1}$ can be any of the three birth function types under consideration and need not be the same as each other, if certain conditions hold on the model parameters and additionally in some cases on the initial data. Then, if the pest remains endemic in sufficiently high numbers and is causing enough damage (in terms of crop destruction or disease-spreading), control may be warranted. Of course the pest may only be a problem on one of the patches but we will see in the next section that implementing a control strategy on only one of the patches may not necessarily eradicate the pest on that patch. Hence there may be situations in which culling is warranted on both patches simultaneously, so we construct simultaneous culling regimes in sections 6.3, 6.4, and 6.5. In section 6.6 we explore these simultaneous regimes, in particular showing that the regimes will succeed provided that consecutive culls on each patch occur sufficiently close together in time. In section 6.7 we show that if both birth functions are of Allee type, then only a finite number of culls is needed on both patches in order for the pest to be eradicated on both patches. Simulations corroborating our results are included in section 6.8 and we end the chapter with a discussion in section 6.9.

\subsection{Culling on one patch}

Suppose that the appropriate authorities on patch 0 decide that the pest be controlled on this patch by the introduction of an adult impulsive culling regime. Their decision is independent of the authorities on patch 1 . No culling is carried out on patch 1 . (The situation where culling is implemented on patch 1 only may be analysed in exactly the same way as if on patch 0 only, which is why we consider only the latter case.) Let the culling regime on patch 0 satisfy the following definition:

Definition 6.1. Culls occur on patch 0 at times $t_{i}, i \geq 1$, where $0<t_{1}<t_{2}<t_{3}<\ldots$ and $t_{i} \rightarrow \infty$ as $i \rightarrow \infty$. The cull at time $t_{i}$ is the $i$-th cull. At the $i$-th cull the population on patch 0 is impulsively rescaled thus: $w_{0}\left(t_{i}\right)=\epsilon_{i} w_{0}\left(t_{i}^{-}\right)$where $0<\epsilon_{i}<1$ and $t_{i}^{-}$is the time "momentarily" before $t_{i}$. Also at time $t_{i}$ we assume $w_{1}\left(t_{i}\right)=w_{1}\left(t_{i}^{-}\right)$. When $t \neq t_{i}$, then $w_{0}(t)$ and $w_{1}(t)$ evolve according to equations (6.1), (6.2), (6.3), and (6.4). A culling regime is regular if $\epsilon_{i}=\epsilon^{*}$ and $t_{i+1}-t_{i}=T$ for $i \geq 1$ for constants $\epsilon^{*}, T$ with $0<\epsilon^{*}<1$ and $T>0$. A regime is successful if $w_{0}(t) \rightarrow 0$ as $t \rightarrow \infty$.

It is trivial to adapt lemma 5.1 to see that positivity will still hold in the model of (6.1), (6.2), (6.3), and (6.4) when culls are imposed on patch 0 as outlined in definition 6.1. In particular strict positivity will hold if $w_{0}(0)>0$ and $w_{1}(0)>0$.

We have seen in chapter 3 (subsections 3.4.1, 3.4.2, and 3.4.3) that, for a pest living on a single patch, there will exist a successful regular culling regime whether the birth function is linear, Nicholson, or Allee. The next two theorems show that a successful regular culling regime need not exist on patch 0 if the birth function on patch 1 is Nicholson, Allee, or linear. 
Theorem 6.1. Make the assumptions in definition 6.1 and assume $w_{0}(0)>0$ and $w_{1}(0)>0$. Suppose the birth function on patch 1 , namely $b_{1}$, is Nicholson or Allee. Also suppose that if $N(t)>0$ for $t \in[-\tau, 0]$ and $\frac{d N(t)}{d t}=H b_{1}(N(t-\tau))-F N(t)$ for $t>0$, then $H, F$, and the parameters defining $b_{1}$ are such that $K_{1}<N(t)<K_{2}$ for positive constants $K_{1}, K_{2}$ and for all $t$ large enough. (Conditions on $H, F$, and the parameters defining $b_{1}$ appropriate to this last assumption may be found in theorems 3.7, 3.8, and 3.9 for $b_{1}$ Nicholson and in theorem 3.12 for $b_{1}$ Allee.) Then a successful regular culling regime does not exist on patch 0 .

Proof. Suppose for a contradiction that a successful regular culling regime (with parameters $\epsilon^{*}, T$ ) does exist on patch 0 . Then $w_{0}(t) \rightarrow 0$ as $t \rightarrow \infty$. But then by (6.4), $\frac{d w_{1}(t)}{d t} \rightarrow H b_{1}\left(w_{1}(t-\tau)\right)-F w_{1}(t)$ as $t \rightarrow \infty$. Hence (by section 1, [110]) $w_{1}(t) \rightarrow N(t)$ as $t \rightarrow \infty$ where $\frac{d N(t)}{d t}=H b_{1}(N(t-\tau))-F N(t)$ for $t>0$ and where $N(t)=w_{1}(t)>0$ for $t \in[-\tau, 0]$. But, by assumption, $K_{1}<N(t)<K_{2}$ for $t$ large enough, so since $w_{1}(t) \rightarrow N(t)$ we must have $\frac{K_{1}}{2}<w_{1}(t)<\frac{3 K_{2}}{2}$ for $t$ large enough, say $t>t^{*}$. Then, since $b_{1}$ is Nicholson or Allee, there exist positive constants $M_{1}$ and $M_{2}$ such that $M_{1} \leq b_{1}\left(w_{1}(t)\right) \leq M_{2}$ for $t>t^{*}$. In particular, we may deduce by (6.3) and strict positivity of $w_{0}(t)$ and $w_{1}(t)$ (see the comment immediately after definition 6.1), that, for $t>t^{*}+\tau$, except at times where culls are performed,

$$
\frac{d w_{0}(t)}{d t}>K-A w_{0}(t)
$$

where $K=D M_{1}+\frac{B K_{1}}{2}$. It follows that $w_{0}(t) \geq N_{1}(t)$ for $t \geq t_{i}$ where $t_{i}$ is the time of the first cull occurring after $t^{*}+\tau$, where $N_{1}\left(t_{i}\right)=w_{0}\left(t_{i}\right)>0$, where $N_{1}\left(t_{j}\right)=$ $\epsilon^{*} N_{1}\left(t_{j}^{-}\right)$for $j>i$, and where

$$
\frac{d N_{1}(t)}{d t}=K-A N_{1}(t) \text { for } t>t_{i}, t \neq t_{j} \text { for } j>i .
$$

It is easy to see that $N_{1}(t)>0$ for $t \geq t_{i}$. Solving for $N_{1}(t)$ on $\left[t_{j}, t_{j+1}\right)$ for any $j>i$ gives

$$
N_{1}(t)=\frac{K}{A}+\left(N_{1}\left(t_{j}\right)-\frac{K}{A}\right) e^{-A\left(t-t_{j}\right)} .
$$

Then:

$$
\begin{aligned}
N_{1}\left(t_{j+1}\right) & =\epsilon^{*} N_{1}\left(t_{j+1}^{-}\right)=\epsilon^{*}\left[\frac{K}{A}+\left(N_{1}\left(t_{j}\right)-\frac{K}{A}\right) e^{-A T}\right] \\
& >\frac{\epsilon^{*} K}{A}\left(1-e^{-A T}\right) \text { (since } N_{1}\left(t_{j}\right)>0 \text { by strict positivity). }
\end{aligned}
$$

Hence, for $j>i+1$, we have $N_{1}\left(t_{j}\right)>\frac{\epsilon^{*} K}{A}\left(1-e^{-A T}\right)$. Also, by (6.7), we can write, for $t \in\left[t_{j}, t_{j+1}\right)$ for $j>i+1$, that:

$$
\begin{aligned}
N_{1}(t) & \geq \min \left(N_{1}\left(t_{j}\right), \frac{K}{A}\right) \\
& \geq \min \left(\frac{\epsilon^{*} K}{A}\left(1-e^{-A T}\right), \frac{K}{A}\right) \\
& =\frac{\epsilon^{*} K}{A}\left(1-e^{-A T}\right) .
\end{aligned}
$$


Thus $w_{0}(t)>N_{1}(t)>\frac{\epsilon^{*} K}{A}\left(1-e^{-A T}\right)>0$ for $t \geq t_{i+2}$ where $i$ is a finite positive integer. But clearly this cannot be if $w_{0}(t) \rightarrow 0$ as $t \rightarrow \infty$. This contradiction shows that a successful regular culling regime does not exist on patch 0 .

From this last theorem we see that, when $b_{1}$ is either a Nicholson or Allee birth function, then culling will sometimes be needed on both patches even if we only require the pest to be eradicated on one of the patches. What happens if $b_{1}$ is a linear birth function? Although it is unrealistic to expect a population to grow indefinitely, the following theorem may have some short-term validity and shows that, once again, culling may be needed on both patches even if we only seek to control the pest on one of the patches.

Theorem 6.2. Make the assumptions in definition 6.1. Suppose further that the initial data is strictly positive:

$$
w_{i}(t) \geq M_{i} \text { for } t \in[-\tau, 0], i=0,1,
$$

for positive constants $M_{0}, M_{1}$. Assume the birth function $b_{1}$ is linear, with $b_{1}(N)=\Lambda_{1} N$, and suppose $H \Lambda_{1}>F$. In addition to the culling assumptions in definition 6.1, let $\underline{\epsilon}=$ $\inf _{i \geq 1} \epsilon_{i}$ and suppose $\underline{\epsilon}>0$. Also suppose that $t_{i+1}-t_{i} \geq \Delta>0$ for $i \geq 1$. Then $w_{0}(t) \rightarrow \infty$ and $w_{1}(t) \rightarrow \infty$ as $t \rightarrow \infty$.

Proof. We know that between culls, that is, for $t \in\left[t_{i}, t_{i+1}\right), i \geq 1$, then

$$
\begin{aligned}
& \frac{d w_{0}(t)}{d t}=-A w_{0}(t)+B w_{1}(t)+C b_{0}\left(w_{0}(t-\tau)\right)+D \Lambda_{1} w_{1}(t-\tau) \\
& \frac{d w_{1}(t)}{d t}=E w_{0}(t)-F w_{1}(t)+G b_{0}\left(w_{0}(t-\tau)\right)+H \Lambda_{1} w_{1}(t-\tau) .
\end{aligned}
$$

Culls occur only on patch 0 , so we have $w_{0}\left(t_{i}\right)=\epsilon_{i} w_{0}\left(t_{i}^{-}\right)$and $w_{1}\left(t_{i}\right)=w_{1}\left(t_{i}^{-}\right)$for $i \geq 1$. Applying theorem 1.1 from pages 78-79 of [128] individually on the time intervals $\left[-\tau, t_{1}\right)$ and $\left[t_{i}, t_{i+1}\right)$ for $i \geq 1$, and using positivity of $w_{0}(t)$ and $w_{1}(t)$ and (6.2), we may deduce that, for $t \geq-\tau$, we have $w_{0}(t) \geq x_{0}(t)$ and $w_{1}(t) \geq x_{1}(t)$ where:

$$
\begin{gathered}
x_{0}(t)=M_{0} \leq w_{0}(t) \text { and } x_{1}(t)=M_{1} \leq w_{1}(t) \text { for } t \in[-\tau, 0], \\
x_{0}\left(t_{i}\right)=\epsilon_{i} x_{0}\left(t_{i}^{-}\right) \text {and } x_{1}\left(t_{i}\right)=x_{1}\left(t_{i}^{-}\right) \text {for } i \geq 1,
\end{gathered}
$$

and where, for $t \in\left[0, t_{1}\right)$ and $t \in\left[t_{i}, t_{i+1}\right)$ for $i \geq 1$, we have:

$$
\begin{aligned}
& \frac{d x_{0}(t)}{d t}=B x_{1}(t)-A x_{0}(t) \\
& \frac{d x_{1}(t)}{d t}=-F x_{1}(t)+H \Lambda_{1} x_{1}(t-\tau) .
\end{aligned}
$$

Notice that $x_{0}(t)$ and $x_{1}(t)$ satisfy positivity for $t \geq-\tau$. Using the fact that $x_{1}\left(t_{i}\right)=$ $x_{1}\left(t_{i}^{-}\right)$for $i \geq 1$ as well as the fact that (6.16) is valid on $\left[0, t_{1}\right)$ and $\left[t_{i}, t_{i+1}\right)$ for $i \geq 1$, we deduce that, for $t \geq 0$,

$$
\frac{d x_{1}(t)}{d t}=-F x_{1}(t)+H \Lambda_{1} x_{1}(t-\tau) .
$$


By the initial data for $x_{1}(t)$ (see (6.13)), and by (6.17) and the assumption that $H \Lambda_{1}>F$, we may deduce by theorem 3.3 that

$$
x_{1}(t) \rightarrow \infty \text { as } t \rightarrow \infty \text {. }
$$

It follows that $w_{1}(t) \geq x_{1}(t) \rightarrow \infty$ as $t \rightarrow \infty$.

It remains for us to prove that $w_{0}(t) \rightarrow \infty$. This will be true if, for any $K>0$, there exists $T>0$ such that, for all $t \geq T$, then $w_{0}(t) \geq K$.

So, pick any $K>0$. Since $x_{1}(t) \rightarrow \infty$ as $t \rightarrow \infty$, then, for any $M>0$, there exists $T_{M}>0$ such that, for all $t \geq T_{M}$, then $x_{1}(t) \geq M$. In particular this will hold when

$$
M=\max \left\{\frac{A K}{B}, \frac{A K}{\underline{\epsilon} B\left(1-e^{-A \Delta}\right)}\right\} .
$$

For the rest of this proof, assume that (6.19) holds.

Now $t_{i} \rightarrow \infty$ as $i \rightarrow \infty$, so there exists $i^{*} \geq 1$ such that, for all $i \geq i^{*}$, then $t_{i} \geq T_{M}$. Hence, by (6.15), we find that

$$
\frac{d x_{0}(t)}{d t} \geq B M-A x_{0}(t) \text { for } t \geq t_{i^{*}}, t \neq t_{i} \text { for } i \geq i^{*} .
$$

It follows (see theorem 1.1, pp. 78-79, [128]) that if $i \geq i^{*}$, then $x_{0}(t) \geq y(t)$ for $t \in\left[t_{i}, t_{i+1}\right)$, where $y\left(t_{i}\right)=x_{0}\left(t_{i}\right)$ and, for $t \in\left[t_{i}, t_{i+1}\right)$,

$$
\frac{d y(t)}{d t}=B M-A y(t) \text {. }
$$

Solving for $y(t)$ reveals that, for $t \in\left[t_{i}, t_{i+1}\right)$, we have

$$
x_{0}(t) \geq y(t)=\frac{B M}{A}+e^{-A\left(t-t_{i}\right)}\left(x_{0}\left(t_{i}\right)-\frac{B M}{A}\right) .
$$

Next we show that

$$
x_{0}\left(t_{i}\right) \geq K \text { for all } i \geq i^{*}+1 .
$$

To this end, pick any $i \geq i^{*}+1$. For such an $i$ we can write, by (6.22),

$$
\begin{aligned}
x_{0}\left(t_{i}^{-}\right) & \geq \frac{B M}{A}+e^{-A\left(t_{i}^{-}-t_{i-1}\right)}\left(x_{0}\left(t_{i-1}\right)-\frac{B M}{A}\right) \\
& =\frac{B M}{A}\left(1-e^{-A\left(t_{i}^{-}-t_{i-1}\right)}\right)+x_{0}\left(t_{i-1}\right) e^{-A\left(t_{i}^{-}-t_{i-1}\right)} .
\end{aligned}
$$

Since $x_{0}(t)$ satisfies positivity we know that $x_{0}\left(t_{i-1}\right) \geq 0$. Also we know by assumption that $t_{i}-t_{i-1} \geq \Delta$ for $i \geq 2$. Then we deduce by (6.24) that

$$
x_{0}\left(t_{i}^{-}\right) \geq \frac{B M}{A}\left(1-e^{-A \Delta}\right) .
$$

But by (6.19) we have $M \geq \frac{A K}{\underline{\epsilon} B\left(1-e^{-A \Delta}\right)}$, so that $\frac{B M}{A}\left(1-e^{-A \Delta}\right) \geq \frac{K}{\epsilon}$. Then by (6.25) we have $x_{0}\left(t_{i}^{-}\right) \geq \frac{K}{\underline{\epsilon}}$. Hence:

$$
x_{0}\left(t_{i}\right)=\epsilon_{i} x_{0}\left(t_{i}^{-}\right) \geq \underline{\epsilon} x_{0}\left(t_{i}^{-}\right) \geq \underline{\epsilon}\left(\frac{K}{\underline{\epsilon}}\right)=K,
$$


as required.

By (6.23) and (6.22), we have, for $t \in\left[t_{i}, t_{i+1}\right), i \geq i^{*}+1$, that

$$
x_{0}(t) \geq \frac{B M}{A}+e^{-A\left(t-t_{i}\right)}\left(K-\frac{B M}{A}\right) .
$$

By (6.19), we have $M \geq \frac{A K}{B}$, so that $K-\frac{B M}{A} \leq 0$. But then the right hand side of (6.27) is monotonic increasing for $t \in\left[t_{i}, t_{i+1}\right)$, so it must be bounded below by its value at $t=t_{i}$, which is $K$. Hence $x_{0}(t) \geq K$ for $t \in\left[t_{i}, t_{i+1}\right)$ for any $i \geq i^{*}+1$. But then $w_{0}(t) \geq x_{0}(t) \geq K$ for $t \geq T=t_{i^{*}+1}$. Thus for any $K>0$, we have found a $T>0$ such that $w_{0}(t) \geq K$ for all $t \geq T$. Therefore $w_{0}(t) \rightarrow \infty$ as $t \rightarrow \infty$.

\subsection{Culling regimes}

We have seen in the last section that there are circumstances under which culling on both patches may be warranted. It is decided that a culling program will be implemented to eradicate the pest on both patches. Those people responsible for each patch agree to co-operate in the sense that they will both introduce culling programs. In order to make the co-operation flexible enough to stand a chance of succeeding, it is agreed that the culling regimes of the different patches will be independent. It will not matter which starts first, nor, when both regimes have begun, should the times or strengths of the culls in the one patch depend on the other.

Of course the regimes cannot be completely without rules, and it is the purpose of this chapter to discover a set of rules that ensure pest eradication without impeding the flexibility just mentioned. At this stage we must introduce some terminology and lay down some fundamental rules for our regimes.

Definition 6.2. Assume (6.1) and (6.2) hold. For $k=0,1$, do the following:

Define as regime $k$ the culling regime for patch $k$. Let the culls on patch $k$ occur at times $t_{i}^{(k)}$, for $i \geq 1$, where $0<t_{1}^{(k)}<t_{2}^{(k)}<t_{3}^{(k)} \ldots$ and $t_{i}^{(k)} \rightarrow \infty$ as $i \rightarrow \infty$.

Also suppose

$$
t_{i+1}^{(k)}-t_{i}^{(k)} \leq \tau
$$

for $i \geq 1$.

Further, let the $i$-th cull on patch $k$ (which occurs at time $t_{i}^{(k)}$ ) scale the population on patch $k$ impulsively by a factor of $\epsilon_{i}^{(k)}$ where $0<\epsilon_{i}^{(k)}<1$ for all $i \geq 1$. Thus $w_{k}\left(t_{i}^{(k)}\right)=$ $\epsilon_{i}^{(k)} w_{k}\left(t_{i}^{(k)}-\right)$ where $t_{i}^{(k)}-$ denotes the time "momentarily" before time $t_{i}^{(k)}$. At times when culls do not occur, assume that the populations evolve according to (6.3) and (6.4).

Define $\epsilon_{(k)}^{*}=\sup _{i \geq 1} \epsilon_{i}^{(k)}$ and assume $\epsilon_{(k)}^{*}<1$.

The regimes are successful if together they ensure that both $w_{0}(t) \rightarrow 0$ and $w_{1}(t) \rightarrow 0$ as $t \rightarrow \infty$.

Observe that no restriction is made on when the first cull occurs on either patch after the collection of initial data. This is to give us, in a real-world scenario, sufficient time to analyse the initial data before making an appropriate control decision. 
Next we define a number which will prove important in simplifying our later analysis:

Definition 6.3. If $t_{1}^{(0)}=t_{1}^{(1)}$, then the regimes begin simultaneously. This is highly unlikely, given the independence of the regimes. But if this is the case, define $j=0$.

If $t_{1}^{(0)}<t_{1}^{(1)}$, define $j=1$.

Finally, if $t_{1}^{(1)}<t_{1}^{(0)}$, define $j=0$.

It should be clear that the earliest starting culling regime is on patch $1-j$. In other words, patch $1-j$ is the patch on which the very first cull occurs of any of the culls of either regime. It follows that patch $j$ is the patch on which the later starting regime is carried out.

We stress that $j$ is to be considered fixed henceforth, with the knowledge that it could be fixed as either 0 or 1 .

We must now find conditions on the times and strengths of the culls specific enough to be workable. The following upper bound lemma will allow us to create a more powerful upper bound lemma, which will in turn suggest how to construct our culling regimes.

Lemma 6.1. Assume (6.1) and (6.2) hold. Let $K_{0}, K_{1}$ be positive constants. Assume $w_{i}(t) \leq$ $K_{i}$ for $t \in[a-\tau, a]$ for $i=0,1$. Also assume $w_{i}(a) \leq \epsilon_{i} K_{i}$ where $0<\epsilon_{i} \leq 1$ and $i=$ 0,1 . Assume further that $w_{0}(t)$ satisfies (6.3) and $w_{1}(t)$ satisfies (6.4) on $[a, a+\tau]$. Finally assume that the birth functions can be bounded above by linear functions: $b_{0}\left(w_{0}\right) \leq \Lambda_{0} w_{0}$ and $b_{1}\left(w_{1}\right) \leq \Lambda_{1} w_{1}$ where $\Lambda_{0}>0$ and $\Lambda_{1}>0$.

Then $w_{i}(t) \leq x_{i}(t)$ for $t \in[a, b]$ where $a<b \leq a+\tau$ and where

$$
\left(\begin{array}{c}
x_{0}(t) \\
x_{1}(t)
\end{array}\right)=\frac{1}{B \rho} \chi_{0} e^{\lambda_{0}(t-a)}\left(\begin{array}{c}
B \\
A+\lambda_{0}
\end{array}\right)+\frac{1}{B \rho} \chi_{1} e^{\lambda_{1}(t-a)}\left(\begin{array}{c}
B \\
A+\lambda_{1}
\end{array}\right)+\left(\begin{array}{c}
M_{0} \\
M_{1}
\end{array}\right)
$$

where

$$
\begin{gathered}
\lambda_{0}=\frac{-(A+F)+\rho}{2}<0, \\
\lambda_{1}=\frac{-(A+F)-\rho}{2}<0, \\
\rho=\sqrt{ }\left((A+F)^{2}-4(A F-B E)\right)>0, \\
M_{0}=\frac{F L_{0}+B L_{1}}{A F-B E}>0, \\
M_{1}=\frac{E L_{0}+A L_{1}}{A F-B E}>0, \\
L_{0}=C \Lambda_{0} K_{0}+D \Lambda_{1} K_{1}>0, \quad L_{1}=G \Lambda_{0} K_{0}+H \Lambda_{1} K_{1}>0, \\
\chi_{0}=B\left(\epsilon_{1} K_{1}-M_{1}\right)-\left(A+\lambda_{1}\right)\left(\epsilon_{0} K_{0}-M_{0}\right),
\end{gathered}
$$

and

$$
\chi_{1}=\left(A+\lambda_{0}\right)\left(\epsilon_{0} K_{0}-M_{0}\right)-B\left(\epsilon_{1} K_{1}-M_{1}\right)
$$


Proof. We begin by noting that, since $w_{i}(t) \leq K_{i}$ for $t \in[a-\tau, a]$ for $i=0,1$, then (using theorem 1.1, pp. 78-79, [128] and positivity) we have $w_{i}(t) \leq x_{i}(t)$ for $t \in[a, b]$ where $a<b \leq a+\tau$ for $x_{i}(t)$ satisfying

$$
\begin{gathered}
x_{i}(t)=K_{i} \geq w_{i}(t) \text { on }[a-\tau, a], \\
x_{i}(a)=\epsilon_{i} K_{i} \geq w_{i}(a),
\end{gathered}
$$

and

$$
\begin{aligned}
& \frac{d x_{0}(t)}{d t}=-A x_{0}(t)+B x_{1}(t)+C \Lambda_{0} K_{0}+D \Lambda_{1} K_{1} \\
& \frac{d x_{1}(t)}{d t}=E x_{0}(t)-F x_{1}(t)+G \Lambda_{0} K_{0}+H \Lambda_{1} K_{1},
\end{aligned}
$$

for $t \in[a, a+\tau]$.

It is straightforward to find $x_{i}(t)$ on $[a, a+\tau]$ for $i=0,1$ since this amounts to solving an initial value problem (with initial conditions (6.39)) for a linear system (equations (6.40) and (6.41)). Instead of going through the lengthy process of finding the solution we invite the reader to substitute in the solution already given in (6.29).

It is important to know that $\rho>0$, that $\lambda_{1}<\lambda_{0}<0$, that $A+\lambda_{1}<0$, and that $A+\lambda_{0}>0$. All of these results and the other inequalities stated in (6.30) to (6.35) follow from the fact that $A>E$ and $F>B$, as well the knowledge that $A, B, C, D, E, F, G, H, \Lambda_{0}, \Lambda_{1}, K_{1}, K_{2}$ are all strictly positive.

We prove here that $\rho>0$ and that $A+\lambda_{0}>0$ and leave the reader to verify the other inequalities. We deduce that $\rho>0$ and, indeed, real, by noting that:

$$
(A+F)^{2}-4(A F-B E)=(A-F)^{2}+4 B E>0 .
$$

And we see that $A+\lambda_{0}>0$ as follows:

$$
\begin{aligned}
A+\lambda_{0} & =\frac{(A-F)+\rho}{2} \\
& =\frac{(A-F)+\sqrt{ }\left((A-F)^{2}+4 B E\right)}{2} \quad \text { (using (6.42)) } \\
& >\frac{(A-F)+\sqrt{ }\left((A-F)^{2}\right)}{2} \\
& \geq 0 .
\end{aligned}
$$

Our final lemma will suggest our culling regimes. Once we have chosen our culling regimes, we will have to prove that they successfully eradicate the pest on both patches.

Lemma 6.2. For $x_{i}(t)$ given in (6.29), we have

$$
x_{i}(t) \leq K\left(\epsilon_{i} e^{\lambda_{1}(t-a)}+\left(\theta_{1}+\theta_{2}\right)\left(1-e^{\lambda_{1}(t-a)}\right)\right),
$$

for $i=0,1$, where

$$
K=\max \left(K_{0}, K_{1}\right)>0,
$$


and

$$
\begin{gathered}
\theta_{1}=\max \left(\frac{F\left(C \Lambda_{0}+D \Lambda_{1}\right)+B\left(G \Lambda_{0}+H \Lambda_{1}\right)}{A F-B E}, \frac{E\left(C \Lambda_{0}+D \Lambda_{1}\right)+A\left(G \Lambda_{0}+H \Lambda_{1}\right)}{A F-B E}\right), \\
\theta_{2}=\left(\frac{1}{\rho}\right) \max \left(B-\left(A+\lambda_{1}\right),\left(A+\lambda_{0}\right)\left(\frac{1}{B}\right)\left(B-\left(A+\lambda_{1}\right)\right)\right) .
\end{gathered}
$$

Note that $\theta_{1}$ and $\theta_{2}$ are both positive.

Proof. First we establish (6.43) for $x_{0}(t)$.

We begin by noting that, by (6.29) and using the relationship $\lambda_{0}=\rho+\lambda_{1}$, we can write

$$
\begin{aligned}
x_{0}(t)= & \left(\frac{1}{\rho}\right) e^{\lambda_{0}(t-a)}\left[B\left(\epsilon_{1} K_{1}-M_{1}\right)-\left(A+\lambda_{1}\right)\left(\epsilon_{0} K_{0}-M_{0}\right)\right] \\
& +\left(\frac{1}{\rho}\right) e^{\lambda_{1}(t-a)}\left[\left(A+\lambda_{0}\right)\left(\epsilon_{0} K_{0}-M_{0}\right)-B\left(\epsilon_{1} K_{1}-M_{1}\right)\right]+M_{0} \\
= & \epsilon_{0} K_{0} e^{\lambda_{1}(t-a)}+M_{0}\left(1-e^{\lambda_{1}(t-a)}\right) \\
& +\left(\frac{1}{\rho}\right)\left(e^{\lambda_{0}(t-a)}-e^{\lambda_{1}(t-a)}\right) \\
& \times\left[B\left(\epsilon_{1} K_{1}-M_{1}\right)-\epsilon_{0} K_{0}\left(A+\lambda_{1}\right)+M_{0}\left(A+\lambda_{1}\right)\right] .
\end{aligned}
$$

Now $\lambda_{1}<\lambda_{0}$ and $t-a \geq 0$ for $t \in[a, a+\tau]$ and $\rho>0$. So

$$
\left(\frac{1}{\rho}\right)\left(e^{\lambda_{0}(t-a)}-e^{\lambda_{1}(t-a)}\right) \geq 0 .
$$

Also $-B M_{1}<0$ and $M_{0}\left(A+\lambda_{1}\right)<0$ since $B, M_{1}, M_{0}$ are positive and $A+\lambda_{1}$ is negative. Hence, from (6.47), we see that

$$
\begin{aligned}
x_{0}(t) \leq & \epsilon_{0} K_{0} e^{\lambda_{1}(t-a)}+M_{0}\left(1-e^{\lambda_{1}(t-a)}\right) \\
& +\left(\frac{1}{\rho}\right)\left(e^{\lambda_{0}(t-a)}-e^{\lambda_{1}(t-a)}\right)\left[B \epsilon_{1} K_{1}-\epsilon_{0} K_{0}\left(A+\lambda_{1}\right)\right] .
\end{aligned}
$$

Next, observe that $e^{\lambda_{0}(t-a)} \leq 1$ since $\lambda_{0}<0$ and $t-a \geq 0$. Therefore $e^{\lambda_{0}(t-a)}-$ $e^{\lambda_{1}(t-a)} \leq 1-e^{\lambda_{1}(t-a)}$. Also, $0<\epsilon_{0} K_{0} \leq \epsilon_{0} K$. But then we can write

$$
x_{0}(t) \leq \epsilon_{0} K e^{\lambda_{1}(t-a)}+\left(M_{0}+\Omega_{0}\right)\left(1-e^{\lambda_{1}(t-a)}\right),
$$

where

$$
\Omega_{0}=\left(\frac{1}{\rho}\right)\left[B \epsilon_{1} K_{1}-\epsilon_{0} K_{0}\left(A+\lambda_{1}\right)\right]
$$

Observe that $0<\epsilon_{0}, \epsilon_{1} \leq 1$ and $A+\lambda_{1}<0$ and $K_{0}, K_{1} \leq K$. Then it is clear that

$$
\Omega_{0} \leq K\left(\frac{1}{\rho}\right)\left[B-\left(A+\lambda_{1}\right)\right] \leq K \theta_{2} .
$$


Also, by (6.33) and (6.35), and the fact that $K_{0}, K_{1}<K$, we find

$$
\begin{aligned}
M_{0} & =\frac{F L_{0}+B L_{1}}{A F-B E}=\frac{F\left(C \Lambda_{0} K_{0}+D \Lambda_{1} K_{1}\right)+B\left(G \Lambda_{0} K_{0}+H \Lambda_{1} K_{1}\right)}{A F-B E} \\
& \leq K\left(\frac{F\left(C \Lambda_{0}+D \Lambda_{1}\right)+B\left(G \Lambda_{0}+H \Lambda_{1}\right)}{A F-B E}\right) \leq K \theta_{1} .
\end{aligned}
$$

Introducing inequalities (6.52) and (6.53) into (6.50) yields (6.43) for $x_{0}(t)$, as required.

The second part of the proof is to establish (6.43) for $x_{1}(t)$.

The method is similar to the method used to establish (6.43) for $x_{0}(t)$, but the details are fiddly enough to be worth giving explicitly.

We begin by noting that, by (6.29) and the fact that $\lambda_{1}=\lambda_{0}-\rho$, we can write

$$
\begin{aligned}
x_{1}(t)= & \left(\frac{1}{B \rho}\right)\left(A+\lambda_{0}\right) e^{\lambda_{0}(t-a)}\left[B\left(\epsilon_{1} K_{1}-M_{1}\right)-\left(A+\lambda_{1}\right)\left(\epsilon_{0} K_{0}-M_{0}\right)\right] \\
& +M_{1}+\left(\frac{1}{B \rho}\right)\left(A+\lambda_{1}\right) e^{\lambda_{1}(t-a)} \\
& \quad \times\left[\left(A+\lambda_{0}\right)\left(\epsilon_{0} K_{0}-M_{0}\right)-B\left(\epsilon_{1} K_{1}-M_{1}\right)\right] \\
= & \epsilon_{1} K_{1} e^{\lambda_{1}(t-a)}+M_{1}\left(1-e^{\lambda_{1}(t-a)}\right) \\
& +\left(\frac{1}{B \rho}\right)\left(e^{\lambda_{0}(t-a)}-e^{\lambda_{1}(t-a)}\right) \zeta
\end{aligned}
$$

where

$$
\zeta=\left[B\left(\epsilon_{1} K_{1}-M_{1}\right)\left(A+\lambda_{0}\right)-\left(\epsilon_{0} K_{0}-M_{0}\right)\left(A+\lambda_{0}\right)\left(A+\lambda_{1}\right)\right] .
$$

Recall equation (6.48) and notice that $-B M_{1}\left(A+\lambda_{0}\right)<0$ and that $M_{0}\left(A+\lambda_{0}\right)(A+$ $\left.\lambda_{1}\right)<0$, allowing us to write

$$
\begin{aligned}
& x_{1}(t) \leq \epsilon_{1} K_{1} e^{\lambda_{1}(t-a)}+M_{1}\left(1-e^{\lambda_{1}(t-a)}\right) \\
&+\left(\frac{1}{B \rho}\right)\left(e^{\lambda_{0}(t-a)}-e^{\lambda_{1}(t-a)}\right) \\
& \times\left[B \epsilon_{1} K_{1}\left(A+\lambda_{0}\right)-\epsilon_{0} K_{0}\left(A+\lambda_{0}\right)\left(A+\lambda_{1}\right)\right] .
\end{aligned}
$$

As above, we have $e^{\lambda_{0}(t-a)}-e^{\lambda_{1}(t-a)} \leq 1-e^{\lambda_{1}(t-a)}$. Also $0<\epsilon_{1} K_{1} \leq \epsilon_{1} K$. We may thus write

$$
x_{1}(t) \leq \epsilon_{1} K e^{\lambda_{1}(t-a)}+\left(M_{1}+\Omega_{1}\right)\left(1-e^{\lambda_{1}(t-a)}\right),
$$

where

$$
\Omega_{1}=\left(A+\lambda_{0}\right)\left(\frac{1}{B \rho}\right)\left[B \epsilon_{1} K_{1}-\epsilon_{0} K_{0}\left(A+\lambda_{1}\right)\right] .
$$

Observe that $0<\epsilon_{0}, \epsilon_{1} \leq 1$ and $A+\lambda_{1}<0$. Also $A+\lambda_{0}>0$ and $K_{0}, K_{1} \leq K$. Then it is clear that

$$
\Omega_{1} \leq K\left(A+\lambda_{0}\right)\left(\frac{1}{B \rho}\right)\left(B-\left(A+\lambda_{1}\right)\right) \leq K \theta_{2} .
$$


Also, by (6.34) and (6.35), and the fact that $K_{0}, K_{1}<K$, we find

$$
\begin{aligned}
M_{1} & =\frac{E L_{0}+A L_{1}}{A F-B E}=\frac{E\left(C \Lambda_{0} K_{0}+D \Lambda_{1} K_{1}\right)+A\left(G \Lambda_{0} K_{0}+H \Lambda_{1} K_{1}\right)}{A F-B E} \\
& \leq K\left(\frac{E\left(C \Lambda_{0}+D \Lambda_{1}\right)+A\left(G \Lambda_{0}+H \Lambda_{1}\right)}{A F-B E}\right) \leq K \theta_{1} .
\end{aligned}
$$

Introducing inequalities (6.59) and (6.60) into (6.57) yields (6.43) for $x_{1}(t)$, as required.

The proof is complete.

We summarise the critical aspects of lemmas 6.1 and 6.2 in a self-evident corollary:

Corollary 6.1. Assume $w_{i}(t) \leq K_{i}$ for $t \in[a-\tau, a]$ for $i=0,1$ where $K_{0}$ and $K_{1}$ are positive constants and let $K=\max \left(K_{0}, K_{1}\right)$. Also assume $w_{i}(a) \leq \epsilon_{i} K_{i}$ where $0<\epsilon_{i} \leq 1$ and $i=0,1$. Assume further that $w_{0}(t)$ satisfies (6.3) and $w_{1}(t)$ satisfies (6.4) on $[a, b]$ where $a<b \leq a+\tau$. Assume also that (6.2) holds and that the birth functions can be bounded above by linear functions: $b_{0}\left(w_{0}\right) \leq \Lambda_{0} w_{0}$ and $b_{1}\left(w_{1}\right) \leq \Lambda_{1} w_{1}$ where $\Lambda_{0}$ and $\Lambda_{1}$ are positive constants. Suppose that no culls occur on either patch for $t \in(a, b)$.

Recall that $\lambda_{1}$ is a negative constant (equation (6.31)) and define the positive constant $\theta_{3}$ as follows:

$$
\theta_{3}=\theta_{1}+\theta_{2},
$$

with $\theta_{1}$ given by (6.45) and $\theta_{2}$ given by (6.46). Note that $\theta_{3}$ does not depend on $K_{i}$ or $\epsilon_{i}$.

Then, for $t \in[a, b]$ for $i=0,1$, we have

$$
w_{i}(t) \leq K\left(\epsilon_{i} e^{\lambda_{1}(t-a)}+\theta_{3}\left(1-e^{\lambda_{1}(t-a)}\right)\right) .
$$

From corollary 6.1 we will deduce, via lemma 6.3 , lemma 6.4 , and theorem 6.3 below, that if $\theta_{3}<1$ then the populations will go extinct on both patches in the absence of culling in such a way that both populations can be bounded above by decreasing sequences. We have of course proven that extinction can occur naturally on both patches in theorem 5.2 although the condition for extinction is rather simpler than $\theta_{3}<1$ (note that $\theta_{3}$ is a somewhat large expression). Establishing extinction when $\theta_{3}<1$ will tell us that culling is unnecessary when $\theta_{3}<1$. Also, knowing that culling will not be needed unless $\theta_{3} \geq 1$ will guide us in defining appropriate culling regimes in section 6.4.

Lemma 6.3. Assume $w_{i}(t) \leq U_{i}$ for $t \in[m \tau,(m+1) \tau]$ for $i=0,1$, where $U_{0}, U_{1}$ are positive constants and $m$ is some integer $\geq-1$. Let $U=\max \left(U_{0}, U_{1}\right)$. Also assume that (6.3) and (6.4) hold for $t \geq(m+1) \tau$. Suppose that the birth functions satisfy (6.2) and can be bounded above by linear functions: $b_{0}\left(w_{0}\right) \leq \Lambda_{0} w_{0}$ and $b_{1}\left(w_{1}\right) \leq \Lambda_{1} w_{1}$ where $\Lambda_{0}$ and $\Lambda_{1}$ are positive constants. Let $\theta_{3}=\theta_{1}+\theta_{2}$ with $\theta_{1}$ given by (6.45) and $\theta_{2}$ given by (6.46). Suppose $\theta_{3}<1$. Then, for $t \geq(m+1) \tau$, we have:

$$
w_{i}(t) \leq U\left(e^{\lambda_{1}(t-(m+1) \tau)}+\theta_{3}\left(1-e^{\lambda_{1}(t-(m+1) \tau)}\right)\right)=U\left(\theta_{3}+e^{\lambda_{1}(t-(m+1) \tau)}\left(1-\theta_{3}\right)\right) .
$$


Proof. The result will be true if we can show inductively on $k \geq m+1$ that (6.63) holds for $t \in[k \tau,(k+1) \tau]$. The basis step (for $k=m+1$ ) is immediately true by combining our assumptions with corollary 6.1. In particular, notice that since no culls occurs when $t=(m+1) \tau$, we can let $\epsilon_{0}=\epsilon_{1}=1$ in applying corollary 6.1.

To establish an inductive step, assume that (6.63) holds on $k \tau,(k+1) \tau$ for some $k \geq m+1$. Then, since $\theta_{3}<1$ and $\lambda_{1}<0$, it is easy to see that $w_{i}(t) \leq U$ for $t \in k \tau,(k+1) \tau$. It is also evident that

$$
w_{i}((k+1) \tau) \leq U\left(e^{\lambda_{1}((k+1) \tau-(m+1) \tau)}+\theta_{3}\left(1-e^{\lambda_{1}((k+1) \tau-(m+1) \tau)}\right)\right) .
$$

Notice that $0<e^{\lambda_{1}((k+1) \tau-(m+1) \tau)}+\theta_{3}\left(1-e^{\lambda_{1}((k+1) \tau-(m+1) \tau)}\right)<1$ since $0<\theta_{3}<$ 1 and $\lambda_{1}<0$. We may thus apply corollary 6.1 with $a=(k+1) \tau, b=(k+2) \tau$, $\epsilon_{i}=e^{\lambda_{1}((k+1) \tau-(m+1) \tau)}+\theta_{3}\left(1-e^{\lambda_{1}((k+1) \tau-(m+1) \tau)}\right)($ for $i=0,1)$, and $K_{i}=U$ (for $i=0,1)$, to find that, for $t \in[(k+1) \tau,(k+2) \tau]$ :

$$
\begin{aligned}
w_{i}(t) \leq & U\left(\epsilon_{i} e^{\lambda_{1}(t-(k+1) \tau)}+\theta_{3}\left(1-e^{\lambda_{1}(t-(k+1) \tau)}\right)\right) \\
\leq & U\left[\left(e^{\lambda_{1}((k+1) \tau-(m+1) \tau)}+\theta_{3}\left(\not 1-e^{\lambda_{1}((k+1) \tau-(m+1) \tau)}\right)\right) e^{\lambda_{1}(t-(k+1) \tau)}\right. \\
& \left.\quad+\theta_{3}\left(1-e^{\lambda_{1}(t-(k+1) \tau)}\right)\right] \\
= & U\left(e^{\lambda_{1}(t-(m+1) \tau)}+\theta_{3}\left(1-e^{\lambda_{1}(t-(m+1) \tau)}\right)\right)
\end{aligned}
$$

By (6.65), the inductive step is established.

Lemma 6.4. Make the assumptions in lemma 6.3. Then, for $i=0,1$, we have

$$
w_{i}(t) \leq \frac{1}{2}\left(1+\theta_{3}\right) U \text { for } t \in[k \tau,(k+1) \tau] \text { for all } k \geq k^{*},
$$

where $k^{*}=(m+2)+\left\lfloor\frac{\ln 2}{-\lambda_{1} \tau}\right\rfloor$ where $\rfloor$ denotes the greatest integer function.

Proof. An argument very similar to the type of argument used in the proof of lemma 3.2 will establish lemma 6.4 .

Theorem 6.3. Assume (as initial data) that $w_{i}(t) \leq U_{i}$ for $t \in[-\tau, 0]$ for $i=0,1$. Let $U=\max \left(U_{0}, U_{1}\right)$. Also assume that (6.3) and (6.4) hold for $t \geq 0$. Suppose the birth functions satisfy (6.2) and can be bounded above by linear functions: $b_{0}\left(w_{0}\right) \leq \Lambda_{0} w_{0}$ and $b_{1}\left(w_{1}\right) \leq \Lambda_{1} w_{1}$. Let $\theta_{3}$ be defined as in lemma 6.3. Suppose $\theta_{3}<1$. Then:

$$
w_{i}(t) \leq \gamma^{n} U \text { for } t \in[k \tau,(k+1) \tau] \text { for all } k \geq k_{n},
$$

where $\gamma=\frac{1}{2}\left(1+\theta_{3}\right)<1, k_{n}=(\Omega+1)+(n-1)(\Omega+2)$, and $\Omega=\left\lfloor\frac{\ln 2}{-\lambda_{1} \tau}\right\rfloor$.

Hence $w_{i}(t) \rightarrow 0$ as $t \rightarrow \infty$ for $i=0,1$.

Proof. An argument closely resembling the type of argument used in the proof of theorem 3.1 (but using lemma 6.4 instead of lemma 3.2) will establish theorem 6.3. 
Assuming as in theorem 6.3 that the birth functions can be bounded above by linear functions, theorem 5.2 establishes extinction on both patches when

$$
\max \left((C+G) \Lambda_{0},(D+H) \Lambda_{1}\right)<\min (A-E, F-B),
$$

whereas theorem 6.3 establishes extinction when $\theta_{3}<1$ where $\theta_{3}$ is a fairly large expression. It would be pleasing to know if one of these conditions implies the other. We leave this as an exercise for the reader - the matter is not of great importance to the remainder of this thesis.

\subsection{Definition of our culling regimes}

In this section the purpose of the calculations of the previous section shall become clear.

If we make the assumptions in corollary 6.1 , then in particular we assume, for positive constants $a$ and $K$, that $w_{i}(t) \leq K$ for $t \in[a-\tau, a]$. Moreover we deduce by corollary 6.1 that:

$$
w_{i}(t) \leq K\left(\epsilon_{i} e^{\lambda_{1}(t-a)}+\theta_{3}\left(1-e^{\lambda_{1}(t-a)}\right)\right) \text { for } t \in[a, b],
$$

where $b$ is a constant satisfying $a<b \leq a+\tau$. Suppose we let $\delta$ be a constant satisfying $0<\delta<1$ and suppose further that

$$
\epsilon_{i} e^{\lambda_{1}(t-a)}+\theta_{3}\left(1-e^{\lambda_{1}(t-a)}\right) \leq \delta \text { for } t \in[a, b]
$$

Then by the assumptions in corollary 6.1 and combining (6.68) with (6.69), we find, for $i=0,1$, that:

$$
w_{i}(t) \leq K \text { for } t \in[a-\tau, a]
$$

and

$$
w_{i}(t) \leq \delta K \text { for } t \in[a, b] \text { and where } \delta<1 .
$$

But this is exactly the kind of situation we are looking for. The upper bounds for the populations decrease from one time interval to the next. If the culling regimes on the two patches allow this pattern of decreasing upper bounds to continue indefinitely, then the populations will go extinct.

Now we have seen by theorem 6.3 that culling is not needed unless $\theta_{3} \geq 1$. But if $\theta_{3} \geq 1$, then

$$
\epsilon_{i} e^{\lambda_{1}(t-a)}+\theta_{3}\left(1-e^{\lambda_{1}(t-a)}\right)=\theta_{3}+e^{\lambda_{1}(t-a)}\left(\epsilon_{i}-\theta_{3}\right)
$$

is monotonic non-decreasing for $t>a$ when $0<\epsilon_{i} \leq 1$ and $\lambda_{1}<0$. In particular, then, if the function in (6.72) is defined on a time interval $t \in[a, b]$, it is bounded above on this interval by its value at $t=b$. Therefore if its value at $t=b$ is bounded above by $\delta<1$, then the function will be bounded above by $\delta<1$ on the interval $t \in[a, b]$.

The upshot of the remarks made so far in this section is that if we make the assumptions in corollary 6.1 , assume also that $\theta_{3} \geq 1$, and suppose for a constant $\delta$ (with $0<\delta<1$ ) that

$$
\epsilon_{i} e^{\lambda_{1}(b-a)}+\theta_{3}\left(1-e^{\lambda_{1}(b-a)}\right)<\delta,
$$


then $w_{i}(t) \leq K$ for $t \in[a-\tau, a]$ and $w_{i}(t) \leq \delta K<K$ for $t \in[a, b]$ where $a<$ $b \leq a+\tau$. Such reasoning suggests how to define a condition on the culling regimes that will ensure eradication on both patches. In fact, recalling the definition of $j$ (see definition 6.3), recalling also the notation given to the culling regimes in definition 6.2, and bearing in mind that culling is not needed unless $\theta_{3} \geq 1$, we are led to define the following quantities:

$$
\begin{aligned}
\delta_{1-j} & =\sup _{i \geq 1}\left\{\epsilon_{i}^{(1-j)} e^{\lambda_{1}\left(t_{i+1}^{(1-j)}-t_{i}^{(1-j)}\right)}+\theta_{3}\left(1-e^{\lambda_{1}\left(t_{i+1}^{(1-j)}-t_{i}^{(1-j)}\right)}\right)\right\} \\
\delta_{j} & =\sup _{i \geq 1}\left\{\epsilon_{i}^{(j)} e^{\lambda_{1}\left(t_{i+1}^{(j)}-t_{i}^{(j)}\right)}+\theta_{3}\left(1-e^{\lambda_{1}\left(t_{i+1}^{(j)}-t_{i}^{(j)}\right)}\right)\right\} \\
\delta & =\max \left\{\delta_{1-j}, \delta_{j}\right\} .
\end{aligned}
$$

It now seems sensible to conjecture that (when $\theta_{3} \geq 1$ ) eradication will occur if the culling regimes satisfy, in addition to the conditions given in definition 6.2 , the following condition:

$$
\delta<1 \text { where } \delta \text { is given by (6.76). }
$$

We will turn this conjecture into a theorem in the next section (section 6.5).

The significance of (6.77) will be explored in section 6.6. Suffice it to say at this stage that (6.77) will clearly hold when $\theta_{3} \geq 1$ if the inter-cull times on both patches are small enough.

\subsection{A proof that our regimes are successful}

We have become acquainted with the positive constant $\theta_{3}$ defined in corollary 6.1 , in particular noticing by theorem 6.3 that, if $\theta_{3}<1$, then the pest dies out on both patches in the absence of culling in such a way that both populations can be bounded above by decreasing sequences. Then, if $\theta_{3}<1$, the pest populations will not flare up sufficiently to ruin crops or cause an epidemic before dying out, so culling is not necessary. In this section we prove (in theorem 6.4) that, when $\theta_{3} \geq 1$, then the culling regimes satisfying definition 6.2 and condition (6.77) successfully eradicate the pest on both patches. For the remainder of this section assume that $\theta_{3} \geq 1$ and that the culling regimes satisfy definition 6.2 and condition (6.77).

Before stating and proving theorem 6.4 , we must recall the definition of the fixed number $j$ in definition 6.3. We repeat here that patch $1-j$ is the patch on which the very first cull occurs of any of the culls of either regime and that patch $j$ is the patch on which the later starting regime is carried out.

Also before theorem 6.4, we must define two subsequences which will be of importance to us:

Definition 6.4. Define the subsequence $t_{r_{n}}^{(1-j)}$ as follows:

- $t_{r_{1}}^{(1-j)}$ is the time of the latest occurring cull on patch $1-j$ to occur no later than the first cull on patch $j$, that is, $r_{1}$ is the greatest integer such that $t_{r_{1}}^{(1-j)} \leq t_{1}^{(j)}$ but $t_{r_{1}+1}^{(1-j)}>t_{1}^{(j)}$

- for $n \geq 1$, let $t_{r_{n+1}}^{(1-j)}$ be the time of the first cull on patch $1-j$ occurring strictly after $t_{r_{n}}^{(1-j)}+2 \tau$. 


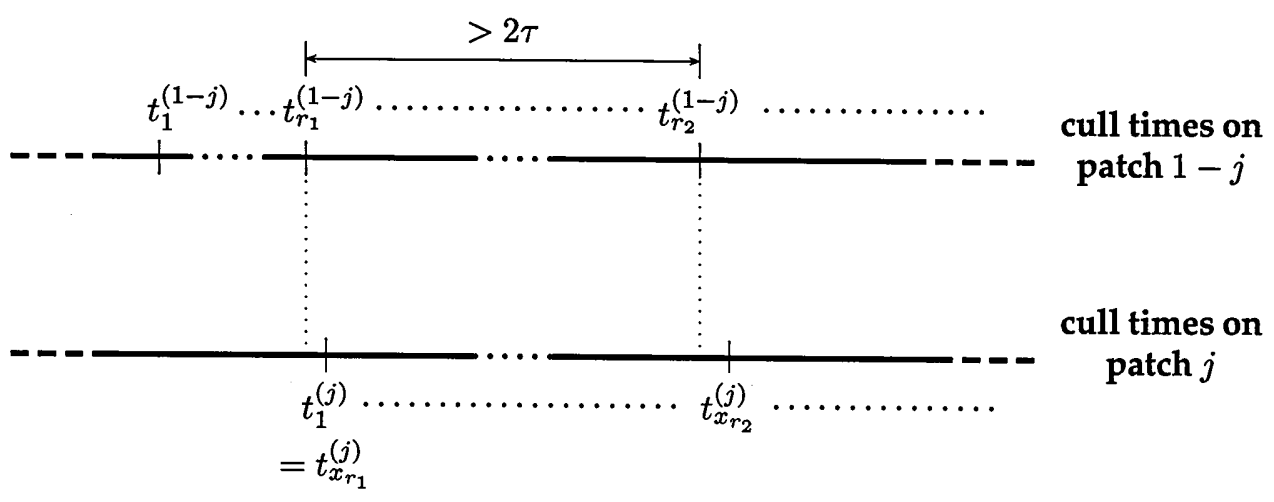

time

Figure 6.1: Early terms in the subsequences described in definitions 6.4 and 6.5.

Definition 6.5. Let $t_{x_{n}}^{(j)}$ for $n \geq 1$ be the time of the earliest occurring cull on patch $j$ such that $t_{x_{n}}^{(j)} \geq t_{n}^{(1-j)}$. In other words, $x_{n}$ is the smallest integer such that $t_{x_{n}}^{(j)} \geq t_{n}^{(1-j)}$.

No inconsistencies arise from combining the assumptions in definitions 6.4 and 6.5 with those in definitions 6.2 and 6.3 and condition (6.77). It should be understood that in the subsequence $t_{r_{n}}^{(1-j)}, n \geq 1$, the " $r$ " is fixed and is included only to remind us that the $t_{r_{n}}^{(1-j)}$ are a subsequence of the culling times $t_{r}^{(1-j)}, r \geq 1$. Similarly the " $x$ " is fixed in the subsequence $t_{x_{n}}^{(j)}, n \geq 1$.

To faciliate familiarity with the subsequences of definitions 6.4 and 6.5 , we illustrate some of the early terms in figure 6.1.

From definitions 6.4 and 6.5, we notice in particular that $t_{1}^{(j)}=t_{x_{1}}^{(j)}=t_{x_{2}}^{(j)}=\cdots=$ $t_{x_{r_{1}}}^{(j)}$. We also notice the following lemma which will be needed in theorem 6.4:

Lemma 6.5. For $n \geq 1$, we have

$$
t_{x_{r_{n}}}^{(j)} \leq t_{r_{n+1}}^{(1-j)}-\tau
$$

Proof. By definition 6.3, culls begin on patch $1-j$ before they begin on patch $j$ or at exactly the same time as they begin on patch $j$. The first cull on patch $j$, which occurs at time $t_{x_{r_{1}}}^{(j)}$, must occur before time $t_{r_{1}}^{(1-j)}+\tau$. This follows from the definition of $t_{r_{1}}^{(1-j)}$ and the fact that consecutive culls on patch $1-j$ occur no further apart than $\tau$ time units by definition 6.2 (specifically by (6.28)). Thus:

$$
t_{x_{r_{1}}}^{(j)}<t_{r_{1}}^{(1-j)}+\tau
$$

Now by definition $6.5, t_{x_{r_{n}}}^{(j)}$ is the time of the earliest occurring cull on patch $j$ such that $t_{x_{r_{n}}}^{(j)} \geq t_{r_{n}}^{(1-j)}$. Also, we have just mentioned that culls begin on patch $j$ before $t_{r_{1}}^{(1-j)}+\tau$, which means they must begin before $t_{r_{2}}^{(1-j)}$ since $t_{r_{2}}^{(1-j)}>t_{r_{1}}^{(1-j)}+2 \tau$ by definition 6.4. After culls begin on patch $j$ they occur at least every $\tau$ time units 
thereafter by (6.28). Hence, for $n \geq 2$, we must have $t_{x_{r_{n}}}^{(j)}<t_{r_{n}}^{(1-j)}+\tau$. Combining this last inequality with (6.79) reveals:

$$
t_{x_{r_{n}}}^{(j)}-t_{r_{n}}^{(1-j)}<\tau \text { for } n \geq 1
$$

Also, by definition 6.4, we have, for $n \geq 1$,

$$
t_{r_{n+1}}^{(1-j)}-t_{r_{n}}^{(1-j)}>2 \tau
$$

From (6.80) and (6.81), we have, for $n \geq 1$,

In other words,

$$
\begin{gathered}
t_{x_{r_{n}}}^{(j)}<t_{r_{n}}^{(1-j)}+\tau<t_{r_{n+1}}^{(1-j)}-2 \tau+\tau . \\
\quad t_{x_{r_{n}}}^{(j)}<t_{r_{n}}^{(1-j)}+\tau<t_{r_{n+1}}^{(1-j)}-2 \tau+\tau . \\
t_{x_{r_{n}}}^{(j)}<t_{r_{n+1}}^{(1-j)}-\tau \text { for } n \geq 1 .
\end{gathered}
$$

So certainly inequality (6.78) holds.

Now when we considered culling on a single patch in chapter 3 , we constructed (in lemma 3.13), from the initial data, an upper bound for the population immediately prior to the onset of the culling regime. We used this upper bound in establishing that a culling regime can eradicate the pest in theorem 3.14 and corollary 3.1. A similar approach may be adopted when a pest lives on two patches. We will state and prove a lemma (lemma 6.6) giving an upper bound for the two populations prior to the onset of regime $j$ (which is the regime that does not start first), and then use this upper bound in proving the main theorem of this chapter (theorem 6.4).

Lemma 6.6. Suppose that the birth functions on both patches can be bounded above by linear functions: $b_{0}\left(w_{0}\right) \leq \Lambda_{0} w_{0}$ and $b_{1}\left(w_{1}\right) \leq \Lambda_{1} w_{1}$. Then we may define $\theta_{3}$ to be a positive constant as in corollary 6.1. Assume that $\theta_{3} \geq 1$. Let $j$ be as stated in definition 6.3. Suppose the initial data is bounded above as follows:

$$
\left.\begin{array}{rl}
w_{1-j}(t) & \leq U_{1-j} \\
w_{j}(t) & \leq U_{j}
\end{array}\right\} \quad \text { for } t \in[-\tau, 0]
$$

where $U_{1-j}$ and $U_{j}$ are positive constants. Since we know that $j=0$ or $j=1$, then $U_{1-j}$ and $U_{j}$ represent $U_{0}$ and $U_{1}$ in some order. Let $U=\max \left\{U_{0}, U_{1}\right\}$. Let $\alpha=\theta_{3}+e^{\lambda_{1} \tau}\left(1-\theta_{3}\right)$ where $\lambda_{1}$ is a negative constant defined in (6.31). Make the assumptions in definitions 6.2 and 6.4. Then:

$$
\left.\begin{array}{rl}
w_{1-j}(t) & \leq \alpha^{\frac{1}{\tau} t_{1}^{(1-j)}+r_{1}+1} U \\
w_{j}(t) & \leq \alpha^{\frac{1}{\tau} t_{1}^{(1-j)}+r_{1}+1} U
\end{array}\right\} \quad \text { for } t \in\left[t_{r_{1}}^{(1-j)}-\tau, t_{1}^{(j)}\right],
$$

where $t_{1}^{(1-j)}$ is the time of the earliest occurring cull on patch $1-j, t_{1}^{(j)}$ is the time of the earliest occurring cull on patch $j$, and the meaning of $r_{1}$ is clear from definition 6.4 .

Proof. Observe that we must have $q \tau<t_{1}^{(1-j)} \leq(q+1) \tau$ for some integer $q \geq 0$. Then we may split the time interval $\left[0, t_{1}^{(j)}\right]$ into the following subintervals:

$$
\begin{aligned}
& {[0, \tau],[\tau, 2 \tau],[2 \tau, 3 \tau], \ldots,\left[q \tau, t_{1}^{(1-j)}\right],} \\
& \quad\left[t_{1}^{(1-j)}, t_{2}^{(1-j)}\right],\left[t_{2}^{(1-j)}, t_{3}^{(1-j)}\right], \ldots,\left[t_{r_{1}-1}^{(1-j)}, t_{r_{1}}^{(1-j)}\right],\left[t_{r_{1}}^{(1-j)}, t_{1}^{(j)}\right] .
\end{aligned}
$$


By construction, no culls occur strictly within any of these subintervals.

Now $\theta_{3} \geq 1$ by assumption. For the constant $\alpha$ in the statement of the lemma, it is clear that $\alpha \geq 1$ when $\theta_{3} \geq 1$. It is also clear, when $\theta_{3} \geq 1$ and $0<\epsilon \leq 1$ and $0<a<b \leq a+\tau$, that:

$$
\alpha \geq \max _{t \in[a, b]}\left\{\theta_{3}+e^{\lambda_{1}(t-a)}\left(\epsilon-\theta_{3}\right)\right\}
$$

We now argue by induction on $n \geq 1$ that

$$
\left.\begin{array}{rl}
w_{1-j}(t) & \leq \alpha^{n} U \\
w_{j}(t) & \leq \alpha^{n} U
\end{array}\right\}
$$

on the $n$-th subinterval in (6.86).

Basis step. The first subinterval in (6.86) is $\left[0, t_{1}^{(1-j)}\right]$ if $q=0$ or $[0, \tau]$ if $q \geq 1$. For ease of reference, denote by $\left[t_{0}, t_{1}\right]$ this first subinterval. Using (6.87) and the fact that no culls occur at a time that is strictly contained within the first subinterval (as opposed to occurring at an endpoint), we can apply corollary 6.1 (with $a=t_{0}=0$, $\left.b=t_{1}, K_{0}=K_{1}=U, \epsilon_{0}=\epsilon_{1}=1\right)$ to find that:

$$
\left.\begin{array}{rl}
w_{1-j}(t) & \leq \alpha U \\
w_{j}(t) & \leq \alpha U
\end{array}\right\} \quad \text { on the first subinterval in (6.86). }
$$

Inductive step. Assume (inductive hypothesis) that (6.88) holds on the first $n$ subintervals in (6.86) for some $n \geq 1$. Denote by $\left[t_{n-1}, t_{n}\right]$ the $n$-th subinterval and if a cull occurs on patch $1-j$ at time $t_{n}$ then denote its strength by $\bar{\epsilon}_{n}$. Define $\epsilon_{0}$ and $\epsilon_{1}$ as follows:

$$
\begin{aligned}
& \epsilon_{0}=1 \text { and } \epsilon_{1}=\bar{\epsilon}_{n} \text { if } j=0 \\
& \epsilon_{1}=1 \text { and } \epsilon_{0}=\bar{\epsilon}_{n} \text { if } j=1 .
\end{aligned}
$$

By the inductive hypothesis and the fact that $\alpha \geq 1$ when $\theta_{3} \geq 1$, we know that:

$$
\left.\begin{array}{rl}
w_{1-j}(t) & \leq \alpha^{n} U \\
w_{j}(t) & \leq \alpha^{n} U
\end{array}\right\} \quad \text { for } t \in\left[t_{n}-\tau, t_{n}\right]
$$

As in the basis step, we may now apply corollary 6.1 (with $a=t_{n}, b=t_{n+1}$, $K_{0}=K_{1}=\alpha_{n} U$, and with $\epsilon_{0}$ and $\epsilon_{1}$ defined according to whether $j=0$ or $j=1$ as outlined in (6.90) and (6.91)) to obtain:

$$
\left.\begin{array}{c}
w_{1-j}(t) \leq \alpha\left(\alpha^{n} U\right)=\alpha^{n+1} U \\
w_{j-j}(t) \leq \alpha\left(\alpha^{n} U\right)=\dot{\alpha}^{n+1} U
\end{array}\right\} \quad \text { on the }(n+1) \text {-th subinterval. }
$$

Thus we $\left.w_{j}(t) \leq \alpha\left(\alpha^{n} U\right)=\alpha^{n+1} U\right\}$ in (6.86). Since $\alpha \geq 1$ when $\theta_{3} \geq 1$, a consequence of this induction is that

$$
\left.\begin{array}{rl}
w_{1-j}(t) & \leq \alpha^{p} U \\
w_{j}(t) & \leq \alpha^{p} U
\end{array}\right\} \quad \text { for } t \in\left[t_{r_{1}}^{(1-j)}-\tau, t_{1}^{(j)}\right],
$$


where $p$ is the number of subintervals in (6.86). But there are clearly $(q+1)+r_{1}$ such subintervals where $q$ satisfies $q \tau<t_{1}^{(1-j)} \leq(q+1) \tau$. Hence

$$
p=(q+1)+r_{1}<\frac{1}{\tau} t_{1}^{(1-j)}+r_{1}+1 .
$$

Combining (6.94) with (6.95) and using the fact that $\alpha \geq 1$ when $\theta_{3} \geq 1$ now yields (6.85), as required.

Finally we state and prove the main theorem of this chapter:

Theorem 6.4. Make the assumptions in lemma 6.6. Assume in addition that condition (6.77) holds and recall the subsequences given in definitions 6.4 and 6.5. Then:

$$
w_{1-j}(t) \leq \pi \text { for } t \in\left[t_{r_{1}}^{(1-j)}-\tau, t_{r_{1}}^{(1-j)}\right]
$$

where $\pi=\alpha^{\frac{1}{\tau} t_{1}^{(1-j)}+r_{1}+1} U$ where $\alpha$ is defined in lemma 6.6. Moreover we have:

w]

$$
\begin{gathered}
w_{1-j}(t) \leq \delta^{n} \pi \text { for } t \in\left[t_{r_{n}}^{(1-j)}, t_{r_{n+1}}^{(1-j)}\right] \\
w_{1-j}(t) \leq \delta^{n} \pi \text { for } t \in\left[t_{r_{n}}^{(1-j)}, t_{r_{n+1}}^{(1-j)}\right]
\end{gathered}
$$

and

$$
w_{j}(t) \leq \delta^{n} \pi \text { for } t \in\left[t_{x_{r_{n}}}^{(j)}, t_{x_{r_{n+1}}}^{(j)}\right]
$$

for $n \geq 1$ where $\delta$ is defined in (6.77).

Proof. First note that inequalities (6.96) and (6.97) follow immediately from lemma 6.6.

To establish (6.98) and (6.99), we will ultimately use induction on $n \geq 1$. A number of arguments must be made before the induction can be given.

We begin, then, by noting the following two possibilites:

(A1) $t_{r_{1}}^{(1-j)}<t_{1}^{(j)}$

(A2) $t_{r_{1}}^{(1-j)}=t_{1}^{(j)}$

Case (A1): $t_{r_{1}}^{(1-j)}<t_{1}^{(j)}$

Consider the time interval $\left[t_{r_{1}}^{(1-j)}, t_{1}^{(j)}\right]$. By the initial data (inequalities (6.96) and (6.97)) we have

$$
\left.\begin{array}{r}
w_{1-j}(t) \leq \pi \\
w_{j}(t) \leq \pi
\end{array}\right\} \quad \text { for } t \in\left[t_{r_{1}}^{(1-j)}-\tau, t_{r_{1}}^{(1-j)}\right]
$$

We therefore also have the "initial conditions" as follows:

$$
\left.\begin{array}{rl}
w_{1-j}\left(t_{r_{1}}^{(1-j)}\right) & \leq \epsilon_{r_{1}}^{(1-j)} \pi \\
w_{j}\left(t_{r_{1}}^{(1-j)}\right) & \leq \pi
\end{array}\right\}
$$


Finally, no culls occur in the time interval $\left(t_{r_{1}}^{(1-j)}, t_{1}^{(j)}\right)$ by definition 6.4 , and $t_{1}^{(j)}-$ $t_{r_{1}}^{(1-j)}<\tau$ by definition 6.4 and (6.79).

If $j=0$ then set $\epsilon_{0}=1$ and $\epsilon_{1}=\epsilon_{r_{1}}^{(1-j)}$. But if $j=1$ then set $\epsilon_{1}=1$ and $\epsilon_{0}=\epsilon_{r_{1}}^{(1-j)}$. Also set $a=t_{r_{1}}^{(1-j)}, b=t_{1}^{(j)}$, and $K_{0}=K_{1}=\pi$. If we use these parameter choices in corollary 6.1 then we obtain, for $t \in\left[t_{r_{1}}^{(1-j)}, t_{1}^{(j)}\right]$,

$$
w_{1-j}(t) \leq \pi\left(\epsilon_{r_{1}}^{(1-j)} e^{\lambda_{1}\left(t-t_{r_{1}}^{(1-j)}\right)}+\theta_{3}\left(1-e^{\lambda_{1}\left(t-t_{r_{1}}^{(1-j)}\right)}\right)\right),
$$

by which we deduce that:

$$
w_{1-j}\left(t_{1}^{(j)}\right) \leq \pi\left(\epsilon_{r_{1}}^{(1-j)} e^{\lambda_{1}\left(t_{1}^{(j)}-t_{r_{1}}^{(1-j)}\right)}+\theta_{3}\left(1-e^{\lambda_{1}\left(t_{1}^{(j)}-t_{r_{1}}^{(1-j)}\right)}\right)\right) .
$$

Since $\theta_{3} \geq 1$ and using (6.77), we may also deduce from (6.102) that:

$$
w_{1-j}(t) \leq \delta \pi \text { for } t \in\left[t_{r_{1}}^{(1-j)}, t_{1}^{(j)}\right]
$$

Also, by lemma 6.6 and the fact that $t_{r_{1}}^{(1-j)}<t_{1}^{(j)}$ by the assumption of case (A1), we have:

$$
w_{1-j}(t) \leq \pi \text { for } t \in\left[t_{1}^{(j)}-\tau, t_{1}^{(j)}\right] .
$$

Furthermore, using (6.97) and the fact that $t_{r_{1}}^{(1-j)}<t_{1}^{(j)}$ by the assumption of case (A1), we have:

$$
w_{j}(t) \leq \pi \text { for } t \in\left[t_{1}^{(j)}-\tau, t_{1}^{(j)}\right]
$$

from which we find:

$$
w_{j}\left(t_{1}^{(j)}\right) \leq \epsilon_{1}^{(j)} \pi
$$

Case (A2): $t_{r_{1}}^{(1-j)}=t_{1}^{(j)}$

Here inequalities (6.96) and (6.97) give us

$$
\begin{aligned}
w_{1-j}(t) & \leq \pi \text { for } t \in\left[t_{1}^{(j)}-\tau, t_{1}^{(j)}\right] \\
w_{j}(t) & \leq \pi \text { for } t \in\left[t_{1}^{(j)}-\tau, t_{1}^{(j)}\right] .
\end{aligned}
$$

From these last two inequalities we have:

$$
\begin{aligned}
w_{1-j}\left(t_{1}^{(j)}\right) & \leq \epsilon_{r_{1}}^{(1-j)} \pi \\
w_{j}\left(t_{1}^{(j)}\right) & \leq \epsilon_{1}^{(j)} \pi .
\end{aligned}
$$

The inequalities derived in cases (A1) and (A2) will be useful in the next part of the proof. At any particular time $t$, define $\alpha_{t}^{(1-j)}$ to be the time of the latest occurring cull on patch $1-j$ such that $\alpha_{t}^{(1-j)} \leq t$ and suppose it has strength $\epsilon_{\left(\alpha_{t}\right)}^{(1-j)}$.

Also at time $t$ define $\beta_{t}^{(j)}$ to be the time of the latest occurring cull on patch $j$ such that $\beta_{t}^{(j)} \leq t$ and suppose it has strength $\epsilon_{\left(\beta_{t}\right)}^{(j)}$. 
Then we claim that the following equations hold for any time $t \geq t_{1}^{(j)}$ :

$$
\begin{aligned}
w_{1-j}(t) & \leq \pi\left[\epsilon_{\left(\alpha_{t}\right)}^{(1-j)} e^{\lambda_{1}\left(t-\alpha_{t}^{(1-j)}\right)}+\theta_{3}\left(1-e^{\lambda_{1}\left(t-\alpha_{t}^{(1-j)}\right)}\right)\right] \\
w_{j}(t) & \leq \pi\left[\epsilon_{\left(\beta_{t}\right)}^{(j)} e^{\lambda_{1}\left(t-\beta_{t}^{(j)}\right)}+\theta_{3}\left(1-e^{\lambda_{1}\left(t-\beta_{t}^{(j)}\right)}\right)\right] .
\end{aligned}
$$

To establish (6.112) and (6.113), it helps to define intervals $\left[t_{i}^{*}, t_{i+1}^{*}\right)$ for $i \geq 1$ as follows:

- $t_{1}^{*}=t_{1}^{(j)}$

- $t_{i+1}^{*}$ is the time of the first cull on either patch to occur strictly after $t_{i}^{*}$.

Since culls have already started on both patches for $t \geq t_{1}^{(j)}$, and since (by definition 6.2) consecutive culls on patch $k(k=0,1)$ occur no further apart than $\tau$ time units, it is clear that $t_{i+1}^{*}-t_{i}^{*} \leq \tau$. Also, since $t_{i}^{(1-j)} \rightarrow \infty$ as $i \rightarrow \infty$ and $t_{i}^{(j)} \rightarrow \infty$ as $i \rightarrow \infty$, it is clear that $t_{i}^{*} \rightarrow \infty$ as $i \rightarrow \infty$.

We now prove that (6.112) and (6.113) hold for $t \geq t_{1}^{(j)}$ by showing inductively that they hold on the intervals $\left[t_{i}^{*}, t_{i+1}^{*}\right)$ for $i \geq 1$.

Basis step. Our argument depends on which of case (A1) or (A2) holds.

Case (A1). The reader should be fairly well acquainted with how to apply corollary 6.1 by now, so in applying it henceforth we shall not be excessively detailed. Using (6.105), (6.103), (6.106), (6.107), and corollary 6.1, we obtain, for $t \in\left[t_{1}^{(j)}, t_{2}^{*}\right)$ :

$$
\begin{array}{r}
w_{1-j}(t) \leq \pi\left[\left(\epsilon_{r_{1}}^{(1-j)} e^{\lambda_{1}\left(t_{1}^{(j)}-t_{r_{1}}^{(1-j)}\right)}+\theta_{3}\left(\not 1-e^{\lambda_{1}\left(t_{1}^{(j)}-t_{r_{1}}^{(1-j)}\right)}\right)\right) e^{\lambda_{1}\left(t-t_{1}^{(j)}\right)}\right. \\
\left.+\theta_{3}\left(1-e^{\lambda_{1}\left(t-t_{1}^{(j)}\right)}\right)\right] .
\end{array}
$$

This implies, for $t \in\left[t_{1}^{(j)}, t_{2}^{*}\right)$ :

$$
w_{1-j}(t) \leq \pi\left(\epsilon_{r_{1}}^{(1-j)} e^{\lambda_{1}\left(t-t_{r_{1}}^{(1-j)}\right)}+\theta_{3}\left(1-e^{\lambda_{1}\left(t-t_{r_{1}}^{(1-j)}\right)}\right)\right) .
$$

This is the required result for $w_{1-j}(t)$, since at any time $t \in\left[t_{1}^{(j)}, t_{2}^{*}\right)$, the most recent cull on patch $1-j$ occurring at a time $\leq t$ is the one occurring at $t_{r_{1}}^{(1-j)}$ by the assumption of case (A1) and the definition of $t_{r_{1}}^{(1-j)}$.

Corollary 6.1 also gives, for $t \in\left[t_{1}^{(j)}, t_{2}^{*}\right)$,

$$
w_{j}(t) \leq \pi\left(\epsilon_{1}^{(j)} e^{\lambda_{1}\left(t-t_{1}^{(j)}\right)}+\theta_{3}\left(1-e^{\lambda_{1}\left(t-t_{1}^{(j)}\right)}\right)\right),
$$

which is the result required for $w_{j}(t)$.

The basis step is complete for case (A1). 
Case (A2). Using (6.108), (6.109), (6.110), (6.111), and corollary 6.1, we obtain, for $t \in\left[t_{1}^{(j)}, t_{2}^{*}\right]:$

$$
\begin{aligned}
w_{1-j}(t) & \leq \pi\left(\epsilon_{r_{1}}^{(1-j)} e^{\lambda_{1}\left(t-t_{r_{1}}^{(1-j)}\right)}+\theta_{3}\left(1-e^{\lambda_{1}\left(t-t_{r_{1}}^{(1-j)}\right)}\right)\right) \\
w_{j}(t) & \leq \pi\left(\epsilon_{1}^{(j)} e^{\lambda_{1}\left(t-t_{1}^{(j)}\right)}+\theta_{3}\left(1-e^{\lambda_{1}\left(t-t_{1}^{(j)}\right)}\right)\right) .
\end{aligned}
$$

But these are the results required on the basis step for case (A2).

The basis step is complete.

Inductive step. Both cases (A1) and (A2) are covered by the same argument. The first $m$ time intervals are $\left[t_{1}^{*}, t_{2}^{*}\right), \ldots,\left[t_{m}^{*}, t_{m+1}^{*}\right)$. Assume (inductive hypothesis) that (6.112) and (6.113) hold on the first $m \geq 1$ time intervals. But then, using the assumption that $\theta_{3} \geq 1$ and (6.77), we have:

$$
\left.\begin{array}{r}
w_{1-j}(t) \leq \delta \pi \leq \pi \\
w_{j}(t) \leq \delta \pi \leq \pi
\end{array}\right\} \quad \text { for } t \in\left[t_{1}^{*}, t_{2}^{*}\right), \ldots,\left[t_{m}^{*}, t_{m+1}^{*}\right) .
$$

By (6.96), (6.97), (6.119), and the obvious fact that $w_{1-j}\left(t_{m+1}^{*}\right) \leq w_{1-j}\left(t_{m+1}^{*-}\right)$ and $w_{j}\left(t_{m+1}^{*}\right) \leq w_{j}\left(t_{m+1}^{*-}\right)$ where $t_{m+1}^{*-}$ is the time "momentarily" before $t_{m+1}^{*}$, we have the following "initial data":

$$
\left.\begin{array}{r}
w_{1-j}(t) \leq \pi \\
w_{j}(t) \leq \pi
\end{array}\right\} \quad \text { for } t \in\left[t_{m+1}^{*}-\tau, t_{m+1}^{*}\right] .
$$

We need to show that (6.112) and (6.113) hold on $\left[t_{m+1}^{*}, t_{m+2}^{*}\right)$. There are three possibilities:

(B1) At time $t_{m+1}^{*}$, a cull occurs on patch $1-j$ but not on patch $j$

(B2) At time $t_{m+1}^{*}$, a cull occurs on patch $j$ but not on patch $1-j$

(B3) At time $t_{m+1}^{*}$, a cull occurs on patch $1-j$ and a cull occurs on patch $j$.

Case (B1) At time $t_{m+1}^{*}$, a cull occurs on patch $1-j$ but not on patch $j$.

The latest occurring cull on patch $1-j$ which occurs at a time $\leq t_{m+1}^{*}$ occurs at time $t_{m+1}^{*}$. Hence for any $t$ in the interval $\left[t_{m+1}^{*}, t_{m+2}^{*}\right)$ we have $\alpha_{t}^{(1-j)}=t_{m+1}^{*}$. This cull has strength $\epsilon_{\left(\alpha_{t}\right)}^{(1-j)}$ by definition. Using (6.120) we can therefore write:

$$
w_{1-j}\left(t_{m+1}^{*}\right) \leq \epsilon_{\left(\alpha_{t}\right)}^{(1-j)} \pi
$$

We also know by (6.120) that $w_{j}\left(t_{m+1}^{*}\right) \leq \pi$. Using this, and also using (6.121) and (6.120), we find by corollary 6.1 that, for $t \in\left[t_{m+1}^{*}, t_{m+2}^{*}\right)$,

$$
w_{1-j}(t) \leq \pi\left(\epsilon_{\left(\alpha_{t}\right)}^{(1-j)} e^{\lambda_{1}\left(t-t_{m+1}^{*}\right)}+\theta_{3}\left(1-e^{\lambda_{1}\left(t-t_{m+1}^{*}\right)}\right)\right) .
$$


But we have seen that $\alpha_{t}^{(1-j)}=t_{m+1}^{*}$ in the present case (case (B1)), so (6.122) is the same as (6.112). Thus (6.112) holds on $\left[t_{m+1}^{*}, t_{m+2}^{*}\right)$, as required.

By construction, no culls occur at any time $t \in\left(t_{m}^{*}, t_{m+1}^{*}\right)$. But then the latest occurring cull on patch $j$ to occur at a time $\leq t$ for any $t \in\left(t_{m}^{*}, t_{m+1}^{*}\right)$ will occur at a time $\leq t_{m}^{*}$. Denote the time of this cull by $\beta_{t}^{(j)}$ and its strength by $\epsilon_{\left(\beta_{t}\right)}^{(j)}$. Then, by the inductive hypothesis, we can say that, for $t \in\left(t_{m}^{*}, t_{m+1}^{*}\right)$ :

$$
w_{j}(t) \leq \pi\left[\epsilon_{\left(\beta_{t}\right)}^{(j)} e^{\lambda_{1}\left(t-\beta_{t}^{(j)}\right)}+\theta_{3}\left(1-e^{\lambda_{1}\left(t-\beta_{t}^{(j)}\right)}\right)\right] .
$$

Hence:

$$
\begin{aligned}
w_{j}\left(t_{m+1}^{*}\right) & \leq w_{j}\left(t_{m+1}^{*-}\right) \\
& \leq \pi\left[\epsilon_{\left(\beta_{t}\right)}^{(j)} e^{\lambda_{1}\left(t_{m+1}^{*}-\beta_{t}^{(j)}\right)}+\theta_{3}\left(1-e^{\lambda_{1}\left(t_{m+1}^{*}-\beta_{t}^{(j)}\right)}\right)\right],
\end{aligned}
$$

where $t_{m+1}^{*-}$ is the time "momentarily" before time $t_{m+1}^{*}$. Notice that, because of the assumption of case (B1), the latest occurring cull on patch $j$ to occur at a time $\leq t$ for any $t \in\left[t_{m+1}^{*}, t_{m+2}^{*}\right)$ will occur at the same time as the latest occurring cull on patch $j$ to occur at a time $\leq t$ where $t \in\left(t_{m}^{*}, t_{m+1}^{*}\right)$. Thus $\beta_{t}^{(j)}$ and $\epsilon_{\left(\beta_{t}\right)}^{(j)}$ in (6.124) may be interpreted as the time and strength of the latest occurring cull on patch $j$ to occur at a time $\leq t$ where $t \in\left[t_{m+1}^{*}, t_{m+2}^{*}\right)$.

From (6.120), we know that $w_{1-j}\left(t_{m+1}^{*}\right) \leq \pi$. Using this, as well as (6.124) and (6.120), we find by corollary 6.1 that, for $t \in\left[t_{m+1}^{*}, t_{m+2}^{*}\right)$,

$$
\begin{array}{r}
w_{j}(t) \leq \pi\left[\left(\epsilon_{\left(\beta_{t}\right)}^{(j)} e^{\lambda_{1}\left(t_{m+1}^{*}-\beta_{t}^{(j)}\right)}+\theta_{3}\left(\not 1-e^{\lambda_{1}\left(t_{m+1}^{*}-\beta_{t}^{(j)}\right)}\right)\right) e^{\lambda_{1}\left(t-t_{m+1}^{*}\right)}\right. \\
\left.+\theta_{3}\left(1-e^{\lambda_{1}\left(t-t_{m+1}^{*}\right)}\right)\right] .
\end{array}
$$

This implies, for $t \in\left[t_{m+1}^{*}, t_{m+2}^{*}\right)$ :

$$
w_{j}(t) \leq \pi\left[\epsilon_{\left(\beta_{t}\right)}^{(j)} e^{\lambda_{1}\left(t-\beta_{t}^{(j)}\right)}+\theta_{3}\left(1-e^{\lambda_{1}\left(t-\beta_{t}^{(j)}\right)}\right)\right] .
$$

This is the same as (6.113).

Hence the inductive step is established for case (B1).

Case (B2) At time $t_{m+1}^{*}$, a cull occurs on patch $j$ but not on patch $1-j$.

The latest occurring cull on patch $j$ which occurs at a time $\leq t_{m+1}^{*}$ occurs at time $t_{m+1}^{*}$. Hence for any $t$ in the interval $\left[t_{m+1}^{*}, t_{m+2}^{*}\right)$ we have $\beta_{t}^{(j)}=t_{m+1}^{*}$. This cull has strength $\epsilon_{\left(\beta_{t}\right)}^{(j)}$ by definition. Using (6.120) we can therefore write:

$$
w_{j}\left(t_{m+1}^{*}\right) \leq \epsilon_{\left(\beta_{t}\right)}^{(j)} \pi
$$


We also know by (6.120) that $w_{1-j}\left(t_{m+1}^{*}\right) \leq \pi$. Using this, and also using (6.127) and (6.120), we find by corollary 6.1 that, for $t \in\left[t_{m+1}^{*}, t_{m+2}^{*}\right)$,

$$
w_{j}(t) \leq \pi\left(\epsilon_{\left(\beta_{t}\right)}^{(j)} e^{\lambda_{1}\left(t-t_{m+1}^{*}\right)}+\theta_{3}\left(1-e^{\lambda_{1}\left(t-t_{m+1}^{*}\right)}\right)\right) .
$$

But we have seen that $\beta_{t}^{(j)}=t_{m+1}^{*}$ in the present case (case (B2)), so (6.128) is the same as (6.113). Thus (6.113) holds on $\left[t_{m+1}^{*}, t_{m+2}^{*}\right)$, as required.

By design, no culls occur at any time $t \in\left(t_{m}^{*}, t_{m+1}^{*}\right)$. But then the latest occurring cull on patch $1-j$ to occur at a time $\leq t$ for any $t \in\left(t_{m}^{*}, t_{m+1}^{*}\right)$ will occur at a time $\leq t_{m}^{*}$. Denote the time of this cull by $\alpha_{t}^{(j)}$ and its strength by $\epsilon_{\left(\alpha_{t}\right)}^{(1-j)}$. Then, by the inductive hypothesis, we can say that, for $t \in\left(t_{m}^{*}, t_{m+1}^{*}\right)$ :

$$
w_{1-j}(t) \leq \pi\left[\epsilon_{\left(\alpha_{t}\right)}^{(1-j)} e^{\lambda_{1}\left(t-\alpha_{t}^{(1-j)}\right)}+\theta_{3}\left(1-e^{\lambda_{1}\left(t-\alpha_{t}^{(1-j)}\right)}\right)\right]
$$

Hence:

$$
\begin{aligned}
w_{1-j}\left(t_{m+1}^{*}\right) & \leq w_{1-j}\left(t_{m+1}^{*-}\right) \\
& \leq \pi\left[\epsilon_{\left(\alpha_{t}\right)}^{(1-j)} e^{\lambda_{1}\left(t_{m+1}^{*}-\alpha_{t}^{(1-j)}\right)}+\theta_{3}\left(1-e^{\lambda_{1}\left(t_{m+1}^{*}-\alpha_{t}^{(1-j)}\right)}\right)\right],
\end{aligned}
$$

where $t_{m+1}^{*-}$ is the time "momentarily" before time $t_{m+1}^{*}$. Notice that, because of the assumption of case (B2), the latest occurring cull on patch $1-j$ to occur at a time $\leq t$ for any $t \in\left[t_{m+1}^{*}, t_{m+2}^{*}\right)$ will occur at the same time as the latest occurring cull on patch $1-j$ to occur at a time $\leq t$ where $t \in\left(t_{m}^{*}, t_{m+1}^{*}\right)$. Thus $\alpha_{t}^{(j)}$ and $\epsilon_{\left(\alpha_{t}\right)}^{(1-j)}$ in (6.130) may be interpreted as the time and strength of the latest occurring cull on patch $1-j$ to occur at a time $\leq t$ where $t \in\left[t_{m+1}^{*}, t_{m+2}^{*}\right)$.

From (6.120), we know that $w_{j}\left(t_{m+1}^{*}\right) \leq \pi$. Using this, as well as (6.130) and (6.120), we find by corollary 6.1 that, for $t \in\left[t_{m+1}^{*}, t_{m+2}^{*}\right)$,

$$
\begin{array}{r}
w_{1-j}(t) \leq \pi\left[\left(\epsilon_{\left(\alpha_{t}\right)}^{(1-j)} e^{\lambda_{1}\left(t_{m+1}^{*}-\alpha_{t}^{(1-j)}\right)}+\theta_{3}\left(\not 1-e^{\lambda_{1}\left(t_{m+1}^{*}-\alpha_{t}^{(1-j)}\right)}\right)\right) e^{\lambda_{1}\left(t-t_{m+1}^{*}\right)}\right. \\
\left.+\theta_{3}\left(1-e^{\lambda_{1}\left(t-t_{m+1}^{*}\right)}\right)\right]
\end{array}
$$

This implies, for $t \in\left[t_{m+1}^{*}, t_{m+2}^{*}\right)$ :

$$
w_{1-j}(t) \leq \pi\left[\epsilon_{\left(\alpha_{t}\right)}^{(1-j)} e^{\lambda_{1}\left(t-\alpha_{t}^{(1-j)}\right)}+\theta_{3}\left(1-e^{\lambda_{1}\left(t-\alpha_{t}^{(1-j)}\right)}\right)\right] .
$$

This is the same as (6.112).

Hence the inductive step is established for case (B2).

Case (B3) At time $t_{m+1}^{*}$, a cull occurs on patch $1-j$ and a cull occurs on patch $j$.

The latest occurring cull on patch $1-j$ which occurs at a time $\leq t_{m+1}^{*}$ occurs at time $t_{m+1}^{*}$. Hence for $t$ in the interval $\left[t_{m+1}^{*}, t_{m+2}^{*}\right)$ we have $\alpha_{t}^{(1-j)}=t_{m+1}^{*}$. This cull has strength $\epsilon_{\left(\alpha_{t}\right)}^{(1-j)}$ by definition. Using (6.120) we can therefore write:

$$
w_{1-j}\left(t_{m+1}^{*}\right) \leq \epsilon_{\left(\alpha_{t}\right)}^{(j)} \pi
$$


The latest occurring cull on patch $j$ which occurs at a time $\leq t_{m+1}^{*}$ occurs at time $t_{m+1}^{*}$. Hence for $t$ in the interval $\left[t_{m+1}^{*}, t_{m+2}^{*}\right)$ we have $\beta_{t}^{(j)}=t_{m+1}^{*}$. This cull has strength $\epsilon_{\left(\beta_{t}\right)}^{(j)}$ by definition. Using (6.120) we can therefore write:

$$
w_{j}\left(t_{m+1}^{*}\right) \leq \epsilon_{\left(\beta_{t}\right)}^{(j)} \pi .
$$

Using (6.133), (6.134), and (6.120), we find by corollary 6.1 that, for $t \in\left[t_{m+1}^{*}, t_{m+2}^{*}\right)$ :

$$
\begin{aligned}
w_{1-j}(t) & \leq K\left(\epsilon_{\left(\alpha_{t}\right)}^{(1-j)} e^{\lambda_{1}\left(t-t_{m+1}^{*}\right)}+\theta_{3}\left(1-e^{\lambda_{1}\left(t-t_{m+1}^{*}\right)}\right)\right) \\
w_{j}(t) & \leq K\left(\epsilon_{\left(\beta_{t}\right)}^{(j)} e^{\lambda_{1}\left(t-t_{m+1}^{*}\right)}+\theta_{3}\left(1-e^{\lambda_{1}\left(t-t_{m+1}^{*}\right)}\right)\right) .
\end{aligned}
$$

But we have seen that $\alpha_{t}^{(1-j)}=t_{m+1}^{*}$ in the present case (case (B3)), so (6.135) is the same as (6.112). Thus (6.112) holds on $\left[t_{m+1}^{*}, t_{m+2}^{*}\right)$, as required.

We have also seen that $\beta_{t}^{(j)}=t_{m+1}^{*}$ in the present case (case (B3)), so (6.136) is the same as (6.113). Thus (6.113) holds on $\left[t_{m+1}^{*}, t_{m+2}^{*}\right)$, as required.

Hence the inductive step is established for case (B3). Hence the induction itself is complete.

Thus we have established that (6.112) and (6.113) hold for $t \geq t_{1}^{(j)}$. But then it follows, by the assumption that $\theta_{3} \geq 1$ and by (6.77), that:

$$
\left.\begin{array}{rl}
w_{1-j}(t) & \leq \delta \pi \\
w_{j}(t) & \leq \delta \pi
\end{array}\right\} \quad \text { for } t \geq t_{1}^{(j)} .
$$

Now if case (A1) holds (see start of proof), then by (6.104) and (6.137), we may deduce that

$$
w_{1-j}(t) \leq \delta \pi \text { for } t \in\left[t_{r_{1}}^{(1-j)}, t_{r_{2}}^{(1-j)}\right] \text {. }
$$

Otherwise case (A2) holds, in which case $t_{r_{1}}^{(1-j)}=t_{1}^{(j)}$, and then (6.138) automatically follows from (6.137).

We may also deduce automatically from (6.137) that:

$$
w_{j}(t) \leq \delta \pi \text { for } t \in\left[t_{x_{r_{1}}}^{(j)}, t_{x_{r_{2}}}^{(j)}\right]
$$

But (6.138) is the same as (6.98) for $n=1$ and (6.139) is the same as (6.99) for $n=1$. In other words, we have established the basis step in an induction on $n \geq 1$ that (6.98) and (6.99) hold for $n \geq 1$.

To complete the proof, then, we need to establish an inductive step. Our inductive hypothesis is to assume for some $n \geq 1$ that both (6.98) and (6.99) hold, that is, to assume:

$$
w_{1-j}(t) \leq \delta^{n} \pi \text { for } t \in\left[t_{r_{n}}^{(1-j)}, t_{r_{n+1}}^{(1-j)}\right]
$$

and

$$
w_{j}(t) \leq \delta^{n} \pi \text { for } t \in\left[t_{x_{r_{n}}}^{(j)}, t_{x_{r_{n+1}}}^{(j)}\right]
$$


Then we need to show that:

$$
\begin{gathered}
w_{1-j}(t) \leq \delta^{n+1} \pi \text { for } t \in\left[t_{r_{n+1}}^{(1-j)}, t_{r_{n+2}}^{(1-j)}\right], \\
w_{j}(t) \leq \delta^{n+1} \pi \text { for } t \in\left[t_{x_{r_{n+1}}}^{(j)}, t_{x_{r_{n+2}}}^{(j)}\right]
\end{gathered}
$$

To show that (6.142) and (6.143) follow from (6.140) and (6.141), we may use a very similar argument to that used to establish that (6.138) and (6.139) follow from (6.96) and (6.97). In particular the number $\pi$ is simply rescaled by a factor $\delta^{n}$.

By definition of the $t_{r_{n}}^{(1-j)}$, we know that $t_{r_{n+1}}^{(1-j)}-t_{r_{n}}^{(1-j)}>2 \tau$, so that:

$$
t_{r_{n+1}}^{(1-j)}>t_{r_{n}}^{(1-j)}+\tau \text {. }
$$

We also know, by definition of the $t_{r_{n}}^{(1-j)}$ and $t_{x_{r_{n}}}^{(j)}$, and the fact that consecutive culls on patch $k(k=0,1)$ occur no further apart than $\tau$ time units, that:

$$
\begin{aligned}
& t_{x_{r_{n+1}}}^{(j)} \geq t_{r_{n+1}}^{(1-j)}>t_{r_{n}}^{(1-j)}+2 \tau \\
& t_{x_{r_{n+1}}}^{(j)} \geq t_{r_{n+1}}^{(1-j)}>t_{r_{n}}^{(1-j)}+2 \tau \\
&>t_{x_{r_{n}}}^{(j)}+\tau \text { by }(6.80) .
\end{aligned}
$$

Therefore by (6.144) anı (u.1xu), we kiluw mat our new initial data" (inequalities (6.140) and (6.141)) are defined on time intervals wide enough (at least $\tau$ time units) for us to be able to use corollary 6.1 without error.

To be able to use corollary 6.1 as we did on the basis step, we also need (6.141) to hold on $\left[t_{r_{n+1}}^{(1-j)}-\tau, t_{x_{r_{n+1}}}^{(j)}\right]$. This will be true if $t_{x_{r_{n}}}^{(j)} \leq t_{r_{n+1}}^{(1-j)}-\tau$. But we can be sure of this latter inequality by lemma 6.5 .

This completes the inductive step.

This completes the proof.

Corollary 6.2. Let the birth function $b_{0}$ be any of three types under consideration - linear, Nicholson, or Allee. Make the same assumption for the birth function $b_{1}$ where $b_{1}$ need not be identical to $b_{0}$. Then $b_{0}(N) \leq \Lambda_{0} N$ and $b_{1}(N) \leq \Lambda_{1} N$ for positive constants $\Lambda_{0}, \Lambda_{1}$. Let $\theta_{3}$ be a positive constant as defined in corollary 6.1. In the model of (6.1), (6.3), and (6.4) we know by theorem 6.3 that if $\theta_{3}<1$ then the pest will go extinct on both patches in the absence of culling.

Furthermore, if $\theta_{3} \geq 1$ and impulsive culling regimes are carried out simultaneously on both patches as described in definition 6.2, then the pest will be eradicated on both patches (that is, $w_{0}(t) \rightarrow 0$ and $w_{1}(t) \rightarrow 0$ as $t \rightarrow \infty$ ) if the following additional condition holds:

$$
\delta_{k}=\sup _{i \geq 1}\left\{\epsilon_{i}^{(k)} e^{\lambda_{1}\left(t_{i+1}^{(k)}-t_{i}^{(k)}\right)}+\theta_{3}\left(1-e^{\lambda_{1}\left(t_{i+1}^{(k)}-t_{i}^{(k)}\right)}\right)\right\}<1,
$$

for $k=0,1$ and where $\lambda_{1}$ is a negative constant defined in (6.31).

Proof. We know by lemma 5.2 that Nicholson and Allee birth functions can be bounded above by linear functions. The success of the culling regimes subject to definition 6.2 and (6.146) (which is equivalent to (6.77)) when $\theta_{3} \geq 1$ is then an immediate consequence of theorem 6.4. 
Remark 1. Theorem 6.4 does not simply establish that successful culling regimes exist. It tells us that these regimes eradicate the pest in such a way that the populations on both patches are bounded above by a strictly monotonic decreasing sequence (assuming factors such as pesticide resistance do not alter the truth of our results). We can be sure, then, that when we implement these regimes, the pest populations will not flare up and destroy a crop or cause an epidemic before coming under our control. Notice the similarity of this remark to remark 2 at the end of subsection 3.4.1.

Remark 2. Theorem 6.4 trivially allows us to place an upper bound on the time it will take to reduce the pest populations to be below any particular levels. This is an extremely practical discovery. Incessant pesticide programs may lead to pesticide resistance and other problems (see section 2.3), so that in real-world scenarios it may be decided to apply pesticides only until the pest populations on both patches have been suitably reduced. Unending control programs are also likely to be more expensive than ephemeral ones. On a related note, the method of proof in theorem 6.4 will allow us to conclude in section 6.7 that eradication can be achieved in only finitely many culls when both birth functions are of Allee type.

Remark 3. We have derived conditions ensuring that our regimes succeed, but there is no reason per se to suppose that they are the least restrictive such conditions. There may be weaker regimes that also succeed, as suggested by a simulation below (see figure 6.4 in section 6.8). It would be a useful research question to find successful regimes that are weaker than ours.

Remark 4. Although we have proven that the pest will go extinct in the absence of culling when $\theta_{3}<1$, we have not established that the pest must always persist if $\theta_{3} \geq 1$. Therefore culling may not always be necessary when $\theta_{3} \geq 1$. Extinction and endemicity results have been discussed in chapter $5 \mathrm{but}$, as we noted at the end of that chapter, our results were by no means exhaustive.

\subsection{Analysis of the regimes}

We have said (final paragraph, section 6.4) that condition (6.77), which is equivalent to condition (6.146) in corollary 6.2, will hold if the inter-cull times on both patches are small enough. We explore condition (6.146) more precisely in this section in the context of a simple special case. So, suppose that consecutive culls on patch $k$ ( $k=$ $0,1)$ occur a fixed time $T_{k}>0$ apart. Since consecutive culls on patch $k$ occur no further apart than $\tau$ time units by definition 6.2 , then $T_{k} \leq \tau$. Recall that $\epsilon_{(k)}^{*}=$ $\sup _{i \geq 1} \epsilon_{i}^{(k)}$ for $k=0,1$ (see definition 6.2). Then (6.146) holds if:

$$
\delta_{k} \leq \epsilon_{(k)}^{*} e^{\lambda_{1} T_{k}}+\theta_{3}\left(1-e^{\lambda_{1} T_{k}}\right)<1 .
$$

Now a farmer (or other authority) may not have much control over how strong pesticides are manufactured to be, but they will presumably have some control over how frequently they can apply a pesticide. If the pesticide strengths are known, then the values of $\epsilon_{(0)}^{*}$ and $\epsilon_{(1)}^{*}$ are known, allowing us to to seek upper bounds for $T_{0}$ and $T_{1}$ by rearranging (6.147). Bearing in mind that $\epsilon_{(k)}^{*}<1$ and $T_{k} \leq \tau$ by definition 6.2 , and that culling is not needed unless $\theta_{3} \geq 1$, we trivially find that (6.147) holds if, for $k=0,1$, we have:

$$
\left.\begin{array}{l}
T_{k} \leq \tau \text { when } \theta_{3}=1 \\
T_{k}<\min \left\{\tau,\left(\frac{-1}{\lambda_{1}} \ln \left(\frac{\theta_{3}-\epsilon_{(k)}^{*}}{\theta_{3}-1}\right)\right)\right\} \text { when } \theta_{3}>1 .
\end{array}\right\}
$$



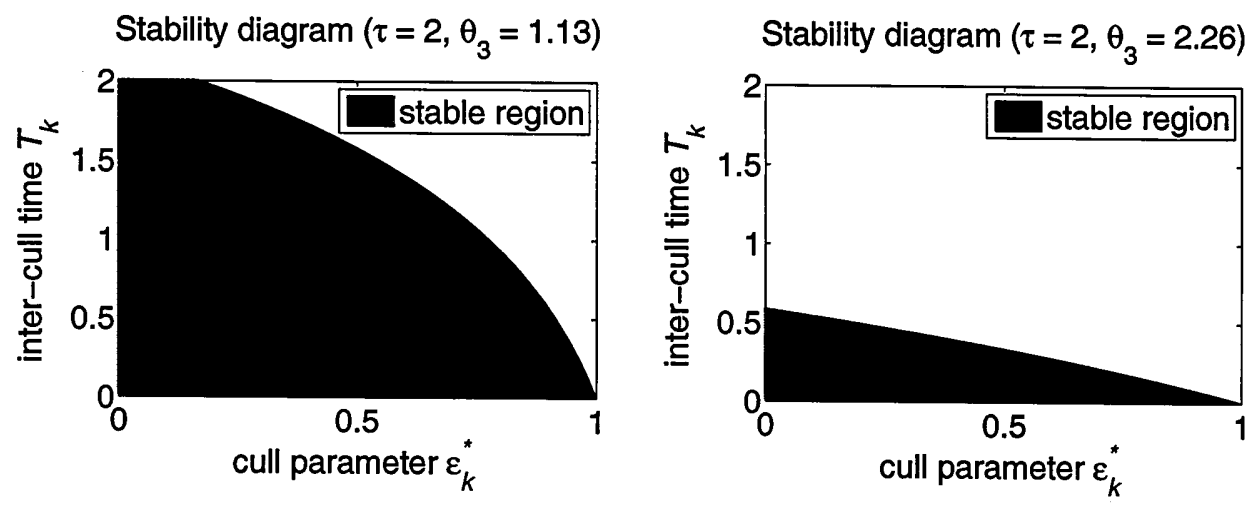

Figure 6.2: Stability diagrams for culling regimes. See section 6.6 for an explanation.

The formula (6.148) tells us how often the pesticide should be applied on patch $k$ $(k=0,1)$ in order to guarantee pest eradication. Notice, then, that as long as each cull has some effect (so that $\epsilon_{(k)}^{*}<1$ ), then our regimes ensure eradication on both patches as long as consecutive culls on patch $k(k=0,1)$ are performed sufficiently close in time (so that $T_{k}$ is small enough to satisfy (6.148)). An analogous result holds when the pest dwells on only one patch, as we saw in subsection 3.4.4.

To gain insight into how restrictive condition (6.147) is in the case $\theta_{3}>1$, we plot stability diagrams in figure 6.2. The shaded region in each plot indicates the part of the $T_{k}-\epsilon_{(k)}^{*}$ parameter space for which (6.147) holds. The shaded region is "stable" in the sense that a culling regime with parameters restricted to lie in the region will eradicate the pest. The boundaries of the stable region are $0<\epsilon_{(k)}^{*}<1,0<T_{k} \leq \tau$, and $\epsilon_{(k)}^{*} e^{\lambda_{1} T_{k}}+\theta_{3}\left(1-e^{\lambda_{1} T_{k}}\right)=1$. Note the exact similarity of the plots in figure 6.2 with those in figure 3.2. This similarity should not surprise us - our methods in this chapter have resembled those in chapter 3.

\subsection{Eradication in finitely many culls}

Recall corollary 5.1 in chapter 5 , which says that if both birth functions, $b_{0}$ and $b_{1}$, are of Allee-type, then there always exist minimum viable populations on the patches. In other words, there exist numbers $N_{k}>0(k=0,1)$ such that, if $w_{k}(t)<N_{k}$ for at least $\tau$ consecutive time units, then $w_{k}(t) \rightarrow 0$ as $t \rightarrow \infty$. Here $N_{0}$ and $N_{1}$ are any positive numbers satisfying $N_{0}+N_{1}<\psi$ where $\psi$ is a positive constant defined in terms of the model and birth function parameters in the statement of corollary 5.1.

Now we have seen (corollary 6.2) that culling is unnecessary when $\theta_{3}<1$ (where $\theta_{3}$ is a positive constant defined in terms of model and birth function parameters). Therefore, suppose $\theta_{3} \geq 1$. If culls occur on patch $k$ every $T_{k}$ time units, where $T_{k}>0$ satisfies (6.148), then theorem 6.4 ensures that it will take only a finite number of culls before $w_{k}(t)<N_{k}$ for all $t$ large enough (say, for all $t>T^{*}$ ) for any $N_{k}>0$. Certainly then, $w_{k}(t)<N_{k}$ for $t \in\left[T^{*}, T^{*}+\tau\right]$, so by the second sentence in this section we will automatically have $w_{k}(t) \rightarrow 0$ as $t \rightarrow \infty$, even if culling ceases at time $T^{*}+\tau$. In summary, only a finite number of culls is needed on both patches to ensure pest 
eradication when both birth functions are of Allee type. (An analogous result holds when the pest inhabits only one patch, as we saw in subsection 3.4.3.)

We can find explicit upper bounds for the numbers of culls needed on the patches to guarantee eradication. To this end, recall that patch $1-j(j=0,1)$ is the patch on which culling begins first (see definition 6.3). Recall also the subsequences $t_{r_{n}}^{(1-j)}$ and $t_{x_{r_{n}}}^{(j)}$ (definitions 6.4 and 6.5). Assume as in the last paragraph that $\theta_{3} \geq 1$ and that culls occur on patch $k$ every $T_{k}$ time units $(k=0,1)$ where $T_{k}>0$ satisfies (6.148). Make the assumptions in theorem 6.4 except that we intend to stop culling after $m_{1-j}$ culls on patch $1-j$ and after $m_{j}$ culls on patch $j$. We will find upper bounds (though not necessarily the least such bounds) on $m_{1-j}$ and $m_{j}$ such that, if there are only $m_{1-j}$ culls on patch $1-j$ and $m_{j}$ culls on patch $j$, then eradication will still occur. By assumption in theorem 6.4, the initial data is bounded above: $w_{1-j}(t) \leq U_{1-j}$ and $w_{j}(t) \leq U_{j}$ for $t \in[-\tau, 0]$. Theorem 6.4 also tells us (repeating inequalities (6.98) and (6.99)) that:

$$
w_{1-j}(t) \leq \delta^{n} \pi \text { for } t \in\left[t_{r_{n}}^{(1-j)}, t_{r_{n+1}}^{(1-j)}\right]
$$

and

$$
w_{j}(t) \leq \delta^{n} \pi \text { for } t \in\left[t_{x_{r_{n}}}^{(j)}, t_{x_{r_{n+1}}}^{(j)}\right]
$$

for $n \geq 1$, where $\pi>0$ is a constant defined in terms of initial data and culling and model parameters, and where $\delta$ is a constant (with $0<\delta<1$ ) defined in (6.77).

We have mentioned how, by corollary 5.1 , there are minimum viable populations on the patches. But in particular we note that if $U_{1-j}+U_{j}<\psi$ (where $\psi$ is defined in corollary 5.1) then the initial data will be low enough to ensure that the pest will go extinct without any culling. For the remainder of this section, then, we will assume that $U_{1-j}+U_{j} \geq \psi$. Since we have assumed that $\theta_{3} \geq 1$, we may trivially deduce that $\pi \geq U$ where $U=\max \left\{U_{1-j}, U_{j}\right\}$. Hence $2 \pi \geq \psi$.

Assume for the moment that culling occurs indefinitely on both patches. For $n$ large enough, say $n \geq \hat{n}$, we will have $\delta^{n} \pi<\frac{\psi}{2}$. But then by (6.149) and (6.150), we will have $w_{1-j}(t)+w_{j}(t)<\psi$ for $t \geq t_{x_{r_{\hat{n}}}}^{(j)}$ and in particular for $t \in\left[t_{x_{r_{n}}}^{(j)}, t_{x_{r_{\hat{n}}}}^{(j)}+\tau\right]$. We may stop culling then at time $t=t_{x_{r_{n}}}^{(j)}+\tau$ and the pest will necessarily go extinct on both patches by corollary 5.1 since the combined populations on both patches will have been bounded above by $\psi$ for at least $\tau$ consecutive time units.

Now how many culls are required on each patch if culls stop at time $t_{x_{r_{n}}}^{(j)}+\tau$ ? First we calculate an $\hat{n}$ such that $\delta^{\hat{n}} K<\frac{\psi}{2}$. We trivially find such an $\hat{n}$ to be:

$$
\hat{n}=\left\lfloor\frac{\ln \left(\frac{2 K}{\psi}\right)}{\ln \left(\frac{1}{\delta}\right)}\right\rfloor+1,
$$

where $\downarrow$ denotes the greatest integer function. In order for culling to continue at least until time $t_{x_{r_{\hat{n}}}}^{(j)}+\tau$, we ask for $m_{1-j}$ and $m_{j}$ to satisfy:

$$
\begin{aligned}
t_{1}^{(1-j)}+\left(m_{1-j}-1\right) T_{1-j} & >t_{x_{r_{\hat{n}}}}^{(j)}+\tau, \\
t_{1}^{(j)}+\left(m_{j}-1\right) T_{j} & >t_{x_{r_{\hat{n}}}}^{(j)}+\tau .
\end{aligned}
$$

Now by definition 6.4 we know that $t_{r_{n+1}}^{(1-j)}$ is the earliest occurring cull on patch $1-j$ to occur strictly after $t_{r_{n}}^{(1-j)}+2 \tau$. But since culls occur at least once every $\tau$ time units on patch $1-j$ (by definition 6.2), we must have $t_{r_{n+1}}^{(1-j)} \leq t_{r_{n}}^{(1-j)}+3 \tau$. Inductively, then, 
$t_{r_{\hat{n}}}^{(1-j)} \leq t_{r_{1}}^{(1-j)}+3(\hat{n}-1) \tau$. Again, since a cull occurs at least every $\tau$ time units, we must have $t_{r_{1}}^{(1-j)} \leq t_{1}^{(1-j)}+\left(r_{1}-1\right) \tau$. Hence $t_{r_{\hat{n}}}^{(1-j)} \leq t_{1}^{(1-j)}+\left(3(\hat{n}-1)+\left(r_{1}-1\right)\right) \tau$. By inequality (6.80) we also have $t_{x_{r_{\hat{n}}}}^{(j)}<t_{r_{\hat{n}}}^{(1-j)}+\tau$. The last two inequalities yield:

$$
t_{x_{r_{\hat{n}}}}^{(j)}+\tau \leq t_{1}^{(1-j)}+\left(3(\hat{n}-1)+r_{1}+1\right) \tau .
$$

Also by definition 6.4 , we have $t_{r_{1}}^{(1-j)} \leq t_{1}^{(j)}$, so we may write, by our various inequalities:

$$
\begin{aligned}
t_{x_{r_{\hat{n}}}}^{(j)}+\tau & <t_{r_{\hat{n}}}^{(1-j)}+2 \tau \\
& \leq t_{r_{1}}^{(1-j)}+(3(\hat{n}-1)+2) \tau \\
& \leq t_{1}^{(j)}+(3(\hat{n}-1)+2) \tau .
\end{aligned}
$$

Hence if

$$
m_{1-j}>1+\frac{\left(3(\hat{n}-1)+r_{1}+1\right) \tau}{T_{1-j}}
$$

then

$$
\begin{aligned}
t_{1}^{(1-j)}+\left(m_{1-j}-1\right) T_{1-j} & >t_{1}^{(1-j)}+\left(3(\hat{n}-1)+r_{1}+1\right) \tau \\
& >t_{x_{r_{\hat{n}}}}^{(j)}+\tau \text { by }(6.154),
\end{aligned}
$$

and if

$$
m_{j}>1+\frac{(3(\hat{n}-1)+2) \tau}{T_{j}}
$$

then

$$
\begin{aligned}
t_{1}^{(j)}+\left(m_{j}-1\right) T_{j} & >t_{1}^{(j)}+(3(\hat{n}-1)+2) \tau \\
& >t_{x_{r_{\hat{n}}}}^{(j)}+\tau \text { by }(6.155) .
\end{aligned}
$$

In other words, if we stop culling after $\left(m_{1-j}^{*}, m_{j}^{*}\right)$ culls on patches $(1-j, j)$ where

$$
\begin{aligned}
m_{1-j}^{*} & =2+\left\lfloor\frac{\left(3(\hat{n}-1)+r_{1}+1\right) \tau}{T_{1-j}}\right\rfloor, \\
m_{j}^{*} & =2+\left\lfloor\frac{(3(\hat{n}-1)+2) \tau}{T_{j}}\right\rfloor,
\end{aligned}
$$

then enough culls will have occurred to ensure eradication with no further culling. Since $\hat{n}, r_{1}, \tau, T_{1-j}$, and $T_{j}$ are known, (6.160) and (6.161) give us explicit finite upper bounds for the numbers of culls needed on patches $1-j$ and $j$ respectively to guarantee eradication.

\subsection{Simulations}

Since culling is unnecessary when $\theta_{3}<1$ (where $\theta_{3}$ is a positive constant defined in terms of model and birth function parameters in corollary 6.2), we conduct impulsive culling simulations only when $\theta_{3} \geq 1$. To conduct the simulations, we make some simple assumptions. First, in any particular simulation, let all culls on patch $k(k=$ 

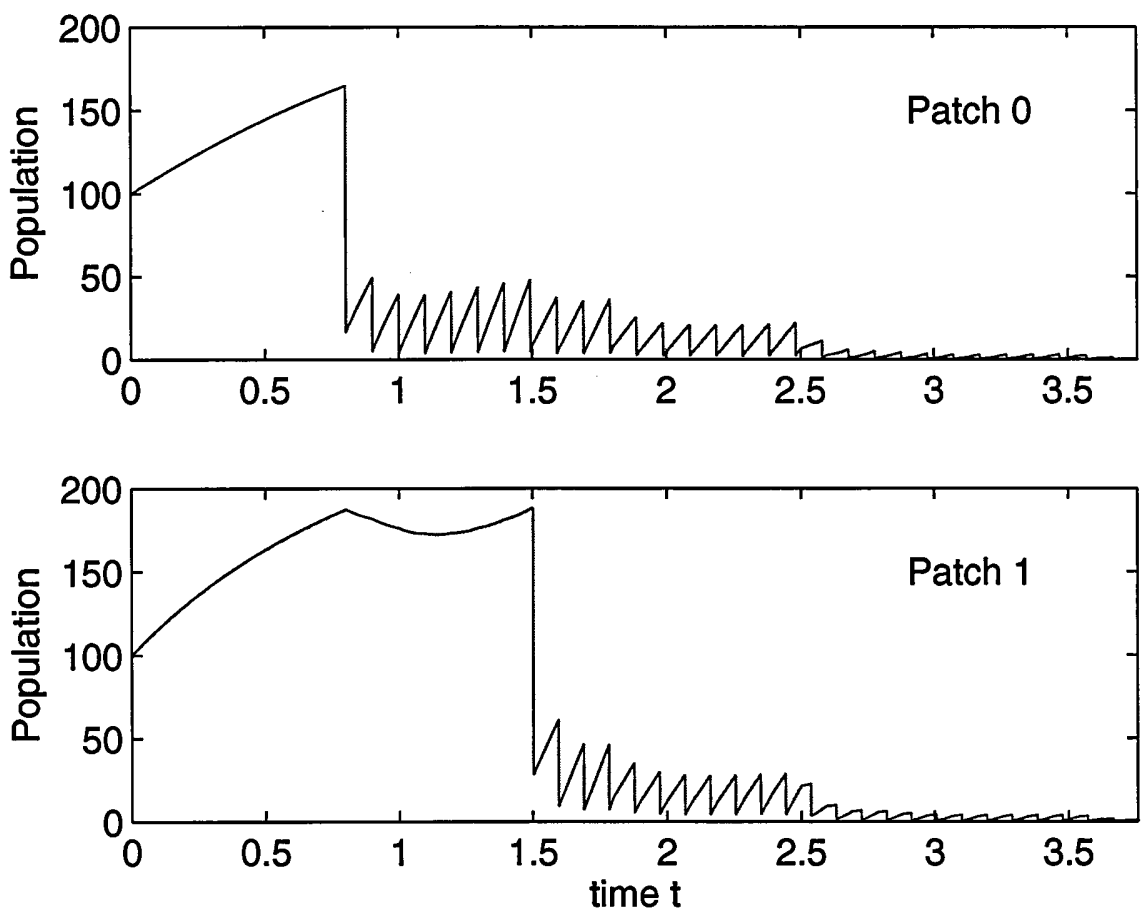

Figure 6.3: Culling when both birth functions are linear. Model and culling parameters are stated in paragraph 2 , section 6.8.

$0,1)$ have the same strength $\epsilon_{k}$ and let consecutive culls occur a fixed time $T_{k}>0$ apart, where $T_{k}$ satisfies equation (6.148). For different simulations, $\epsilon_{k}$ and $T_{k}$ are not necessarily the same.

In figure 6.3 we plot the populations on both patches when both birth functions are linear, specifically $b_{0}\left(w_{0}\right)=\Lambda_{0} w_{0}, b_{1}\left(w_{1}\right)=\Lambda_{1} w_{1}$ where $\Lambda_{0}=\Lambda_{1}=1$. The initial data is $w_{0}(t)=w_{1}(t)=100$ for $t \in[-\tau, 0]$ and the delay $\tau=1$. Parameter values are:

$$
A=2.1, B=1.1, C=1, D=1, E=1, F=1.9, G=1.2, H=1.4 \text {. }
$$

These yield $\theta_{3}=3.5559>1$ (where $\theta_{3}=\theta_{1}+\theta_{2}$ with $\theta_{1}>0$ defined in (6.45), and $\theta_{2}>0$ found by (6.46), (6.30), (6.31), and (6.32)). Culls on patch 0 start at time $t_{0}=0.8$, have strength $\epsilon_{0}=0.1$, and occur $T_{0}=0.0988$ time units apart. Culls on patch 1 start at time $t_{1}=1.5$, have strength $\epsilon_{1}=0.3$, and occur $T_{1}=0.0793$ time units apart. Note that $T_{0}$ and $T_{1}$ are chosen to satisfy the second expression in (6.148), thereby guaranteeing eradication by our results. Notice that in order to satisfy this expression, we had to choose $T_{0}$ and $T_{1}$ to be quite small.

Figure 6.4 also plots culling on both patches when both birth functions are linear. The initial data, the delay, and the model parameters are exactly the same as those used to create figure 6.3 but most of the culling parameters are different. The culling parameters in figure 6.4 are: $t_{0}=0.8, \epsilon_{0}=0.1, T_{0}=0.7, t_{1}=4.3, \epsilon_{1}=0.15, T_{1}=0.9$. Notice that the culling regimes defined by these parameter values are not guaranteed by our results to eradicate the pest on both patches. Nevetheless the simulation suggests that the populations may go extinct. This should not necessarily surprise us. 

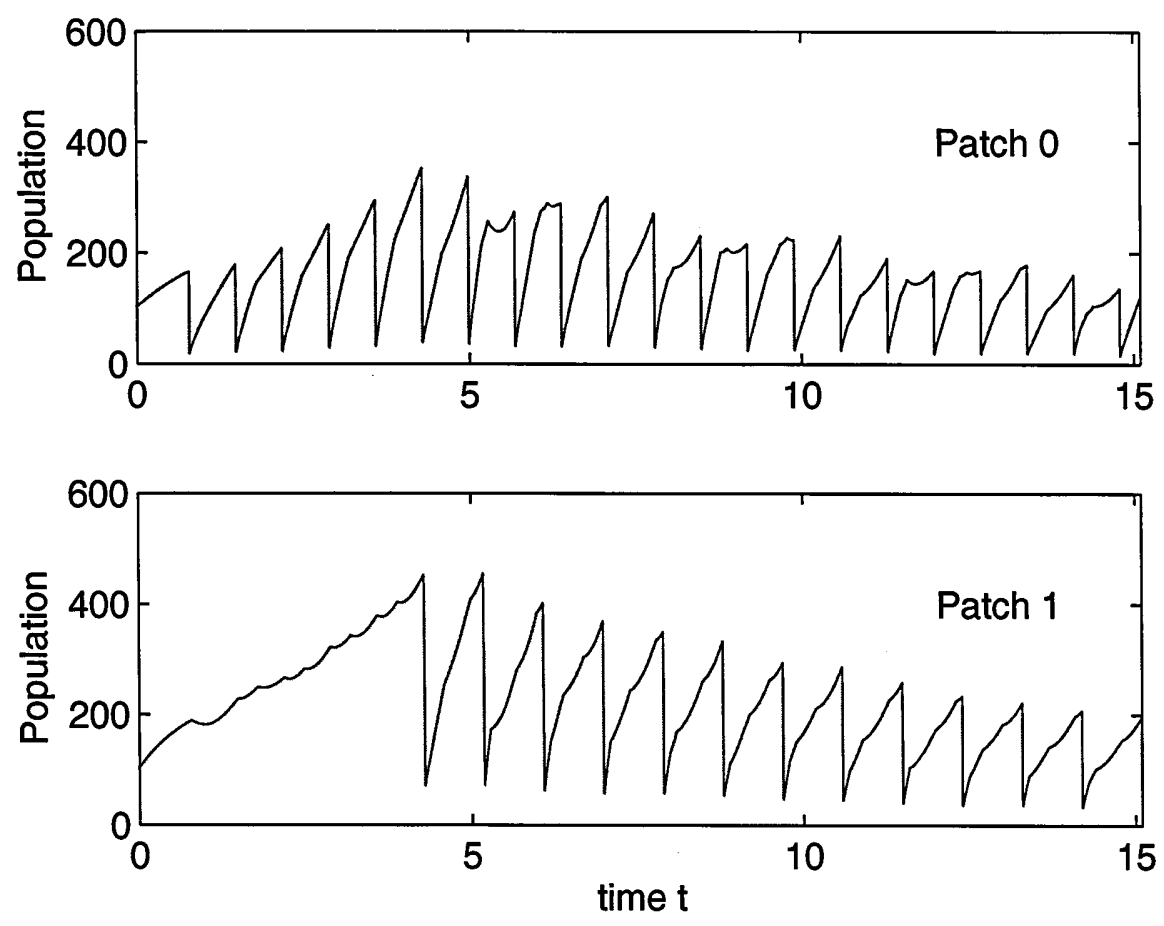

Figure 6.4: Culling when both birth functions are linear. Model and culling parameters are stated in section 6.8 .

We have proven that suitably strong culling regimes ensure eradication but we may not have discovered all possible successful impulsive culling regimes. Observe also that after culling has started on patch 0 but before culling has started on patch 1 , the populations on both patches show a general upwards trend, allowing us to visualise our conclusion in section 6.2 that culling on only one patch may sometimes not be enough to control the pest on either patch.

In figure 6.5 we plot the populations when both birth functions are of Nicholson type, specifically $b_{0}\left(w_{0}\right)=3 w_{0} e^{-0.05 w_{0}}$ and $b_{1}\left(w_{1}\right)=2 w_{1} e^{-0.1 w_{1}}$. The initial data is $\left(w_{0}(t), w_{1}(t)\right)=(20,25)$ for $t \in[-\tau, 0]$ and the delay $\tau=1$. The model parameters $A, B, C, \ldots, H$ are as stated in (6.162). Since the birth functions can be bounded above by linear functions (namely $b_{0}\left(w_{0}\right) \leq 3 w_{0}$ and $b_{1}\left(w_{1}\right) \leq 2 w_{1}$ ), we find (by (6.45) and (6.46) and the fact that $\theta_{3}=\theta_{1}+\theta_{2}$ ) that $\theta_{3}=7.3552$. Culls on patch 0 start at time $t_{0}=0.4$, have strength $\epsilon_{0}=0.3$, and occur $T_{0}=0.0342$ time units apart. Culls on patch 1 start at time $t_{1}=0.5$, have strength $\epsilon_{1}=0.35$, and occur $T_{1}=0.0319$ time units apart. Here $T_{0}$ and $T_{1}$ are chosen to satisfy (6.148), so that eradication is guaranteed by our results.

In figure 6.6 we plot the populations when both birth functions are of Allee type. To be specific, $b_{0}\left(w_{0}\right)=3 w_{0}^{2} e^{-0.2 w_{0}}$ and $b_{1}\left(w_{1}\right)=2 w_{1}^{2} e^{-0.25 w_{1}}$. The initial data is $\left(w_{0}(t), w_{1}(t)\right)=(20,25)$ for $t \in[-\tau, 0]$ and the delay $\tau=0.05$. The model parameters $A, B, C, \ldots, H$ are as stated in (6.162). Given that the birth functions can be bounded above by linear functions (namely $b_{0}\left(w_{0}\right) \leq \frac{3}{0.2 e} w_{0}$ and $b_{1}\left(w_{1}\right) \leq \frac{2}{0.25 e} w_{1}$ ), we find (by (6.45) and (6.46) and the fact that $\theta_{3}=\theta_{1}+\theta_{2}$ ) that $\theta_{3}=11.7080$. The 

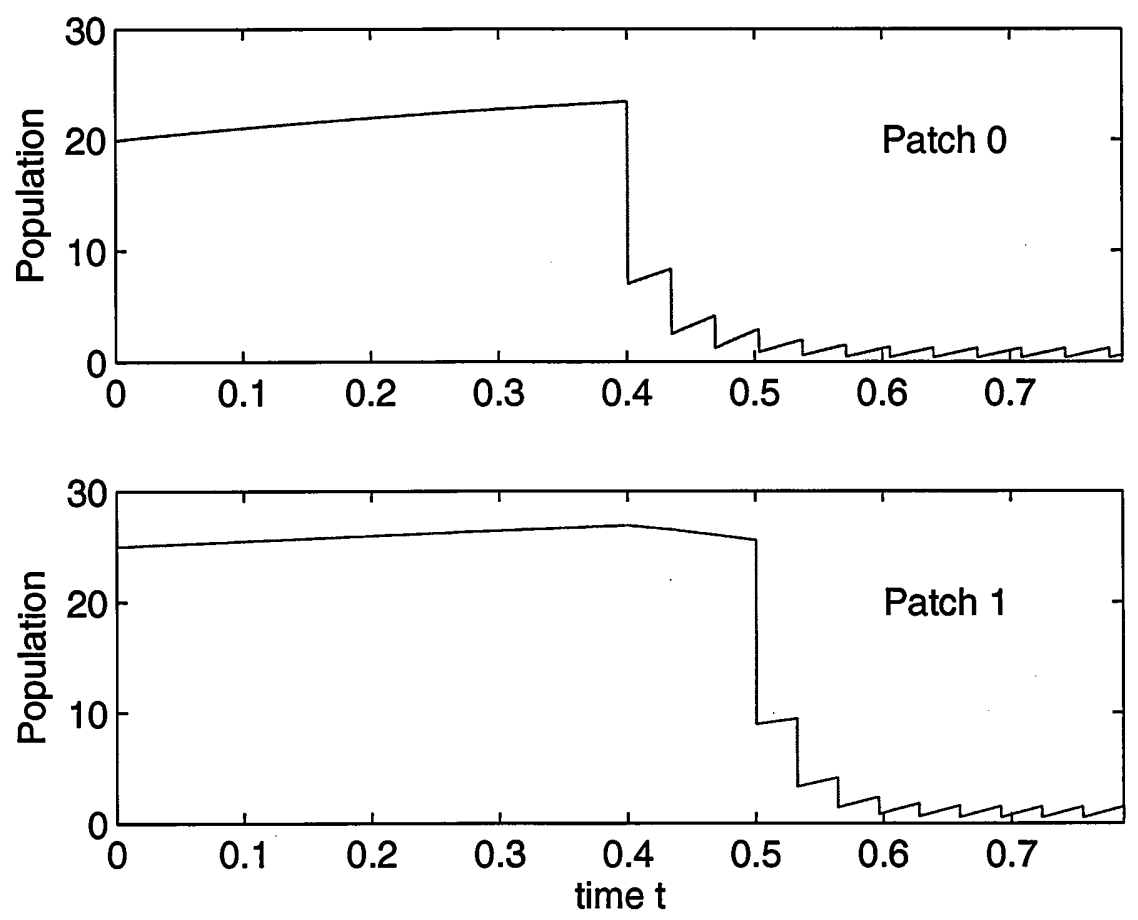

Figure 6.5: Culling when both birth functions are Nicholson. Model and culling parameters are stated in section 6.8 . 

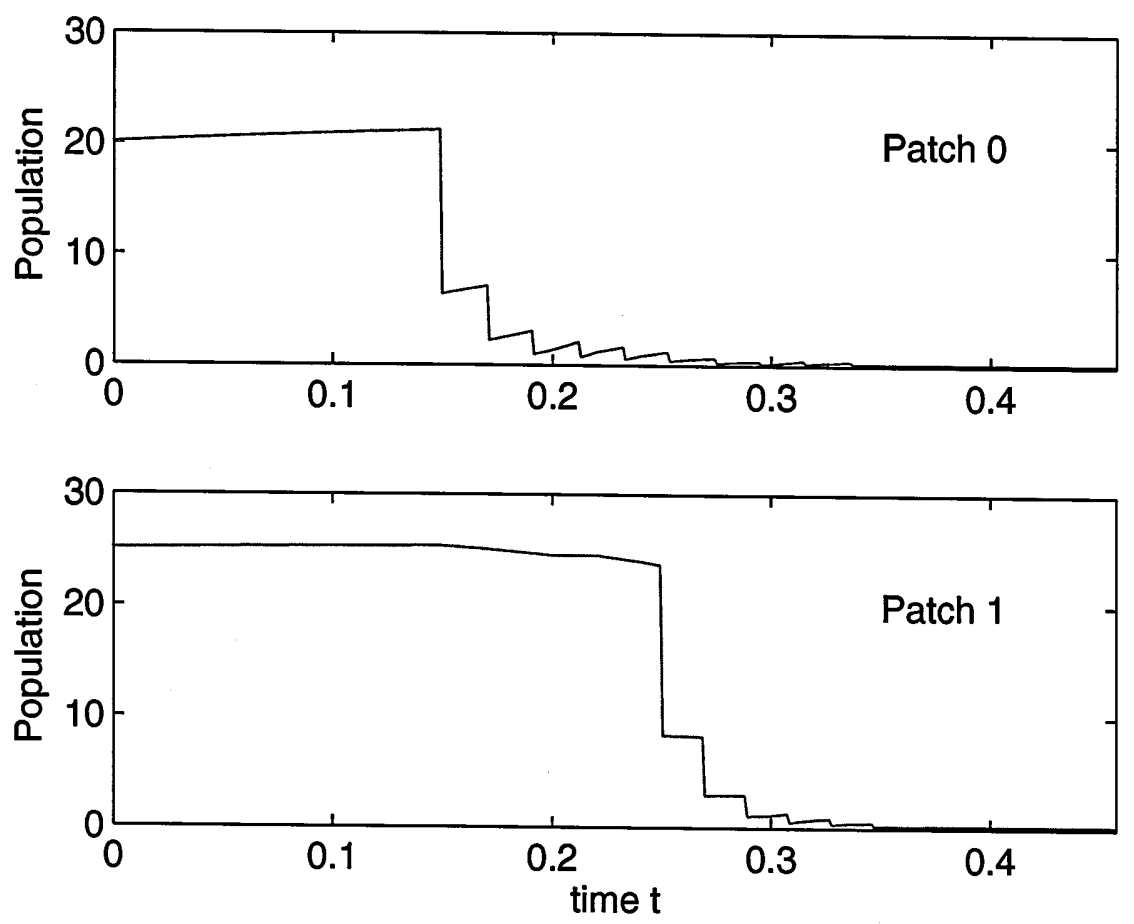

Figure 6.6: Culling when both birth functions are Allee. Model and culling parameters are stated in section 6.8 .

culling regime parameters are: $t_{0}=0.15, \epsilon_{0}=0.3, T_{0}=0.207, t_{1}=0.25, \epsilon_{1}=0.35$, $T_{1}=0.0193$. Note that the inter-cull times $\left(T_{0}\right.$ and $\left.T_{1}\right)$ are chosen to satisfy $(6.148)$, thereby guaranteeing eradication. Note further that we stop culling when the combined populations have, for at least $\tau$ consecutive time units, been kept below a level small enough for extinction to become inevitable with no further culling (corollary 5.1 ensures there is a minimum viable combined population). Thus we perform only 10 culls on patch 0 and 6 culls on patch 1 with no further culling required.

In figure 6.7 we plot the populations on both patches when the birth functions are Nicholson and culling does not occur frequently enough for eradication to be guaranteed by our results. From our particular choices of $\epsilon_{k}$ and $T_{k}$ we see that the culling regimes appear to maintain the average populations above the levels at which they would remain naturally, that is, in the absence of culling. The birth functions are: $b_{0}\left(w_{0}\right)=25 w_{0} e^{-w_{0}}$ and $b_{1}\left(w_{1}\right)=25 w_{1} e^{-w_{1}}$. The initial data is $\left(w_{0}(t), w_{1}(t)\right)=(5,5)$ for $t \in[-\tau, 0]$ and the delay $\tau=1$. Also $A=F=1.1$ and $B=C=D=E=$ $G=H=1$. (Hence $\theta_{3}=65.5075$.) The culling regime parameters are: $t_{0}=t_{1}=10$, $\epsilon_{0}=\epsilon_{1}=0.2951$, and $T_{0}=T_{1}=5.8$. Thus the patches and regimes are identical for this particular example.

An analogous observation (that "infrequent" culling can benefit a pest) was made in the case of a pest inhabiting a single patch in figure 3.3 (bottom right) in section 3.5. That observation for a pest on a single patch motivated us to prove in chapter 4 that, for a special type of birth function, which we called a step birth function, then infrequent culling can increase the mean population. We anticipate that some of the 

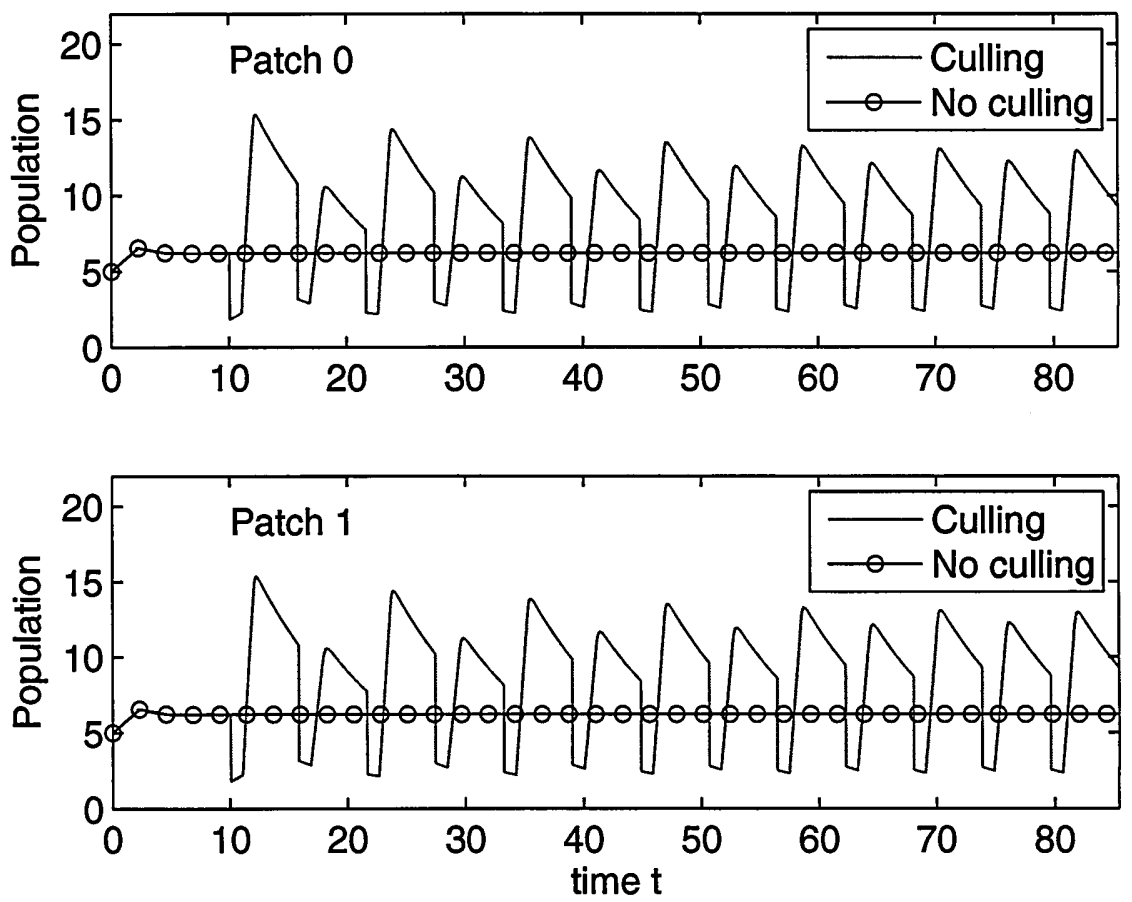

Figure 6.7: Perversity: infrequent culling can benefit a pest when both birth functions are Nicholson. See last two paragraphs of section 6.8 .

methods of chapter 4 may be adapted to prove that infrequent culling can increase the mean populations on both patches in the two patch model of the current chapter. In adapting the methods of chapter 4 to that end, we could begin by considering identical patches with identical initial data and then studying minor perturbations from these conditions.

\subsection{Discussion}

We have considered adult impulsive culling regimes in a model for a pest with a life cycle consisting of two stages (juvenile and adult) where the pest lives on two patches between which migration may occur. On either patch, the birth function was allowed to be any of three different kinds, namely linear, Nicholson, or Allee. We saw that there are circumstances in which, if culling occurs on only one of the patches, then the pest will not be eradicated on either patch. We concluded that successful control strategies will sometimes require culling simultaneously on both patches. Conditions on simultaneous culling regimes ensuring eradication on both patches were found. The regimes were chosen to be essentially independent, except in their simultaneity, giving individual freedom to whoever is in charge of a particular patch in terms of their pest control decisions.

An analysis of the conditions ensuring our simultaneous culling regimes are suc- 
cessful revealed that, as long as each cull has some effect, no matter how insignificant, then pest eradication will occur if consecutive culls on patch $k(k=0,1)$ are carried out sufficiently close in time. Also, when both birth functions are of Allee type, we used a result in chapter 5 to construct explicit finite upper bounds for the number of culls needed on each patch to guarantee eradication. Such a discovery helps us to believe that real-world pest control may be achieved by only a finite number of pesticide applications but should not be used as an excuse to believe that control is always attainable if only we apply enough pesticides. As discussed in section 2.3, decisions to apply pesticides, especially on a large scale, should be made with great caution.

Simulations corroborated our results. A simulation with Nicholson birth functions on both patches also suggested that culling infrequently can maintain the average populations on the patches above the levels at which they would remain naturally. Future research could involve proving analytically that infrequent culling on both patches can indeed increase the average pest populations, perhaps by adapting the methods of chapter 4 . Other future research could involve generalising our pest control work to three or more patches. Constructing successful independent culling regimes on three or more patches could require formidable algebra, however. 


\section{Part II}

\section{Disease control}


MATERIAL REDACTED AT REQUEST OF UNIVERSITY 


\section{Chapter 7}

\section{Introduction to disease control}

"Thinking is the most unhealthy thing in the world, and people die of it just as they die of any other disease."

Oscar Wilde (1856 - 1900)

\subsection{Mathematical epidemiology}

Mathematical epidemiology is the study of the spread of infectious diseases through the formulation and analysis of mathematical models. It is widely believed to have been initiated by Daniel Bernoulli in 1760 when he used a mathematical model to evaluate the effectiveness of the technique of variolation against smallpox, with the aim of influencing public health policy [52]. However, the subject did not really become established until the early twentieth century (p. 7, [47]) when investigations by, for example, Hamer [84], Ross [121], and Kermack and McKendrick [93] began to provide a firm theoretical framework for the study of observed patterns. An explosion in the literature has been ongoing since the mid 1950s, with the first edition of a now classic text by Bailey [51] representing an important landmark. Good introductions to the subject, including comments on its history and development, may be found in [47], [51], and [55].

Models in mathematical epidemiology should be referred to as epidemiological models. Unfortunately many authors call them epidemic models instead [76, 78, 99] even though the term "epidemic" has its own specific meaning. Not all authors agree on what this specific meaning is, but there seems to be a general consensus that such a meaning exists. One of the more precise definitions is given by Murray (p. 612, [112]). If a particular disease is introduced into a population by the arrival of $I(0)$ infected individuals, then an epidemic occurs (according to Murray) if at some point after the introduction, more than $I(0)$ individuals are infected. In essence, an epidemic for Murray is an outbreak that must get worse before it gets better. Of course, by this definition, an epidemic will occur if there is initially one infected individual and no more than two individuals infected at any future time. But these are hardly the kinds of figures most people would associate with the term "epidemic". Most people 
would think of an epidemic as a sudden outbreak, rapidly infecting a large number of individuals. Therefore, to avoid confusion, some authors attempt to translate this intuitive definition into more mathematical language. For example, Newman states that epidemics are "outbreaks that affect a non-zero fraction of the population in the limit of large system size" [113]. Hethcote tries even harder to avoid confusion by claiming that "epidemic models are used to describe rapid outbreaks that occur in less than one year"(p. 602, [87]). But he gives no indication as to why he considers one year to be the maximum permissible time span for an epidemic. In this thesis, we will define an epidemic to simply be a sudden rapid outbreak of a disease.

Inconsistencies in language have arisen from the eruption of literature in mathematical epidemiology in recent decades, including the emergence since the 1980s of a whole new field - the stochastic spread of diseases across networks of social contacts $[72,90,91,113,118,126]$. Perhaps in time, when the subject has acquired some maturity and a text is written with sufficient clarity and impact to become standard, then a standard language will be adopted.

When a new disease does appear in a population, there need not be an epidemic. The disease may die out or it may persist indefinitely but without ever spreading rapidly. A disease which persists indefinitely in a population may be deemed endemic. If a disease spreads after its initial introduction into a population, then it can be said to have invaded successfully.

\subsection{Types of model}

To derive an epidemiological model for a given population and a given disease, we generally begin by dividing the population into classes where the individuals in each class have the same disease status. The number of classes will depend upon the behaviour of the disease. The simplest example is when the population is split into: susceptibles $(S)$, namely individuals who do not have the disease but would catch it if they came into proper contact with a diseased individual; and infectives $(I)$, those who have the disease and can spread it.

A model is typically named after the order of stages through which an individual can pass. Thus, if a susceptible can become an infective, but an infective remains an infective until dying because the disease is incurable, then we would have a susceptible-infective or SI model. In an SI model, a susceptible does not have to become infected. It is called an SI model because a susceptible can become infected. A disease for which an SI model may be appropriate is herpes [54].

Another example is the SIR model. Here an individual may be either susceptible, infective, or removed $(R)$. Removed individuals are those who do not have the disease and cannot catch it, which could be because they are naturally immune, or have had the disease and acquired natural immunity in recovering from it (as may happen with some viral infections such as measles or rubella [98]), or they have been vaccinated.

In an SIR model, a susceptible will either remain susceptible until dying or will become infective, an infective will either remain infective until dying (possibly from the infection itself) or will recover, and a recovered individual will remain recovered until dying (and therefore be removed from disease circulation) because recovery is assumed to confer permanent immunity. We can summarise the information in the last sentence by using arrows to indicate the direction in which individuals may travel 
through the disease classes: $\mathrm{S}->\mathrm{I}->\mathrm{R}$. This is a practice often employed in the literature. Flow diagrams are also commonly used containing arrows and information on rates of change.

If recovery confers only temporary immunity, then a recovered individual may become susceptible again and may therefore subsequently become re-infected. We can again use arrows to summarise the disease flow: $\mathrm{S}->\mathrm{I}->\mathrm{R}->\mathrm{S}$. In this case, we have an SIRS model. An exposed class $(E)$, containing individuals who have become infected but are not yet infectious because the infectious agent is incubating, is frequently included in epidemiological models, giving rise to SEIR and SEIRS models. Other possibilities are the SIS and SEIS models, which can arise if recovery confers no immunity whatsoever, not even temporary.

Epidemiological models can be made more complex by incorporating features such as age-structure [87] or differences between the sexes [100]. Models can also be either stochastic or deterministic. Stochastic models have certain modelling advantages - individuals can be treated as discrete, for example, which becomes important when a population is small. However, stochastic models are typically much less tractable mathematically [51]. The three main models to be considered in this thesis will all be deterministic SIR models.

\subsection{Frequent assumptions}

Once we have decided which type of model is appropriate, we must gain some understanding of how often susceptibles come into contact with infectives. It is intuitively obvious that if a susceptible is more likely to come into contact with an infective, then they will be more likely to become infected. Such intuition gives rise to the wellknown principle of mass action (p. 7, [47]), also called the law of mass action [67], in which the net rate of spread of infection is assumed to be proportional to the product of the density of susceptible individuals and the density of infective individuals. In essence, a disease will spread more quickly if there are more individuals that can catch it and more that can spread it.

It is common to combine the mass action principle with an assumption of homogeneous mixing or homogeneity. Homogeneous mixing may be stated in these terms: for any particular individual $A$ and any other two individuals from the entire population, namely $B$ and $C$, then $A$ has the same chance of coming into contact with $B$ per unit time as $A$ has of coming into contact with $C$ per unit time, regardless of the disease status of $A, B$, or $C$. Such homogeneous mixing is not completely realistic of course but without such an assumption, one cannot always readily estimate the rate of new cases. The rate of new cases is called the incidence. Homogeneous mixing is frequently assumed in the literature $[60,76,98]$ but it is seldom stated in an explicit form.

A consequence of homogeneous mixing is that all individuals have the same average number of contacts $\beta$ per unit time. This contact rate $\beta$ is sometimes assumed to be constant or it may be written as a function of the total population, as we discover in chapter 8 . Given an assumption of homogeneity and an expression for the contact rate, we can calculate the incidence. But a number of other choices must still be made before a model can be derived - we must choose a suitable birth rate; we must be aware if foetuses can be infected and, if they can, at what rate (the passing of infection from mother to unborn child in the womb is called vertical transmission); we must choose a recovery rate if recovery is possible; we must choose appropriate 


\begin{tabular}{|l|l|l|}
\hline Cause of death & Deaths 1993 & Deaths 2002 \\
\hline Lower respiratory infections & 4.1 million & 3.9 million \\
HIV/AIDS & 0.7 million & 2.8 million \\
Diarrhoeal diseases & 3.0 million & 1.8 million \\
Tuberculosis (TB) & 2.7 million & 1.6 million \\
Malaria & 2.0 million & 1.3 million \\
Measles & 1.1 million & 0.6 million \\
Pertussis & 0.36 million & 0.29 million \\
Tetanus & 0.15 million & 0.21 million \\
Meningitis & 0.25 million & 0.17 million \\
Syphilis & 0.19 million & 0.16 million \\
Hepatitis B & 0.93 million & 0.10 million \\
Tropical diseases & 0.53 million & 0.13 million \\
\hline
\end{tabular}

Table 7.1: Global mortality due to infectious diseases. Data for 1993 obtained from the 1995 World Health Report [32] and data for 2002 obtained from the 2004 World Health Report [33]. In the table: lower respiratory infections include various pneumonias, influenzas and acute bronchitis; diarrhoeal diseases are caused by many different organisms and include cholera and botulism; and tropical diseases include Chagas disease, dengue fever, lymphatic filariasis, leishmaniasis, onchocerciasis, schistosomiasis and trypanosomiasis.

"natural" and disease death rates (a "natural" death is one not caused by the disease being modelled); and so on. All of the rates just mentioned may be estimated from real-world data when it is available. However, a significant proportion of papers in mathematical epidemiology do not use real data. Instead they establish theoretical results from which practical decisions could be made if combined with real-world data. It will become clear how to specifically go about deriving a model in chapter 8 when we explicitly derive an SIR model.

\subsection{Vaccination strategies}

Epidemiological models attain their true practical value when vaccination strategies are incorporated into them. When this is done, we may calculate how to bring a disease under control. Ultimately the goal should be to save lives and reduce suffering. This was the intention of Daniel Bernoulli when he initiated the subject in 1760. Although the mortality for many infectious diseases has fallen dramatically in recent decades due to vaccination programs, a fearful toll continues to be exacted every year (see table 7.1). What is most tragic about many of these deaths is that they are preventable, an issue we discuss in section 7.6 below.

There are two main types of vaccination strategy. Before describing them we note that a vaccination is successful if it confers immunity (temporary or permanent) to the disease we are seeking to control. It is often assumed for analytical simplicity that vaccination confers permanent immunity, even though, in practice, this may not be the case. Many diseases are protected against by two separate vaccinations - one shortly after birth and the other some years later, for example measles, mumps, or rubella [31]. Temporary immunity and repeated vaccination has received attention 
from d'Onofrio, for example [69]. In this thesis, we will, for simplicity, assume that vaccination, if successful, confers permanent immunity.

The most commonly implemented vaccination strategy is the constant vaccination strategy (CVS) [123]. A CVS involves or requires the successful vaccination of a fixed proportion of all new borns (or infants, if the vaccination is unsuitable for babies). Thus to incorporate a CVS into an epidemiological model, it is usual to assume that a constant proportion of susceptibles are effectively born into a removed class, by virtue of their immunity by vaccination.

The second main vaccination method is the pulse vaccination strategy (PVS) [123]. This is a sequence of vaccination episodes called pulses. The pulses are of short duration compared to the time elapsing between consecutive pulses. Each pulse requires the successful vaccination of a fixed proportion of the entire susceptible population, not just new borns. In epidemiological models, the sequence of pulses is typically assumed to be of unlimited length and each pulse is assumed to occur instantaneously because, in relation to the time between pulses (the inter-pulse time) or the dynamics of the disease [71], a pulse will be of very short duration. Notice the conceptual similarity between using vaccination pulses to control a disease and using impulsive culls to control a pest.

It is plausible that resonance, the excitation of oscillations by external forcing, will occur in real-world epidemiological systems. For example, disease levels amongst school children fluctuate between term time and holidays, and vaccination pulses are a form of external forcing. These issues are discussed in a paper by Choisy et al [61], who show by simulation in an SEIR model that increasing the pulse frequency (that is, decreasing the inter-pulse time) can have perverse effects such as increasing the number of infectives.

It is possible to impose both constant and pulse vaccination strategies on a population at the same time, producing a "mixed" vaccination strategy (MVS).

A disease may be eradicated absolutely or relatively. Absolute eradication requires the number of infectives to tend to zero as time tends to infinity. Relative eradication requires the proportion of infectives to tend to zero as time tends to infinity. We will consider a vaccination strategy to be absolutely successful if it ensures absolute eradication of the disease. It will be relatively successful if it ensures relative eradication of the disease.

Implementation of a constant vaccination strategy (CVS) may be difficult in a region lacking medical infrastructure. Newborns cannot be vaccinated if there is nowhere to take them to be vaccinated. In such a situation, a pulse vaccination strategy (PVS), in which a team of medics goes out and pulse vaccinates a region, is a better option. Moreover, a PVS may require lower coverage than a CVS to bring the disease under control - the proportion that must be vaccinated in each pulse may not need to be as high as the proportion of newborns that must be vaccinated in a CVS, provided the pulses occur frequently enough [123]. Furthermore, there may be situations in which, with a PVS, we may not, in the long run, need to vaccinate as many individuals in total [70].

There have been numerous real-world successes with constant vaccination programs since the first government-backed programs of the nineteenth century. Probably the most striking success so far was the global eradication by 1979 of smallpox [28], which hitherto killed many millions and even helped to bring down empires [104]. Pulse vaccination strategies are newer, with major programs attaining notable success in the control of measles and poliomyelitis in Central and South America 
in the early 1990s [123]. An ongoing initiative called Pulse Polio is helping to bring poliomyelitis under control in India [85]. Today, vaccinations are a massive component of public health control. A newborn baby in the developed world can now expect (if we suppose they are capable of expecting anything) to have had at least five injections before they utter their first intelligible word [31].

\subsection{Disease control problems in this thesis}

In the next three chapters we will examine three epidemiological models, which are connected only in so far as they explore matters hitherto unexplored.

In chapter 8 , we will begin by noting that: human populations have been growing steadily for decades and this trend is predicted to continue in many regions; higher populations have been linked to higher contact rates; and within many populations there circulate diseases with low mortality. These observations motivate us to consider an SIR model for a non-fatal disease in which the total population grows exponentially and the contact rate grows with the population. For this model, we will discover that a pulse vaccination strategy has no long-term control effect at all, whereas a constant vaccination strategy reduces the long-term proportion of infectives. Simulations will corroborate our results and conclusions will be drawn on the implications for public health policies.

Next, in chapter 9, we will examine a model for a disease with SIR-type dynamics for a population living on $n$ patches between any pair of which migration is allowed. We will suppose that a pulse vaccination strategy (PVS) is carried out on each patch. Conditions will be derived on each PVS such that the disease will be eradicated on all patches. The PVS on one patch will be essentially independent of the PVS on the other patches except in so far as they will all be performed simultaneously. This independence will be of practical value when we bear in mind that the patches may represent regions or countries with autonomous public health authorities, which may make individual decisions about the days appropriate for a vaccination pulse to occur in their own region or country.

Finally, in chapter 10, we will notice that few epidemiological models for mammalian species in the literature have included a gestation delay, so in our final model we will incorporate such a delay. The disease will have SIR-type dynamics. After proving that the disease can remain endemic, successful pulse and constant vaccination strategies will be constructed. We will also discuss the related field of insect pathogen dynamics, observing in particular that certain insect pathogen models may reduce to mammalian epidemiological models.

\subsection{Hindrances to disease control}

Just as we recognised in section 2.3 that the application of pesticides should be placed within an environmental context, so it is sensible to view disease control within a socio-economic and political milieu.

Major factors contributing to the spread of infectious diseases, and hindering efforts to control them, include war, poverty, dictatorships, and corruption. For example, civil conflict in the Darfur region of Sudan since 2003 has led to a massive displacement of people. Millions have fled their homes, the majority ending up in 
camps for displaced persons or refugees (p. 16, [37]). In many such camps overcrowded and unsanitary conditions have promoted the spread of infectious diseases which have killed thousands [38]. The Sudanese government has made access to these unfortunate people very difficult, preferring to persecute them by orchestrating and participating in war crimes and crimes against humanity according to a UN report of 2007 (p. 25, [37]). Currently (January 2009) a humanitarian crisis rages in Darfur and has spread into neighbouring Chad as hundreds of thousands of refugees have fled there (p. 16, [37]). Implementing vaccination programs under such conditions is a difficult and dangerous task. Indeed many aid workers in Darfur have been killed or attacked since the conflict began (p. 14, [37]). There are circumstances in which disease control is as much an issue of international diplomacy, law, and even military intervention as it is of vaccinating individuals.

Another awful situation is the plight of Zimbabwe. Years of incompetent and ruthless dictatorship have led to hyperinflation and extreme poverty. In the period from August to December 2008, the collapse of health and sanitation systems exacerbated an outbreak of cholera [42]. Instead of inviting international aid, senior Zimbabwean politicians denied there was a serious problem. Meanwhile hundreds of people were dying [40]. Although assistance was finally requested in December 2008 [42] and aid workers from Medicins Sans Frontieres were able to bring in emergency cholera units [41], the disease had by then spread to neighbouring countries [42] as infected people escaped Zimbabwe in search of treatment and a better life. The example of Zimbabwe tells us that the effective control of diseases, including the prevention of outbreaks, is intimately related to the existence of basic economic infrastructure. But the existence and construction (or re-construction) of such infrastructure is heavily dependent on political stability, and ensuring the political stability of countries such as Zimbabwe is no trivial matter, even for the international community.

Corruption is most common in the poorest countries [18] but it is in such countries that infectious diseases are most likely to be endemic. When corruption takes away resources from health care in poor nations, unnecessary deaths result [43].

Efforts to control diseases are also hindered when senior political figures make grand statements about medical matters which they are not qualified to make. The claim by leading figures in South Africa that poverty, and not HIV, is a cause of AIDS has served only to retard the national response to the AIDS pandemic that is ravaging the country [36]. Another factor that obstructs attempts to control HIV/AIDS is the social stigma attached to it because it is a sexually transmitted disease [20]. To adequately tackle a disease such as HIV/AIDS in a country such as South Africa, it is clear that a broad range of social, economic, and political issues must be addressed. Education to encourage safe sex and discourage the social stigma of AIDS, further investment in health care systems, and increased access to health care, are all of paramount importance.

Of course it is not only in developing countries that disease control is hampered by social attitudes. In the UK, selective and misleading media coverage of a purported link, made in 1998 [137], between the MMR (Measles, Mumps, and Rubella) vaccine and autism has led to decreased parental confidence in the vaccine and a reduction in the percentage of children receiving it [103]. The link has been discredited in various reports [66] but public mistrust and reduced levels of vaccination have continued for years after the initial controversy [103], and in 2008, for the first time in 14 years, measles was declared endemic in the UK (see [132] and see figure 7.6 for a picture of a measles virion). The media in the UK has damaged public confidence in a safe vaccine and therefore has a responsibility to restore public confidence. But even 10 


\section{MATERIAL REDACTED AT REQUEST OF UNIVERSITY}

years after the initial controversy, such confidence has yet to be restored.

Mistrust in vaccines can arise for reasons other than a sensationalist media. Conservative religious leaders, for religious or political purposes, have been known to discourage their followers from receiving vaccinations. Such action led, for example, to the suspension for several months in 2003, of the oral polio vaccine program in northern Nigeria, consequently allowing a revival of the disease [62].

The control of diseases is a multi-faceted and complex issue. The mathematical calculation of successful vaccination strategies plays an important role but it is critical to bear in mind that vaccination programs, or other medical initiatives, cannot be introduced into a region or country unless a diverse range of socio-economic and political conditions are satisfied, not the least of which include trust, freedom from corruption, and political stability. 


\title{
Chapter 8
}

\section{Population-dependent contact rates in an SIR model}

\author{
"Population, when unchecked, increases in geometrical progression of such a nature \\ as to double itself every twenty-five years." \\ Thomas Malthus (1766 - 1834)
}

\subsection{Introduction}

There has been a tendency for human populations to grow in recent decades and centuries, and this trend is predicted to continue in many regions of the world for the foreseeable future (see table 8.1 and references $[34,105]$ ). When the population in a human settlement grows, the settlement does not usually expand fast enough geographically to prevent the population density from increasing. After all, it is easier to build new houses near existing civic infrastructure - schools, hospitals, shops - than it is to build new houses and new infrastructure to service their future occupants.

Studies have shown that contact rates in human populations are higher when the population itself is higher [46]. Intuitively we can reason that if a population increases, then the population density may increase, which may cause any particular individual to have more contacts in their daily life, since it will be more crowded in shops, pubs, town and city centres, on public transport, and so on. Evidence for this line of reasoning comes from Arita et al's study of smallpox eradication [50], which contains data linking a higher population density with increased disease incidence (recall that the incidence is the rate of new cases), suggesting a higher contact rate since the disease was usually spread by close person-person contact [28].

Within many growing populations circulate diseases with low fatality rates, such as measles, varicella (chickenpox), mumps, and pertussis (whooping cough) [30]. As a rough approximation, made for mathematical simplicity, we could assume that individuals infected with such a disease have the same mortality rate as those not infected, that is, the "natural" mortality rate. It is not uncommon to make such a simplifying assumption $[44,123]$. 


\begin{tabular}{|l|l|l|l|l|l|l|l|l|}
\hline Region & 1750 & 1800 & 1850 & 1900 & 1950 & 1999 & 2050 & 2150 \\
\hline World & 791 & 978 & 1,262 & 1,650 & 2,521 & 5,978 & 8,909 & 9,746 \\
Africa & 106 & 107 & 111 & 133 & 221 & 767 & 1,766 & 2,308 \\
Asia & 502 & 635 & 809 & 947 & 1,402 & 3,634 & 5,268 & 5,561 \\
Europe & 163 & 203 & 276 & 408 & 547 & 729 & 628 & 517 \\
Latin America and & 16 & 24 & 38 & 74 & 167 & 511 & 809 & 912 \\
the Caribbean & & & & & & & & \\
Northern America & 2 & 7 & 26 & 82 & 172 & 307 & 392 & 398 \\
Oceania & 2 & 2 & 2 & 6 & 13 & 30 & 46 & 51 \\
\hline
\end{tabular}

Table 8.1: World historical and predicted populations (in millions). Here Northern America comprises the northern countries and territories of North America: Canada, the United States, Greenland, Bermuda, and St. Pierre and Miquelon. Latin America comprises Middle America (Mexico, the nations of Central America, and the Caribbean) and South America. The data is taken from a UN report of 2004 [3].

Note that whilst measles and pertussis, for example, have low mortality rates, thousands of deaths still occur every year (see table 7.1) because of the very large number of infections. Moreover the impact of disease is not restricted to diseasecaused deaths. Mild infections can adversely affect a country's economy by causing children to miss days at school and adults to miss days at work. There is value in controlling the spread of such infections.

Many viral infections tend to confer permanent immunity through recovery and some have relatively short incubation periods (p. 31, [47]). To model the spread of an infectious disease with a short incubation period and from which recovery confers permanent immunity, it is common to assume SIR-type dynamics [123], where $S, I$, and $R$ denote susceptibles, infectives, and removeds respectively. The terms susceptible, infective, and removed are defined in section 7.2.

In view of all of our comments so far, there is value in examining the circulation of a disease, modelled as non-fatal with SIR-type dynamics, in a growing population, in which the contact rate is allowed to grow with the population. Therefore, our purpose in this chapter will be to explore such a model, both in the absence of vaccination and with different types of vaccination strategy imposed on the model.

The format of this chapter is as follows. In section 8.1.1, we discuss contact rates which grow with the population. In section 8.2 we derive an SIR model in an exponentially growing population where the contact rate is either a linear function of the population or is proportional to a power $v$ of the population where $0<v<1$. In section 8.3 we consider how the susceptible population changes in our model as time passes. We use our understanding of the behaviour of the susceptible population to investigate, in section 8.4 , the influence of vaccination strategies. Simulations are included in section 8.5 and a discussion concludes the chapter in section 8.6.

\subsubsection{Contact rates}

Suppose an infectious disease is circulating in a population. Assume there are $N(t)$ individuals at time $t$. Suppose any particular individual must be either susceptible, infective, or removed with respect to the disease at any given time. Let there be 
$S(t)$ susceptibles, $I(t)$ infectives, and $R(t)$ removeds at time $t$. Notice that $N(t)=$ $S(t)+I(t)+R(t)$. Assume, for simplicity, that all individuals have the same average number of contacts per unit time (unit time could be one day, for example). Let this individual average contact rate be a function of the total population size and call it $\beta(N(t))$. We understand a "contact" to be adequate for disease transmission if it is between an infective and a susceptible. Assume homogeneous mixing in the sense described in section 7.3.

How many new cases might we expect in a short time interval $[t, t+d t]$ ? By our assumptions, we see that one infective will have, on average, $\beta(N(t)) d t$ contacts in this time interval. But one infective could come into contact with any of the $N(t)-1$ other individuals in the population, of whom $S(t)$ are susceptibles. Thus, by our assumption of homogeneous mixing, a proportion $\frac{S(t)}{N(t)-1}$ of the $\beta(N(t)) d t$ contacts of one infective will be with susceptibles, so the number of disease-transmitting contacts due to one infective will be $\beta(N(t)) \frac{S(t)}{N(t)-1} d t$. But then the total number of new cases due to all $I(t)$ infectives will be $\beta(N(t)) \frac{S(t) I(t)}{N(t)-1} d t$. Strictly speaking, this expression is only accurate (assuming our modelling assumptions are correct of course) in the limit as $d t \rightarrow 0$ since $\beta(N(t)), S(t), I(t)$, and $N(t)$ may all vary when $t \in[t, t+d t]$. Said differently, if $d t$ is very small, then, for example, $I(t)$ is a good approximation for the number of infectives on the entire interval $[t, t+d t]$ but otherwise it may not be. The expression $\beta(N(t)) \frac{S(t) I(t)}{N(t)-1}$ is the rate of new cases and is called an incidence function. It is usual to assume, and shall be assumed by us, that $N(t)$ is large enough to allow $\beta(N(t)) \frac{S(t) I(t)}{N(t)-1} d t$ to be accurately replaced by $\beta(N(t)) \frac{S(t) I(t)}{N(t)} d t$.

The dependence of the contact rate on the population size has been explored by letting the contact rate $\beta(N(t))$ equal $\Omega(N(t))^{v}$ for constants $\Omega$ and $v$ with $\Omega>0$ and $0 \leq v \leq 1$ (pp. 305-306, [47])( [46]). The special case $v=0$ corresponds to a constant contact rate and yields the standard incidence function [87], also called true mass action incidence [65]. The special case $v=1$ corresponds to linear dependence of the contact rate on the population and yields the simple mass action incidence function [98], also called pseudo mass action [65]. The case $0<v<1$ lies between the extremes $v=$ 0 and $v=1$, so the incidence function it yields may be deemed intermediate mass action to signify that it lies between true and pseudo mass action. Notice, however, that confusion has arisen in the labelling of incidence functions in recent years due to the explosion in the literature of mathematical epidemiology (see [102] for further comments on this matter).

Data for five human diseases in communities with population sizes from 1,000 to 400,000 implied that $v$ is between 0.03 and 0.07 (pp. 305-306, [47])( [46]). Since this is positive, there is value in exploring a model with contact rate equal to $\Omega(N(t))^{v}$ for $0<v \leq 1$, as we do in this chapter. But since the range $0.03<v<0.07$ is quite close to zero, there would also be value in analysing a model with a constant contact rate (corresponding to $v=0$ ). Busenberg and van den Driessche have performed such an analysis in a relatively simple model [60], although they do not consider the impact of vaccination strategies.

The influence of a variable population size on the dynamics of a disease has already received attention $[60,76,98,106]$ but the combined influence of a variable population size and a contact rate that varies with population size is less well explored [70,140]. The combined influence in an SIR model of an exponentially growing population and a contact rate proportional to a power $v$ of the population (with $0<v<1$ ) has not, to our knowledge, been studied. We will study it in this chapter. 


\subsection{An SIR model}

Make the assumptions in the first paragraph of section 8.1.1. Assume additionally that the disease may be modelled (perhaps only approximately) as non-fatal. Define the following model parameters:

- $\lambda=$ per capita birth rate

- $\mu=$ per capita death rate for all classes (no disease fatalities)

- $\gamma=$ per capita recovery rate of infectives

All of these parameters are assumed to be positive. Assume all new borns are susceptible and that recovery confers permanent immunity. Also, in view of our comments on growing populations in section 8.1, assume that the birth rate $\lambda$ is bigger than the death rate $\mu$. By considering the changes in the numbers of susceptibles, infectives, and removeds in a short time interval $[t, t+d t]$, and letting $d t \rightarrow 0$ whilst noticing, for example, that $\lim _{d t \rightarrow 0}\left(\frac{S(t+d t)-S(t)}{d t}\right)=\frac{d S(t)}{d t}$, our hypotheses lead to the following model:

$$
\begin{aligned}
\frac{d S}{d t} & =\lambda N-\beta(N) \frac{S I}{N}-\mu S \\
\frac{d I}{d t} & =\beta(N) \frac{S I}{N}-(\gamma+\mu) I \\
\frac{d R}{d t} & =\gamma I-\mu R,
\end{aligned}
$$

where we use the shorthand $S, I, R$, and $N$ for $S(t), I(t), R(t)$, and $N(t)$ respectively.

Equations (8.1) to (8.3) are our epidemic model, and we will analyse it for two specific contact rates mentioned in section 8.1.1, namely $\beta(N)=\Omega N^{v}$ ( $\Omega$ constant, $v$ constant, $\Omega>0$ ) for $v=1$ or $0<v<1$. To make these different contact rates more distinguishable, we replace $\Omega$ by $\eta$ in the case $v=1$. Thus the two cases to be explored are:

1) Contact rate $\beta(N)=\eta N$, for $\eta$ constant, $\eta>0$. This yields the simple mass action incidence function $\beta(N) \frac{S I}{N}=\eta S I$, so the case will be referred to as simple mass action incidence.

2) Contact rate $\beta(N)=\Omega N^{v}$, for constants $\Omega, v$ where $\Omega>0$ and $0<v<1$. This yields the intermediate mass action incidence function $\beta(N) \frac{S I}{N}=\Omega S I N^{v-1}$, so the case will be referred to as intermediate mass action incidence.

If we incorporate a constant vaccination strategy (CVS) into the model, where a constant proportion $\pi$ of all newborns is vaccinated successfully and on a continuous basis, then the model becomes:

$$
\begin{aligned}
\frac{d S}{d t} & =(1-\pi) \lambda N-\beta(N) \frac{S I}{N}-\mu S \\
\frac{d I}{d t} & =\beta(N) \frac{S I}{N}-(\gamma+\mu) I \\
\frac{d R}{d t} & =\gamma I-\mu R+\pi \lambda N .
\end{aligned}
$$


Since $\pi$ is a proportion, we have $0 \leq \pi \leq 1$. If $\pi=0$, then no new borns are vaccinated successfully and the model reduces to the model with no vaccinations. If $\pi=1$, then all new borns are vaccinated successfully, and it becomes trivial to show that the disease must inevitably die out. Assume for the rest of this paper, then, that $0 \leq \pi<1$.

Now suppose a pulse vaccination strategy (PVS) is incorporated into the model according to the following definition:

Definition 8.1. Pulses occur every $T>0$ time units, where $T$ is fixed. The first pulse occurs at time $t_{1}>0$ and, for $i \geq 2$, the $i$-th pulse occurs at time $t_{i}=t_{1}+(i-1) T$. Each pulse instantaneously transfers a fixed proportion $p>0$ of susceptibles to the removed class. Thus $S\left(t_{i}\right)=(1-p) S\left(t_{i}^{-}\right)$and $R\left(t_{i}\right)=R\left(t_{i}^{-}\right)+p S\left(t_{i}^{-}\right)$where $t_{i}^{-}$is the time "momentarily" before time $t_{i}$. Also $I\left(t_{i}\right)=I\left(t_{i}^{-}\right)$.

Hence, when there is a PVS, the model evolves between pulses in accordance with equations (8.1), (8.2), and (8.3), but at each pulse the numbers in each class are reset thus:

$$
S\left(t_{i}\right)=(1-p) S\left(t_{i}^{-}\right), \quad I\left(t_{i}\right)=I\left(t_{i}^{-}\right), \quad R\left(t_{i}\right)=R\left(t_{i}^{-}\right)+p S\left(t_{i}^{-}\right) .
$$

The model with a PVS therefore consists of a series of initial value problems, with a new problem beginning with each pulse. Assume that the pulses do not attain complete coverage (which would anyway be unrealistic for a real-world program), that is, assume $0 \leq p<1$. Let $q=1-p$.

If a CVS or PVS were carried out in a real population, the vaccination success rates would probably vary with time because not everybody would react to the vaccination in the desired fashion - some people may have an allergic reaction, for example, and others may refuse to be vaccinated. Variation in the vaccination success rates is unlikely to unsettle a public health authority as long as the success rates are kept above the minimum level needed to bring the disease under control.

For all of the models - without vaccination, with a CVS, or with a PVS - and letting the incidence function be either of the two under consideration, it is easy to deduce from standard results (p. 149, [131]) that, given initial data $S(0), I(0), R(0)$, a unique solution exists for $t>0$. It is also straightforward to deduce from known results (p. 81 , [128]) that if $S(0)>0, I(0)>0, R(0) \geq 0$, then $S(t)>0, I(t)>0, R(t)>0$ for $t>0$. This property is called strict positivity (the property would be non-strict if we had $S(t) \geq 0, I(t) \geq 0, R(t) \geq 0$ for $t>0)$. Henceforth assume that $S(0)>0, I(0)>0$, $R(0) \geq 0$. Necessarily, then, $N(0)>0$. In fact, since $S(0)$ and $I(0)$ represent initial populations and are assumed positive, then we should write $S(0) \geq 1, I(0) \geq 1$, and consequently $N(0) \geq 2$.

If we add together equations (8.1), (8.2), and (8.3), and bearing in mind that $S(t)+$ $I(t)+R(t)=N(t)$, we obtain:

$$
\frac{d N}{d t}=(\lambda-\mu) N
$$

which implies that

$$
N(t)=N(0) e^{(\lambda-\mu) t} \text { for } t>0,
$$

so the population will grow exponentially with time (recall that $\lambda>\mu$ ).

Equation (8.9) also holds for the model with a CVS (as we can see by adding together equations (8.4), (8.5), and (8.6)) and for the model with a PVS (because the 
model equations are the same between pulses as in the model with no vaccinations and each instantaneous pulse does not alter the size of the total population).

Henceforth "no VS" will be understood to mean "no vaccination strategy", that is, a complete absence of vaccination. In subsequent sections if we refer to the "no VS models", we will mean "the model with no vaccinations and either simple or intermediate mass action". A similar comment applies to the "CVS models" and the "PVS models".

\subsection{Susceptible population}

In this section we consider the behaviour of the susceptible population in our models, both as a proportion of the total population and in terms of raw numbers.

Lemma 8.1. In all the models (no VS, CVS, or PVS), when the incidence function is either simple or intermediate mass action, we have $\frac{S(t)}{N(t)} \rightarrow 0$ as $t \rightarrow \infty$.

Proof. We consider the CVS models first (the no VS models are a special case obtained by setting $\pi=0$ ). We begin by noting that, when the incidence is simple mass action, then, for $t>0$ :

$$
\begin{aligned}
\frac{d}{d t}\left(\frac{I(t)}{N(t)}\right) & =\left(\eta N\left(\frac{S}{N}\right)-(\lambda+\gamma)\right)\left(\frac{I}{N}\right) \\
& >\left(\alpha N^{v}\left(\frac{S}{N}\right)-(\lambda+\gamma)\right)\left(\frac{I}{N}\right),
\end{aligned}
$$

where $\alpha=\min (\eta, \Omega)$ and where we use the facts from section 8.2 that $N(0) \geq 2>$ 1 , that $N(t)$ is growing for $t>0$, and that strict positivity holds. Also, when the incidence is intermediate mass action, we find for $t>0$ that:

$$
\begin{aligned}
\frac{d}{d t}\left(\frac{I(t)}{N(t)}\right) & =\left(\Omega N^{v}\left(\frac{S}{N}\right)-(\lambda+\gamma)\right)\left(\frac{I}{N}\right) \\
& \geq\left(\alpha N^{v}\left(\frac{S}{N}\right)-(\lambda+\gamma)\right)\left(\frac{I}{N}\right) .
\end{aligned}
$$

We want to show that $\frac{S(t)}{N(t)} \rightarrow 0$ as $t \rightarrow \infty$, or, equivalently, for any $\epsilon>0$, then for all $t$ big enough, $\frac{S(t)}{N(t)}<\epsilon$. Suppose for a contradiction that this is not true. Then, for some $\epsilon>0$, there is no time after which $\frac{S(t)}{N(t)}$ is always less than $\epsilon$. There will be a time $t^{*}$, then, arbitrarily large, such that $\frac{S\left(t^{*}\right)}{N\left(t^{*}\right)}>\epsilon$.

Now for a constant $\Delta>0$, what is the smallest that $\frac{S(t)}{N(t)}$ can be when $t \in\left[t^{*}-\Delta, t^{*}\right]$ given that $\frac{S\left(t^{*}\right)}{N\left(t^{*}\right)}>\epsilon$ ? To help answer this, observe that when the incidence is simple mass action then, for $t>0$ :

$$
\frac{d}{d t}\left(\frac{S(t)}{N(t)}\right)=\lambda(1-\pi)-(\eta I+\lambda)\left(\frac{S}{N}\right)
$$

and when the incidence is intermediate mass action then, for $t>0$ :

$$
\frac{d}{d t}\left(\frac{S(t)}{N(t)}\right)=\lambda(1-\pi)-\Omega N^{v}\left(\frac{S}{N}\right)\left(\frac{I}{N}\right)-\lambda\left(\frac{S}{N}\right) .
$$


Hence, by positivity, $\frac{d}{d t}\left(\frac{S(t)}{N(t)}\right)<\lambda$. It follows that a lower bound for $\frac{S(t)}{N(t)}$ when $t \in\left[t^{*}-\Delta, t^{*}\right]$ and $\frac{S\left(t^{*}\right)}{N\left(t^{*}\right)}>\epsilon$ is then $\epsilon-\lambda \Delta$. This is obvious geometrically but we shall give an analytic explanation. Suppose that $\epsilon-\lambda \Delta$ is not such a lower bound. Then for some $\bar{t} \in\left[t^{*}-\Delta, t^{*}\right]$, we must have $\frac{S(t)}{N(t)}<\epsilon-\lambda \Delta$. Now we can say that, since $\frac{d}{d t}\left(\frac{S(t)}{N(t)}\right)<\lambda$, then $\frac{S(t)}{N(t)} \leq x(t)$ for $t \geq \bar{t}$ where $x(\bar{t})=\epsilon-\lambda \Delta>\frac{S(\bar{t})}{N(\bar{t})}$ and $\frac{d x(t)}{d t}=\lambda$ for $t>\bar{t}$. Solving for $x(t)$ then yields:

$$
\frac{S(t)}{N(t)} \leq x(t)=\epsilon+\lambda(t-\bar{t}-\Delta) \text { for } t \geq \bar{t} .
$$

But then $\frac{S\left(t^{*}\right)}{N\left(t^{*}\right)} \leq \epsilon+\lambda\left(t^{*}-\bar{t}-\Delta\right) \leq \epsilon$ since $\bar{t} \in\left[t^{*}-\Delta, t^{*}\right]$. We have a contradiction, so $\epsilon-\lambda \Delta$ must be a lower bound for $\frac{S(t)}{N(t)}$ when $t \in\left[t^{*}-\Delta, t^{*}\right]$.

Choose $\Delta$ so that $\epsilon-\lambda \Delta \geq \frac{\epsilon}{2}$. This implies that $\Delta \leq \frac{\epsilon}{2 \lambda}$. Then, for $t \in\left[t^{*}-\Delta, t^{*}\right]$ we have $\frac{S(t)}{N(t)} \geq \frac{\epsilon}{2}$.

Since we can pick $t^{*}$ arbitrarily large, and since $N(t)$ grows exponentially, we can pick $t^{*}$ so that:

$$
\alpha\left(N\left(t^{*}-\Delta\right)\right)^{v}\left(\frac{\epsilon}{2}\right)>A,
$$

for any $A>0$. We will choose a specific $A$ in a moment.

Then, for $t \in\left[t^{*}-\Delta, t^{*}\right]$, we have, using (8.10), (8.11), and (8.15):

$$
\frac{d}{d t}\left(\frac{I(t)}{N(t)}\right)>(A-(\lambda+\gamma))\left(\frac{I}{N}\right) \text {. }
$$

This implies, since $\frac{I\left(t^{*}-\Delta\right)}{N\left(t^{*}-\Delta\right)}>0$ by strict positivity, that, for $t \in\left[t^{*}-\Delta, t^{*}\right]$ :

$$
\frac{I(t)}{N(t)} \geq\left(\frac{I\left(t^{*}-\Delta\right)}{N\left(t^{*}-\Delta\right)}\right) e^{(A-(\lambda+\gamma))\left(t-\left(t^{*}-\Delta\right)\right)}
$$

Since $\frac{I\left(t^{*}-\Delta\right)}{N\left(t^{*}-\Delta\right)}>0$, we can choose $A$ so that:

$$
\left(\frac{I\left(t^{*}-\Delta\right)}{N\left(t^{*}-\Delta\right)}\right) e^{(A-(\lambda+\gamma))\left(t^{*}-\left(t^{*}-\Delta\right)\right)}>1
$$

In other words, we can choose $A$ so that:

$$
\left(\frac{I\left(t^{*}-\Delta\right)}{N\left(t^{*}-\Delta\right)}\right) e^{(A-(\lambda+\gamma)) \Delta}>1
$$

It is not immediately apparent that an $A$ and $t^{*}$ can be found to satisfy (8.15) and (8.19) simultaneously, so we show that this is possible. Using (8.9), we find that (8.15) amounts to asking for:

$$
k_{1} e^{k_{2} t^{*}}>A
$$

where $k_{1}$ and $k_{2}$ are positive constants. (Specifically $k_{1}=\alpha(N(0))^{v} e^{-(\lambda-\mu) v \Delta}\left(\frac{\epsilon}{2}\right)$ and $k_{2}=(\lambda-\mu) v$.) 
Now by (8.10) and (8.11), and using positivity, we can say that $\frac{d}{d t}\left(\frac{I(t)}{N(t)}\right)>-(\lambda+$ $\gamma)\left(\frac{I(t)}{N(t)}\right)$ for $t>0$. It follows that $\frac{I(t)}{N(t)} \geq y(t)$ for $t \geq 0$ where $y(0)=\frac{I(0)}{N(0)}$ and $\frac{d y(t)}{d t}=-(\lambda+\gamma) y(t)$ for $t>0$. Solving for $y(t)$ yields:

$$
\frac{I(t)}{N(t)} \geq y(t)=\frac{I(0)}{N(0)} e^{-(\lambda+\gamma) t} \text { for } t \geq 0
$$

But then

$$
\frac{I\left(t^{*}-\Delta\right)}{N\left(t^{*}-\Delta\right)} \geq \frac{I(0)}{N(0)} e^{-(\lambda+\gamma)\left(t^{*}-\Delta\right)}
$$

assuming of course that $t^{*} \geq \Delta$, which we may henceforth assume without fear of contradiction. Inequality (8.19) will therefore be true if:

$$
\frac{I(0)}{N(0)} e^{-(\lambda+\gamma)\left(t^{*}-\Delta\right)} e^{(A-(\lambda+\gamma)) \Delta}>1
$$

This is equivalent to asking for:

$$
k_{3} e^{A \Delta-k_{4} t^{*}}>1
$$

where $k_{3}$ and $k_{4}$ are positive constants. (Specifically $k_{3}=\frac{I(0)}{N(0)}<1$ and $k_{4}=\lambda+\gamma$.) If we let

$$
A=\frac{k_{4} t^{*}}{\Delta}+\frac{1}{\Delta} \ln \left(\frac{1}{k_{3}}\right)+1
$$

it is clear that $(8.24)$ is satisfied. (Notice that $\ln \left(\frac{1}{k_{3}}\right)>0$ since $0<k_{3}<1$.) But now, in light of (8.25), condition (8.20) becomes:

$$
k_{1} e^{k_{2} t^{*}}>\frac{k_{4} t^{*}}{\Delta}+\frac{1}{\Delta} \ln \left(\frac{1}{k_{3}}\right)+1 .
$$

By standard facts concerning exponentials and linear functions, it is obvious that (8.26) will hold for all $t^{*}$ sufficiently large. Given such a $t^{*}$, we immediately have an appropriate choice for $A$ by (8.25). Then both (8.15) and (8.19) are satisfied for our choices of $t^{*}$ and $A$.

Given an $A$ and $t^{*}$ satisfying (8.19), we can write, by (8.17) and (8.18):

$$
\frac{I\left(t^{*}\right)}{N\left(t^{*}\right)}>1
$$

which contradicts positivity.

Thus our original assumption that $\frac{S(t)}{N(t)}$ does not tend to zero as $t \rightarrow \infty$ must be false, and so $\frac{S(t)}{N(t)} \rightarrow 0$ as $t \rightarrow \infty$.

As for the PVS models, we may use similar reasoning as that used for the CVS models with a few extra details. As we did with the CVS models, assume for the PVS models that there exists an $\epsilon>0$ such that there is no time after which $\frac{S(t)}{N(t)}$ is always less than $\epsilon$. So there will be a time $t^{*}$, arbitrarily large, such that $\frac{S\left(t^{*}\right)}{N\left(t^{*}\right)}>\epsilon$. But for any $t^{*} \geq t_{1}$, there exists $i \in \mathbb{N}$ such that $t^{*} \in\left[t_{i}, t_{i+1}\right)$.

Define $\epsilon_{1}=\min (\epsilon, 2 \lambda T)$. Then $0<\epsilon_{1} \leq \epsilon$. Also $\frac{\epsilon_{1}}{2 \lambda} \leq T$ and $\frac{S\left(t^{*}\right)}{N\left(t^{*}\right)}>\epsilon \geq \epsilon_{1}$. There are two cases to consider: 
(1) $t^{*}-t_{i} \geq \frac{\epsilon_{1}}{2 \lambda}$

(2) $t^{*}-t_{i}<\frac{\epsilon_{1}}{2 \lambda}$

Case (1). Define $\Delta=\frac{\epsilon_{1}}{2 \lambda}$. For this $\Delta$, we can argue on the interval $t \in\left[t^{*}-\Delta, t^{*}\right]$ (by techniques used for the CVS models, with $\epsilon$ replaced by $\epsilon_{1}$ ) that for $t^{*}$ sufficiently large, then $\frac{I\left(t^{*}\right)}{N\left(t^{*}\right)}>1$. The interval $t \in\left[t^{*}-\Delta, t^{*}\right]$ does not contain any pulses since by assumption $t_{i} \leq t^{*}-\Delta$ and we know that $t^{*}<t_{i+1}$. The argument will give us a lower bound $L_{1}$ for $t^{*}$ such that if case (1) holds, then a contradiction is obtained.

Case (2). The assumption in case (2) that $t^{*}-t_{i}<\frac{\epsilon_{1}}{2 \lambda}$ leads to the conclusion that $\frac{S\left(t_{i}\right)}{N\left(t_{i}\right)}>\frac{\epsilon_{1}}{2}$. To see this, suppose for a contradiction that $\frac{S\left(t_{i}\right)}{N\left(t_{i}\right)} \leq \frac{\epsilon_{1}}{2}$. As with the CVS models, we have, between pulses, that $\frac{d}{d t}\left(\frac{S(t)}{N(t)}\right)<\lambda$. Then $\frac{S(t)}{N(t)} \leq g(t)$ for $t \in\left[t_{i}, t^{*}\right]$ where $g\left(t_{i}\right)=\frac{\epsilon_{1}}{2} \geq \frac{S\left(t_{i}\right)}{N\left(t_{i}\right)}$ and $\frac{d g(t)}{d t}=\lambda$. Solving for $g(t)$ gives

$$
\frac{S(t)}{N(t)} \leq g(t)=\lambda\left(t-t_{i}\right)+\frac{\epsilon_{1}}{2} \text { for } t \in\left[t_{i}, t^{*}\right] .
$$

Hence $\frac{S\left(t^{*}\right)}{N\left(t^{*}\right)} \leq \lambda\left(t^{*}-t_{i}\right)+\frac{\epsilon_{1}}{2}<\epsilon_{1}$ by the assumption of case (2). But this is a contradiction since $\epsilon_{1} \leq \epsilon$.

Now since $\frac{S\left(t_{i}\right)}{N\left(t_{i}\right)}>\frac{\epsilon_{1}}{2}$ and $\frac{S\left(t_{i}\right)}{N\left(t_{i}\right)}=q \frac{S\left(t_{i}^{-}\right)}{N\left(t_{i}^{-}\right)}$(where $q=1-p$ ), we must have $\frac{S\left(t_{i}^{-}\right)}{N\left(t_{i}^{-}\right)}>\frac{\epsilon_{1}}{2 q}$. Let $\epsilon_{2}=\frac{\epsilon_{1}}{2 q}$. Define $\Delta=\min \left(\frac{\epsilon_{2}}{2 \lambda}, \frac{T}{2}\right)$. For this $\Delta$, we can argue on the interval $t \in\left[t_{i}-\Delta, t_{i}\right)$ (by techniques used for the CVS models, with $\epsilon$ replaced by $\left.\epsilon_{2}\right)$ that for $t_{i}$ sufficiently large, then $\frac{I\left(t_{i}^{-}\right)}{N\left(t_{i}^{-}\right)}>1$. The interval $\left.t \in\left[t_{i}-\Delta, t_{i}\right)\right]$ does not contain any pulses since here $\Delta<T$. The argument will give us a lower bound $L_{2}$ for $t_{i}$ (and hence a lower bound for $t^{*}$ ) such that if case (2) holds, then a contradiction is obtained.

For any $t^{*}$ larger than $\max \left(t_{1}, L_{1}, L_{2}\right)$, either case (1) or case (2) must hold. But then a contradiction must result. Hence $\frac{S(t)}{N(t)}$ must tend to zero as $t \rightarrow \infty$.

Lemma 8.1 gives us an insight into the long-term behaviour of the models, and enables us to determine other long-term behaviour, in terms of vaccination strategies, in the theorems and corollary in section 8.4 below. This is significant because public health authorities should be able to make long-term plans. On the other hand, such plans cannot be made without an understanding of just how long it takes for longterm behaviour to occur. In the proof of lemma 8.1, we find, for any $\epsilon>0$, that if $\frac{S\left(t^{*}\right)}{N\left(t^{*}\right)}>\epsilon$, then a contradiction is obtained if $t^{*}$ satisfies (8.26). But (8.26) is satisfied as soon as an exponential (with positive exponent) outgrows a linear function, which can be considered to mean "quickly". In this sense, behaviour predicted as long-term will be approached quickly. This is encouraging because the long-term validity of the models is open to debate - exponential population growth cannot occur indefinitely.

Knowing that the proportion of susceptibles tends to zero as time tends to infinity is more meaningful if we know how the number of susceptibles changes with time. The two lemmas which follow reveal the long-term behaviour of the susceptible population in all of our models. 
Lemma 8.2. In all the models (no VS, CVS, or PVS), when the incidence function is simple mass action, the number of susceptibles $S(t)$ is finite for $t>0$.

Proof. We consider the CVS model first. Now $\beta(N)=\eta N$ when the incidence is simple mass action. Also, we know by positivity that $\frac{I(t)}{N(t)} \leq 1$ for $t>0$ and that $-\mu S(t) \leq 0$ for $t>0$. Then, by (8.4), we can write, for $t>0$,

$$
\frac{d S(t)}{d t} \leq(1-\pi) \lambda N(t)-\eta N(t) S(t) .
$$

But $N(t)=N(0) e^{(\lambda-\mu) t}$ by (8.9). Hence, for $t>0$,

$$
\frac{d S(t)}{d t} \leq(1-\pi) \lambda N(0) e^{(\lambda-\mu) t}-\eta N(0) e^{(\lambda-\mu) t} S(t) .
$$

It follows that $S(t) \leq S_{1}(t)$ for $t>0$ where $S_{1}(0)=S(0)>0$ and where, for $t>0$,

$$
\frac{d S_{1}(t)}{d t}=(1-\pi) \lambda N(0) e^{(\lambda-\mu) t}-\eta N(0) e^{(\lambda-\mu) t} S_{1}(t)
$$

This is a linear first order ordinary differential equation, which we can trivially solve to obtain, for $t>0$,

$$
S_{1}(t)=(1-\pi)\left(\frac{\lambda}{\eta}\right)+\left[S(0)-(1-\pi)\left(\frac{\lambda}{\eta}\right)\right] e^{c} e^{-c e^{(\lambda-\mu) t}},
$$

where $c=\frac{\eta N(0)}{\lambda-\mu}$ is a positive constant. Clearly, then, $S_{1}(t)$ changes monotonically from $S(0)$ to $(1-\pi)\left(\frac{\lambda}{\eta}\right)$ as $t$ increases. Hence by (8.32) we have, for $t>0$,

$$
S(t) \leq S_{1}(t) \leq \max \left\{S(0),(1-\pi)\left(\frac{\lambda}{\eta}\right)\right\}
$$

Certainly, then, $S(t)$ is finite for $t>0$.

The no VS model is a special case of the CVS model. By setting $\pi=0$ in the above argument, we find that $S(t)$ is finite for $t>0$ in the no VS model.

As for the PVS model, we find, by reasoning along lines similar to those given in the first part of this proof up to (8.30), that

$$
\frac{d S(t)}{d t} \leq(1-\pi) \lambda N(0) e^{(\lambda-\mu) t}-\eta N(0) e^{(\lambda-\mu) t} S(t)
$$

for $t \in\left(0, t_{1}\right)$ and for $t \in\left(t_{i}, t_{i+1}\right)$ where $i \geq 1$. We also know by (8.7) that $S\left(t_{i}\right)=$ $q S\left(t_{i}^{-}\right)$for $i \geq 1$, where $q=1-p$ is a constant between zero and one, and where $t_{i}^{-}$ is the time "momentarily" before time $t_{i}$. Hence $S(t) \leq S_{2}(t)$ where $S_{2}(0)=S(0)$, where $S_{2}\left(t_{i}\right)=S_{2}\left(t_{i}^{-}\right)$for $i \geq 1$, and where

$$
\frac{d S_{2}(t)}{d t}=(1-\pi) \lambda N(0) e^{(\lambda-\mu) t}-\eta N(0) e^{(\lambda-\mu) t} S_{2}(t)
$$

for $t \in\left(0, t_{1}\right)$ and for $t \in\left(t_{i}, t_{i+1}\right)$ for $i \geq 1$. It follows that $S_{2}(t)$ satisfies (8.35) for $t>0$, so we can solve for $S_{2}(t)$ explicitly, bearing in mind that we have the initial condition $S_{2}(0)=S(0)$. By an argument very similar to that used above, from (8.31) to (8.33), we can therefore deduce that $S_{2}(t)$ is finite for $t>0$, so that $S(t)$ is also finite since $S(t) \leq S_{2}(t)$ for $t>0$. 
Since we know by (8.9) that the total population tends to infinity as time tends to infinity, it is trivially deduced by lemma 8.2 that the proportion of susceptibles tends to zero as time tends to infinity when the incidence is simple mass action. Thus, for the case of simple mass action incidence, we have uncovered a simpler proof that the proportion of susceptibles tends to zero as time tends to infinity than that provided by the proof of lemma 8.1. However, the value of the proof of lemma 8.1 is not diminished, for it also establishes, for the case of intermediate mass action incidence, that the proportion of susceptibles tends to zero as time tends to infinity, a result which is not trivial in view of the following lemma:

Lemma 8.3. In all the models (no VS, CVS, or PVS), when the incidence function is intermediate mass action, the number of susceptibles $S(t) \rightarrow \infty$ as $t \rightarrow \infty$.

Proof. We deal with the CVS model first. We will have $S(t) \rightarrow \infty$ as $t \rightarrow \infty$ if, for any $M>0$, there exists $t^{*}>0$ such that, for all $t \geq t^{*}$, we have $S(t) \geq M$. So, choose any $M>0$.

Now by positivity we know that $\frac{I(t)}{N(t)} \leq 1$ for $t>0$. Then, by the positivity of $S(t)$, we have $-\frac{S(t) I(t)}{N(t)} \geq-S(t)$ for $t>0$. Also, $\beta(N)=\Omega N^{v}$ when the incidence is intermediate mass action. Therefore, by (8.4), we can write, for $t>0$,

$$
\begin{aligned}
\frac{d S(t)}{d t} & \geq(1-\pi) \lambda N(t)-\left[\Omega(N(t))^{v}+\mu\right] S(t) \\
& =(1-\pi) \lambda N(t)\left(1-\left[\frac{\Omega(N(t))^{v}+\mu}{(1-\pi) \lambda N(t)}\right] S(t)\right)
\end{aligned}
$$

Since $0<v<1$ by assumption, we deduce by (8.9) that $\frac{\Omega(N(t))^{v}+\mu}{(1-\pi) \lambda N(t)} \rightarrow 0$ as $t \rightarrow \infty$. In particular, then, $\frac{\Omega(N(t))^{v}+\mu}{(1-\pi) \lambda N(t)}<\frac{1}{2 M}$ for all $t$ large enough (for all $t \geq \hat{t}$, say). Thus by (8.36) and (8.9) we find, for $t \geq \hat{t}$,

$$
\frac{d S(t)}{d t} \geq(1-\pi) \lambda N(0) e^{(\lambda-\mu) t}\left(1-\frac{1}{2 M} S(t)\right)
$$

It follows that $S(t) \geq S_{1}(t)$ for $t \geq \hat{t}$ where $S_{1}(\hat{t})=S(\hat{t})>0$ (recall that $S(t)>0$ for $t>0$ by strict positivity) and where, for $t>\hat{t}$,

$$
\frac{d S_{1}(t)}{d t}=(1-\pi) \lambda N(0) e^{(\lambda-\mu) t}\left(1-\frac{1}{2 M} S_{1}(t)\right) .
$$

This is a linear first order ordinary differential equation, which we can trivially solve to obtain, for $t \geq \hat{t}$,

$$
S(t) \geq S_{1}(t)=2 M+(S(\hat{t})-2 M) e^{-k\left(e^{(\lambda-\mu) t}-e^{(\lambda-\mu) \hat{t}}\right)},
$$

where $k=\frac{(1-\pi) \lambda N(0)}{2 M(\lambda-\mu)}$ is a positive constant. Clearly $e^{-k\left(e^{(\lambda-\mu) t}-e^{(\lambda-\mu) \hat{t}}\right)} \rightarrow 0$ as $t \rightarrow$ $\infty$. Hence there exists $t^{*} \geq \hat{t}$ such that, for all $t \geq t^{*}$, we can write

$$
(S(\hat{t})-2 M) e^{-k\left(e^{(\lambda-\mu) t}-e^{(\lambda-\mu) \hat{t}}\right)}>-M .
$$

Then, for $t \geq t^{*}$, we know by (8.39) that $S(t) \geq S_{1}(t)>M$. Since we assumed that $M$ can be any positive constant, we may deduce that $S(t) \rightarrow \infty$ as $t \rightarrow \infty$. 
Next notice that by setting $\pi=0$ in the above argument for the CVS model, we find that $S(t) \rightarrow \infty$ as $t \rightarrow \infty$ in the no VS model.

As for the PVS model, we know that, between pulses, equation (8.1) holds. By positivity we may deduce (as for the CVS model above) that $-\frac{S(t) I(t)}{N(t)} \geq-S(t)$. We also know that $\beta(N)=\Omega N^{v}$ because the incidence is intermediate mass action. Then we find that

$$
\frac{d S(t)}{d t} \geq \lambda N(t)\left(1-\left[\frac{\Omega(N(t))^{v}+\mu}{\lambda N(t)}\right] S(t)\right) \text { for } t \in\left(t_{i}, t_{i+1}\right), \quad i \geq 1,
$$

where (recall) $t_{i}$ is the time of the $i$-th pulse.

Now choose any $M>0$. In the PVS model, equation (8.9) holds for $t>0$. Also, $0<v<1$ by assumption. Recall that $p$ is the strength of each pulse and that $q=1-p$. By assumption $p<1$, so that $q>0$. Hence we know that $\frac{\Omega(N(t))^{v}+\mu}{\lambda N(t)}<\frac{q}{2 M}$ for all $t$ large enough (for all $t \geq \bar{t}$, say). Since $t_{i}=t_{1}+(i-1) T$ for $i \geq 2$ where $T$ is a positive constant, then there trivially exists $i^{*} \geq 1$ such that $t_{i}>\bar{t}$ for all $i \geq i^{*}$. Furthermore, by (8.7), we have $S\left(t_{i}\right)=q S\left(t_{i}^{-}\right)$for $i \geq 1$ where $t_{i}^{-}$is the time "momentarily" before time $t_{i}$. Combining the observations of this paragraph with (8.41), we find, for $i \geq i^{*}$, that

$$
\begin{aligned}
\frac{d S(t)}{d t} & \geq \lambda N(0) e^{(\lambda-\mu) t}\left(1-\frac{q}{2 M} S(t)\right) \quad \text { for } t \in\left(t_{i}, t_{i+1}\right) \\
S\left(t_{i}\right) & =q S\left(t_{i}^{-}\right) .
\end{aligned}
$$

Hence $S(t) \geq S_{2}(t)$ for $t \geq t_{i^{*}}$ where $S_{2}\left(t_{i^{*}}\right)=S\left(t_{i^{*}}\right)>0$ and where

$$
\begin{aligned}
\frac{d S_{2}(t)}{d t} & =\lambda N(0) e^{(\lambda-\mu) t}\left(1-\frac{q}{2 M} S_{2}(t)\right) \quad \text { for } t \in\left(t_{i}, t_{i+1}\right), \quad i \geq i^{*} \\
S_{2}\left(t_{i}\right) & =q S_{2}\left(t_{i}^{-}\right), \quad i \geq i^{*}+1 .
\end{aligned}
$$

For any $i \geq i^{*}$, we can solve (8.44) explicitly to find:

$$
S_{2}(t)=\frac{2 M}{q}+\left(S_{2}\left(t_{i}\right)-\frac{2 M}{q}\right) e^{-z\left(e^{(\lambda-\mu) t}-e^{(\lambda-\mu) t_{i}}\right)} \quad \text { for } t \in\left[t_{i}, t_{i+1}\right),
$$

where $z=\frac{\lambda N(0)}{2 M(\lambda-\mu)}$ is a positive constant. Clearly $S_{2}(t)$ in (8.46) is a monotonic function for $t \geq t_{i}$, beginning with value $S_{2}\left(t_{i}\right)$ and then moving monotonically towards $\frac{2 M}{q}$. Hence:

$$
S_{2}(t) \geq \min \left\{S_{2}\left(t_{i}\right), \frac{2 M}{q}\right\} \quad \text { for } t \in\left[t_{i}, t_{i+1}\right), \quad i \geq i^{*} .
$$

Given that $S_{2}\left(t_{i^{*}}\right)=S\left(t_{i^{*}}\right)$ and that $S\left(t_{i^{*}}\right)>0$ by the strict positivity of $S(t)$, and also given (8.45) and (8.47), we deduce that $S_{2}(t)>0$ for $t \geq t_{i^{*}}$.

By (8.45) and (8.46), we can write, for any $i \geq i^{*}$,

$$
\begin{aligned}
S_{2}\left(t_{i+1}\right) & =q S_{2}\left(t_{i+1}^{-}\right)=2 M+\left(q S_{2}\left(t_{i}\right)-2 M\right) e^{-z\left(e^{(\lambda-\mu) t_{i+1}}-e^{(\lambda-\mu) t_{i}}\right)} \\
& \geq 2 M\left(1-e^{-z\left(e^{(\lambda-\mu) t_{i+1}}-e^{(\lambda-\mu) t_{i}}\right)}\right) .
\end{aligned}
$$


Now $e^{(\lambda-\mu) t_{i+1}}-e^{(\lambda-\mu) t_{i}}=e^{(\lambda-\mu) t_{i}}\left(e^{(\lambda-\mu) T}-1\right)$ since $t_{i+1}-t_{i}=T$. But $e^{(\lambda-\mu) t_{i}} \rightarrow$ $\infty$ as $i \rightarrow \infty$ since $t_{i} \rightarrow \infty$ as $i \rightarrow \infty$. Also $e^{(\lambda-\mu) T}-1>0$. Hence:

$$
e^{(\lambda-\mu) t_{i}}\left(e^{(\lambda-\mu) T}-1\right) \rightarrow \infty \text { as } i \rightarrow \infty .
$$

But then, since $z$ is a positive constant, $e^{-z\left(e^{(\lambda-\mu) t_{i+1}}-e^{(\lambda-\mu) t_{i}}\right)} \rightarrow 0$ as $i \rightarrow \infty$. In particular, there exists $i^{* *} \geq 1$ such that $e^{-z\left(e^{(\lambda-\mu) t_{i+1}}-e^{(\lambda-\mu) t_{i}}\right)}<\frac{1}{2}$ for $i \geq i^{* *}$. Hence, for $i \geq \max \left\{i^{*}, i^{* *}\right\}$, we know by (8.48) that $S_{2}\left(t_{i+1}\right) \geq M$. Then, if we let $i^{* * *}=$ $\max \left\{i^{*}, i^{* *}\right\}+1$, we find by (8.47) that, for $i \geq i^{* * *}$,

$$
S_{2}(t) \geq \min \left\{M, \frac{2 M}{q}\right\} \quad \text { for } t \in\left[t_{i}, t_{i+1}\right) .
$$

But $0<q \leq 1$, so $\frac{2 M}{q}>M$. Also $S(t) \geq S_{2}(t)$ for $t \geq t_{i^{* * *}}$. Therefore $S(t) \geq$ $S_{2}(t) \geq M$ for $t \geq t_{i^{* * *}}$. For any $M>0$, then, there exists $t_{i^{* * *}}>0$ such that, for $t \geq t_{i^{* * *}}$, we have $S(t) \geq M$. In other words, $S(t) \rightarrow \infty$ as $t \rightarrow \infty$.

\subsection{Vaccination strategies}

In this section we discover the long-term effect on the proportion of infectives when there is no vaccination strategy (no VS), a constant vaccination strategy (CVS), and when there is a pulse vaccination strategy (PVS).

Theorem 8.1. When the incidence function is simple or intermediate mass action, then for the no VS and CVS models (when $\pi<1$ ), we have $\frac{I(t)}{N(t)} \rightarrow \frac{\lambda(1-\pi)}{\gamma+\lambda}$ as $t \rightarrow \infty$. Thus the constant vaccination strategy fails to eradicate the disease relatively, though it does reduce the ultimate infective proportion.

Proof. We prove the result for the CVS models. The no VS models are trivially dealt with by setting $\pi=0$ in what follows. Using equations (8.6) and (8.8), we can write:

$$
\frac{d}{d t}\left(\frac{R(t)}{N(t)}\right)=\gamma\left(\frac{I}{N}\right)+\lambda \pi-\lambda\left(\frac{R}{N}\right)
$$

Now we know by lemma 8.1 that, for any $\epsilon>0$, there exists $\bar{t}>0$ such that, for all $t>\bar{t}$, we have $\frac{S(t)}{N(t)}<\epsilon$. But then, since $\frac{S(t)}{N(t)}+\frac{I(t)}{N(t)}+\frac{R(t)}{N(t)}=1$, and since we have strict positivity, we must have, for $t>\bar{t}$,

$$
1-\frac{R(t)}{N(t)}-\epsilon \leq \frac{I(t)}{N(t)} \leq 1-\frac{R(t)}{N(t)}
$$

and hence, by (8.50) and (8.51), we have, for $t>\bar{t}$ :

$$
\gamma(1-\epsilon)+\lambda \pi-(\lambda+\gamma)\left(\frac{R}{N}\right) \leq \frac{d}{d t}\left(\frac{R(t)}{N(t)}\right) \leq \gamma+\lambda \pi-(\lambda+\gamma)\left(\frac{R}{N}\right) .
$$
for $t>\bar{t}$ :

Therefore, for $t \geq \bar{t}$, we have $x_{1}(t) \leq \frac{R(t)}{N(t)} \leq x_{2}(t)$ where $x_{1}(\bar{t})=\frac{R(\bar{t})}{N(\bar{t})}=x_{2}(\bar{t})$ and,

$$
\frac{d x_{1}(t)}{d t}=\gamma(1-\epsilon)+\lambda \pi-(\lambda+\gamma) x_{1}(t)
$$


and

$$
\frac{d x_{2}(t)}{d t}=\gamma+\lambda \pi-(\lambda+\gamma) x_{2}(t) .
$$

The differential equations for $x_{1}(t)$ and $x_{2}(t)$ are linear and first order, so either by direct calculation or by a standard phase portrait analysis we may quickly deduce, that, for $t$ big enough, $x_{1}(t)$ is arbitrarily close to $\frac{\gamma(1-\epsilon)+\lambda \pi}{\gamma+\lambda}$ and $x_{2}(t)$ is arbitrarily close to $\frac{\gamma+\lambda \pi}{\gamma+\lambda}$. Then, for any $\epsilon_{1}>0$, there exists $t^{*} \geq \bar{t}$ such that, for $t \geq t^{*}$, we have

$$
\left|x_{1}(t)-\left(\frac{\gamma(1-\epsilon)+\lambda \pi}{\gamma+\lambda}\right)\right|<\epsilon_{1} \quad \text { and } \quad\left|x_{2}(t)-\left(\frac{\gamma+\lambda \pi}{\gamma+\lambda}\right)\right|<\epsilon_{1} .
$$

But then, for $t \geq t^{*}$,

$$
\left(\frac{\gamma+\lambda \pi}{\gamma+\lambda}\right)-\left(\frac{\epsilon \gamma}{\lambda+\gamma}+\epsilon_{1}\right)<x_{1}(t) \leq \frac{R(t)}{N(t)} \leq x_{2}(t)<\left(\frac{\gamma+\lambda \pi}{\gamma+\lambda}\right)+\epsilon_{1} .
$$

Now (8.56) is true for any $\epsilon>0$ and any $\epsilon_{1}>0$, so if we set $\epsilon_{2}=\frac{\epsilon \gamma}{\lambda+\gamma}+\epsilon_{1}$, it follows that for any $\epsilon_{2}>0$, there exists $t^{*}>0$ such that, for $t \geq t^{*}$, then

$$
\left|\frac{R(t)}{N(t)}-\left(\frac{\gamma+\lambda \pi}{\gamma+\lambda}\right)\right|<\epsilon_{2} .
$$

In other words, $\frac{R(t)}{N(t)} \rightarrow \frac{\gamma+\lambda \pi}{\gamma+\lambda}$ as $t \rightarrow \infty$. Since also $\frac{S(t)}{N(t)} \rightarrow 0$ as $t \rightarrow \infty$ by lemma 8.1, then:

$$
\frac{I(t)}{N(t)}=1-\frac{S(t)}{N(t)}-\frac{R(t)}{N(t)} \rightarrow 1-\frac{\gamma+\lambda \pi}{\gamma+\lambda}=\frac{\lambda(1-\pi)}{\gamma+\lambda} \text { as } t \rightarrow \infty .
$$

Theorem 8.2. When the incidence function is simple or intermediate mass action, then for the PVS model (when $p<1$ ), we have $\frac{I(t)}{N(t)} \rightarrow \frac{\lambda}{\lambda+\gamma}$ as $t \rightarrow \infty$. Thus the pulse vaccination strategy fails to eradicate the disease relatively.

Proof. Choose any $\epsilon>0$. By lemma 8.1 we know that $\frac{S(t)}{N(t)}<\epsilon$ for all $t$ large enough. But then, since $t_{i}=t_{1}+(i-1) T \rightarrow \infty$ as $i \rightarrow \infty$, we know that there exists $i^{*}>0$ such that $\frac{S(t)}{N(t)}<\epsilon$ for $t \in\left[t_{i}, t_{i+1}\right)$ for all $i \geq i^{*}$. Now the PVS models evolve between pulses according to the same equations as in the non-vaccination models, so we find by the same reasoning (with $\pi=0$ ) as at the start of theorem 8.1 that, for $t \in\left(t_{i}, t_{i+1}\right)$ for $i \geq i^{*}$,

$$
\gamma(1-\epsilon)-(\lambda+\gamma)\left(\frac{R}{N}\right) \leq \frac{d}{d t}\left(\frac{R(t)}{N(t)}\right) \leq \gamma-(\lambda+\gamma)\left(\frac{R}{N}\right)
$$

Therefore,

$$
x_{1}(t) \leq \frac{R(t)}{N(t)} \leq x_{2}(t) \text { for } t \in\left[t_{i^{*}}, t_{i^{*}+1}\right),
$$

where $x_{1}\left(t_{i^{*}}\right)=\frac{R\left(t_{i^{*}}\right)}{N\left(t_{i^{*}}\right)}=x_{2}\left(t_{i^{*}}\right)$ and where, for $t \in\left(t_{i^{*}}, t_{i^{*}+1}\right)$ :

$$
\frac{d x_{1}(t)}{d t}=\gamma(1-\epsilon)-(\lambda+\gamma) x_{1}(t)
$$


and

$$
\frac{d x_{2}(t)}{d t}=\gamma-(\lambda+\gamma) x_{2}(t)
$$

Now by (8.60) we can write:

$$
x_{1}\left(t_{i^{*}+1}^{-}\right) \leq \frac{R\left(t_{i^{*}+1}^{-}\right)}{N\left(t_{i^{*}+1}^{-}\right)} \leq x_{2}\left(t_{i^{*}+1}^{-}\right) .
$$

But, since $R(t)$ is impulsively increased at the $i$-th pulse (see equation (8.7)), we know that:

$$
\frac{R\left(t_{i+1}^{-}\right)}{N\left(t_{i+1}^{-}\right)} \leq \frac{R\left(t_{i+1}\right)}{N\left(t_{i+1}\right)}<\frac{R\left(t_{i+1}^{-}\right)}{N\left(t_{i+1}^{-}\right)}+\epsilon \text { for } i \geq i^{*}
$$

Hence if we set $x_{1}\left(t_{i^{*}+1}\right)=x_{1}\left(t_{i^{*}+1}^{-}\right)$and $x_{2}\left(t_{i^{*}+1}\right)=x_{2}\left(t_{i^{*}+1}^{-}\right)+\epsilon$, we find by (8.63) and (8.64), that

$$
x_{1}\left(t_{i^{*}+1}\right) \leq \frac{R\left(t_{i^{*}+1}\right)}{N\left(t_{i^{*}+1}\right)} \leq x_{2}\left(t_{i^{*}+1}\right) .
$$

But then, using (8.65) and (8.59), we can say that $x_{1}(t) \leq \frac{R(t)}{N(t)} \leq x_{2}(t)$ for $t \in$ $\left[t_{i^{*}+1}, t_{i^{*}+2}\right)$, where $x_{1}\left(t_{i^{*}+1}\right)=x_{1}\left(t_{i^{*}+1}^{-}\right)$and $x_{2}\left(t_{i^{*}+1}\right)=x_{2}\left(t_{i^{*}+1}^{-}\right)+\epsilon$, and where, for $t \in\left(t_{i^{*}+1}, t_{i^{*}+2}\right), x_{1}(t)$ satisfies (8.61) and $x_{2}(t)$ satisfies (8.62).

Our argument for bounding $\frac{R(t)}{N(t)}$ for $t \in\left[t_{i^{*}+1}, t_{i^{*}+2}\right)$ can be repeated for $t \in$ $\left[t_{i^{*}+2}, t_{i^{*}+3}\right)$, for $t \in\left[t_{i^{*}+3}, t_{i^{*}+4}\right)$, and so on. Thus we have established that, for $i \geq i^{*}$, then $x_{1}(t) \leq \frac{R(t)}{N(t)} \leq x_{2}(t)$ for $t \in\left[t_{i}, t_{i+1}\right)$, where $x_{1}\left(t_{i^{*}}\right)=\frac{R\left(t_{i^{*}}\right)}{N\left(t_{i^{*}}\right)}=x_{2}\left(t_{i^{*}}\right)$, and

$$
x_{1}\left(t_{i}\right)=x_{1}\left(t_{i}^{-}\right) \text {and } x_{2}\left(t_{i}\right)=x_{2}\left(t_{i}^{-}\right)+\epsilon \text { for } i \geq i^{*}+1,
$$

and where, for $t \in\left(t_{i}, t_{i+1}\right)$ for $i \geq i^{*}, x_{1}(t)$ satisfies (8.61) and $x_{2}(t)$ satisfies (8.62).

Solving for $x_{2}(t)$ on $\left[t_{i}, t_{i+1}\right)$ for any $i \geq i^{*}$ gives:

$$
x_{2}(t)=\frac{\gamma}{\lambda+\gamma}+\left(x_{2}\left(t_{i}\right)-\frac{\gamma}{\lambda+\gamma}\right) e^{-(\lambda+\gamma)\left(t-t_{i}\right)}
$$

Hence:

$$
x_{2}\left(t_{i+1}^{-}\right)=\frac{\gamma}{\lambda+\gamma}+\left(x_{2}\left(t_{i}\right)-\frac{\gamma}{\lambda+\gamma}\right) e^{-(\lambda+\gamma) T} .
$$

Then, from (8.68), and using (8.66), we have:

$$
x_{2}\left(t_{i+1}\right)=\frac{\gamma}{\lambda+\gamma}+\epsilon+\left(x_{2}\left(t_{i}\right)-\frac{\gamma}{\lambda+\gamma}\right) e^{-(\lambda+\gamma) T} .
$$

Let $x_{2}\left(t_{i}\right)=z_{i}$. Then (8.69) gives, for $i \geq i^{*}$ :

$$
z_{i+1}=\frac{\gamma}{\lambda+\gamma}\left(1-e^{-(\lambda+\gamma) T}\right)+\epsilon+e^{-(\lambda+\gamma) T} z_{i} .
$$

This last equation is a one-dimensional map, which we can iterate to find an expression for $z_{i^{*}+j}$, for $j \geq 1$, in terms of $z_{i^{*}}$. The expression we obtain is readily 
verified by induction. Letting $A=\frac{\gamma}{\lambda+\gamma}\left(1-e^{-(\lambda+\gamma) T}\right)+\epsilon$ and $B=e^{-(\lambda+\gamma) T}$, we find that:

$$
z_{i^{*}+j}=\frac{A\left(1-B^{j}\right)}{1-B}+B^{j} z_{i^{*}}
$$

Since $0<B<1$, we see that $z_{i^{*}+j} \rightarrow \frac{A}{1-B}$ as $j \rightarrow \infty$. Hence we have:

$$
x_{2}\left(t_{i}\right)=z_{i} \rightarrow \frac{\gamma}{\lambda+\gamma}+\frac{\epsilon}{1-e^{-(\lambda+\gamma) T}} \text { as } i \rightarrow \infty .
$$

Thus, for any $\epsilon_{1}>0$, there exists $\bar{i} \geq i^{*}$ such that, for $i \geq \bar{i}$, then

$$
\left|x_{2}\left(t_{i}\right)-\left(\frac{\gamma}{\lambda+\gamma}+\frac{\epsilon}{1-e^{-(\lambda+\gamma) T}}\right)\right|<\epsilon_{1} .
$$

Let $\epsilon_{2}=\epsilon_{1}+\frac{\epsilon}{1-e^{-(\lambda+\gamma) T}}$. Since our arguments so far have applied for any $\epsilon>0$ and any $\epsilon_{1}>0$, we can conclude by (8.73) that, for any $\epsilon_{2}>0$, there exists $\bar{i}>0$ such that, for $i \geq \bar{i}$, then $\left|x_{2}\left(t_{i}\right)-\left(\frac{\gamma}{\lambda+\gamma}\right)\right|<\epsilon_{2}$. Hence $x_{2}\left(t_{i}\right) \rightarrow \frac{\gamma}{\lambda+\gamma}$ as $i \rightarrow \infty$.

By (8.67) we know that $x_{2}(t)$ is monotonic for $t \in\left[t_{i}, t_{i+1}\right)$ for $i \geq i^{*}$. But then an upper bound for $x_{2}(t)$ for $t \in\left[t_{i}, t_{i+1}\right)$ will be $\max \left\{x_{2}\left(t_{i}\right), x_{2}\left(t_{i+1}^{-}\right)\right\}$. But $x_{2}\left(t_{i}\right) \rightarrow \frac{\gamma}{\lambda+\gamma}$ as $i \rightarrow \infty$, so, using (8.66),

$$
x_{2}\left(t_{i+1}^{-}\right)=x_{2}\left(t_{i+1}\right)-\epsilon \leq x_{2}\left(t_{i+1}\right) \rightarrow \frac{\gamma}{\lambda+\gamma} \text { as } i \rightarrow \infty .
$$

So for any $\epsilon_{3}>0$ there exists $\hat{i}>0$ such that, for $i \geq \hat{i}$, we have

$$
x_{2}(t) \leq \frac{\gamma}{\lambda+\gamma}+\epsilon_{3} \text { for } t \in\left[t_{i}, t_{i+1}\right) .
$$

Similarly we can show that, for any $\epsilon_{4}>0$, there exists $\check{i}>0$ such that, for $i \geq \check{i}$, we have

$$
x_{1}(t) \geq \frac{\gamma}{\lambda+\gamma}-\epsilon_{4} \text { for } t \in\left[t_{i}, t_{i+1}\right) .
$$

Then, by (8.74) and (8.75), we know that for any $\epsilon_{5}>0$, there exists $\tilde{i}=\max \{\hat{i}, \check{i}\}$ such that, for $i \geq \tilde{i}$, we have

$$
\frac{\gamma}{\lambda+\gamma}-\epsilon_{5} \leq x_{1}(t) \leq \frac{R(t)}{N(t)} \leq x_{2}(t) \leq \frac{\gamma}{\lambda+\gamma}+\epsilon_{5} \text { for } t \in\left[t_{i}, t_{i+1}\right) .
$$

In other words, $\left|\frac{R(t)}{N(t)}-\left(\frac{\gamma}{\lambda+\gamma}\right)\right|<\epsilon_{5}$ for $t \geq t_{\tilde{i}}$. Certainly, then, $\frac{R(t)}{N(t)} \rightarrow \frac{\gamma}{\lambda+\gamma}$ as $t \rightarrow \infty$. Using this, and also using lemma 8.1, we can write:

$$
\frac{I(t)}{N(t)}=1-\frac{S(t)}{N(t)}-\frac{R(t)}{N(t)} \rightarrow 1-\frac{\gamma}{\gamma+\lambda}=\frac{\lambda}{\gamma+\lambda} \text { as } t \rightarrow \infty
$$

Corollary 8.1. When the incidence function is simple or intermediate mass action, then for all models (no VS, CVS (when $\pi<1$ ), or PVS (when $p<1$ )), we have $I(t) \rightarrow \infty$ as $t \rightarrow \infty$. Thus the vaccination strategies fail to eradicate the disease absolutely. 
Proof. By theorems 8.1 and 8.2 we see that, for all models, $\frac{I(t)}{N(t)}$ tends to a strictly positive constant as $t \rightarrow \infty$. But then, since $N(t) \rightarrow \infty$ by equation (8.9), we must have $I(t) \rightarrow \infty$ as $t \rightarrow \infty$.

From the results in this section, we can say that, when the incidence is either simple or intermediate mass action, both a CVS and a PVS fail dramatically to eradicate the disease absolutely, and whilst both also fail to eradicate the disease relatively, a CVS reduces the long-term proportion of infectives but a PVS does not. How can we make sense of the fact that a PVS does not reduce the long-term proportion of infectives when a CVS does reduce this? We can answer such a question by thinking about how the vaccination rate changes as time passes. When there is a CVS, the vaccination rate is $(1-\pi) \lambda N(t)$ by $(8.4)$, so the vaccination rate increases at the same rate at which the population grows. On the other hand, when there is a PVS, there are $p S(t)$ vaccinations every $T$ time units. But by lemma 8.1, $S(t)$ becomes negligible compared to $N(t)$ as time grows, so the size of the vaccination rate becomes negligible compared to the size of the population as time grows. In essence, then, the population outgrows the vaccination rate to such an extent that the PVS becomes ineffective.

In all of our models, it is curious that, even though the proportion of the population that can catch the disease tends to zero (by lemma 8.1), the proportion of the population having the disease tends to a positive constant. One might expect that if the stockpile of potential new cases dies out as a proportion of the population, then so might the disease. Instead the rapid growth of the population ensures a ready supply of new victims, and because the contact rate increases with the population, the disease spreads so quickly that susceptibles are infected even more quickly than the population grows.

\subsection{Simulations}

Figure 8.1 shows, for simple mass action incidence, simulations of the proportions of susceptibles, infectives, and removeds in the absence of vaccination, or when there is a CVS, or when there is a PVS. Figure 8.2 shows the same types of simulation but the incidence here is intermediate mass action. The simulation with simple mass action for $\frac{S(t)}{N(t)}$ when there is a PVS (figure 8.1, bottom left) is shown only from the commencement of the PVS, otherwise the detail is lost. Notice in this simulation that the impact of each pulse is almost instantaneously forgotten by the system. The pulses have a bigger impact, at least for the early pulses, when the incidence is intermediate mass action (figure 8.2, bottom row), which is likely to be because the contact rate is smaller, so that $\frac{S(t)}{N(t)}$ tends to zero more slowly.

Figure 8.3 (left) shows that, for simple mass action incidence, the number of infectives can decrease, over a limited time span, even in the absence of vaccinations, and despite the fact that the number of infectives is known (by the corollary) to tend to infinity. Such a simulation allows us to hope that the disease could die out naturally in certain real-world situations. In the real world, stochastic fluctuations become significant when numbers are low - a few fluctuations could reduce low infective numbers to zero. Figure 8.3 (right) shows that, alternatively, the number of infectives can increase from time $t=0$ and show no sign of ever decreasing. (It is incidentally trivial 

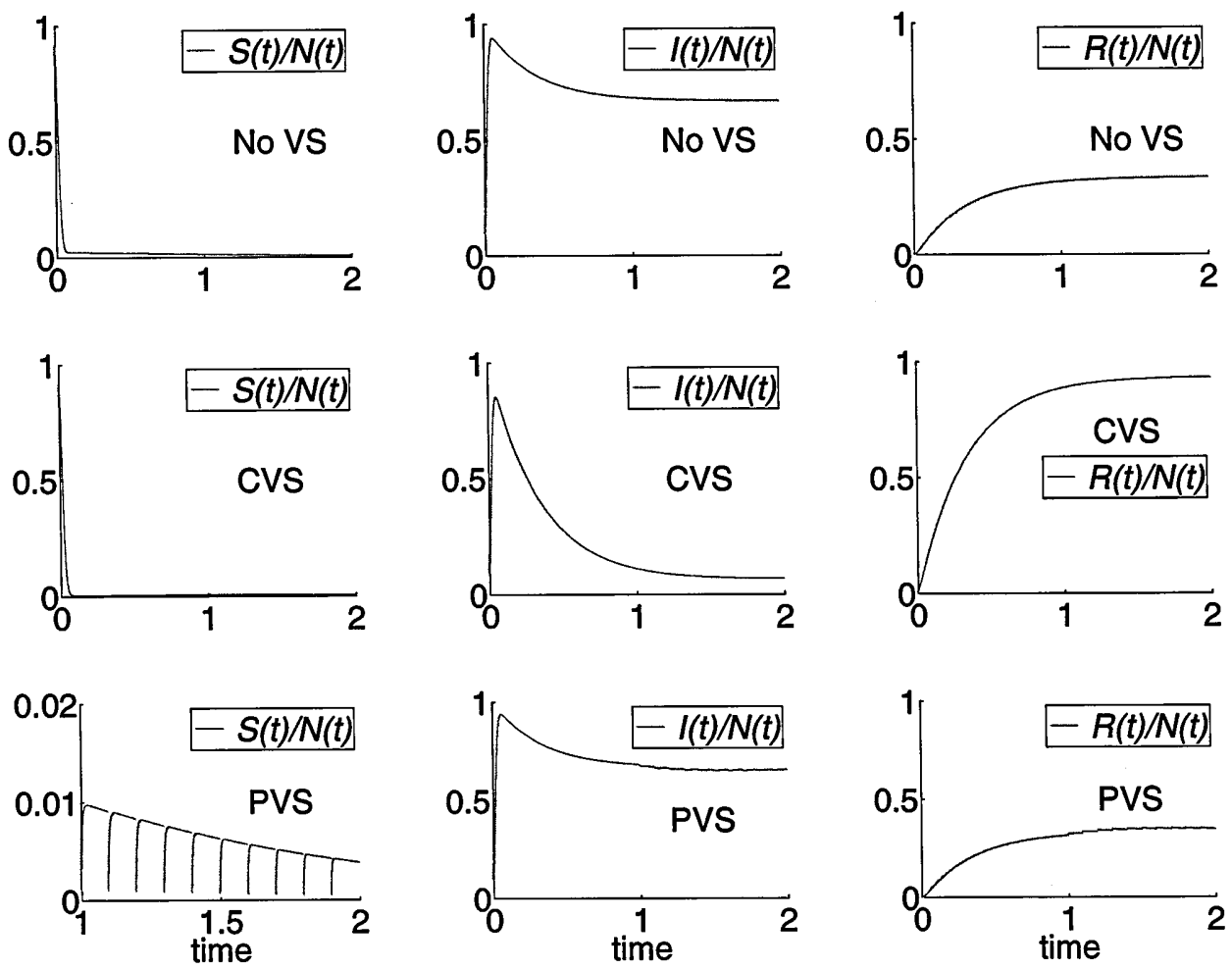

Figure 8.1: Simple mass action. Initial data: $(S(0), I(0), R(0))=(100,10,0)$. Model parameters: $\lambda=2, \mu=1, \gamma=1, \eta=1$. Constant vaccination strength: $\pi=0.9$. Pulse vaccination parameters: $t_{1}=1, p=0.9, T=0.1$. In the bottom left plot, the simulations is shown only from the commencement of the PVS, otherwise the detail is lost. 

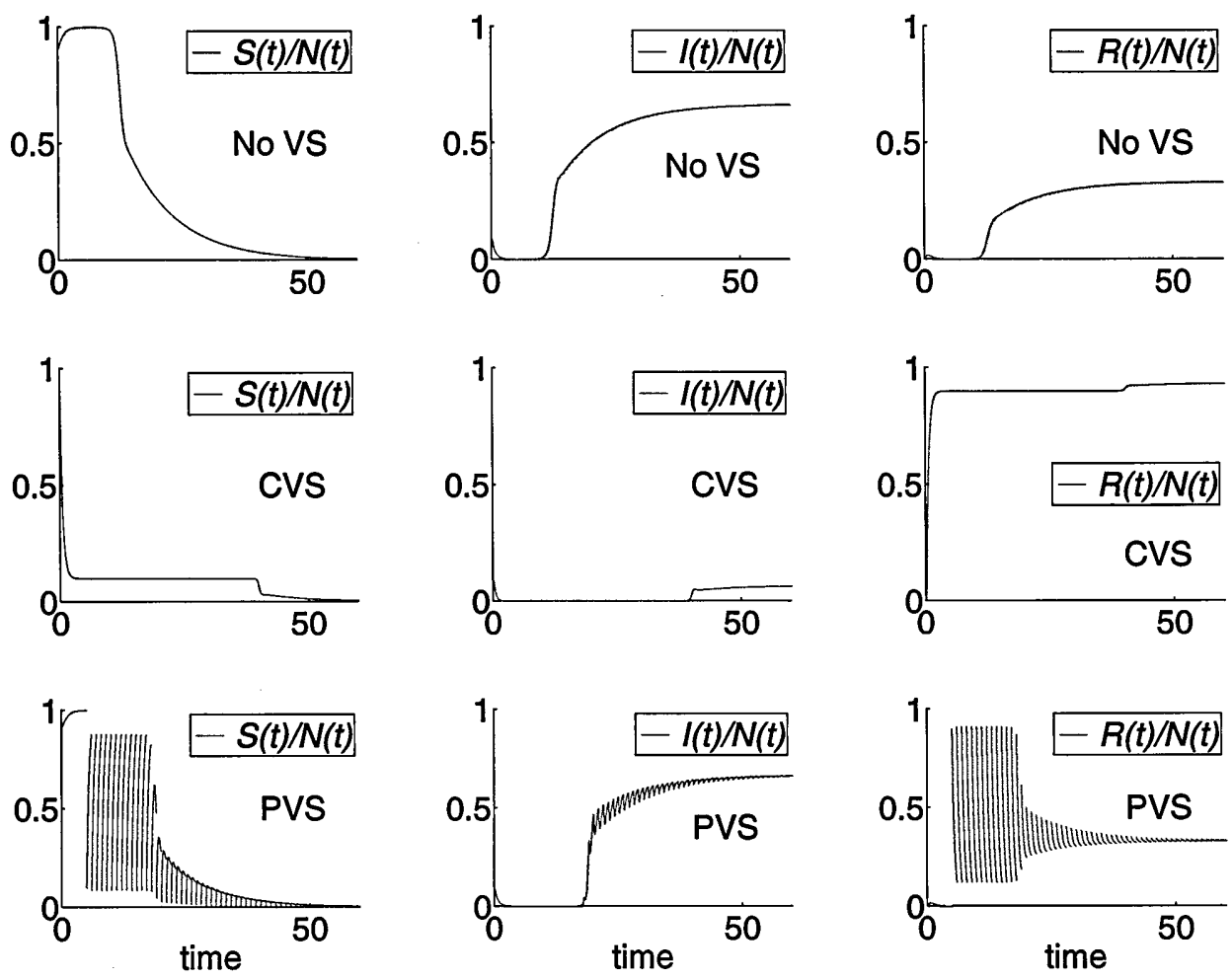

Figure 8.2: Intermediate mass action. Initial data: $(S(0), I(0), R(0))=(100,10,0)$. Model parameters: $\lambda=2, \mu=1, \gamma=1, \Omega=1, v=0.1$. Constant vaccination strength: $\pi=0.9$. Pulse vaccination parameters: $t_{1}=5, p=0.9, T=1$. 

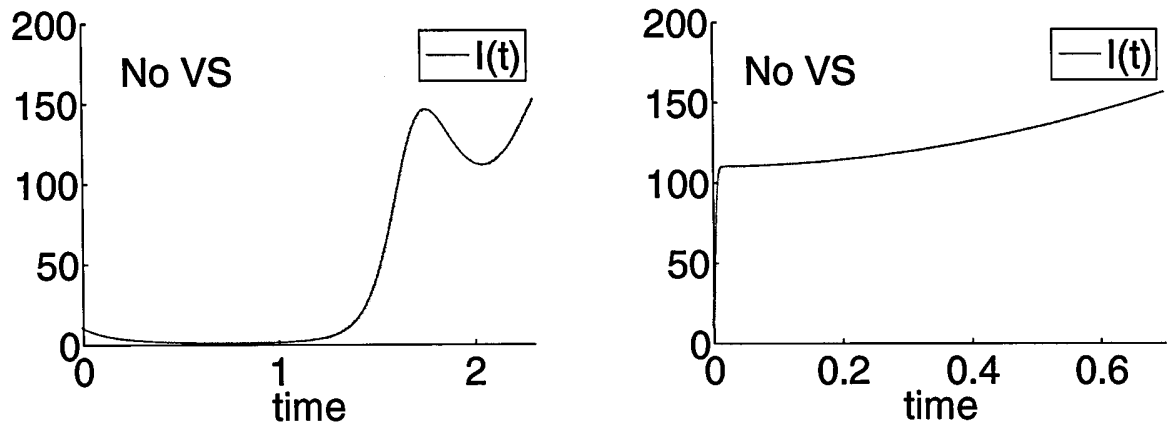

Figure 8.3: Simple mass action. Infective numbers. Initial data: $(S(0), I(0), R(0))=$ $(100,10,0)$. Model parameters: $\lambda=2, \mu=1$. Left: $\gamma=10, \eta=0.05$. Right: $\gamma=1$, $\eta=5$.
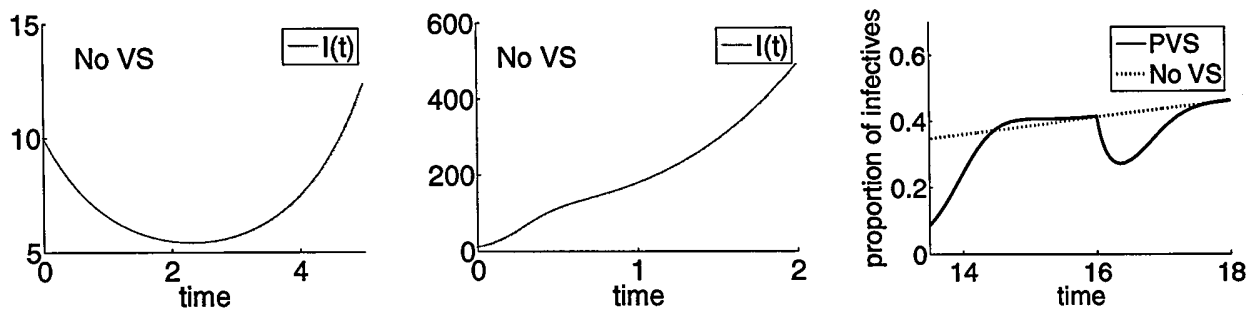

Figure 8.4: Intermediate mass action. Initial data: $(S(0), I(0), R(0))=(100,10,0)$. Model parameters: $\lambda=2, \mu=1, \gamma=1, \Omega=1$. Left and middle: Infective numbers. Left: $v=0.1$. Middle: $v=0.5$. Right: Infrequent pulses can increase infective proportion over a limited time range. Here $v=0.1, t_{1}=1, p=0.95, T=5$.

to deduce the sign of $\frac{d I(t)}{d t}$ at time $t=0$ by substituting the initial conditions into (8.2) and using the fact that $\beta(N)=\eta N$ when the incidence is simple mass action.)

Figure 8.4 (left and middle) reproduce the observations of the previous paragraph for intermediate mass action and figure 8.4 (right) shows that, for intermediate mass action, a PVS in which the inter-pulse time $T$ is not suitably small, may, over a limited time span, increase the proportion of infectives to a level higher than it would be in the absence of vaccination. This bolsters the conclusion by Choisy et al [61] that a PVS should be designed with care (see section 7.4 for additional comments on the paper by Choisy et al). We are not aware of any analytical demonstration of the potential harm of a poorly designed PVS.

\subsection{Discussion}

We have drawn attention to the tendency of human populations to grow in recent decades and centuries, leading to higher population densities which have been linked to higher contact rates. We have also noted that there are infectious diseases that have 
a low mortality rate (such as measles, varicella (chickenpox), mumps, and pertussis (whooping cough) [30]), the mortality rate being small enough to make plausible the idea of modelling such a disease as non-fatal. This all led us to consider an SIR model for a non-fatal infectious disease, circulating in an exponentially growing population in which the contact rate increases with the population. We have seen that if the contact rate increases linearly with the population size (the case of simple mass action incidence), and if there are strictly positive initial values for the numbers of susceptibles and infectives, and no vaccinations, then the number of infectives tends to infinity and the proportion of infectives tends to a strictly positive constant as time tends to infinity. These results still hold if there is either a constant vaccination strategy (CVS) with incomplete coverage or a pulse vaccination strategy (PVS) with incomplete coverage. Yet whilst a PVS does not change the positive constant to which the proportion of infectives tends, a CVS reduces it. All of these results again hold when the contact rate is proportional to a power $v$ of the population where $v$ is a constant with $0<v<1$ (the case of intermediate mass action).

Simulations have revealed, on the other hand, that, over a limited time span and for certain parameter choices and initial conditions, the number of infectives can decrease, for either simple or intermediate mass action, in the absence of vaccination, allowing us to believe that, under certain circumstances, the disease could die out naturally in the real-world by stochastic fluctuations. A simulation has also revealed (in the case of intermediate mass action) that a PVS may, over a limited time span, increase the proportion of infectives to a level higher than it would be in the absence of vaccination, strengthening the conclusion by Choisy et al [61] that a PVS should be designed with care. The simulations were included to demonstrate the range of qualitative behaviours possible in our models. Our theorems carry greater weight as a basis for decisions on matters of public health.

What are the implications of our theoretical results for public health control policies? Since it should never be assumed that vaccination coverage will always be complete - various factors can hinder vaccination programs (see section 7.6) - we are left with evidence that, for a non-fatal disease in an exponentially growing population, a CVS will reduce the proportion of infectives but a PVS will not. There are mathematical results establishing that, in a constant population, a PVS with sufficiently frequent pulses can bring a disease under control by lower coverage for each pulse than the coverage of newborns needed in a successful CVS [70,123]. We now know that such results do not necessarily carry over into models with a growing population.

Of course no population is likely to grow forever, but as long as rapid growth is occurring, the evidence in this chapter should not be ignored. Our models suggest that a CVS is a safer control option than a PVS for a non-fatal disease in a population experiencing steady long-term growth. The long-term prediction of population change should clearly then be an important aspect of public health policy. In order that we may contruct appropriate models, the influence of population and social changes on contact rates should be studied at the same time. Fortunately many investigators already seem to be aware of the importance of studying population change and the contact structure of populations [72, 90, 91, 113, 118, 126]. Our results help to confirm that such investigations are sensible.

Ideas for future research include allowing average contact behaviour to vary between individuals, or the incorporation of disease-caused deaths into our models, or the adjustment of the birth term to a logistic function preventing the indefinite growth of the population. An analytical demonstration of the potential harm of a poorly designed PVS would also have value. 


\section{Chapter 9}

\section{Pulse vaccination strategies in a metapopulation SIR model}

"Great things are not done by impulse, but by a series of small things brought together."

Vincent Van Gogh (1853 - 1890)

\subsection{Introduction}

Following the global eradication by 1979 of smallpox, the World Health Organisation has set as goals the global eradication of other diseases, including poliomyelitis [19] and dracunculiasis [29, 35], and the global reduction of, for example, measles [27]. Global eradication of a disease obviously requires eradication from every single region or country in the world. But if, for example, a vaccination program is to be implemented in any particular region, some level of participation or co-ordination will be required by existing health authorities in that region.

Suppose a disease is present in a number of different regions between any pair of which migration may occur. This is a common enough scenario in our world today - economic globalisation and the popularity of international holidays have promoted the development of transport links. Assume that each region has an autonomous public health authority that wishes to eradicate the disease from its region. If one authority were to implement a vaccination program in the region it controls, there would be no guarantee of eradication in that region since new infectives could enter it from the other regions. All of the regions agree, then, after common consultation, to implement vaccination programs. The regions share information on migration rates and on other parameters governing the spread of the disease such as birth rates, death rates, contact rates, and recovery rates. Each region decides to implement a pulse vaccination strategy (PVS), perhaps in consequence of a recommendation by the World Health Organisation that this is a sensible method of control for the disease in contention. (It is certainly true that pulse vaccination strategies have attained real-world success in the control of poliomyelitis and measles in Central and South America [123] 
and of poliomyelitis in India [85].) However, each autonomous public health authority wishes to retain some independence in choosing the exact details of the PVS in its region. After all, the days appropriate for a vaccination pulse in one region may not be appropriate in another - different countries have different customs, national holidays, and election days. The question therefore arises as to whether or not it is possible to eradicate the disease from all regions if the PVS in each region is chosen with some freedom. In this chapter we discover, for a particular model in which long-term exponential population growth does not occur (unlike in the models of chapter 8), that such eradication is possible, provided each PVS is sufficiently strong.

The model we will study will be for a disease with SIR-type dynamics on $n \geq 2$ regions or "patches", applicable to a disease such as poliomyelitis, influenza, measles, or rubella. Multi-patch disease models are often labelled as metapopulation models in the literature and the populations on the different patches are sometimes called subpopulations [49, 73, 101]. Pulse vaccination strategies in a two-patch SEIR model have been simulated by Earn et al [73]. Their simulation shows that the infective populations on the different patches can become synchronised (that is, they can become identical) by the influence of the pulse vaccination strategies. The pulse vaccination strategies on the two patches are identical in their simulation. Earn et al offer no analytical explanation for their observation but they do comment how Heino et al [86] have stressed that synchronicity between subpopulations in ecological models can be an important contributory factor to the extinction of all the subpopulations. Earn $e t$ al suggest that if pulse vaccination strategies can promote synchronicity on the different patches of a multi-patch epidemiological model, then disease extinction on all the patches may become more likely. We will not explore synchronicity in this thesis but remark that such an exploration could yield valuable new results.

This chapter has the following format. In section 9.2 we describe the $n$-patch SIR model to be investigated. In section 9.3 we consider the behaviour of the disease in the absence of vaccinations, showing in particular that it may die out naturally or persist indefinitely depending on conditions involving the model parameters. Then in sections 9.4 and 9.5 we construct conditions on pulse vaccination strategies on the $n$ patches such that the disease will be eradicated simultaneously on all $n$ patches. Simulations are included in section 9.6 and we end the chapter with a discussion in section 9.7 .

\subsection{The model}

Assume there are $n \geq 2$ patches on each of which a human population is present. Suppose an infectious disease with SIR-type dynamics is circulating on at least one of the patches. For $1 \leq j \leq n$, let there be $S_{j}(t)$ susceptibles, $I_{j}(t)$ infectives, and $R_{j}(t)$ removeds at time $t$ on patch $j$. Notice that the population on patch $j$ at time $t$ is $N_{j}(t)=S_{j}(t)+I_{j}(t)+R_{j}(t)$. Assume, for $1 \leq j \leq n$, that the population on patch $j$ is mixing homogeneously and that every individual has the same average number of contacts $\beta_{j}>0$ per unit time, where $\beta_{j}$ is a constant. The assumptions of the last sentence will lead to a standard incidence function on patch $j$ (see subsection 8.1.1).

Suppose all new borns on all patches are susceptible - there is no vertical transmission. Suppose further that the birth rate at time $t$ on patch $j$ is a function of the population on patch $j$, namely $b_{j}\left(N_{j}(t)\right)$. Let the birth functions satisfy the following biologically sensible requirement:

$$
b_{j}(0)=0 \quad \text { and } \quad b_{j}(x)>0 \text { for } x>0 \quad \text { where } 1 \leq j \leq n .
$$


There are different ways to model migration [101]. We shall follow an approach used by Arino and van den Driessche [49]. Assume, then, that migration occurs between any pair of patches at the following rates:

- $m_{k, j}^{S}=$ per capita migration rate of susceptibles from patch $k$ to patch $j$

- $m_{k, j}^{I}=$ per capita migration rate of infectives from patch $k$ to patch $j$

- $m_{k, j}^{R}=$ per capita migration rate of removeds from patch $k$ to patch $j$.

These migration rates are all non-negative constants. It is not sensible to think of individuals migrating out of their patch and into it at the same instant, so we set $m_{j, j}^{S}=m_{j, j}^{I}=m_{j, j}^{R}=0$ for $1 \leq j \leq n$. Natural mortality rates are known to vary significantly from one country to another, so there is no reason to assume that the natural mortality rate is the same on all patches. Similarly the disease mortality and recovery rates may be patch-dependent. Define, then, the following parameters:

- $\mu_{j}^{S}=$ per capita death rate of susceptibles and removeds on patch $j$

- $\mu_{j}^{I}=$ per capita death rate of infectives on patch $j$

- $\gamma_{j}=$ per capita recovery rate of infectives on patch $j$

All of these parameters are assumed to be positive constants. Notice that, for $1 \leq j \leq n$, the per capita susceptible death rate on patch $j$ and the per capita removed death rate on patch $j$ are the same, which is reasonable if recovery from the disease does not shorten life expectancy. Assume, for $1 \leq j \leq n$, that $\mu_{j}^{I} \geq \mu_{j}^{S}$ (contracting an infection is seldom beneficial to a creature). Assume that recovery confers permanent immunity. By considering the changes in the numbers of susceptibles, infectives, and removeds on patch $j(1 \leq j \leq n)$ in a short time interval $[t, t+d t]$, and letting $d t \rightarrow 0$ whilst noticing, for example, that $\lim _{d t \rightarrow 0}\left(\frac{S_{j}(t+d t)-S_{j}(t)}{d t}\right)=\frac{d S_{j}(t)}{d t}$, our hypotheses lead to the following model:

$$
\begin{aligned}
\frac{d S_{j}}{d t} & =b_{j}\left(N_{j}\right)-\beta_{j} \frac{S_{j} I_{j}}{N_{j}}-\mu_{j}^{S} S_{j}+\left(\sum_{k=1}^{n} m_{k, j}^{S} S_{k}\right)-\sum_{k=1}^{n} m_{j, k}^{S} S_{j} \\
\frac{d I_{j}}{d t} & =\beta_{j} \frac{S_{j} I_{j}}{N_{j}}-\left(\gamma_{j}+\mu_{j}^{I}\right) I_{j}+\left(\sum_{k=1}^{n} m_{k, j}^{I} I_{k}\right)-\sum_{k=1}^{n} m_{j, k}^{I} I_{j} \\
\frac{d R_{j}}{d t} & =\gamma_{j} I_{j}-\mu_{j}^{S} R_{j}+\left(\sum_{k=1}^{n} m_{k, j}^{R} R_{k}\right)-\sum_{k=1}^{n} m_{j, k}^{R} R_{j}
\end{aligned}
$$

for $t>0$ and for $1 \leq j \leq n$, where we use the shorthand $S_{j}, I_{j}, R_{j}$, and $N_{j}$ for $S_{j}(t)$, $I_{j}(t), R_{j}(t)$, and $N_{j}(t)$ respectively.

Equations (9.2), (9.3), and (9.4) represent a model without vaccinations for the spread of the disease across the $n$ patches. For a sensible model we require initial data. Therefore assume that

$$
S_{j}(0) \geq 0, \quad I_{j}(0) \geq 0, \quad R_{j}(0) \geq 0, \quad S_{j}(0)+I_{j}(0)+R_{j}(0)>0 \quad \text { for } 1 \leq j \leq n .
$$

We must have $I_{j}(0)>0$ for at least one value of $j$, for otherwise the disease is already absent from all $n$ patches at the initial time $t=0$.

Suppose that a pulse vaccination strategy (PVS) is introduced onto each patch according to the following definition: 
Definition 9.1. On patch $j$, for $1 \leq j \leq n$, pulses occur every $T_{j}>0$ time units, where $T_{j}$ is constant. The first pulse occurs at time $t_{1, j}>0$ and, for $i \geq 2$, the $i$-th pulse occurs at $t_{i, j}=t_{1, j}+(i-1) T_{j}$. Each pulse instantaneously transfers a fixed proportion $p_{j}$ (where $\left.0<p_{j}<1\right)$ of susceptibles in patch $j$ to the removed class in patch $j$. Thus $S_{j}\left(t_{i, j}\right)=$ $\left(1-p_{j}\right) S_{j}\left(t_{i, j}^{-}\right)$and $R_{j}\left(t_{i, j}\right)=R_{j}\left(t_{i, j}^{-}\right)+p_{j} S_{j}\left(t_{i, j}^{-}\right)$where $t_{i, j}^{-}$is the time "momentarily" before time $t_{i, j}$. Between pulses, the system on patch $j$ evolves according to equations (9.2), (9.3), and (9.4), and the system begins with the initial data (9.5). The birth functions are assumed to satisfy (9.1). Let $q_{j}=1-p_{j}$.

The model with a pulse vaccination strategy on each patch therefore consists of a series of infinitely many initial value problems (IVPs), with the initial time of the $(i+1)$-th IVP being the time of the first pulse that occurs strictly later than the initial time of the $i$-th IVP. At the initial time of the $i$-th IVP, it is possible that pulses occur simultaneously on more than one patch.

For both of the models - without vaccination or with pulse vaccination strategies - it is easy to deduce from standard results that a unique solution exists for $t>0$. It is also straightforward to deduce from known results (p. 81, [128]) that, given our assumptions on the initial data in (9.5) and given (9.1), then $S_{j}(t) \geq 0, I_{j}(t) \geq 0$, $R_{j}(t) \geq 0$, and $N_{j}(t)>0$ for $t>0$ for $1 \leq j \leq n$. These properties are collectively known as positivity.

\subsection{Natural extinction and endemicity}

If it is known that a particular disease will die out naturally, public health authorites may decide not to implement a vaccination program against it. Of course a disease which is dying out may still claim some victims, but the resources of a public health authority are limited and fatal diseases that are endemic are likely to be of greater priority than diseases that will die out by themselves. Therefore, before constructing successful pulse vaccination strategies in the SIR model defined in the last section, it is sensible to have an understanding of when the disease will die out naturally and when it will persist naturally.

Theorem 9.1. Consider the model defined by (9.1), (9.2), (9.3), (9.4), and (9.5). Define $\hat{\beta}=\max _{1 \leq j \leq n}\left\{\beta_{j}\right\}$ and $\theta=\min _{1 \leq j \leq n}\left\{\gamma_{j}+\mu_{j}^{I}\right\}$. If $\hat{\beta}<\theta$, then $I_{j}(t) \rightarrow 0$ as $t \rightarrow \infty$ for $1 \leq j \leq n$.

Proof. Add together all $n$ equations defined by (9.3) for $1 \leq j \leq n$ to obtain:

$$
\frac{d}{d t}\left(\sum_{j=1}^{n} I_{j}(t)\right)=\sum_{j=1}^{n}\left(\beta_{j} \frac{S_{j}(t) I_{j}(t)}{N_{j}(t)}-\left[\gamma_{j}+\mu_{j}^{I}\right] I_{j}(t)\right) .
$$

By positivity we know that $\frac{S_{j}(t)}{N_{j}(t)} \leq 1$ for $t>0$ and where $1 \leq j \leq n$. But then, given the definitions of $\hat{\beta}$ and $\theta$ in the statement of the theorem, we can write:

$$
\frac{d I(t)}{d t} \leq(\hat{\beta}-\theta) I(t) \text { for } t>0,
$$


where $I(t)=\sum_{j=1}^{n} I_{j}(t)$. Hence (using theorem 1.1, pp. 78-79, [128]) we know that $I(t) \leq I^{*}(t)$ for $t \geq 0$ where $I^{*}(0)=I(0)>0$ and where $\frac{d I^{*}(t)}{d t}=(\hat{\beta}-\theta) I^{*}(t)$ for $t>0$. Solving for $I^{*}(t)$ and using positivity of $I_{j}(t)$, we then have, for $1 \leq j \leq n$ :

$$
0 \leq I_{j}(t) \leq I(t) \leq I^{*}(t)=I(0) e^{(\hat{\beta}-\theta) t} \text { for } t>0 .
$$

Combining (9.8) with the assumption that $\hat{\beta}-\theta<0$ immediately yields that $I_{j}(t) \rightarrow 0$ as $t \rightarrow \infty$ for $1 \leq j \leq n$, as required.

Rigorous proofs of endemicity in metapopulation models are, at present, scarce. However, disease persistence in an SIS model has been established analytically by Jin and Wang [88]. Also, simulations demonstrating endemicity in a two-patch SEIR model for a non-fatal disease have been carried out by Arino et al [48]. We shall now demonstrate by simulation that the disease can remain endemic in the model of (9.2), (9.3), (9.4), and (9.5) when there are two patches.

In figure 9.1 we simulate the model of (9.2), (9.3), and (9.4) when there are $n=2$ patches and where the parameters are chosen as follows:

$$
\begin{array}{llll}
\beta_{1}=12 & \mu_{1}^{S}=1 & \mu_{1}^{I}=1.1 & \gamma_{1}=0.9 \\
\beta_{2}=15 & \mu_{2}^{S}=1.1 & \mu_{2}^{I}=1.2 & \gamma_{2}=1.2 .
\end{array}
$$

The migration rates are:

$$
\begin{array}{lll}
m_{1,2}^{S}=1 & m_{1,2}^{I}=0.8 & m_{1,2}^{R}=1 \\
m_{2,1}^{S}=1.2 & m_{2,1}^{I}=1 & m_{2,1}^{R}=1.2 .
\end{array}
$$

The initial conditions are $\left(S_{1}(0), I_{1}(0), R_{1}(0)\right)=(10,8,0)$ and $\left(S_{2}(0), I_{2}(0), R_{2}(0)\right)=$ $(13,7,10)$. (The population unit may be thousands of individuals so it is not necessary to worry about stochastic effects from apparently low initial conditions.) The birth functions in figure 9.1 are both linear: on patch 1 we have $b_{1}\left(N_{1}\right)=N_{1}$ and on patch 2 we have $b_{2}\left(N_{2}\right)=2 N_{2}$.

Figure 9.2 differs from figure 9.1 only in the choices of the birth functions. Thus in figure 9.2 the birth functions are both Nicholson (see subsection 3.2.1): on patch 1 we have $b_{1}\left(N_{1}\right)=3 N_{1} e^{-0.03 N_{1}}$ and on patch 2 we have $b_{2}\left(N_{2}\right)=4 N_{2} e^{-0.09 N_{2}}$.

Figure 9.3 differs from figures 9.1 and 9.2 only in the choice of the contact rates and the birth functions. Thus the contact rates are now $\beta_{1}=1.2$ and $\beta_{2}=6.5$ and the birth functions are both Allee (see subsection 3.2.1): on patch 1 we have $b_{1}\left(N_{1}\right)=$ $1.5 N_{1}^{2} e^{-0.05 N_{1}}$ and on patch 2 we have $b_{2}\left(N_{2}\right)=2 N_{2}^{2} e^{-0.1 N_{2}}$.

Figures 9.1, 9.2, and 9.3 show that the disease can remain endemic when both birth functions are linear, Nicholson, and Allee respectively. Certainly, then, there appear to be grounds for seeking vaccination strategies to eradicate the disease.

\subsection{Pulse vaccination strategies}

We have seen evidence in the previous section that the disease can remain endemic in the model of (9.2), (9.3), (9.4), and (9.5). In this section we seek conditions on the 

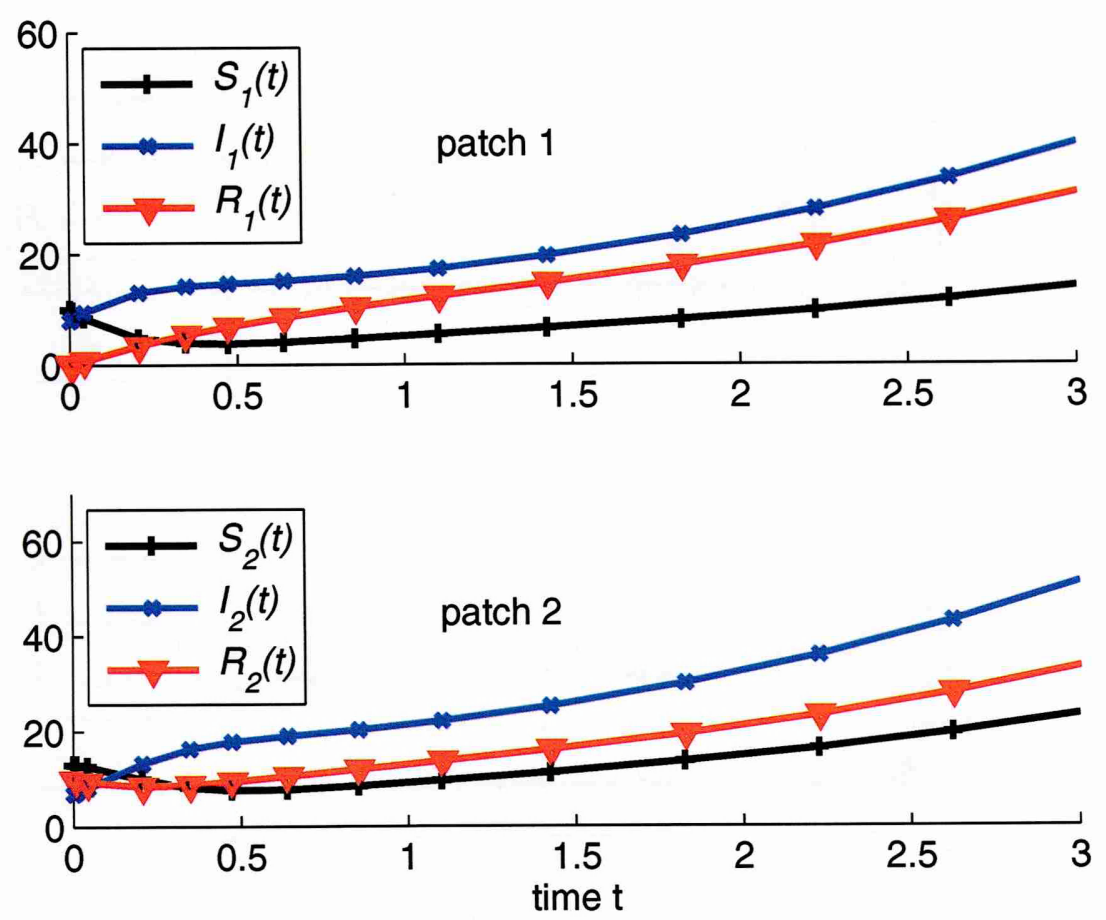

Figure 9.1: Natural endemicity on two patches. The birth function is linear on both patches. See section 9.3 for parameter choices and comments. 

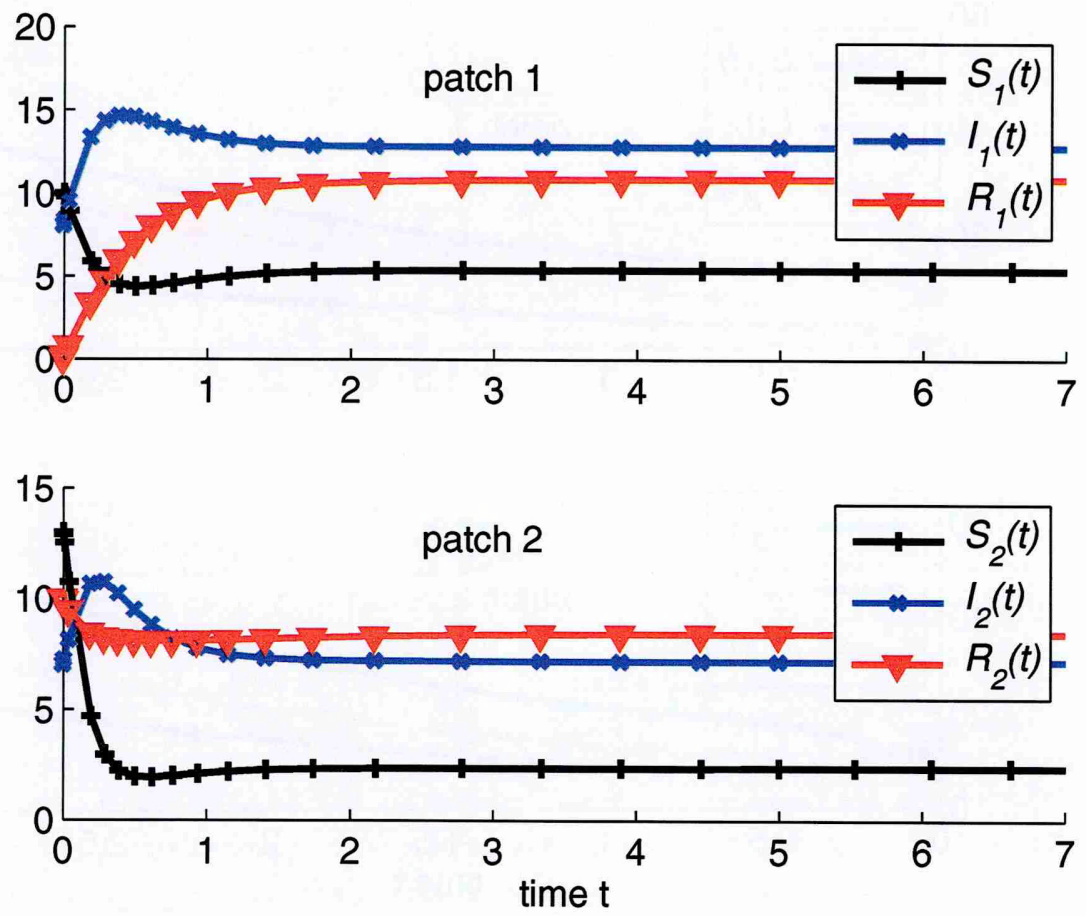

Figure 9.2: Natural endemicity on two patches. The birth function is of Nicholson type on both patches. See section 9.3 for parameter choices and comments. 

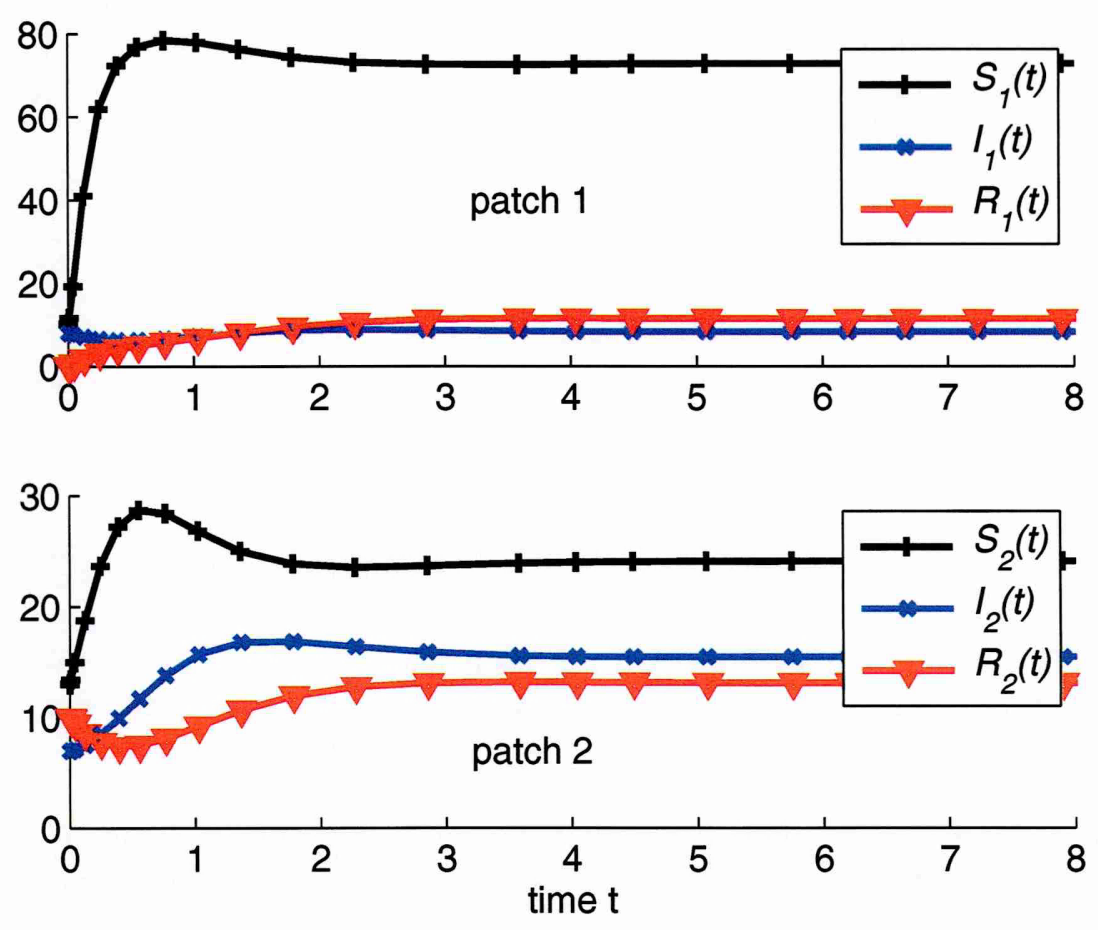

Figure 9.3: Natural endemicity on two patches. The birth function is of Allee type on both patches. See section 9.3 for parameter choices and comments. 
pulse vaccination strategies such that the disease will be eradicated on all $n$ patches simultaneously whilst asking if the PVS parameters on every patch can be chosen with some automony along the lines stated in the introduction.

Although many human populations have been growing in recent decades and centuries (see $[34,105]$ and table 8.1), this growth is unlikely to continue forever. Also, despite dire predictions by numerous gloomy individuals, there has not been a major global human population crash for many centuries. It seems reasonable to eventually hope for some sort of stability in human populations. The reason we mention these details is because our method of deriving successful pulse vaccination strategies assumes that our subpopulations are stable. We begin with a lemma in which we bound above the susceptible, infective, and removed populations on every patch.

Lemma 9.1. Consider the PVS model described in definition 9.1. Suppose that the birth functions are bounded above, that is, suppose, for $1 \leq j \leq n$, that $\max _{N_{j} \geq 0}\left\{b_{j}\left(N_{j}\right)\right\} \leq M_{j}$ where $M_{j}$ is a positive constant. Also let $N(0)=\sum_{j=1}^{n} N_{j}(0)$. Then $S_{j}(t) \leq \frac{3 M}{2 d}$ and $I_{j}(t) \leq \frac{3 M}{2 d}$ and $R_{j}(t) \leq \frac{3 M}{2 d}$ for $t>t^{*}$ where:

$$
\begin{aligned}
M & =\sum_{j=1}^{n} M_{j}, \\
d & =\min _{1 \leq j \leq n}\left\{\mu_{j}^{S}, \mu_{j}^{I}\right\}=\min _{1 \leq j \leq n}\left\{\mu_{j}^{S}\right\}
\end{aligned}
$$

and where, if $N(0) \leq \frac{M}{d}$, then $t^{*}=0$, but if $N(0)>\frac{M}{d}$ then

$$
t^{*}=\max \left\{0,-\frac{1}{d} \ln \left(\frac{M}{2 d\left(N(0)-\frac{M}{d}\right)}\right)\right\} \text {. }
$$

The assumption on the birth functions in lemma 9.1 is sensible because, in real life, a birth rate will never be infinite. Notice that the number $t^{*}$ mentioned in lemma 9.1 will be used in theorem 9.3 later on. Here is the proof of the lemma:

Proof. If we add together all $3 n$ equations defined by (9.2), (9.3), and (9.4) for $1 \leq j \leq$ $n$, and bear in mind that $S_{j}(t)+I_{j}(t)+R_{j}(t)=N_{j}(t)$, we obtain, for $t>0$ :

$$
\frac{d}{d t}\left(\sum_{j=1}^{n} N_{j}(t)\right)=\sum_{j=1}^{n}\left(b_{j}\left(N_{j}(t)\right)-\left[\mu_{j}^{S} S_{j}(t)+\mu_{j}^{I} I_{j}(t)+\mu_{j}^{S} R_{j}(t)\right]\right)
$$

Equation (9.15) holds both for the model without vaccinations and for the PVS model. This is because in the PVS model, the model equations are the same between pulses as in the model without vaccinations and each instantaneous pulse on any patch does not alter the total size of the population on that or any other patch.

Let $N(t)=\sum_{j=1}^{n} N_{j}(t)$. Then using the definition of $d$ given in the statement of the lemma, as well as (9.15), we can write:

$$
\frac{d N(t)}{d t} \leq\left(\sum_{j=1}^{n} b_{j}\left(N_{j}(t)\right)\right)-d N(t)
$$


Since $N_{j}(t) \geq 0$ for all $t \geq 0$ by positivity and using 9.1 , we can deduce by (9.16) that:

$$
\frac{d N(t)}{d t} \leq M-d N(t) \text { for } t>0
$$

where $M=\sum_{j=1}^{n} M_{j}$.

It follows by theorem 1.1 on pages 78-79 of [128] that $N(t) \leq N^{*}(t)$ for $t>0$ where $N^{*}(0)=N(0)=\sum_{j=1}^{n} N_{j}(0)>0$ and where, for $t>0$, we have $\frac{d N^{*}(t)}{d t}=M-d N^{*}(t)$. Solving for $N^{*}(t)$ reveals that:

$$
N(t) \leq N^{*}(t)=\frac{M}{d}+\left(N(0)-\frac{M}{d}\right) e^{-d t} \text { for } t>0 .
$$

By positivity we know that $0 \leq S_{j}(t) \leq N_{j}(t) \leq N(t)$ for $t \geq 0$. Further, since $e^{-d t} \rightarrow 0$ as $t \rightarrow \infty$, we can deduce by (9.18) that, for all $t$ large enough, then

$$
S_{j}(t) \leq N_{j}(t) \leq N(t) \leq W \text { for } 1 \leq j \leq n,
$$

where $W=\frac{3 M}{2 d}$. In fact (9.19) can be seen to hold for all $t>t^{*}$ where $t^{*}$ is defined in the statement of lemma 9.1. Similarly we may bound above $I_{j}(t)$ and $R_{j}(t)$.

In our next result, we find that the pulse vaccination strategies will succeed provided they keep the proportion of susceptibles on every patch suitably small for all time large enough.

Theorem 9.2. In the PVS model described in definition 9.1, suppose that the birth functions are bounded above. Also, suppose that, for $1 \leq j \leq n$, the PVS on patch $j$ ensures that, for all $t$ big enough (for all $t>t_{j}^{*}$, say), then:

$$
\beta_{j} \frac{S_{j}(t)}{N_{j}(t)}<\frac{\gamma_{j}+\mu_{j}^{I}}{2}
$$

Then $I_{j}(t) \rightarrow 0$ as $t \rightarrow \infty$ for $1 \leq j \leq n$.

Proof. Let $\bar{t}=\max _{1 \leq j \leq n}\left\{t_{j}^{*}\right\}$. Vaccination pulses do not make the infective population on any patch change impulsively. By (9.6) and (9.20), we can say, then, for all $t>\bar{t}$, that:

$$
\frac{d}{d t}\left(\sum_{j=1}^{n} I_{j}(t)\right) \leq-\sum_{j=1}^{n}\left(\frac{\gamma_{j}+\mu_{j}^{I}}{2}\right) I_{j}(t) .
$$

Let $I(t)=\sum_{j=1}^{n} I_{j}(t)$. Let $-\alpha=\max _{1 \leq j \leq n}\left\{-\frac{\gamma_{j}+\mu_{j}^{I}}{2}\right\}$. Notice that $\alpha>0$. Then for $t>\bar{t}$, we have, by (9.21),

$$
\frac{d I(t)}{d t} \leq-\alpha I(t)
$$

Hence (using theorem 1.1, pp. 78-79, [128]) we know that $I(t) \leq I^{*}(t)$ for $t \geq \bar{t}$ where $I^{*}(\bar{t})=I(\bar{t}) \geq 0$ and where $\frac{d I^{*}(t)}{d t}=-\alpha I^{*}(t)$ for $t>\bar{t}$. Solving for $I^{*}(t)$ and using positivity, we then have, for $1 \leq j \leq n$ :

$$
0 \leq I_{j}(t) \leq I(t) \leq I^{*}(t)=I(\bar{t}) e^{-\alpha(t-\bar{t})} \text { for } t \geq \bar{t} .
$$


Combining (9.23) with the assumption that $\alpha>0$ immediately yields that $I_{j}(t) \rightarrow$ 0 as $t \rightarrow \infty$ for $1 \leq j \leq n$, as required.

Thus disease eradication will be guaranteed on all patches by the pulse vaccination strategies if the PVS on patch $j$ (for $1 \leq j \leq n$ ) ensures that (9.20) holds for all $t$ large enough. In order to establish a condition on the PVS on patch $j$ such that $(9.20)$ will hold for all $t$ large enough, we must first prove another lemma in which we bound below our subpopulations.

Lemma 9.2. Consider the PVS model of definition 9.1. For $1 \leq j \leq n$, define:

$$
D_{j}=\max \left\{\mu_{j}^{S}+\sum_{k=1}^{n} m_{j, k}^{S}, \quad \mu_{j}^{I}+\sum_{k=1}^{n} m_{j, k}^{I}, \quad \mu_{j}^{S}+\sum_{k=1}^{n} m_{j, k}^{R}\right\} .
$$

(A1) If the birth function on patch $j$ is of Nicholson type (see subsection 3.2.1) with $b_{j}\left(N_{j}\right)=$ $\lambda_{1, j} N_{j} e^{-\lambda_{2, j} N_{j}}$, and if $D_{j}<\lambda_{1, j}$, then $b_{j}\left(N_{j}\right)=D_{j} N_{j}$ has a unique positive solution $N_{j}^{*}$ and $N_{j}(t)>\frac{N_{j}^{*}}{2}$ for all $t$ large enough.

(A2) If the birth function on patch $j$ is of Allee type (see subsection 3.2.1) with $b_{j}\left(N_{j}\right)=$ $\alpha_{1, j} N_{j}^{2} e^{-\alpha_{2, j} N_{j}}$, and if $D_{j}<\frac{\alpha_{1, j}}{e \alpha_{2, j}}$, then $b_{j}\left(N_{j}\right)=D_{j} N_{j}$ has two positive solutions, which we may label $N_{1, j}^{*}$ and $N_{2, j}^{*}$, with $N_{1, j}^{*}<N_{2, j}^{*}$. If $N_{j}(0)>N_{1, j}^{*}$ then $N_{j}(t)>$ $N_{1, j}^{*}$ for $t>0$ and $N_{j}(t)>\frac{N_{1, j}^{*}+N_{2, j}^{*}}{2}$ for all $t$ large enough.

Proof. Bearing in mind that $N_{j}(t)=S_{j}(t)+I_{j}(t)+R_{j}(t)$ and that $N_{j}(t)$ is not impulsively changed when any vaccination pulse occurs on any patch, we add together (9.2), (9.3), and (9.4) to obtain, for $t>0$ :

$$
\begin{aligned}
& \frac{d N_{j}}{d t}=b_{j}\left(N_{j}\right)+\left(\sum_{k=1}^{n} m_{k, j}^{S} S_{k}\right)+\left(\sum_{k=1}^{n} m_{k, j}^{I} I_{k}\right)+\left(\sum_{k=1}^{n} m_{k, j}^{R} R_{k}\right) \\
& -\left(\mu_{j}^{S}+\sum_{k=1}^{n} m_{j, k}^{S}\right) S_{j}-\left(\mu_{j}^{I}+\sum_{k=1}^{n} m_{j, k}^{I}\right) I_{j}-\left(\mu_{j}^{S}+\sum_{k=1}^{n} m_{j, k}^{R}\right) R_{j} .
\end{aligned}
$$

By positivity we have:

$$
\left(\sum_{k=1}^{n} m_{k, j}^{S} S_{k}\right)+\left(\sum_{k=1}^{n} m_{k, j}^{I} I_{k}\right)+\left(\sum_{k=1}^{n} m_{k, j}^{R} R_{k}\right) \geq 0,
$$

and:

$$
\begin{aligned}
& -\left(\mu_{j}^{S}+\sum_{k=1}^{n} m_{j, k}^{S}\right) S_{j}-\left(\mu_{j}^{I}+\sum_{k=1}^{n} m_{j, k}^{I}\right) I_{j}-\left(\mu_{j}^{S}+\sum_{k=1}^{n} m_{j, k}^{R}\right) R_{j} \\
& \geq \quad-D_{j} S_{j}-D_{j} I_{j}-D_{j} R_{j}=-D_{j} N_{j} .
\end{aligned}
$$

Using (9.25), (9.26), and (9.27), we can write:

$$
\frac{d N_{j}}{d t} \geq b_{j}\left(N_{j}\right)-D_{j} N_{j} .
$$


It follows (using theorem 1.1, pp. 78-79, [128]) that $N_{j}(t) \geq N_{j}^{*}(t)$ for $t \geq 0$ where $N_{j}^{*}(0)=N_{j}(0)$ and where, for $t>0$, we have $\frac{d N_{j}^{*}}{d t}=b_{j}\left(N_{j}^{*}\right)-D_{j} N_{j}^{*}$. Results (A1) and (A2) of the lemma now follow trivially.

By lemma 9.2, we know that there will be circumstances under which the population on patch $j(1 \leq j \leq n)$ will remain bounded below. We have also commented after the statement of lemma 9.1 how, in real life, a birth rate will never be infinite. In particular, it is sensible to expect $\max _{N_{j} \geq 0}\left\{\frac{b_{j}\left(N_{j}\right)}{N_{j}}\right\}$ to be finite, and indeed if $b_{j}$ is any of the three birth functions described in subsection 3.2.1 then such an expectation holds. Thus we can be sure that the assumptions made in the following theorem are sensible:

Theorem 9.3. Consider the PVS model of definition 9.1. As in lemma 9.1, assume, for $1 \leq j \leq n$, that $\max _{N_{j} \geq 0}\left\{b_{j}\left(N_{j}\right)\right\} \leq M_{j}$ where $M_{j}$ is a positive constant. Suppose also that $\max _{N_{j} \geq 0}\left\{\frac{b_{j}\left(N_{j}\right)}{N_{j}}\right\} \leq K_{j}$ for some positive constant $K_{j}$ and that $N_{j}(t)>H_{j}$ for all time large enough (for all $t>t_{j}^{*}$, say).

Let $M=\sum_{j=1}^{n} M_{j}$ and $d=\min _{1 \leq j \leq n}\left\{\mu_{j}^{S}, \mu_{j}^{I}\right\}=\min _{1 \leq j \leq n}\left\{\mu_{j}^{S}\right\}$. Also let

$$
L_{j}=K_{j}+\left(\frac{3 M}{2 d}\right)\left(\frac{1}{H_{j}}\right)\left(\sum_{k=1}^{n} m_{k, j}^{S}\right)+2 \mu_{j}^{S}+\mu_{j}^{I}+\sum_{k=1}^{n}\left[m_{j, k}^{S}+m_{j, k}^{I}+m_{j, k}^{R}\right]
$$

Assume, for $1 \leq j \leq n$, that the PVS on patch $j$ satisfies the relationship:

$$
\frac{L_{j} T_{j}}{1-q_{j}}<\frac{\gamma_{j}+\mu_{j}^{I}}{2 \beta_{j}}
$$

Then there exists $t_{j}>0$ such that, for all $t>t_{j}$, the PVS on patch $j$ will ensure that $\beta_{j} \frac{S_{j}(t)}{N_{j}(t)}<\frac{\gamma_{j}+\mu_{j}^{I}}{2}$ (and therefore theorem 9.2 applies).

Proof. First note by the quotient rule for differentiation that:

$$
\frac{d}{d t}\left(\frac{S_{j}}{N_{j}}\right)=\frac{\frac{d S_{j}}{d t}}{N_{j}}-\frac{S_{j}}{N_{j}}\left(\frac{\frac{d N_{j}}{d t}}{N_{j}}\right) \quad \text { for } t>0, \quad t \neq t_{i, j} \text { where } i \geq 1,
$$

where (recall by definition 9.1) $t_{i, j}$ is the time of the $i$-th pulse on patch $j$.

Now we already have an expression for $\frac{d S_{j}}{d t}$ in (9.2) and an expression for $\frac{d N_{j}}{d t}$ is given in (9.25).

By positivity we know that $-\beta_{j} \frac{S_{j} I_{j}}{N_{j}}-\sum_{k=1}^{n} m_{j, k}^{S} S_{j} \leq 0$ and $N_{j}(t)>0$ for $t>0$. By (9.2) we may then bound above $\left(\frac{d S_{j}}{N_{j}}\right)$ as follows:

$$
\frac{\frac{d S_{j}}{d t}}{N_{j}} \leq \frac{b_{j}\left(N_{j}\right)}{N_{j}}+\sum_{k=1}^{n} m_{k, j}^{S}\left(\frac{S_{k}}{N_{j}}\right) \quad \text { for } t>0, \quad t \neq t_{i, j} \text { where } i \geq 1
$$


But by assumption $\max _{N_{j} \geq 0}\left\{\frac{b_{j}\left(N_{j}\right)}{N_{j}}\right\} \leq K_{j}$ and, for $t>t_{j}^{*}$, then $\frac{1}{N_{j}(t)}<\frac{1}{H_{j}}$. Also, by lemma 9.1, we know that $S_{j}<\frac{3 M}{2 d}$ for $t>t^{*}$ where $t^{*}$ is defined in the statement of lemma 9.1. Let $t_{j}^{* *}=\max \left\{t^{*}, t_{j}^{*}\right\}$. Then, by (9.32), we know that:

$$
\frac{\frac{d S_{j}}{d t}}{N_{j}} \leq K_{j}+\left(\frac{3 M}{2 d}\right)\left(\frac{1}{H_{j}}\right) \sum_{k=1}^{n} m_{k, j}^{S} \quad \text { for } t>t_{j}^{* *}, \quad t \neq t_{i, j} \text { where } i \geq 1 .
$$

Again, by positivity we know, for $t>0$, that

$$
-b_{j}\left(N_{j}\right)-\left(\sum_{k=1}^{n} m_{k, j}^{S} S_{k}\right)-\left(\sum_{k=1}^{n} m_{k, j}^{I} I_{k}\right)-\left(\sum_{k=1}^{n} m_{k, j}^{R} R_{k}\right) \leq 0 .
$$

We also know that $0 \leq \frac{S_{j}}{N_{j}}, \frac{I_{j}}{N_{j}}, \frac{R_{j}}{N_{j}} \leq 1$. But then:

$$
\begin{aligned}
& \left(\mu_{j}^{S}+\sum_{k=1}^{n} m_{j, k}^{S}\right) \frac{S_{j}}{N_{j}}+\left(\mu_{j}^{I}+\sum_{k=1}^{n} m_{j, k}^{I}\right) \frac{I_{j}}{R_{j}}+\left(\mu_{j}^{S}+\sum_{k=1}^{n} m_{j, k}^{R}\right) \frac{R_{j}}{N_{j}} \\
& \leq 2 \mu_{j}^{S}+\mu_{j}^{I}+\sum_{k=1}^{n}\left[m_{j, k}^{S}+m_{j, k}^{I}+m_{j, k}^{R}\right] .
\end{aligned}
$$

Therefore, using the fact that $0 \leq \frac{S_{j}}{N_{j}} \leq 1$, and also using (9.25), we may bound above $-\frac{S_{j}}{N_{j}}\left(\frac{d N_{j}}{\frac{d t}{N_{j}}}\right)$ for $t>0$ as follows:

$$
-\frac{S_{j}}{N_{j}}\left(\frac{\frac{d N_{j}}{d t}}{N_{j}}\right) \leq 2 \mu_{j}^{S}+\mu_{j}^{I}+\sum_{k=1}^{n}\left[m_{j, k}^{S}+m_{j, k}^{I}+m_{j, k}^{R}\right] .
$$

Combining (9.33) and (9.34) with (9.31) reveals that:

$$
\frac{d}{d t}\left(\frac{S_{j}}{N_{j}}\right) \leq L_{j} \quad \text { for } t>t_{j}^{* *}, \quad t \neq t_{i, j} \text { where } i \geq 1,
$$

where $L_{j}$ is a positive constant defined in (9.29).

Let $x_{j}(t)=\frac{S_{j}(t)}{N_{j}(t)}$. We know that pulses occur on patch $j$ at times $t_{i, j}$ as outlined in definition 9.1. Let $t_{u_{j}, j}$ be the time of the first cull on patch $j$ to occur strictly later than $t_{j}^{* *}$. Then we can write, by $(9.35)$ :

$$
\frac{d x_{j}(t)}{d t} \leq L_{j} \text { for } t>t_{u_{j}, j} \text { where } t \neq t_{i, j} \text { for } i \geq u_{j}
$$

Then (using theorem 1.1, pp. 78-79, [128]), we can say, for any $i \geq u_{j}$, that $x_{j}(t) \leq$ $g_{j}(t)$ for $t \in\left[t_{i, j}, t_{i+1, j}\right)$ where $g_{j}\left(t_{i, j}\right)=x_{j}\left(t_{i, j}\right)$ and where:

$$
\frac{d g_{j}(t)}{d t}=L_{j} \text { for } t \in\left(t_{i, j}, t_{i+1, j}\right) .
$$

Solving for $g_{j}(t)$ on $\left[t_{i, j}, t_{i+1, j}\right)$ reveals that:

$$
x_{j}(t) \leq g_{j}(t)=L_{j}\left(t-t_{i, j}\right)+x_{j}\left(t_{i, j}\right) \quad \text { for } i \geq u_{j} .
$$


But then:

$$
x_{j}\left(t_{i+1, j}^{-}\right) \leq L_{j} T_{j}+x_{j}\left(t_{i, j}\right),
$$

where $t_{i+1, j}^{-}$denotes the time "momentarily" before the time $t_{i+1, j}$.

But now $x_{j}\left(t_{i+1, j}\right)=q_{j} x_{j}\left(t_{i+1, j}^{-}\right)$since, by definition 9.1, $S_{j}\left(t_{i+1, j}\right)=q_{j} S_{j}\left(t_{i+1, j}^{-}\right)$ and $N_{j}\left(t_{i+1, j}^{-}\right)=N_{j}\left(t_{i+1, j}\right)$. Hence by (9.39):

$$
x_{j}\left(t_{i+1, j}\right)=q_{j} x_{j}\left(t_{i+1, j}^{-}\right) \leq q_{j} L_{j} T_{j}+q_{j} x_{j}\left(t_{i, j}\right) .
$$

Let $z_{i}=x_{j}\left(t_{i, j}\right)$. Then by (9.40):

$$
z_{i+1} \leq A+q_{j} z_{i} \quad \text { for } i \geq u_{j},
$$

where $A=q_{j} L_{j} T_{j}>0$. Iterating (9.41) we quickly find that, for $i \geq 1$ :

$$
z_{u_{j}+i} \leq q_{j}^{i} z_{u_{j}}+A \sum_{r=0}^{i-1} q_{j}^{r} .
$$

Now $0<q_{j}<1$, so the partial sum in (9.42) will be less than the entire sum, that is,

$$
\sum_{r=0}^{i-1} q_{j}^{r}<\frac{1}{1-q_{j}} .
$$

Hence:

$$
z_{u_{j}+i} \leq q_{j}^{i} z_{u_{j}}+A \frac{1}{1-q_{j}} \text { for } i \geq 1 .
$$

But using lemma 9.1 and the assumption in the theorem that $N_{j}(t)>H_{j}$ for $t>t_{j}^{*}$, we know that:

$$
z_{u_{j}}=x_{j}\left(t_{u_{j}, j}\right)=\frac{S_{j}\left(t_{u_{j}, j}\right)}{N_{j}\left(t_{u_{j}, j}\right)} \leq \frac{\left(\frac{3 M}{2 d}\right)}{H_{j}} .
$$

Therefore:

$$
x_{j}\left(t_{u_{j}+i, j}\right)=z_{u_{j}+i} \leq \frac{3 M q_{j}^{i}}{2 d H_{j}}+\frac{q_{j} L_{j} T_{j}}{1-q_{j}} \text { for } i \geq 1 .
$$

But then by (9.38) we have, for $t \in\left[t_{u_{j}+i, j}, t_{u_{j}+i+1, j}\right)$ for $i \geq 1$, that:

$$
\begin{aligned}
\frac{S_{j}(t)}{N_{j}(t)}=x_{j}(t) & \leq L_{j} T_{j}+\frac{3 M q_{j}^{i}}{2 d H_{j}}+\frac{q_{j} L_{j} T_{j}}{1-q_{j}} \\
& =\frac{L_{j} T_{j}}{1-q_{j}}+\frac{3 M q_{j}^{i}}{2 d H_{j}} .
\end{aligned}
$$

Now inequality (9.30) is true by assumption. But then by (9.47):

$$
\frac{S_{j}(t)}{N_{j}(t)}<\frac{\gamma_{j}+\mu_{j}^{I}}{2 \beta_{j}}
$$

for $t \in\left[t_{u_{j}+i, j}, t_{u_{j}+i+1, j}\right)$ for all $i$ large enough that

$$
\frac{3 M q_{j}^{i}}{2 d H_{j}}<\frac{\gamma_{j}+\mu_{j}^{I}}{2 \beta_{j}}-\frac{L T_{j}}{1-q_{j}}
$$


By (9.30), the right hand side of (9.49) is positive. Thus, since $0<q_{j}<1$, it is clear that (9.49) will hold for all $i$ large enough. Thus, if we let $i_{j}^{*}$ be the least $i$ such that (9.49) holds, then by $(9.48)$ we have:

$$
\frac{S_{j}(t)}{N_{j}(t)} \leq \frac{\gamma_{j}+\mu_{j}^{I}}{2 \beta_{j}} \text { for } t \geq t_{u_{j}+i_{j}^{*}, j}
$$

The following corollary is true immediately by the results in this section:

Corollary 9.1. Make the assumptions, for each $j$ with $1 \leq j \leq n$, in lemma 9.1, lemma 9.2, theorem 9.2, and theorem 9.3. Then $I_{j}(t) \rightarrow 0$ as $t \rightarrow \infty$ for $1 \leq j \leq n$. In other words, the pulse vaccination strategies will eradicate the disease on all patches simultaneously.

\subsection{Regime existence}

It is trivially seen that (9.30) will hold for a sufficiently strong PVS on patch $j$. After all, for any vaccination strength $q_{j}$ satisfying $0<q_{j}<1$, then (9.30) will hold provided the pulses on patch $j$ occur often enough that:

$$
T_{j}<\frac{\left(\gamma_{j}+\mu_{j}^{I}\right)\left(1-q_{j}\right)}{2 \beta_{j} L_{j}} .
$$

Inequality (9.51) is simply a rearrangement of (9.30). To gain insight into how restrictive condition (9.51) is, we plot "stability" diagrams in figure 9.4. The shaded region in the plots depict the part of the $T_{j}-q_{j}$ parameter space for which (9.51) holds. Thus the shaded region in each plot is where $0<q_{j}<1$ and $0<T_{j}<\frac{\left(\gamma_{j}+\mu_{j}^{I}\right)\left(1-q_{j}\right)}{2 \beta_{j} L_{j}}$. Notice how significantly the stable region is reduced by doubling the contact rate $\beta_{j}$. This is sensible because we would expect a higher contact rate to allow a disease to spread more quickly, so that a stronger vaccination strategy would be needed to control it. Notice also that successful pulse vaccination strategies may exist which lie outside the stable regions, since we have proven (9.51) to be sufficient but not necessary for disease eradication.

Stability diagrams similar to those in this section were included in figure 3.2 in subsection 3.4.4. The diagrams in figure 3.2 depicted the stability of a culling regime for an insect pest. From figure 3.2 we observed in subsection 3.4.4 that the stable region was radically reduced if the birth rate of the pest were doubled. This is analogous to the observation made in the last paragraph on how doubling the contact rate $\beta_{j}$ reduces the stable region for a PVS restricted by (9.51). This should come as no great surprise because the contact rate in an epidemiological model functions as a birth rate parameter for the infective population. The analogy is completed by noting that infectives are, in a sense, pests - the purpose of disease control is to eradicate the infective population.

\subsection{Simulations}

In this section we present simulations in which independent pulse vaccination strategies are carried out when there are two patches. In figure 9.5 we plot the number of 

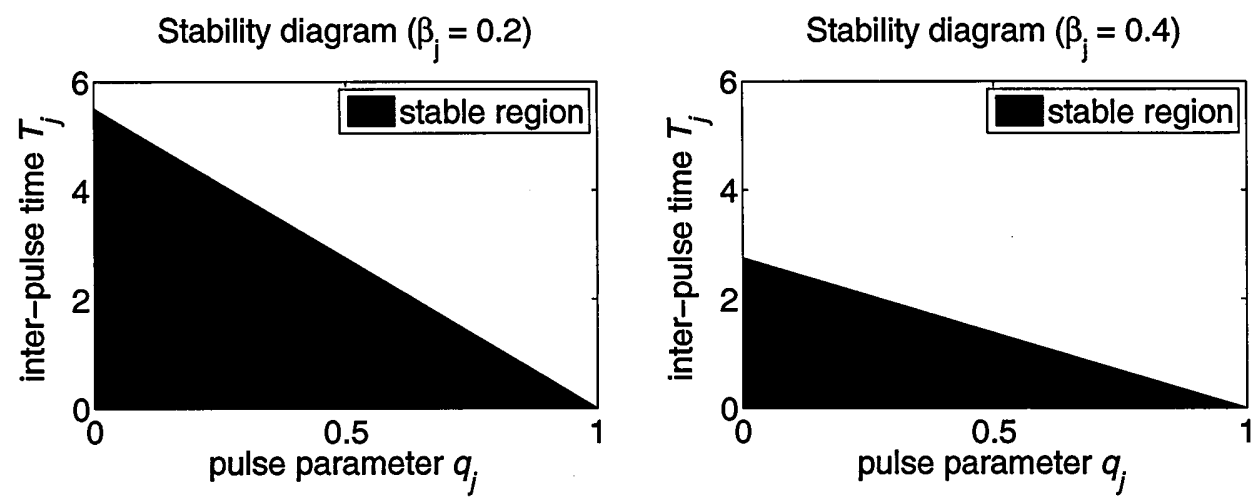

Figure 9.4: Stability diagrams for pulse vaccination strategy on patch $j$, depicting regions in which the strategy is guaranteed to succeed. See section 9.5 for details. In both plots, $L_{j}=1, \gamma_{j}=1.2, \mu_{j}=1$.

infectives on patch 1 and patch 2 by simulating the model of (9.2), (9.3), and (9.4), with the same initial data, model parameters, and birth functions as those used in figure 9.1. A PVS with constant pulse strength $p_{1}=0.95$ (so that $q_{1}=1-p_{1}=0.05$ ) begins on patch 1 at time $t_{1}=3$, with pulses occurring thereafter every $T_{1}=0.2$ time units. A PVS with constant pulse strength $p_{2}=0.9$ (so that $q_{2}=1-p_{2}=0.1$ ) begins on patch 2 at time $t_{2}=3.5$, with pulses occurring thereafter every $T_{2}=0.25$ time units.

The birth functions in figure 9.5 are linear, so theorem 9.3 is not being tested in this simulation because the condition in theorem 9.3 that $\max _{N_{j} \geq 0}\left\{b_{j}\left(N_{j}\right)\right\} \leq M_{j}$ for a positive constant $M_{j}$ for $1 \leq j \leq n$ does not hold. Figure 9.5 is included because it suggests that, in certain circumstances, pulse vaccination strategies will reduce infective populations but only temporarily. The fact that the pulse vaccination strategies in figure 9.5 yield only a temporary reduction in the infective populations could be related to the growth of the total populations on the two patches, as demonstrated by figure 9.1. In figure 9.5, then, we could argue that, as the total populations grow on each patch, the birth functions necessarily increase because they are linear, and this results in an increasing supply of susceptibles to fuel the disease. The pulses on both patches have a diminishing effect as the populations grow because, even though each new pulse will vaccinate a larger number of susceptibles, there will also be a larger number of susceptibles left unvaccinated - pulses are always assumed to vaccinate a fixed proportion of susceptibles.

In view of figure 9.5 and the results of chapter 8 , it may be fruitful to seek conditions under which simultaneous pulse vaccination strategies fail to eradicate a multipatch disease. In particular, if the populations are increasing on every patch, and if the contact rates grow with the populations, can we adapt the methods of chapter 8 to prove that the infective populations will diverge to infinity? This is left as a matter for future research although we make an observation that could assist such research: by reasoning as in lemma 9.2, it is easy to show for our model of (9.2), (9.3), and (9.4), that, if $b_{j}\left(N_{j}\right)=\lambda_{j} N_{j}$ for $1 \leq j \leq n$ and if $\lambda_{j}>D_{j}$ where $D_{j}$ is defined in equation (9.24), then $N_{j}(t) \rightarrow \infty$ as $t \rightarrow \infty$. This will be true whether or not a PVS is carried out on patch $j$ because instantaneous pulses on patch $j$ do not instantaneously 

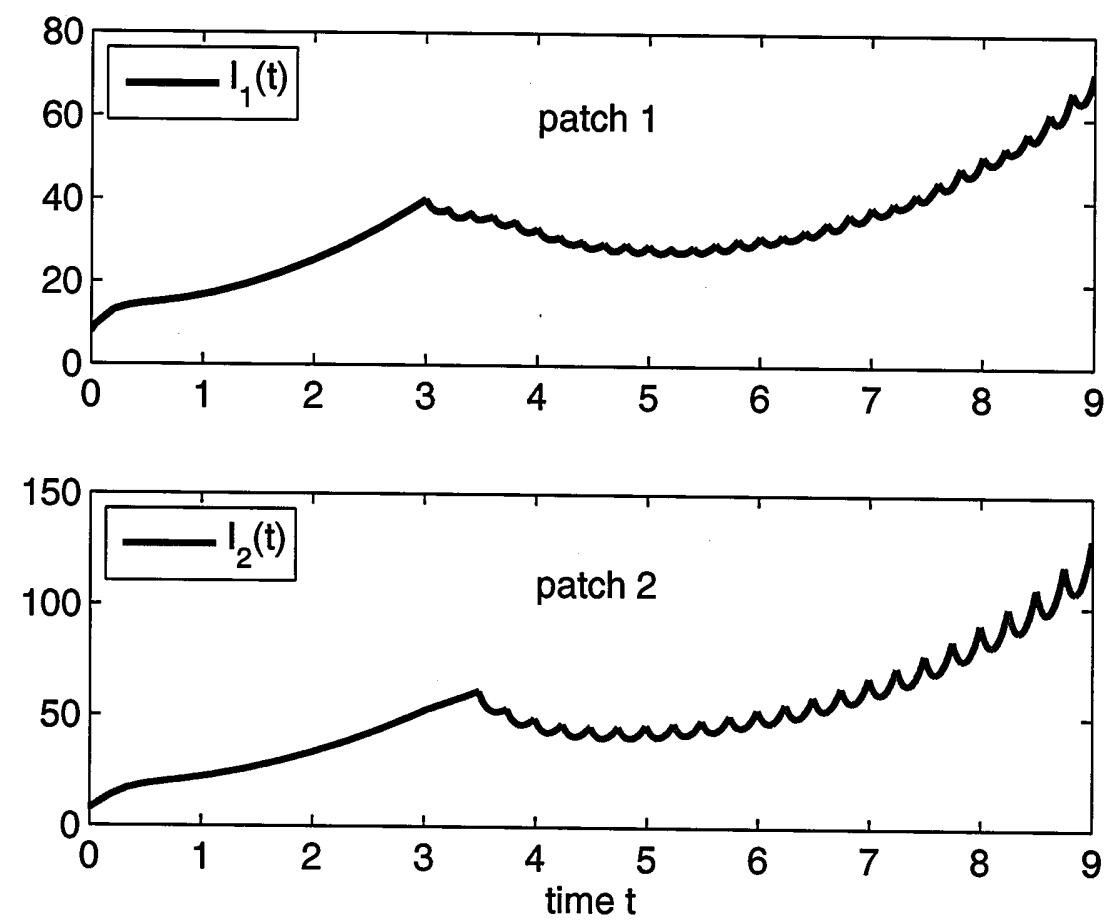

Figure 9.5: Independent pulse vaccination strategies on two patches, with pulses starting at time $t_{1}=3$ on patch 1 and time $t_{2}=3.5$ on patch 2 . The birth function is linear on both patches. Infective populations depicted. See the first two paragraphs of section 9.6 for details.

change the total population on patch $j$ or on any other patch.

In figure 9.6 we plot the number of infectives on patch 1 and 2 by simulating the model of (9.2), (9.3), and (9.4), with the same initial data, model parameters, and Nicholson-type birth functions as those used in figure 9.2. A PVS with constant pulse strength $p_{1}=0.97$ (so that $q_{1}=1-p_{1}=0.03$ ) begins on patch 1 at time $t_{1}=2$, with pulses occurring thereafter every $T_{1}$ time units where $T_{1}$ is found by setting equality in (9.51) for $j=1$, which gives $T_{1}=0.0035$. A PVS with constant pulse strength $p_{2}=0.95$ (so that $q_{2}=1-p_{2}=0.05$ ) begins on patch 2 at time $t_{2}=2.35$, with pulses occurring thereafter every $T_{2}$ time units where $T_{2}$ is found by setting equality in (9.51) for $j=2$, which gives $T_{2}=0.0021$. If we were to choose $T_{1}$ and $T_{2}$ to be anything less than the values obtained by setting equality in (9.51) for $j=1$ and 2 respectively, then theorem 9.3 would guarantee that $I_{1}(t) \rightarrow 0$ and $I_{2}(t) \rightarrow 0$ as $t \rightarrow \infty$. As it is, even by choosing $T_{1}$ and $T_{2}$ from setting equality in (9.51), figure 9.6 still apparently shows that $I_{1}(t) \rightarrow 0$ and $I_{2}(t) \rightarrow 0$. But this should not necessarily surprise us. We have not attempted to calculate the weakest pulse vaccination strategies that succeed in eradication - weakest in terms of frequency of application. Our theorems have been constructed to show simply that it is possible for independent pulse vaccination strategies to collectively wipe out the disease from all patches simultaneously. Future research could involve constructing the weakest successful independent pulse vaccination strategies. 

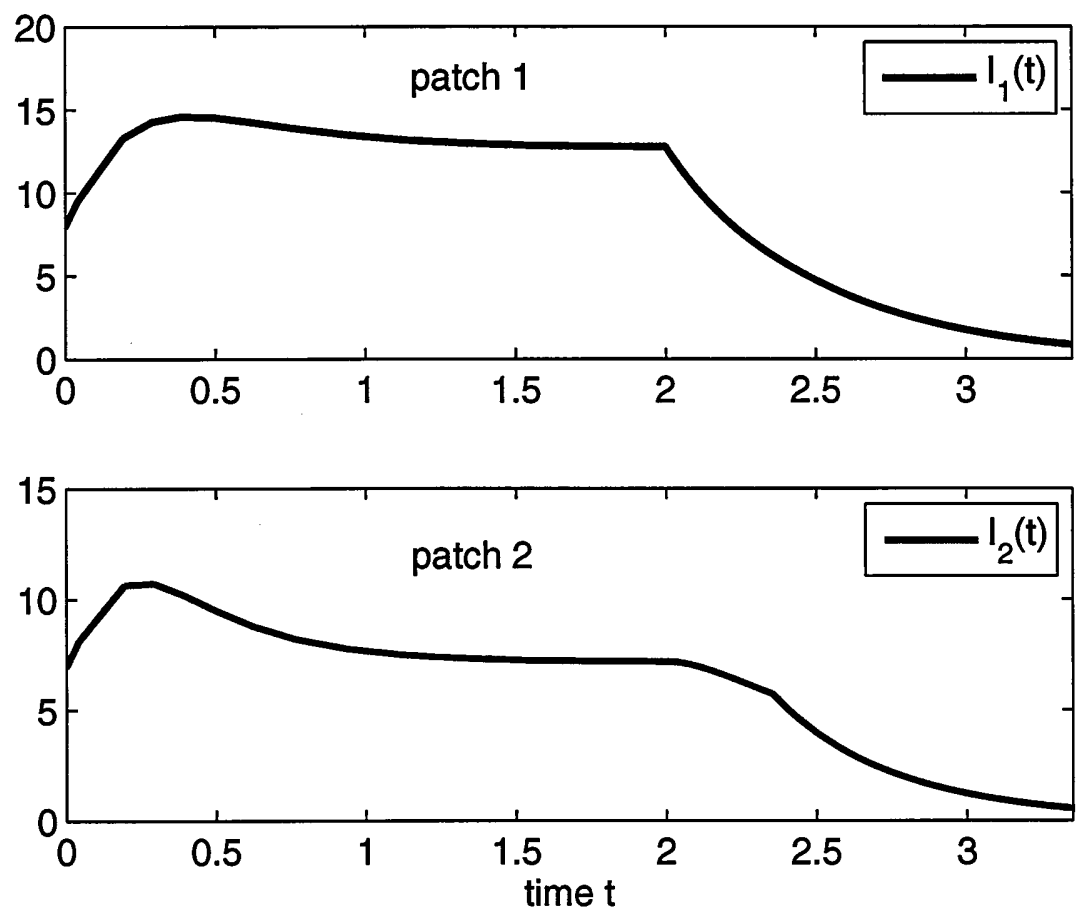

Figure 9.6: Successful independent pulse vaccination strategies on two patches, with pulses starting at time $t_{1}=2$ on patch 1 and time $t_{2}=2.35$ on patch 2 . The birth function is of Nicholson type on both patches. Infective populations depicted. Pulse frequencies are sufficiently high that it is difficult to discern the effect of individual pulses by eye. See the fourth paragraph of section 9.6 for details. 

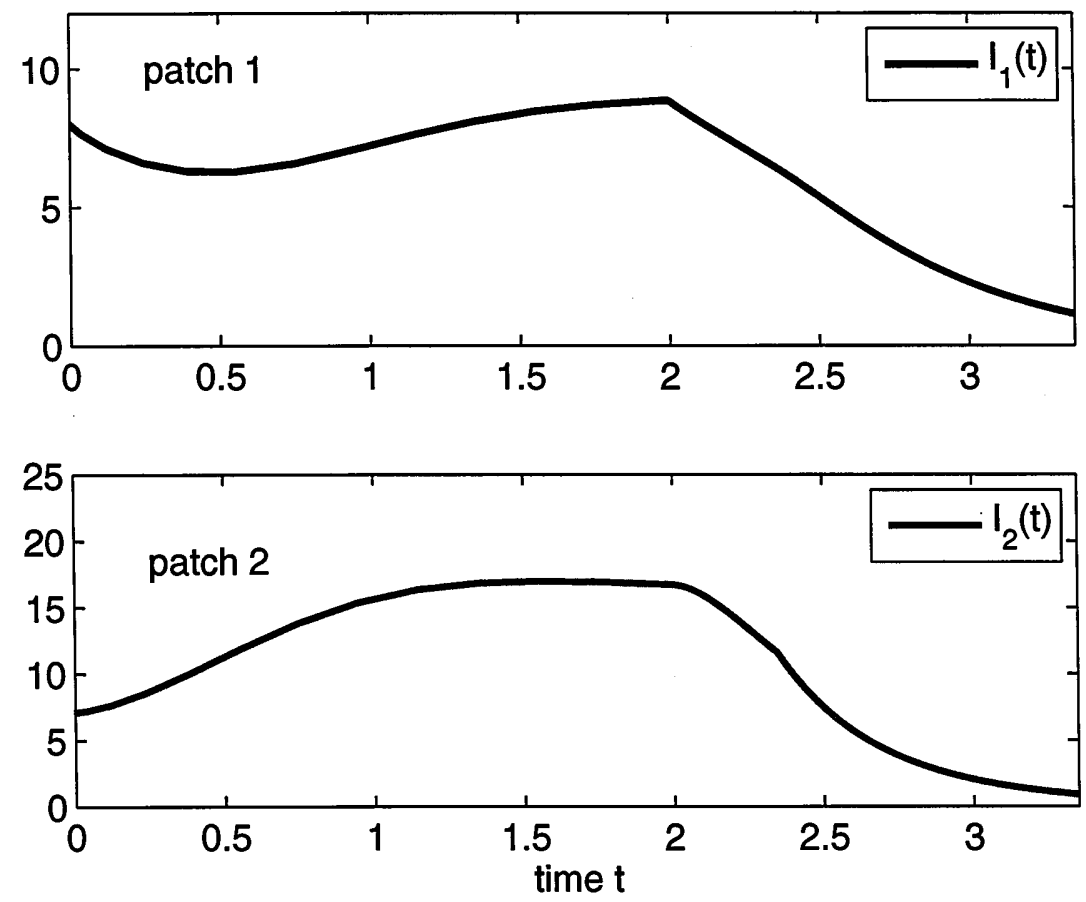

Figure 9.7: Successful independent pulse vaccination strategies on two patches, with pulses starting at time $t_{1}=2$ on patch 1 and time $t_{2}=2.35$ on patch 2 . The birth function is of Allee type on both patches. Infective populations depicted. Pulse frequencies are sufficiently high that it is difficult to discern the effect of individual pulses by eye. See the final paragraph of section 9.6 for details.

In figure 9.7 we plot the number of infectives on patch 1 and 2 by simulating the model of (9.2), (9.3), and (9.4), with the same initial data, model parameters, and Allee-type birth functions as those used in figure 9.3. A PVS with constant pulse strength $p_{1}=0.97$ (so that $q_{1}=1-p_{1}=0.03$ ) begins on patch 1 at time $t_{1}=2$, with pulses occurring thereafter every $T_{1}$ time units where $T_{1}$ is found by setting equality in (9.51) for $j=1$, which gives $T_{1}=0.0014$. A PVS with constant pulse strength $p_{2}=0.95$ (so that $q_{2}=1-p_{2}=0.05$ ) begins on patch 2 at time $t_{2}=2.35$, with pulses occurring thereafter every $T_{2}$ time units where $T_{2}$ is found by setting equality in (9.51) for $j=2$, which gives $T_{2}=0.0034$. The comments made about figure 9.6 apply equally well to figure 9.7 .

\subsection{Discussion}

We have recognised that inter-city, inter-regional, and international travel has increased significantly in the last few decades, promoting the spread of infectious diseases and motivating independent health authorities to co-ordinate their diseasecontrol initiatives. The spread of SARS (Severe Acute Respiratory Syndrome) across 
aviation routes in 2003 is a well-studied example [126]. Despite co-ordinated control efforts, independent health authorities are likely to retain at least some autonomy in their decisions. In particular, autonomy may be retained in choosing the precise details of a pulse vaccination strategy (PVS).

All of these considerations led us to examine an SIR model on $n \geq 2$ patches between any pair of which migration was permitted in either direction. Having seen by simulation that the disease can remain endemic on the different patches, we asked if it could be eradicated on all patches simultaneously if an independent PVS were carried out on each patch. We discovered that, if each PVS were sufficiently strong, then such eradication would occur. This result was corroborated by simulations in a two-patch model.

Future research could involve rigorously proving that the disease can remain endemic in the absence of vaccinations and constructing the weakest pulse vaccination strategies that still succeed - weakest in terms of frequency of pulses. 


\title{
Chapter 10
}

\section{Epidemiological models with a gestation delay}

\author{
"Defer no time, delays have dangerous ends." \\ William Shakespeare (1564 - 1616)
}

\subsection{Introduction}

The majority of epidemiological models in the literature, at least those intended for mammalian species, make no reference at all to the gestation period of the host. This may not matter when the duration of an epidemic is short enough in comparison to the natural turnover of the population that births and natural deaths may effectively be ignored, but when a disease is endemic there is no reason a priori to ignore gestation. In this chapter we therefore derive and examine an SIR model with a gestation delay in the birth term.

It is clear enough that diseases can affect conception and gestation. For example, rubella can cause miscarriages or cause a child to be born with congenital rubella syndrome [74,124]. In teenage males and men, mumps can cause infertility [122]. Fatal diseases, such as HIV/AIDS, passed on by vertical transmission (mother to unborn child in the womb) will obviously also affect conception rates, particularly if an infected child dies before reaching sexual maturity. A fatal disease will clearly affect gestation if it kills a pregnant woman.

So diseases can influence gestation but now we ask, can the gestation period influence the spread of a disease? Certainly the gestation period will affect the birth rate. Assuming for simplicity that the gestation period is a fixed constant $\tau>0$, then the number of new borns at a time $t$ will be wholly dependent on the number of conceptions at time $t-\tau$. But if all new borns are susceptible and recovery confers permanent immunity (as it may do with viral infections such as mumps, measles, or rubella [98]), and assuming the population is closed (no migration into or out of it), then the birth rate alone will be what feeds the disease with a fresh stock of victims and gives it the 
chance to persist. Intuitively, then, we can link endemicity to the gestation period. Thus there would appear to be value in incorporating gestation into epidemiological models. We will do this for an SIR model.

The model that we study will primarily be intended for a population, such as human, which breeds year round and where unborn members are protected from the disease by their mother's womb. However, our model may also be appropriate for a disease spreading through the adult stage of a creature with a life cycle of two stages where $\tau$ represents the maturation age. Creatures with stage-structured life cycles include crop pests which can spread diseases to the crops they infest. Even if the disease is non-fatal to the pest, it may yet damage the crop. In such a situation it would be in our interests to know if the disease can persist in the pest population. Similarly, it is in our interests to know when fatal diseases reduce populations of creatures we rely on, including pollinators such as bees.

The format of this chapter is as follows. In section 10.2 we derive an SIR model with a gestation delay when the disease can be fatal. In section 10.3 we show how the model simplifies when the disease is assumed to be non-fatal. In section 10.4 we show that there are circumstances under which the non-fatal disease of section 10.3 can be endemic. This prompts us to construct, in section 10.5, constant and pulse vaccination strategies that successfully control the disease. Simulations corroborate our results in section 10.6. In section 10.7 we derive, simulate, and comment on two insect pathogen models. We end the chapter with a discussion in section 10.8.

\subsection{An SIR model}

Suppose an infectious disease is circulating in a mammalian population in which unborn members are protected from the disease by their mother's womb - there is no vertical transmission. However, unborn members may die as a result of the disease if the mother dies from it. The gestation period is understood to mean the duration of pregnancy, the time from conception to birth. The gestation period will be treated for simplicity as a constant $\tau>0$. Examples of typical mammalian gestation periods are provided in table 10.1.

It is worth noting that almost 100 mammalian species are known to practise delayed implantation (or embryonic diapause) in which the blastocyst (early embryo) can be maintained in a state of dormancy (diapause) for many months prior to implantation in the lining of the uterus or womb [119]. Such an adaptation has evolved to allow these species to give birth at a time most favourable to the survival of mother and offspring. An example is the walrus for which the diapause lasts for 3 to 4 months. The time from embryonic implantation to birth in a walrus is about 12 months, giving a typical gestation (conception to birth) of 15-16 months, as we have stated in table 10.1.

In some species the diapause can vary significantly. For instance, in the brown bear it ranges from 4 to 7 months to give the bear flexibility in choosing when to begin its hibernation whilst ensuring the cubs will be born in the safety of a winter's den. The total gestation for a brown bear is anything from 6 to 9 months. Since this is not constant (contrary to the assumption we have made for the purposes of constructing a mathematical model), we have chosen to omit this species from table 10.1. We have also deliberately omitted marsupials because we will assume, for our model, that once a creature has been born it is able to interact with other members of the population and not just its mother. After being born, a marsupial is protected by its 


\begin{tabular}{|l|l||l|l|}
\hline Mammal & Typical gestation & Mammal & Typical gestation \\
\hline Elephant & 22 months & Baboon & 6 months \\
White Rhinoceros & $16-18$ months & Domestic sheep & 5 months \\
Walrus & $15-16$ months & Capybara & $4-5$ months \\
Giraffe & $14-15$ months & Wild boar & $110-115$ days \\
Zebra & $12-13$ months & Tiger & $105-115$ days \\
Northern fur seal & 12 months & Beaver & 100 days \\
Bottlenose dolphin & 12 months & Leopard & $90-95$ days \\
Cape Buffalo & $11-12$ months & Cape hunting dog & $70-72$ days \\
Llama & $11-12$ months & Domestic cat & 65 days \\
Blue whale & $10-12$ months & Coyote & 63 days \\
Horse & 11 months & Gray wolf & $60-63$ days \\
American Bison & $9-10$ months & Red fox & $51-53$ days \\
Eland & 9 months & Hare & $40-50$ days \\
Human & 9 months & European hedgehog & 35 days \\
Hippopotamus & 8 months & Rabbit & $28-31$ days \\
Caribou & 8 months & Brown rat & 21 days \\
Chimpanzee & $7-8$ months & House mouse & $19-21$ days \\
Impala & 7 months & Syrian hamster & $16-18$ days \\
\hline
\end{tabular}

Table 10.1: Examples of mammalian gestation periods. Information on gestation periods may be found in reference [59].

mother's pouch but it may still be susceptible to diseases that its mother may carry or catch.

An individual will mean a member of the population that has already been born and does not include foetuses (this is a matter of nomenclature and not an ethical statement). Assume any particular individual must be either susceptible, infective, or removed (these terms are defined in section 7.2) with respect to the disease at any time $t$. Then the population is divided into susceptible, infective, and removed classes. Let there be $S(t)$ susceptibles, $I(t)$ infectives, and $R(t)$ removeds at time $t$. Let the total number of individuals at time $t$ be $N(t)$. Then $N(t)=S(t)+I(t)+R(t)$. Assume for simplicity that there is homogeneous mixing and that all individuals have the same average number of contacts $\beta>0$ per unit time where $\beta$ is a constant. The assumptions of the last sentence will lead to a standard incidence function (see subsection 8.1.1). Recovery is assumed to confer permanent immunity. We will assume that there may be disease-caused deaths in deriving a model and then we will consider the special case in which the disease is non-fatal when we analyse the model.

Define the following parameters:

- $\mu_{s}=$ per capita death rate for susceptibles and removeds

- $\mu_{i}=$ per capita death rate for infectives

- $\gamma=$ per capita recovery rate for infectives .

Define $u(t, a)$ to be the density of foetuses at time $t$ of age $a$ where $0 \leq a \leq \tau$. Then the number of foetuses from age $a$ to $a+d a$ at time $t$ is approximately $u(t, a) d a$, with accuracy as $d a \rightarrow 0$. Since animals age at the same rate at which time passes, it is also correct to say that the number of foetuses from age $a$ to $a+d t$ at time $t$ is 
approximately $u(t, a) d t$, with accuracy as $d t \rightarrow 0$. Note that $u(t, a)$ may be interpreted as the rate at which foetuses pass through age $a$ at time $t$.

Since the probability of a miscarriage is not, in general, independent of the age of the foetus (younger foetuses in humans, for example, are more likely to miscarry (p. 835, [120])), let the miscarriage rate for a foetus of age $a$ be a function of $a$, namely $\mu_{f}(a)$.

Let the reproducing adult population at time $t$ be $M(t)$. The reproducing adult population is a proportion $\alpha$ of the total "born" population $N(t)$. This proportion $\alpha$ may change with time but in some populations, such as human populations, it may change slowly, particularly in relation to the gestation period. Thus, in Britian, the population is ageing but it is not ageing extremely rapidly. Over certain timescales, then, we can think of $\alpha$ as being constant. But then, over such a time-scale, $M(t)=\alpha N(t)$. Let us assume that we are modelling a population over such a timescale.

Now it is clear that the conception rate will be a function of the reproducing adult population $M(t)$ as well as a function of $t$ itself because, in many species, breeding occurs only at certain times called mating seasons. But since we are assuming that $M(t)=\alpha N(t)$ where $\alpha$ is a constant, we can say that the conception rate is a function of the total population $N(t)$ as well as of $t$. Denote this function as $b(N(t), t)$. We will refer to this function as the conception function or conception rate.

Notice that we may interpret $u(t, 0)$ to be the rate at which foetuses pass through age zero, which amounts to saying that $u(t, 0)$ is also the conception rate. We may therefore write:

$$
\text { conception rate at time } t=b(N(t), t)=u(t, 0)
$$

Next observe that $u(t, \tau)$ is the rate at which foetuses pass through age $\tau$, which means that $u(t, \tau)$ is the birth rate since $\tau$ is the gestation period. Since we have assumed there is no vertical transmission, these $u(t, \tau)$ new borns must be susceptible.

Given all of these assumptions, a sensible model is:

$$
\begin{aligned}
\frac{d S(t)}{d t} & =u(t, \tau)-\beta \frac{S(t) I(t)}{N(t)}-\mu_{s} S(t) \\
\frac{d I(t)}{d t} & =\beta \frac{S(t) I(t)}{N(t)}-\left(\gamma+\mu_{i}\right) I(t) \\
\frac{d R(t)}{d t} & =\gamma I(t)-\mu_{s} R(t) .
\end{aligned}
$$

The task now is to find an expression for $u(t, \tau)$ in terms of known parameters and functions. To this end, consider the foetuses that are of age $a$ at time $t$. These foetuses have density $u(t, a)$. (A group of organisms of the same or similar age is called a cohort.) For these foetuses, the death rate per foetus due to miscarriage is $\mu_{f}(a)$. In addition, the death rate per foetus due to the death of its mother will be the death rate per mother. But the proportion of foetuses whose mother is not infective is $\frac{S(t)+R(t)}{N(t)}$, and these mothers have a death rate per individual of $\mu_{s}$. Also the proportion of foetuses whose mother is infective is $\frac{I(t)}{N(t)}$, and these mothers have a death rate per individual of $\mu_{i}$. Then, in a narrow time interval $[t, t+d t]$, the number of foetal deaths we would expect is:

$$
\mu_{f}(a) u(t, a) d t+\mu_{s} \frac{S(t)+R(t)}{N(t)} u(t, a) d t+\mu_{i} \frac{I(t)}{N(t)} u(t, a) d t
$$


with equality for this expression as $d t \rightarrow 0$. Hence the change in the number of foetuses in $[t, t+d t]$ is

$$
\begin{gathered}
u(t+d t, a+d t)-u(t, a) \approx-\mu_{f}(a) u(t, a) d t-\mu_{s}\left(\frac{S(t)+R(t)}{N(t)}\right) u(t, a) d t \\
-\mu_{i} \frac{I(t)}{N(t)} u(t, a) d t
\end{gathered}
$$

with equality as $d t \rightarrow 0$.

A Taylor series expansion gives

$$
u(t+d t, a+d t)-u(t, a)=\frac{\partial u(t, a)}{\partial t} d t+\frac{\partial u(t, a)}{\partial a} d t+O\left(d t^{2}\right),
$$

where $O\left(d t^{2}\right)$ represents terms containing $(d t)^{2}$ or higher powers of $d t$.

Equating the right hand sides of (10.6) and (10.7), dividing by $d t$, and letting $d t \rightarrow$ 0 , leads to:

$$
\frac{\partial u(t, a)}{\partial t}+\frac{\partial u(t, a)}{\partial a}=-\left(\mu_{f}(a)+\mu_{s} \frac{S(t)+R(t)}{N(t)}+\mu_{i} \frac{I(t)}{N(t)}\right) u(t, a) .
$$

Adopting an approach used by Simons and Gourley [125], we now define $u_{\xi}(a)=$ $u(a+\xi, a)$. Then, using (10.8), we can write:

$$
\begin{aligned}
\frac{d u_{\xi}}{d a} & =\left[\frac{\partial u(t, a)}{\partial t}+\frac{\partial u(t, a)}{\partial a}\right]_{t=a+\xi} \\
& =\left[-\left(\mu_{f}(a)+\mu_{s} \frac{S(t)+R(t)}{N(t)}+\mu_{i} \frac{I(t)}{N(t)}\right) u(t, a)\right]_{t=a+\xi} \\
& =\left(-\left(\mu_{f}(a)+\mu_{s} \frac{S(a+\xi)+R(a+\xi)}{N(a+\xi)}+\mu_{i} \frac{I(a+\xi)}{N(a+\xi)}\right)\right) u_{\xi}
\end{aligned}
$$

Solving for $u_{\xi}$ gives:

$$
u_{\xi}(a)=u_{\xi}(0) \exp \left(-\int_{0}^{a} \mu_{f}(\eta)+\mu_{s} \frac{S(\eta+\xi)+R(\eta+\xi)}{N(\eta+\xi)}+\mu_{i} \frac{I(\eta+\xi)}{N(\eta+\xi)} d \eta\right)
$$

If we now set $\xi=t-\tau$ and $a=\tau$, then (10.10) becomes:

$$
\begin{gathered}
u(t, \tau)=u(t-\tau, 0) \\
\times \exp \left(-\int_{0}^{\tau} \mu_{f}(\eta)+\mu_{s} \frac{S(\eta+t-\tau)+R(\eta+t-\tau)}{N(\eta+t-\tau)}+\mu_{i} \frac{I(\eta+t-\tau)}{N(\eta+t-\tau)} d \eta\right)
\end{gathered}
$$

But by $(10.1)$, we can say that $u(t-\tau, 0)=b(N(t-\tau), t-\tau)$. Combining this fact with (10.11) yields the required expression for the birth rate $u(t, \tau)$ :

$$
\begin{aligned}
& u(t, \tau)=b(N(t-\tau), t-\tau) e^{-\int_{0}^{\tau} \mu_{f}(\eta) d \eta} \\
& \quad \times \exp \left(-\int_{0}^{\tau} \mu_{s} \frac{S(\eta+t-\tau)+R(\eta+t-\tau)}{N(\eta+t-\tau)}+\mu_{i} \frac{I(\eta+t-\tau)}{N(\eta+t-\tau)} d \eta\right)
\end{aligned}
$$

The terms in (10.12) may be interpreted ecologically. The birth rate at time $t$ (namely $u(t, \tau))$ is equal to the conception rate at time $t-\tau$ (namely $b(N(t-\tau), t-\tau)$ ), multiplied 
by the probability that a foetus does not miscarry (namely $e^{-\int_{0}^{\tau} \mu_{f}(\eta) d \eta}$ ), multiplied by the probability that the mother does not die (which would kill the foetus).

For a properly defined model, our final requirement is initial data:

$$
S(t)=S_{0}(t) \geq 0, \quad I(t)=I_{0}(t) \geq 0, \quad R(t)=R_{0}(t) \geq 0 \quad \text { for } t \in[-\tau, 0] .
$$

Here $S_{0}, I_{0}$, and $R_{0}$ are initial functions or functions of initial data.

Our SIR model is now defined completely by (10.2), (10.3), (10.4), (10.12), and by (10.13). For this model, a unique solution exists for $t>0$ provided the conception function $b(\cdot)$ is suitably sensible. We will not enter into a discussion of the existence and uniqueness of solutions to delay differential systems here but instead observe that a proper discussion may be found in [95].

\subsection{A simple model}

In this section and the three sections which follow it, we explore a special case of the model derived in section 10.2. First suppose that breeding takes place year-round (as in a human population) so that the conception function depends only on the total population $N(t)$. Moreover, assume this dependence is linear, so that $b(N(t))=\lambda N(t)$ for some constant $\lambda>0$. Next suppose that the disease is non-fatal, in which case $\mu_{s}=\mu_{i}$. By setting $\mu=\mu_{s}=\mu_{i}$, then (10.12) simplifies:

$$
u(t, \tau)=\Lambda N(t-\tau),
$$

where $\Lambda=\lambda e^{-\int_{0}^{\tau} \mu_{f}(\eta) d \eta} e^{-\mu \tau}$.

Combining (10.14) with (10.2), (10.3), and (10.4), our model becomes:

$$
\begin{aligned}
\frac{d S(t)}{d t} & =\Lambda N(t-\tau)-\beta \frac{S(t) I(t)}{N(t)}-\mu S(t) \\
\frac{d I(t)}{d t} & =\beta \frac{S(t) I(t)}{N(t)}-(\gamma+\mu) I(t) \\
\frac{d R(t)}{d t} & =\gamma I(t)-\mu R(t) .
\end{aligned}
$$

This model is still subject to the initial data in (10.13), which we repeat here for ease of reference:

$$
S(t)=S_{0}(t) \geq 0, \quad I(t)=I_{0}(t) \geq 0, \quad R(t)=R_{0}(t) \geq 0 \quad \text { for } t \in[-\tau, 0] .
$$

We assume additionally that:

$$
S(0)>0 \text { and } I(0)>0 .
$$

We will find conditions for the disease to persist in the population in section 10.4. Since the model could apply to a disease for a human population, and humans prefer to eradicate their own diseases where possible, we construct successful vaccination strategies in section 10.5. Note that even a non-fatal disease can adversely affect a country's economy by causing children to miss days at school and adults to miss days at work.

Recall the description of a constant vaccination strategy (CVS) and pulse vaccination strategy (PVS) from section 7.4. If we incorporate a CVS into the model of (10.15), 
(10.16), and (10.17), where a constant proportion $\pi$ of all new borns is vaccinated successfully and on a continuous basis, then the model becomes:

$$
\begin{aligned}
\frac{d S(t)}{d t} & =(1-\pi) \Lambda N(t-\tau)-\beta \frac{S(t) I(t)}{N(t)}-\mu S(t) \\
\frac{d I(t)}{d t} & =\beta \frac{S(t) I(t)}{N(t)}-(\gamma+\mu) I(t) \\
\frac{d R(t)}{d t} & =\gamma I(t)-\mu R(t)+\pi \Lambda N(t-\tau) .
\end{aligned}
$$

Since $\pi$ is a proportion, then $0 \leq \pi \leq 1$. If $\pi=0$, then no new borns are vaccinated successfully and the model reduces to the model with no vaccinations. If $\pi=1$, then all new borns are vaccinated successfully, and it becomes trivial to show that the disease must inevitably die out. Assume for the rest of this chapter, then, that $0 \leq \pi<1$.

In the case where the disease is non-fatal, we may incorporate a PVS into the model without fear of creating inconsistencies in the model derivation of section 10.2. This is because, for a non-fatal disease, the process of deriving the birth rate $u(t, \tau)$ will not involve dependence on the sizes of the individual disease classes (susceptible, infective, removed). The correct process of deriving $u(t, \tau)$ when there is a PVS and the disease can be fatal is a matter for future research.

Incorporating a PVS into the model for a non-fatal disease, as outlined in definition 8.1 , we see that the system evolves between pulses in accordance with equations (10.15), (10.16), and (10.17), but at each pulse the numbers in each class are reset thus:

$$
S\left(t_{i}\right)=(1-p) S\left(t_{i}^{-}\right), \quad I\left(t_{i}\right)=I\left(t_{i}^{-}\right), \quad R\left(t_{i}\right)=R\left(t_{i}^{-}\right)+p S\left(t_{i}^{-}\right) .
$$

The model with a PVS therefore consists of a series of initial value problems, with a new problem beginning with each pulse. Assume that the pulses do not attain complete coverage (which would anyway be unrealistic for a real-world program), that is, assume $0 \leq p<1$. Let $q=1-p$.

For the three models stated (no vaccinations, CVS, PVS), subject to the initial data in (10.18) and (10.19), unique solutions exist for $t>0$ (see [95] for results on the existence and uniqueness of solutions). Using the non-negativity of the initial data (10.18), it is a routine matter (see p. 81, [128]) to show that the three models (no vaccinations, CVS, PVS) satisfy positivity, that is, for $t \geq 0$, we have $S(t) \geq 0, I(t) \geq 0$, and $R(t) \geq 0$. Since (10.19) is also assumed to hold, positivity can be shown to be strict, that is, $S(t)>0, I(t)>0$, and $R(t)>0$ (and therefore $N(t)>0$ ) for $t \geq 0$.

Notice that the model with no vaccinations (equations (10.15), (10.16), and (10.17)) may not only be appropriate to mammalian populations. It may also be appropriate for a reptilian or amphibian population in which foetuses are protected from vertical transmission by encasement in an egg rather than within a uterus. However, the model may not be appropriate for all types of egg-laying creatures - bird hatchlings do not tend to mix homogeneously, being restricted to a nest.

\subsection{Model analysis}

The principle value of mathematical epidemiology is arguably in the control of diseases. Now a disease may not need to be controlled if it does not persist in a popula- 
tion. In this section we ask when the disease modelled by (10.15), (10.16), and (10.17) dies out and when it can be endemic.

If we add together (10.15), (10.16), and (10.17), and bearing in mind that $N(t)=$ $S(t)+I(t)+R(t)$, we obtain:

$$
\frac{d N(t)}{d t}=\Lambda N(t-\tau)-\mu N(t) \text { for } t>0 .
$$

Equation (10.24) also holds for the model with a CVS (as we can see by adding together equations (10.20), (10.21), and (10.22)) and for the model with a PVS (because the model equations are the same between pulses as in the model with no vaccinations and each instantaneous pulse does not alter the total size of the population).

By (10.24) and the results of subsection 3.3.1 we see that the evolution of the population $N(t)$ will depend upon the size of $\frac{\Lambda}{\mu}$. Suppose that

$$
M \leq N(t) \leq W \text { for } t \in[-\tau, 0],
$$

where $M$ and $W$ are positive constants. If $\frac{\Lambda}{\mu}<1$ then by theorem 3.1 we find that $N(t) \rightarrow 0$ as $t \rightarrow \infty$ in such a way that $N(t)$ can be bounded above by a decreasing sequence. If $\frac{\Lambda}{\mu}=1$ then by theorem 3.2 the population is "stable" in the sense that $M \leq N(t) \leq W$ for all $t>0$. Finally if $\frac{\Lambda}{\mu}>1$ then by theorem 3.3 we find that $N(t) \rightarrow \infty$ as $t \rightarrow \infty$ in such a way that $N(t)$ can be bounded below by an increasing sequence.

In the following lemma, we see that the disease will die out if the contact rate $\beta$ is small enough.

Lemma 10.1. In the model of (10.15), (10.16), (10.17), (10.18), and (10.19), suppose that $\beta<\mu+\gamma$. Then $I(t) \rightarrow 0$ as $t \rightarrow \infty$.

Proof. Let $\alpha=\mu+\gamma-\beta$. Since $\beta<\mu+\gamma$ by assumption, then $\alpha>0$. By positivity we know that $\frac{S(t)}{N(t)} \leq 1$ for $t \geq 0$. But then, by (10.16), we have $\frac{d I(t)}{d t} \leq-\alpha I(t)$ for $t \geq 0$. Hence for $t \geq 0$, we can say that $I(t) \leq I^{*}(t)$ where $I^{*}(0)=I(0)>0$ and where, for $t \geq 0, \frac{d I^{*}(t)}{d t}=-\alpha I^{*}(t)$. Solving for $I^{*}(t)$ and using the positivity of $I(t)$, we can therefore write:

$$
0 \leq I(t) \leq I^{*}(t)=I(0) e^{-\alpha t} \text { for } t \geq 0 .
$$

Since $\alpha>0$, we deduce by (10.26) that $I(t) \rightarrow 0$ as $t \rightarrow \infty$.

Next we show that the disease can remain relatively endemic (the proportion of infectives is bounded below by a positive constant) given certain conditions on the model parameters and initial data. To do this, we must first prove a lemma:

Lemma 10.2. Suppose that (10.24) and (10.25) hold. Then:

$$
A\left(\frac{M}{W}\right) \leq \frac{N(t-\tau)}{N(t)} \leq B\left(\frac{W}{M}\right) \text { for } t \geq 0,
$$

where $A=\min \left\{1, \frac{\mu}{\Lambda}\right\}$ and $B=\max \left\{1, \frac{\mu}{\Lambda}\right\}$. 
Proof. The result is clearly true if we can prove that (10.27) holds for $t \in[j \tau,(j+1) \tau]$ by induction on $j \geq 0$.

\section{Basis step $(j=0)$}

We need to show that (10.27) holds for $t \in[0, \tau]$. To this end, first note that, since $N(t) \geq M$ for $t \in[-\tau, 0]$ by (10.25), then by (10.24) we have:

$$
\frac{d N(t)}{d t} \geq \Lambda M-\mu N(t) \text { for } t \in[0, \tau] \text {. }
$$

It follows that $N(t) \geq \bar{N}(t)$ for $t \in[0, \tau]$ where $\bar{N}(0)=M \leq N(0)$ and $\frac{d \bar{N}(t)}{d t}=$ $\Lambda M-\mu \bar{N}(t)$ for $t \in[0, \tau]$. Solving for $\bar{N}(t)$ reveals that:

$$
N(t) \geq \bar{N}(t)=\frac{\Lambda}{\mu} M+M\left(1-\frac{\Lambda}{\mu}\right) e^{-\mu t} \text { for } t \in[0, \tau] .
$$

Similarly, since $N(t) \leq W$ for $t \in[-\tau, 0]$ we find that:

$$
N(t) \leq \frac{\Lambda}{\mu} W+W\left(1-\frac{\Lambda}{\mu}\right) e^{-\mu t} \text { for } t \in[0, \tau]
$$

If $\Lambda \leq \mu$ then the lower bound in (10.29) is a monotonic non-increasing function of $t$, and so is the upper bound in (10.30). Hence:

$$
\frac{\Lambda}{\mu} M \leq \frac{\Lambda}{\mu} M+M\left(1-\frac{\Lambda}{\mu}\right) e^{-\mu \tau} \leq N(t) \leq W \text { for } t \in[0, \tau] .
$$

Combining (10.25) with (10.31) and the assumption that $\Lambda \leq \mu$ yields:

$$
A\left(\frac{M}{W}\right)=\frac{M}{W} \leq \frac{N(t-\tau)}{N(t)} \leq\left(\frac{\mu}{\Lambda}\right)\left(\frac{W}{M}\right)=B\left(\frac{W}{M}\right) \text { for } t \in[0, \tau] .
$$

If $\Lambda>\mu$ then the lower bound in (10.29) is a monotonic increasing function of $t$, and so is the upper bound in (10.30). Hence:

$$
M \leq N(t) \leq\left(\frac{\Lambda}{\mu}\right) W \text { for } t \in[0, \tau]
$$

Combining (10.25) with (10.33) and the assumption that $\Lambda>\mu$ yields:

$$
A\left(\frac{M}{W}\right)=\left(\frac{\mu}{\Lambda}\right)\left(\frac{M}{W}\right) \leq \frac{N(t-\tau)}{N(t)} \leq \frac{W}{M}=B\left(\frac{W}{M}\right) \text { for } t \in[0, \tau] .
$$

By (10.32) and (10.34) we see that (10.27) holds for $t \in[0, \tau]$, as required.

\section{Inductive step}

Assume (inductive hypothesis) that (10.27) holds for $t \in[j \tau,(j+1) \tau]$ for some $j \geq 0$. Then we want to show that $(10.27)$ holds for $t \in[(j+1) \tau,(j+2) \tau]$. To that end, note that:

$$
\frac{d}{d t}\left(\frac{N(t-\tau)}{N(t)}\right)=\frac{N(t) \frac{d N(t-\tau)}{d t}-N(t-\tau) \frac{d N(t)}{d t}}{(N(t))^{2}}
$$


But:

$$
\begin{aligned}
\frac{d N(t-\tau)}{d t} & =\frac{d N(t-\tau)}{d(t-\tau)} \frac{d(t-\tau)}{d t}=\frac{d N(t-\tau)}{d(t-\tau)} \\
& =\Lambda N(t-2 \tau)-\mu N(t-\tau) \text { using (10.24). }
\end{aligned}
$$

Hence, letting $z(t)=\frac{N(t-\tau)}{N(t)}$, we find by (10.35), (10.36), and (10.24) that:

$$
\frac{d z(t)}{d t}=\Lambda z(t)(z(t-\tau)-z(t)) \text { for } t>0
$$

Now $N(t)$ satisfies strict positivity for $t \geq-\tau$ (see proposition 2.1, [125]). But then clearly $z(t)$ satisfies strict positivity for $t \geq 0$. Then, from the inductive hypothesis and (10.37) we find that:

$\Lambda z(t)\left(A\left(\frac{M}{W}\right)-z(t)\right) \leq \frac{d z(t)}{d t} \leq \Lambda z(t)\left(B\left(\frac{W}{M}\right)-z(t)\right)$ for $t \in[(j+1) \tau,(j+2) \tau]$.

It follows that $z_{1}(t) \leq z(t) \leq z_{2}(t)$ for $t \in[(j+1) \tau,(j+2) \tau]$ where:

$$
z_{1}((j+1) \tau)=A\left(\frac{M}{W}\right) \leq z((j+1) \tau) \text { using inductive hypothesis }
$$

and

$$
\frac{d z_{1}(t)}{d t}=\Lambda z_{1}(t)\left(A\left(\frac{M}{W}\right)-z_{1}(t)\right) \text { for } t \in[(j+1) \tau,(j+2) \tau]
$$

and where

$$
z_{2}((j+1) \tau)=B\left(\frac{W}{M}\right) \geq z((j+1) \tau) \text { using inductive hypothesis }
$$

and

$$
\frac{d z_{2}(t)}{d t}=\Lambda z_{2}(t)\left(B\left(\frac{W}{M}\right)-z_{2}(t)\right) \text { for } t \in[(j+1) \tau,(j+2) \tau]
$$

Since $A\left(\frac{M}{W}\right)$ is a fixed point for the differential equation for $z_{1}(t)$ in (10.40), we immediately find by (10.39) and (10.40) that $z_{1}(t) \equiv A\left(\frac{M}{W}\right)$ for $t \in[(j+1) \tau,(j+2) \tau]$. By similar reasoning, we find by (10.41) and (10.42) that $z_{2}(t) \equiv B\left(\frac{W}{M}\right)$ for $t \in[(j+$ 1) $\tau,(j+2) \tau]$. Hence:

$$
A\left(\frac{M}{W}\right)=z_{1}(t) \leq z(t) \leq z_{2}(t)=B\left(\frac{W}{M}\right) \text { for } t \in[(j+1) \tau,(j+2) \tau] .
$$

Thus we have shown that (10.27) holds for $t \in[(j+1) \tau,(j+2) \tau]$, as required on the inductive step.

Theorem 10.1 (Endemicity). In the model of (10.15), (10.16), (10.17), (10.18), and (10.19), suppose that the initial population satisfies (10.25). Also assume that:

$$
\beta>\frac{4\left(\Lambda A\left(\frac{M}{W}\right)+\gamma\right)\left(\Lambda B\left(\frac{W}{M}\right)+\gamma\right)}{\Lambda A\left(\frac{M}{W}\right)},
$$


where $M$ and $W$ are as defined in (10.25) and $A$ and $B$ are defined in lemma 10.2. Define the constants $R_{1}, R_{2}, R_{3}$ as follows:

$$
\begin{aligned}
& R_{1}=\left(\frac{\beta}{4}\right)\left(\frac{\Lambda A\left(\frac{M}{W}\right)}{\Lambda A\left(\frac{M}{W}\right)+\gamma}\right)-\gamma-\Lambda B\left(\frac{W}{M}\right) \\
& R_{2}=\Lambda B\left(\frac{W}{M}\right) \\
& R_{3}=\frac{\beta}{2} .
\end{aligned}
$$

Clearly $R_{2}>0$ and $R_{3}>0$. Given (10.44) it is also clear that $R_{1}>0$. Then $P>0$ where:

$$
P=\frac{\sqrt{\left(R_{2}^{2}+4 R_{1} R_{3}\right)}-R_{2}}{2 R_{3}} .
$$

Also, there exists $\bar{t} \geq 0$ such that, for $t \geq \bar{t}$, we have:

$$
\frac{I(t)}{N(t)} \geq\left(\frac{1}{4}\right)\left(\frac{\Lambda A\left(\frac{M}{W}\right)}{\Lambda A\left(\frac{M}{W}\right)+\gamma}\right)+\frac{P}{4}
$$

Thus the disease is relatively endemic if condition (10.44) holds. Moreover the disease is absolutely endemic if $\Lambda \geq \mu$, for if $\Lambda=\mu$ then, for $t \geq \bar{t}$,

$$
I(t) \geq\left(\frac{1}{4 W}\right)\left(\frac{\Lambda A\left(\frac{M}{W}\right)}{\Lambda A\left(\frac{M}{W}\right)+\gamma}\right)+\frac{P}{4 W},
$$

and if $\Lambda>\mu$ then $I(t) \rightarrow \infty$ as $t \rightarrow \infty$.

Proof. Let $x=x(t)=\frac{S(t)}{N(t)}, \dot{x}=\frac{d x(t)}{d t}, y=y(t)=\frac{I(t)}{N(t)}$, and $\dot{y}=\frac{d y(t)}{d t}$. Our method will be to bound below $y+x$ for suitably large $t$, then to bound below $y-x$ for suitably large $t$, and then to add these lower bounds together to find a lower bound for the proportion of infectives $y(t)$ for time $t$ suitably large.

Now when (10.15), (10.16), and (10.17) hold, then (10.24) holds. Then, using (10.15), (10.16), (10.24), and the quotient rule for differentiation we find, for $t>0$, that:

$$
\begin{gathered}
\dot{x}=\Lambda \frac{N(t-\tau)}{N(t)}-\beta x y-\Lambda x \frac{N(t-\tau)}{N(t)}, \\
\dot{y}=y\left(\beta x-\gamma-\Lambda \frac{N(t-\tau)}{N(t)}\right) .
\end{gathered}
$$

Notice that, since $S(t), I(t)$, and $N(t)$ satisfy strict positivity for $t \geq-\tau$, then so do $x(t)$ and $y(t)$.

Add together (10.51) and (10.52) to obtain, for $t>0$,

$$
\dot{y}+\dot{x}=\Lambda \frac{N(t-\tau)}{N(t)}(1-(y+x))-\gamma y \text {. }
$$

By positivity we know that $0 \geq-\gamma x$ and that $1-(y+x) \geq 0$. Also, by lemma 10.2, we know that $\frac{N(t-\tau)}{N(t)} \geq A\left(\frac{M}{W}\right)$ for $t>0$. Combining these facts with (10.53) reveals that, for $t>0$,

$$
\dot{y}+\dot{x} \geq \Lambda A\left(\frac{M}{W}\right)(1-(y+x))-\gamma(y+x) .
$$


Let $z_{1}=z_{1}(t)=y(t)+x(t)=y+x$. Then by (10.54), we can write, for $t>0$,

$$
\dot{z}_{1} \geq \Lambda A\left(\frac{M}{W}\right)-\left(\Lambda A\left(\frac{M}{W}\right)+\gamma\right) z_{1} .
$$

It follows (theorem 1.1, pp. 78-79, [128]) that $z_{1}(t) \geq Z_{1}(t)=Z_{1}$ for $t \geq 0$ where $Z_{1}(0)=z_{1}(0)=\frac{S(0)}{N(0)}+\frac{I(0)}{N(0)}>0$ and where, for $t>0$,

$$
\frac{d Z_{1}}{d t}=\Lambda A\left(\frac{M}{W}\right)-\left(\Lambda A\left(\frac{M}{W}\right)+\gamma\right) Z_{1} .
$$

Trivially solving for $Z_{1}(t)$, we deduce that

$$
Z_{1}(t) \rightarrow \frac{\Lambda A\left(\frac{M}{W}\right)}{\Lambda A\left(\frac{M}{W}\right)+\gamma} \text { as } t \rightarrow \infty
$$

Then there exists $t^{*} \geq 0$ such that, for $t \geq t^{*}$, we have:

$$
y(t)+x(t)=z_{1}(t) \geq Z_{1}(t) \geq\left(\frac{1}{2}\right)\left(\frac{\Lambda A\left(\frac{M}{W}\right)}{\Lambda A\left(\frac{M}{W}\right)+\gamma}\right) .
$$

Now subtract (10.51) from (10.52) to obtain, for $t>0$,

$$
\dot{y}-\dot{x}=2 \beta x y-\gamma y-\Lambda \frac{N(t-\tau)}{N(t)}(1+(y-x)) .
$$

By positivity we know that $y \leq 1$, so that $-\gamma y \geq-\gamma$. Also by positivity $1+(y-x) \geq$ 0 , and by lemma 10.2 we know that $\frac{N(t-\tau)}{N(t)} \leq B\left(\frac{W}{M}\right)$ for $t>0$, so we must have $-\frac{N(t-\tau)}{N(t)}(1+(y-x)) \geq-B\left(\frac{W}{M}\right)(1+(y-x))$ for $t>0$. Notice furthermore that:

$$
\begin{aligned}
4 x y & =(y+x)^{2}-(y-x)^{2} \\
& \geq\left(\frac{1}{2}\right)\left(\frac{\Lambda A\left(\frac{M}{W}\right)}{\Lambda A\left(\frac{M}{W}\right)+\gamma}\right)-(y-x)^{2} \text { for } t \geq t^{*} \text { (using (10.57)) }
\end{aligned}
$$

Combining (10.60) and the inequalities stated immediately before it with (10.59) reveals that, for $t \geq t^{*}$,

$$
\dot{y}-\dot{x} \geq\left(\frac{\beta}{4}\right)\left(\frac{\Lambda A\left(\frac{M}{W}\right)}{\Lambda A\left(\frac{M}{W}\right)+\gamma}\right)-\left(\frac{\beta}{2}\right)(y-x)^{2}-\Lambda B\left(\frac{W}{M}\right)(1+(y-x)) .
$$

$$
\text { Let } z_{2}=z_{2}(t)=y(t)-x(t)=y-x \text {. Then by (10.61), we can write, for } t \geq t^{*} \text {, }
$$

$$
\dot{z}_{2} \geq R_{1}-R_{2} z_{2}-R_{3} z_{2}^{2}
$$

where $R_{1}, R_{2}$, and $R_{3}$ are defined in (10.45), (10.46), and (10.47) respectively. It

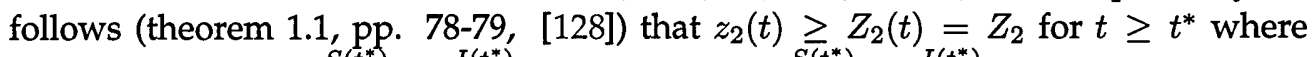
$Z_{2}\left(t^{*}\right)=z_{2}\left(t^{*}\right)=\frac{S\left(t^{*}\right)}{N\left(t^{*}\right)}+\frac{I\left(t^{*}\right)}{N\left(t^{*}\right)}>0$ (note that $\frac{S\left(t^{*}\right)}{N\left(t^{*}\right)}+\frac{I\left(t^{*}\right)}{N\left(t^{*}\right)}$ is positive by strict positivity) and where, for $t>t^{*}$,

$$
\frac{d Z_{2}}{d t}=R_{1}-R_{2} Z_{2}-R_{3} Z_{2}^{2} .
$$


By (10.44), it is easily seen that $R_{1}>0$. It is also obvious that $R_{2}>0$ and $R_{3}>0$. It follows that (10.63) has two fixed points, where one is negative and the other is positive. The positive fixed point is:

$$
P=\frac{\sqrt{R_{2}^{2}+4 R_{1} R_{3}}-R_{2}}{2 R_{3}} .
$$

Since $Z_{2}\left(t^{*}\right)>0$, a simple phase portrait analysis allows us to conclude that $Z_{2}(t) \rightarrow P$ as $t \rightarrow \infty$. But for $t \geq t^{*}, Z_{2}(t)$ is a lower bound for $z_{2}(t)=y(t)-x(t)$, which cannot exceed 1 for otherwise positivity would be contradicted. It is sensible to check, then, that $P \leq 1$. But by the definitions of $R_{1}, R_{2}$, and $R_{3}$ in (10.45), (10.46), and (10.47) and the knowledge that these three constants are positive, it is readily seen that $P<1$.

Now $Z_{2}(t)$ is defined for $t \geq t^{*}$ and we have said that $Z_{2}(t) \rightarrow P$ as $t \rightarrow \infty$. Then there exists $\bar{t} \geq t^{*}$ such that, for $t \geq \bar{t}$, we have

$$
y(t)-x(t)=z_{2}(t) \geq Z_{2}(t) \geq \frac{P}{2} .
$$

Adding (10.65) and (10.58) reveals that, for $t \geq \bar{t} \geq t^{*} \geq 0$,

$$
2 y(t) \geq\left(\frac{1}{2}\right)\left(\frac{\Lambda A\left(\frac{M}{W}\right)}{\Lambda A\left(\frac{M}{W}\right)+\gamma}\right)+\frac{P}{2} .
$$

Inequality (10.49) follows directly from (10.66).

Finally, the assertion that (10.50) holds for $t \geq \bar{t}$ follows trivially from (10.49) and the fact that $N(t) \leq W$ for $t \geq 0$ when $\Lambda=\mu$ (using theorem 3.2), and the assertion that $I(t) \rightarrow \infty$ as $t \rightarrow \infty$ when $\Lambda>\mu$ follows from (10.49) and the fact that $N(t) \rightarrow \infty$ as $t \rightarrow \infty$ when $\Lambda>\mu$ (using theorem 3.3).

Theorem 10.1 effectively says that if the per capita contact rate $\beta$ is sufficiently large compared to the per capita conception rate $\Lambda$ and per infective recovery rate $\gamma$ then the disease will persist as a proportion of the population. Such a result seems sensible enough given our assumption that a contact is adequate for disease transmission if it is between an infective and a susceptible. An analogous result is known to hold when there is no delay. In fact if we set $\tau=0$ in the model of (10.15), (10.16), (10.17), (10.18), and (10.19), then it is known by theorem 2.1 in [60] that the disease will be relatively endemic when $\beta>\Lambda+\gamma$. (Note that when $\tau=0$ then $\Lambda$ becomes the per capita birth rate instead of the per capita conception rate.) It would be of interest to know if the results which hold when there is no delay [60] carry over into the case when a gestation delay is included. We may attempt to show that such results carry over in future work.

\subsection{Vaccination strategies}

We have seen that the disease can remain endemic. When this happens we may wish to implement some method of disease control, such as a vaccination strategy. There 
are two main types of vaccination strategy, namely a constant vaccination strategy (CVS) and a pulse vaccination strategy (PVS). These are described in subsection 7.4. We have seen in section 10.3 that, if a CVS is imposed on the model of (10.15), (10.16), and (10.17), then the model equations change into (10.20), (10.21), and (10.22). We have also seen in section 10.3 how the model of (10.15), (10.16), and (10.17) is altered by the introduction of a PVS. In the current section we shall find conditions that ensure a CVS or a PVS will eradicate the disease. First we find such conditions when there is a CVS.

Theorem 10.2. Suppose that (10.18), (10.19), and (10.25) hold. In the CVS model of (10.20), (10.21), and (10.22), assume the vaccination rate $\pi$ satisfies:

$$
\pi>1-\left(\frac{\mu+\gamma}{\beta}\right)\left(\frac{A}{B}\right)\left(\frac{M}{W}\right)^{2},
$$

where $M$ and $W$ are as defined in (10.25) and $A$ and $B$ are defined in lemma 10.2. Then $I(t) \rightarrow 0$ as $t \rightarrow \infty$.

Proof. When (10.20), (10.21), and (10.22) hold, then (10.24) holds. Then, using (10.20), (10.24), and the quotient rule for differentiation we find that:

$$
\dot{x}=\Lambda(1-\pi) \frac{N(t-\tau)}{N(t)}-x\left(\beta y+\Lambda \frac{N(t-\tau)}{N(t)}\right) \text { for } t>0,
$$

where $x=x(t)=\frac{S(t)}{N(t)}, \dot{x}=\frac{d x(t)}{d t}$, and $y=y(t)=\frac{I(t)}{N(t)}$. Notice that, since $S(t), I(t)$, and $N(t)$ satisfy strict positivity for $t \geq-\tau$, then so do $x(t)$ and $y(t)$.

Now by positivity $-\beta x y \leq 0$. Using this and using lemma 10.2 , we may deduce by (10.68) that, for $t>0$ :

$$
\dot{x} \leq \Lambda(1-\pi) B\left(\frac{W}{M}\right)-\Lambda A\left(\frac{M}{W}\right) x .
$$

It follows (theorem 1.1, pp. 78-79, [128]) that $x(t) \leq X(t)=X$ for $t \geq 0$ where $X(0)=x(0)=\frac{S(0)}{N(0)}>0$ and where:

$$
\dot{X}=\Lambda(1-\pi) B\left(\frac{W}{M}\right)-\Lambda A\left(\frac{M}{W}\right) X \text { for } t>0 .
$$

By directly solving for $X(t)$, it is trivially seen that $X(t) \rightarrow(1-\pi)\left(\frac{B}{A}\right)\left(\frac{W}{M}\right)^{2}$ as $t \rightarrow \infty$. Hence for any $\epsilon>0$, there exists $t_{\epsilon}>0$ such that, for all $t \geq t_{\epsilon}$, we have:

$$
x(t) \leq X(t) \leq(1-\pi)\left(\frac{B}{A}\right)\left(\frac{W}{M}\right)^{2}+\epsilon .
$$

Then by (10.21), we will have

$$
\frac{d I(t)}{d t} \leq\left(\beta(1-\pi)\left(\frac{B}{A}\right)\left(\frac{W}{M}\right)^{2}+\beta \epsilon-(\mu+\gamma)\right) I(t) \text { for } t \geq t_{\epsilon} .
$$


In particular this is true for $\epsilon=\left(\frac{1}{2 \beta}\right)\left((\mu+\gamma)-\beta(1-\pi)\left(\frac{B}{A}\right)\left(\frac{W}{M}\right)^{2}\right)$, which is easily seen to be positive by rearranging (10.67). But then we know that there exists $t_{\epsilon}>0$ such that, for all $t \geq t_{\epsilon}$, then

$$
\frac{d I(t)}{d t} \leq-\alpha I(t)
$$

where $\alpha=\left(\frac{1}{2}\right)\left((\mu+\gamma)-\beta(1-\pi)\left(\frac{B}{A}\right)\left(\frac{W}{M}\right)^{2}\right)>0$.

Note that $I\left(t_{\epsilon}\right)>0$ by strict positivity. It follows that $I(t) \leq I^{*}(t)$ for $t \geq t_{\epsilon}$ where $I^{*}\left(t_{\epsilon}\right)=I\left(t_{\epsilon}\right)>0$ and where, for $t \geq t_{\epsilon}$, we have $\frac{d I^{*}(t)}{d t}=-\alpha I^{*}(t)$. Solving for $I^{*}(t)$ and using the positivity of $I(t)$ we can therefore write:

$$
0 \leq I(t) \leq I^{*}(t)=I\left(t_{\epsilon}\right) e^{-\alpha\left(t-t_{\epsilon}\right)} \text { for } t \geq t_{\epsilon} .
$$

Clearly, then, $I(t) \rightarrow 0$ as $t \rightarrow \infty$, as required.

Now we find conditions on a PVS such that the disease is eradicated.

Theorem 10.3. Suppose that (10.18), (10.19), and (10.25) hold. Define the times $t_{i}$ to satisfy: $t_{1}>0$ and $t_{i+1}=t_{i}+T$ for $i \geq 1$ where $T$ is a positive constant. Suppose that (10.15), (10.16), and (10.17) hold for $t \geq 0$ except at the times $t_{i}$ where, for a constant $0 \leq p<1$, we have: $S\left(t_{i}\right)=(1-p) S\left(t_{i}^{-}\right), I\left(t_{i}\right)=I\left(t_{i}^{-}\right)$, and $R\left(t_{i}\right)=R\left(t_{i}^{-}\right)+p S\left(t_{i}^{-}\right)$where $t_{i}^{-}$is the time "momentarily" before $t_{i}$. Let $q=1-p$. Define $M$ and $W$ are as in (10.25) and define $A$ and $B$ as in lemma 10.2. Finally suppose that:

$$
\frac{\frac{K_{1}}{K_{2}}\left(1-e^{-K_{2} T}\right)\left(1+q-q e^{-K_{2} T}\right)}{1-q e^{-K_{2} T}}<\frac{\gamma+\mu}{\beta},
$$

where $K_{1}=\Lambda B\left(\frac{W}{M}\right)$ and $K_{2}=\Lambda A\left(\frac{M}{W}\right)$. Then $I(t) \rightarrow 0$ as $t \rightarrow \infty$.

It turns out that a PVS can always be found to satisfy (10.75) but we defer a more explicit discussion until after the proof.

Proof. Equation (10.24) holds for the PVS model, as noted in the paragraph directly after (10.24). Hence lemma 10.2 holds even when there is a PVS. Now observe that by (10.51) and lemma 10.2 we have:

$$
\dot{x} \leq \Lambda B\left(\frac{W}{M}\right)-x\left(\beta y+\Lambda A\left(\frac{M}{W}\right)\right) \quad \text { for } t>0, t \neq t_{i} \text { where } i \geq 1,
$$

where $x=x(t)=\frac{S(t)}{N(t)}, \dot{x}=\frac{d x(t)}{d t}$, and $y=y(t)=\frac{I(t)}{N(t)}$. Notice that, since $S(t)>0$, $I(t)>0$, and $N(t)>0$ for $t \geq-\tau$ by strict positivity, then (10.76) reveals that:

$$
\dot{x} \leq K_{1}-K_{2} x \quad \text { for } t>0, t \neq t_{i} \text { where } i \geq 1,
$$

where $K_{1}=\Lambda B\left(\frac{W}{M}\right)$ and $K_{2}=\Lambda A\left(\frac{M}{W}\right)$.

It follows that $x(t) \leq g(t)$ for $t \in\left[t_{i}, t_{i+1}\right)$ for any particular $i \geq 1$ where $g\left(t_{i}\right)=$ $x\left(t_{i}\right)=\frac{S\left(t_{i}\right)}{N\left(t_{i}\right)}>0$ and where, for $t \in\left(t_{i}, t_{i+1}\right)$ :

$$
\frac{d g(t)}{d t}=K_{1}-K_{2} g(t)
$$


Solving for $g(t)$ reveals that:

$$
x(t) \leq g(t)=\frac{K_{1}}{K_{2}}\left(1-e^{-K_{2}\left(t-t_{i}\right)}\right)+e^{-K_{2}\left(t-t_{i}\right)} x\left(t_{i}\right) \text { for } t \in\left[t_{i}, t_{i+1}\right), i \geq 1 .
$$

But then:

$$
x\left(t_{i+1}^{-}\right) \leq \frac{K_{1}}{K_{2}}\left(1-e^{-K_{2} T}\right)+e^{-K_{2} T} x\left(t_{i}\right) .
$$

But now $x\left(t_{i+1}\right)=q x\left(t_{i+1}^{-}\right)$since $S\left(t_{i+1}\right)=q S\left(t_{i+1}^{-}\right)$and $N\left(t_{i+1}\right)=N\left(t_{i+1}^{-}\right)$. Hence, by (10.80):

$$
x\left(t_{i+1}\right) \leq q \frac{K_{1}}{K_{2}}\left(1-e^{-K_{2} T}\right)+q e^{-K_{2} T} x\left(t_{i}\right) .
$$

Let $x_{i}=x\left(t_{i}\right)$. Then by (10.81):

$$
x_{i+1} \leq E+F x_{i}
$$

where $E=q \frac{K_{1}}{K_{2}}\left(1-e^{-K_{2} T}\right)$ and $F=q e^{-K_{2} T}$. Notice that $E>0$ and $0<F<1$. Iterating (10.82), we quickly find that, for $i>1$,

$$
x_{i} \leq F^{i-1} x_{1}+E \sum_{j=0}^{i-2} F^{j} .
$$

Now $0<F<1$, so the partial sum in (10.83) will be less than the entire sum, that is,

$$
\sum_{j=0}^{i-2} F^{j}<\frac{1}{1-F}
$$

Hence:

$$
\begin{aligned}
x\left(t_{i}\right)=x_{i} & \leq F^{i-1} x_{1}+\frac{E}{1-F} \\
& =\left(q e^{-K_{2} T}\right)^{i-1} x_{1}+\frac{q\left(\frac{K_{1}}{K_{2}}\right)\left(1-e^{-K_{2} T}\right)}{1-q e^{-K_{2} T}} .
\end{aligned}
$$

On the interval $t \in\left[t_{i}, t_{i+1}\right)$ it is clear that $1-e^{-K_{2}\left(t-t_{i}\right)}$ is bounded above by $1-$ $e^{-K_{2} T}$ and that $e^{-K_{2}\left(t-t_{i}\right)}$ is bounded above by 1 . But then, using (10.79) and (10.85), we can write, for $t \in\left[t_{i}, t_{i+1}\right), i \geq 1$, that:

$$
\begin{aligned}
x(t) & \leq \frac{K_{1}}{K_{2}}\left(1-e^{-K_{2} T}\right)+\left(q e^{-K_{2} T}\right)^{i-1} x_{1}+\frac{q\left(\frac{K_{1}}{K_{2}}\right)\left(1-e^{-K_{2} T}\right)}{1-q e^{-K_{2} T}} \\
& =\frac{\frac{K_{1}}{K_{2}}\left(1-e^{-K_{2} T}\right)\left(1+q-q e^{-K_{2} T}\right)}{1-q e^{-K_{2} T}}+\left(q e^{-K_{2} T}\right)^{i-1} x_{1} .
\end{aligned}
$$

Since $0<q e^{-K_{2} T}<1$ and $x_{1}=x\left(t_{1}\right)=\frac{S\left(t_{1}\right)}{N\left(t_{1}\right)}$ is finite, the term $\left(q e^{-K_{2} T}\right)^{i-1} x_{1}$ will tend to zero as $i$ increases. In other words, for any $\epsilon>0$, then $\left(q e^{-K_{2} T}\right)^{i-1} x_{1}<\epsilon$ for all $i$ big enough. So if we assume that (10.75) holds, then we can choose

$$
\epsilon=\frac{1}{2}\left(\frac{\gamma+\mu}{\beta}-\left(\frac{\frac{K_{1}}{K_{2}}\left(1-e^{-K_{2} T}\right)\left(1+q-q e^{-K_{2} T}\right)}{1-q e^{-K_{2} T}}\right)\right)>0,
$$


and we can conclude that, for $\epsilon$ given by (10.87), there exists $i^{*}>0$ such that, for $i \geq i^{*}$, then $\left(q e^{-K_{2} T}\right)^{i-1} x_{1}<\epsilon$. But then, by (10.86), we find, for $i \geq i^{*}$ :

$$
x(t)<\left(\frac{1}{2}\right)\left(\frac{\frac{K_{1}}{K_{2}}\left(1-e^{-K_{2} T}\right)\left(1+q-q e^{-K_{2} T}\right)}{1-q e^{-K_{2} T}}+\frac{\gamma+\mu}{\beta}\right) \text { for } t \in\left[t_{i}, t_{i+1}\right) .
$$

But then, since $x(t)=\frac{S(t)}{N(t)}$, we find, by equation (10.16), that $\frac{d I(t)}{d t}<-\alpha I(t)$ for $t \in\left[t_{i}, t_{i+1}\right)$ for all $i \geq i^{*}$, where

$$
\alpha=\left(\frac{\beta}{2}\right)\left(\frac{\gamma+\mu}{\beta}-\frac{\frac{K_{1}}{K_{2}}\left(1-e^{-K_{2} T}\right)\left(1+q-q e^{-K_{2} T}\right)}{1-q e^{-K_{2} T}}\right)>0 .
$$

Since $I(t)$ is unchanged at each pulse, it follows that $\frac{d I(t)}{d t}<-\alpha I(t)$ for all $t \geq t_{i^{*}}$. But then it also follows that $I(t) \leq g(t)$ for $t \geq t_{i^{*}}$ where $g\left(t_{i^{*}}\right)=I\left(t_{i^{*}}\right)$ and $\frac{d g(t)}{d t}=$ $-\alpha g(t)$ for $t>t_{i^{*}}$. By strict positivity, $I\left(t_{i^{*}}\right)>0$. Then, solving for $g(t)$ and given that $I(t)$ satisfies positivity, we have:

$$
0 \leq I(t) \leq g(t)=I\left(t_{i^{*}}\right) e^{-\alpha\left(t-t_{i^{*}}\right)} \text { for } t \geq t_{i^{*}} .
$$

Hence, since $\alpha>0$, we have $I(t) \rightarrow 0$ as $t \rightarrow \infty$.

Now let us comment on how restrictive condition (10.75) is. If we set $G=\frac{\left(\frac{\gamma+\mu}{\beta}\right)}{\left(\frac{K_{1}}{K_{2}}\right)}>$ 0 , then the condition may be written:

$$
\frac{\left(1-e^{-K_{2} T}\right)\left(1+q-q e^{-K_{2} T}\right)}{1-q e^{-K_{2} T}}<G .
$$

Note first of all that if $0<q<1$, then (10.90) clearly holds when $T=0$. Also the left hand side in (10.90) is a continuous function of $T$ for $T \geq 0$, so there will exist a range of $T>0$ such that (10.90) holds. In other words, a PVS can always be found to satisfy (10.90), although we have yet to comment on how small $T$ might have to be.

Secondly note that if $q=1$, then (10.90) becomes $2-e^{-K_{2} T}<G$. Clearly, then, if $G>2$ then a PVS with $q=1$ will succeed. But if $q=1$, then $p=1-q=0$, so that the PVS effectively does nothing. Hence when $G>2$ we can say that the disease must be going extinct naturally and that imposing a PVS does nothing to alter this fact. Notice that $G>2$ amounts to an upper bound on the contact rate $\beta$, since $G$ is inversely proportional to $\beta$. In fact if $G>2$ then $\beta<\mu+\gamma$, in which case lemma 10.1 ensures that $I(t) \rightarrow 0$ as $t \rightarrow \infty$.

Suppose again that $0<q<1$. Given such a $q$ we now find a $T^{*}>0$ such that the PVS will succeed if $0<T<T^{*}$. Let $X=e^{-K_{2} T}$ and note that, since $T>0$, we must have $0<X<1$. If we define $f_{1}(X)=(1-X)(1+q-q X)$ and $f_{2}(X)=G(1-q X)$, then (10.90) becomes:

$$
f_{1}(X)=(1-X)(1+q-q X)<G(1-q X)=f_{2}(X) .
$$

The trivial forms of the functions $f_{1}(X)$ and $f_{2}(X)$ make it easy to determine when (10.91) holds for $0<X<1$. A few simple sketches (one of which is given in figure 10.1) quickly reveal that, for $G>0$ and $0<q<1$, then: 


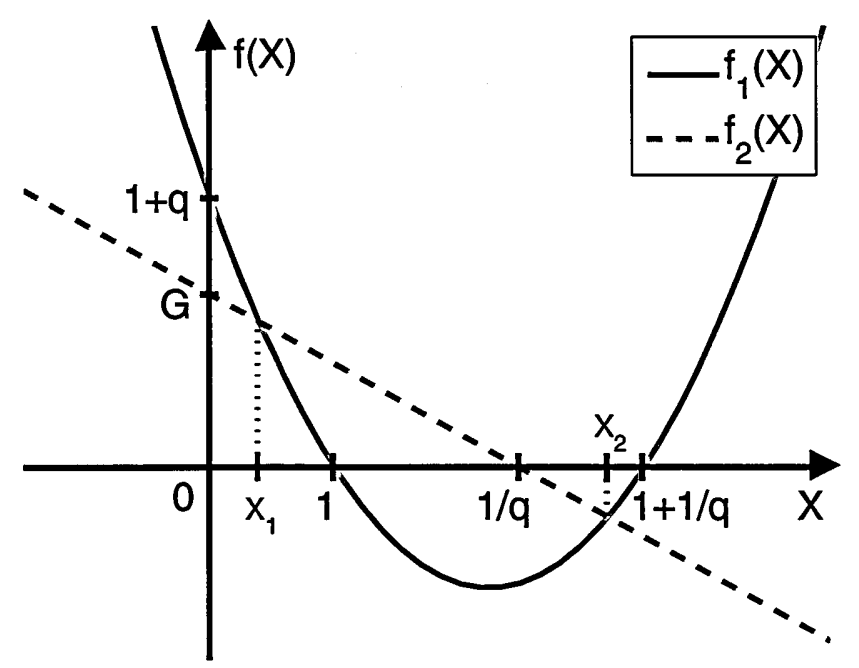

Figure 10.1: Comparison of left and right hand sides of (10.91) when $G<1+q$. Here $q=0.4$ and $G=0.9$.

(A1) $f_{1}(X)=f_{2}(X)$ has two solutions, namely $X_{1}$ and $X_{2}$ where $X_{1}<1<X_{2}$.

(A2) $f_{1}(X)<f_{2}(X)$ for $X_{1}<X<1$.

(A3) $X_{1}<0$ if $G>1+q$, which is true in particular if $G>2$.

(A4) $X_{1}>0$ if $G<1+q$, which is true in particular if $G<1$.

Since $f_{1}(X)=f_{2}(X)$ is a quadratic equation, we may find $X_{1}$ and $X_{2}$ explicitly:

$$
X_{1,2}=\frac{(1+2 q-q G) \pm \sqrt{ }\left\{(1+2 q-q G)^{2}-4 q(1+q-G)\right\}}{2 q} .
$$

Results (A1) to (A4) may be proven by using the inequalities $0<q<1$ and $G>0$ in (10.92). We will not include the actual calculations since the results are obvious geometrically.

Now by (A2), (A3), and (A4), we can say that (10.91) will hold when $X^{*}<X<1$ where:

$$
X^{*}= \begin{cases}0 & \text { if } G>1+q \\ X_{1} & \text { if } G<1+q .\end{cases}
$$

Hence (10.90) will hold for any $q$ and $T$ with $0<q<1$ and $0<T<T^{*}$ where:

$$
T^{*}= \begin{cases}\infty & \text { if } G>1+q \\ -\frac{1}{K_{2}} \ln \left(X_{1}\right) & \text { if } G<1+q .\end{cases}
$$

Notice by (A1) and (A4) that $0<X_{1}<1$ if $G<1+q$, so that $T^{*}>0$ in (10.94). We may conclude that a successful PVS exists no matter what strength the pulses are, as long as they do something (so that $0<q<1$ ), and the inter-pulse time $T$ is small enough. 

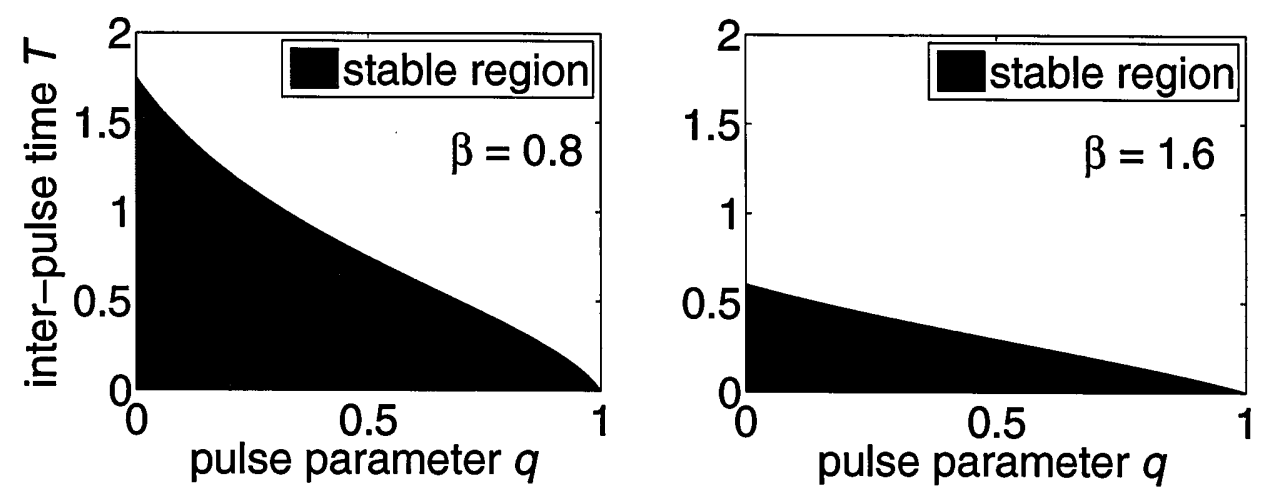

Figure 10.2: Successful PVS regions shaded (see section 10.6). Model parameters: $\Lambda=2, \mu=1, M=1, W=1.3, \gamma=1$. Hence also $A=\min \left(1, \frac{\mu}{\Lambda}\right)=0.5$ and $B=\max \left(1, \frac{\mu}{\Lambda}\right)=1$.

\subsection{Simulations}

In figure 10.2 we plot the region in the $q-T$ plane guaranteed by theorem 10.3 to ensure a PVS will successfully eradicate the disease absolutely in two cases, each with a different value of $\beta$. These regions are called stable regions in the plots. The region in each plot is the set of points $(q, T)$ where $0<q<1$ and $0<T<T^{*}$ where $T^{*}$ satisfies (10.94). Note that $T^{*}$ can be considered a function of $q$.

Observe that the stable region becomes smaller as $\beta$ increases. This is intuitively sensible because, if the contact rate $\beta$ increases, we would expect the disease to spread more easily, so that a stronger vaccination strategy would be needed to bring it under control. Note that a strong PVS will be near the origin, since a PVS is stronger when $T$ is smaller and when $p$ is larger, making $q=1-p$ smaller.

In figure 10.3 we plot the number of infectives in three cases, namely: in the absence of vaccination; when there is a CVS; and when there is a PVS. The CVS is chosen to be just strong enough by theorem 10.2 to succeed in absolute disease eradication. The PVS is chosen as the weakest possible (for a given pulse strength $p=0.5$ ) guaranteed by theorem 10.3 to deliver absolute disease eradication.

\subsection{Insect pathogen dynamics}

Infectious diseases are by no means suffered only by mammals. In particular, insects can be infected by viral, bacterial, fungal, protozoan, and nematode agents [77]. The study of insect pathogens (also called entomopathogens [97]) is an emerging field [56, $58,111,136,138]$, driven by its main application - pest control.

Pathogens can be introduced as biological control agents to reduce populations of insect pests, and some real-world introductions have been seen as a cost-effective alternative to chemical pesticides [97]. Microbial control agents, if investigated sensibly prior to introduction into a natural ecosystem, have other advantages over pesticides including: safety for humans and non-target organisms, reduction of pesticide 

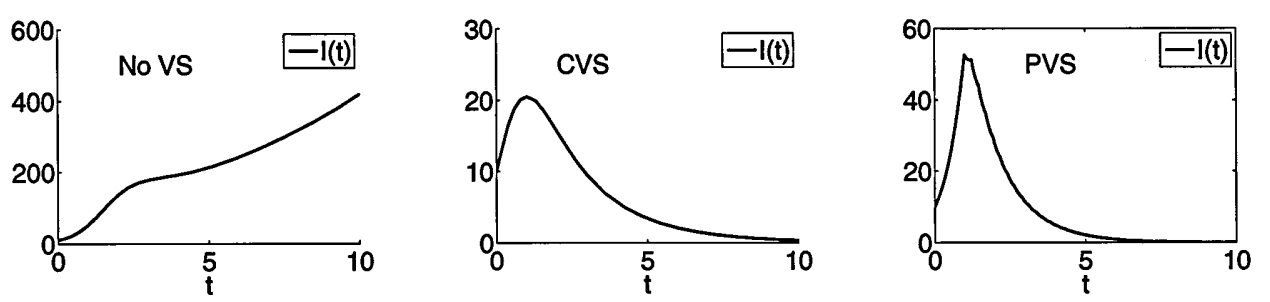

Figure 10.3: Number of infectives, with no VS, with CVS, or with PVS. Initial data: $(S(t), I(t), R(t))=(400,10,10)$ for $t \in[-\tau, 0]=[-1,0]$ (so that $\frac{M}{W}=\frac{W}{M}=1$ ). Model parameters: $\Lambda=1.3$ and $\mu=1$ (so that $A=0.769$ and $B=1$ ). Also $\gamma=1, \beta=4$. CVS strength $\pi=0.6154$ is chosen to just satisfy (10.67). PVS parameters: $t_{1}=1, q=0.5$ (so that $p=0.5$ ) and $T=0.2365$ is chosen to satisfy (10.94).

residues in food, and preservation of a pest's natural enemies.

Suppose that a particular insect species has a life cycle with distinct stages. Suppose further that a disease circulates in only one of these stages in a population of such an insect. This is not unrealistic - if the developmental stages are distinct in form, diet, and habitat, then a disease infecting one stage may not infect the other, quite different stages. When a model is derived to describe the dynamics of such a disease, it may involve a "gestation" delay if maturation is considered as a type of gestation. We will consider two insect pathogen models in this section.

Understanding when an entomopathogen may or may not eradicate a pest insect is an area of pest control, so the work in this section could be included in the pest control part of this thesis. However, the knowledge that an entomopathogen may eradicate a species of economic importance to us, including pollinators such as bees, is likely to prompt us to seek ways to control the spread of the disease and to conserve the beneficent species. In this sense, insect pathogen dynamics is an area of disease control. Ultimately, it is because the models of this section are precisely those described by the heading of the present chapter - epidemiological models with a gestation delay - that we include them here.

\subsubsection{Two models}

We derive models for an insect population which has two life stages - juvenile (or immature) and adult. The process of deriving them will be similar to that used in section 10.2, with foetuses and born individuals in section 10.2 corresponding to juveniles and adults here. Henceforth an individual will mean an insect of any age $a \geq 0$.

Let $u(t, a)$ be the density of individuals of age $a$ at time $t$, and assume that an individual becomes mature on reaching age $\tau$ where $\tau>0$ is a constant. Then $u(t, \tau)$ is the rate at which individuals pass through age $\tau$ and therefore represents adult recruitment. Assume that an infectious disease with SIR-type dynamics is present in the adult population. The juvenile stage does not and cannot suffer from the disease. Let $S(t), I(t)$, and $R(t)$ denote the number of susceptible, infective, and removed adults respectively. We shall write $S, I$, and $R$ as shorthands for $S(t), I(t)$, and $R(t)$. The total adult population is $N=N(t)=S(t)+I(t)+R(t)$. 
Define $\mu_{s}$ to be the per capita death rate of susceptibles and removeds, $\mu_{i}$ to be the per capita death rate of infectives, and $\gamma$ to be the per capita infective recovery rate. Let $\mu_{s}, \mu_{i}$, and $\gamma$ be positive constants. Recovery is assumed to confer permanent immunity. Let the disease spread by standard incidence (see subsection 8.1.1) with average per adult contact rate $\beta$ where $\beta>0$ is a constant. We assume a "contact" to be sufficient for disease transmission if it is between an infective and a susceptible. Then we may write:

$$
\begin{aligned}
\frac{d S(t)}{d t} & =u(t, \tau)-\beta \frac{S(t) I(t)}{N(t)}-\mu_{s} S(t) \\
\frac{d I(t)}{d t} & =\beta \frac{S(t) I(t)}{N(t)}-\left(\gamma+\mu_{i}\right) I(t) \\
\frac{d R(t)}{d t} & =\gamma I(t)-\mu_{s} R(t),
\end{aligned}
$$

Notice that (10.95), (10.96), and (10.97) are identical in form to (10.2), (10.3), and (10.4).

Now let us find an expression for $u(t, \tau)$. Assume that the juveniles are governed by the standard McKendrick-von Foerster model for an age-structured population. Then, if the juvenile per capita death rate is a constant $\mu_{j}>0$, we may write:

$$
\frac{\partial u}{\partial t}+\frac{\partial u}{\partial a}=-\mu_{j} u, \quad t>0, \quad 0<a<\tau .
$$

where $u$ is a shorthand for $u(t, a)$. Assume we have an initial condition:

$$
u(0, a)=u_{0}(a) \geq 0, \quad a \geq 0 .
$$

Also assume that the birth rate, which is $u(t, 0)$, is a function of the total number of adults, so that:

$$
u(t, 0)=b(N(t)) \text {. }
$$

Solving for $u(t, a)$ and then setting $a=\tau$ yields:

$$
u(t, \tau)=e^{-\mu_{j} \tau} b(N(t-\tau)) .
$$

Technically this latter expression is only valid for $t>\tau$ but we shall follow the convention in the literature and assume for our model that (10.101) holds for $t>0$. (See [125] for some subtle comments here.) Our model for the adult population is, then, for $t>0$,

$$
\begin{aligned}
\frac{d S(t)}{d t} & =e^{-\mu_{j} \tau} b(N(t-\tau))-\beta \frac{S(t) I(t)}{N(t)}-\mu_{s} S(t) \\
\frac{d I(t)}{d t} & =\beta \frac{S(t) I(t)}{N(t)}-\left(\gamma+\mu_{i}\right) I(t) \\
\frac{d R(t)}{d t} & =\gamma I(t)-\mu_{s} R(t) .
\end{aligned}
$$

To be a sensible model, we require initial data. Therefore we shall additionally assume that:

$$
S(t)=S_{0}(t) \geq 0, \quad I(t)=I_{0}(t) \geq 0, \quad R(t)=R_{0}(t) \geq 0 \quad \text { for } t \in[-\tau, 0] .
$$

We shall analyse this model in the next subsection. 
Now let us derive a model with SI-type dynamics. Once again we consider a disease circulating in the adult stage of an insect but now assume that there is no recovery from infection. A suitable model is:

$$
\begin{aligned}
\frac{d S(t)}{d t} & =u(t, \tau)-\beta \frac{S(t) I(t)}{N(t)}-\mu_{s} S(t) \\
\frac{d I(t)}{d t} & =\beta \frac{S(t) I(t)}{N(t)}-\mu_{i} I(t) .
\end{aligned}
$$

where the parameters $\beta, \mu_{s}$, and $\mu_{i}$ are as defined in the model of (10.102), (10.103), and (10.104), and where we now have $N(t)=S(t)+I(t)$.

Suppose again that the juveniles are governed by the McKendrick-von Foerster model (equation (10.98)) and that the initial condition (10.99) holds. But now suppose that the infection is serious enough to prevent infectives from reproducing. Then it becomes appropriate to think of the birth rate as a function of the total number of susceptibles, so that $u(t, 0)=b(S(t))$. Solving for $u(t, a)$ and then setting $a=\tau$ now yields:

$$
u(t, \tau)=e^{-\mu_{j} \tau} b(S(t-\tau)) .
$$

Therefore our model becomes:

$$
\begin{aligned}
\frac{d S(t)}{d t} & =e^{-\mu_{j} \tau} b(S(t-\tau))-\beta \frac{S(t) I(t)}{N(t)}-\mu_{s} S(t) \\
\frac{d I(t)}{d t} & =\beta \frac{S(t) I(t)}{N(t)}-\mu_{i} I(t) .
\end{aligned}
$$

Once again, to be a sensible model we require initial data, so we additionally assume that:

$$
S(t)=S_{0}(t) \geq 0, \quad I(t)=I_{0}(t) \geq 0 \quad \text { for } t \in[-\tau, 0] .
$$

Assuming the birth functions are sensible in the two models derived in this subsection, then both models have unique solutions for $t \geq 0$ and satisfy positivity (that is, $S(t) \geq 0, I(t) \geq 0$, and $R(t) \geq 0$ for $t \geq 0$ in the model of (10.102), (10.103), and (10.104) and $S(t) \geq 0, I(t) \geq 0$ for $t \geq 0$ in the model of (10.109) and (10.110)). (See [95] for results on existence and uniqueness of solutions and page 81 of [128] for a result on positivity.)

\subsubsection{Model analyses}

Let us now consider the model defined by (10.102), (10.103), (10.104), and (10.105). Suppose first of all that the birth function is linear with $b(N)=\lambda N$ (where $\lambda$ is a positive constant) and that the disease is non-fatal, so that $\mu_{i}=\mu_{s}$. Then if we let $\Lambda=$ $e^{-\mu_{j} \tau} \lambda$ and $\mu=\mu_{i}=\mu_{s}$, we recover the model for a non-fatal mammalian disease defined by (10.15), (10.16), (10.17), and (10.18). Thus the results of section 10.4 will hold. (The results of section 10.5 concerning vaccination strategies will also hold but we will not dwell on this since insects are seldom subjected to vaccination programs in the real world.) In particular, then, we see that the disease will be endemic if condition (10.44) holds.

Even though a non-fatal disease which does not hinder reproduction is unlikely to be an effective pest control measure or a threat to an economically valuable creature such as the honey bee, it is nevertheless of interest to observe that an insect pathogen 
model and a mammalian disease model can be qualitatively identical. This prompts us to suggest that the insect pathogen models in the literature $[56,111,138]$ be viewed in a wider context henceforth for they may be appropriate not only to insects.

What happens in the model of (10.102), (10.103), (10.104), and (10.105) when there are disease-caused deaths? Notice by (10.103) that if $\beta<\gamma+\mu_{i}$ then, since $\frac{S(t)}{N(t)} \leq 1$ by positivity, we have $\frac{d I(t)}{d t}<0$ for $t>0$, and indeed we can deduce that $I(t) \rightarrow 0$ as $t \rightarrow \infty$ by a trivial argument. This warns us that if we wish to introduce an insect pathogen as a pest control measure then we should not necessarily choose the most lethal agent at our disposal, for if the disease death rate $\mu_{i}$ is high enough that $\mu_{i}+\gamma>\beta$, then the disease will die out and will cease to exert any controlling influence. To lend credence to this warning we should prove that the total population of the creature need not die out when $I(t) \rightarrow 0$. This is achieved in the following theorem:

Theorem 10.4. In the model of (10.102), (10.103), (10.104), and (10.105), suppose that $S_{0}(t) \geq M$ for $t \in[-\tau, 0]$ where $M$ is a positive constant, and that $b(N)=\lambda N$ where $\lambda$ is a positive constant. If $e^{-\mu_{j} \tau} \lambda>\beta+\mu_{s}$, then $S(t) \rightarrow \infty$ as $t \rightarrow \infty$.

Proof. By positivity $\frac{I(t)}{N(t)} \leq 1$ and $N(t-\tau) \geq S(t-\tau)$ for $t>0$. But then, by (10.102), we can write,

$$
\frac{d S(t)}{d t} \geq e^{-\mu_{j} \tau} \lambda S(t-\tau)-\left(\beta+\mu_{s}\right) S(t) \text { for } t>0 .
$$

But then (using theorem 1.1, pp. 78-79, [128]) we can say that $S(t) \geq S_{1}(t)$ for $t \geq-\tau$ where $S_{1}(t)=S_{0}(t) \geq M$ for $t \in[-\tau, 0]$ and where

$$
\frac{d S_{1}(t)}{d t}=e^{-\mu_{j} \tau} \lambda S_{1}(t-\tau)-\left(\beta+\mu_{s}\right) S_{1}(t) \text { for } t>0 .
$$

By theorem 3.3 and the assumption that $e^{-\mu_{j} \tau} \lambda>\beta+\mu_{s}$, we deduce that $S_{1}(t) \rightarrow \infty$ as $t \rightarrow \infty$. But then $S(t) \geq S_{1}(t) \rightarrow \infty$ as $t \rightarrow \infty$.

Besides its value in combination with the comments immediately prior to it, theorem 10.4 has another disturbing consequence. The condition that $e^{-\mu_{j} \tau} \lambda>\beta+\mu_{s}$ is independent of the disease death rate $\mu_{i}$, so that, if this condition holds, then the susceptible population will explode regardless of the virulence of an introduced pathogenic agent. Of course a linear birth function may only be appropriate for a small population (see subsection 3.2.1), but if this is so then theorem 10.4 is still disturbing, for it suggests that a small pest population will not necessarily remain small whether or not a pathogen is introduced. Thus there may be circumstances in which insect pathogens are not a viable control option.

Further evidence that insect pathogen introductions should be made carefully is provided by figure 10.4, which shows that the average population of an insect can be increased by an SIR disease. In figure 10.4 we plot the insect population in the absence of disease $(N(t))$ and in the presence of disease $\left(N^{*}(t)\right)$. To plot the population $N^{*}(t)$ in the presence of disease, we simulate the SIR model of (10.102), (10.103), (10.104), and (10.105) with the initial data $(S(t), I(t), R(t))=(20,40,30)$ for $t \in[-\tau, 0]$ where $\tau=1$, and with $\mu_{s}=0.5, \mu_{i}=16.5, \mu_{j}=0.1, \beta=20, \gamma=1$, and where the birth function is of Allee type (see subsection 3.2.1) and satisfies $b(N)=10 N^{2} e^{-0.1 N}$. We acquire the relevant population model in the absence of disease when we set $I(t)=0$, 


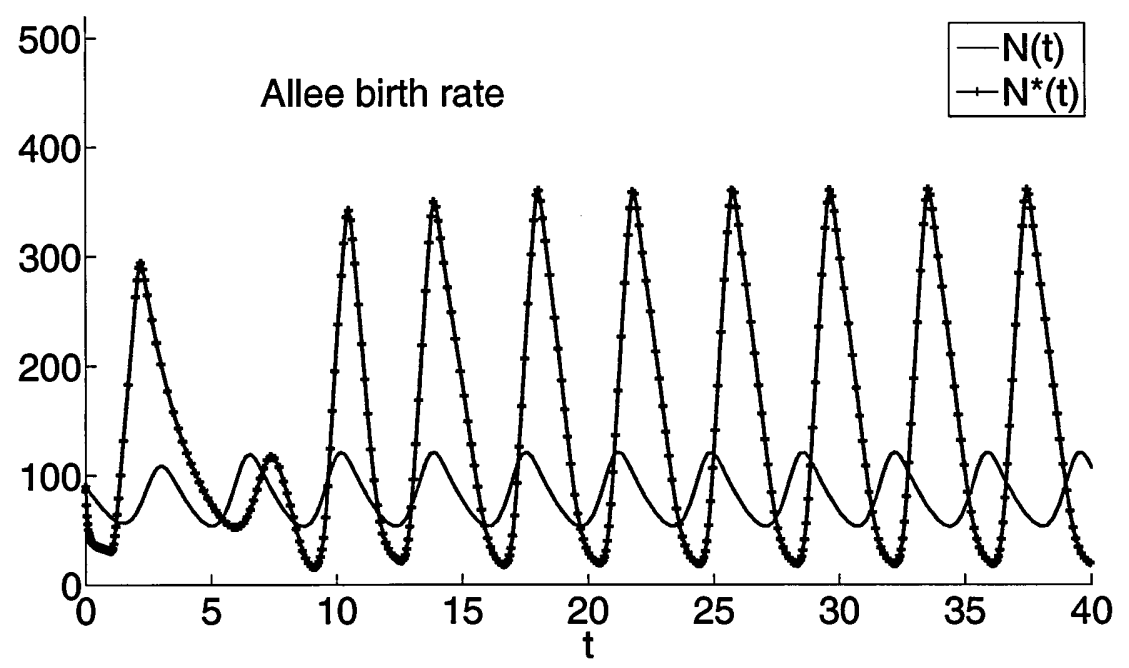

Figure 10.4: An insect population in the absence of disease $(N(t))$ and when a disease with SIR dynamics is present $\left(N^{*}(t)\right)$. See subsection 10.7 .2 for details.

$R(t)=0$, and $S(t)=N(t)$ in the model of (10.102), (10.103), (10.104), and (10.105), easily finding that:

$$
\frac{d N(t)}{d t}=e^{-\mu_{j} \tau} b(N(t-\tau))-\mu_{s} N(t) \text { for } t>0 .
$$

Therefore, to plot the population $N(t)$ in the absence of disease, we simulate (10.114) with the same initial data for the total population $(N(t)=90$ for $t \in[-\tau, 0]$ where $\tau=1)$, parameter values, and birth function as in the simulation with the disease present.

Of course we would not expect an insect population to always be increased by the presence of a fatal disease, and indeed figure 10.5 suggests that the SIR disease of (10.102), (10.103), (10.104), and (10.105) can decrease the average population in certain circumstances.

The graphs in figure 10.5 are created in the same way as the graph in figure 10.4. In the left graph of figure 10.5, we use the initial data $(S(t), I(t), R(t))=(20,40,30)$ for $t \in[-\tau, 0]$ where $\tau=1$; we choose model parameters $\mu_{s}=1, \mu_{i}=9, \mu_{j}=0.1, \beta=15$, $\gamma=2$; and we choose for the birth function to be linear and to satisfy $b(N)=1.5 N$. The left graph shows that the disease not only reduces the population but apparently drives it to extinction when, in the absence of the disease, it would steadily grow.

The middle graph in figure 10.5 is constructed in the same way as the left graph with identical initial data and model parameters, except that $\mu_{i}=5$ and the birth function is of Nicholson type (see subsection 3.2.1) and satisfies $b(N)=10 N e^{-0.03 N}$. Here the disease reduces the population but does not drive it to extinction.

The right graph in figure 10.5 is also constructed in the same way as the left graph with identical initial data. The model parameters in the right graph are $\mu_{s}=1$, $\mu_{i}=16, \mu_{f}=0.1, \beta=40, \gamma=0.5$, and the birth function is of Allee type and satisfies $b(N)=20 N^{2} e^{-0.4 N}$. Notice how the right graph shows that, for an Allee 

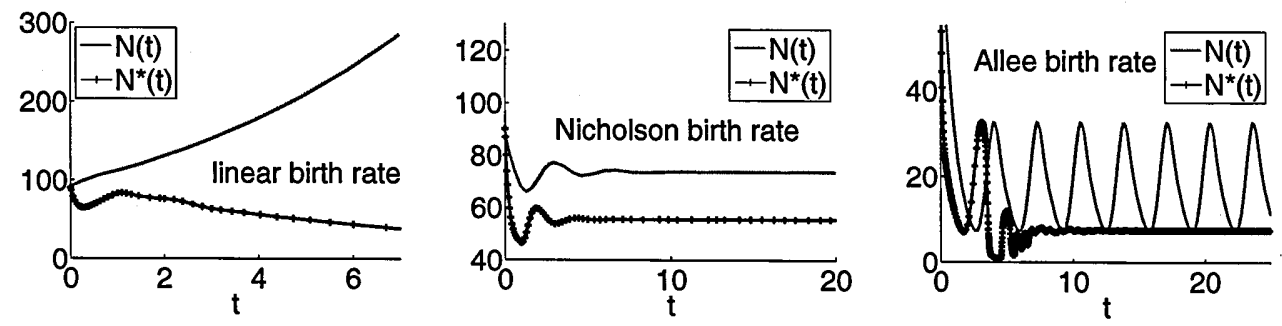

Figure 10.5: In each graph, we show an insect population in the absence of disease $(N(t))$ and when a disease with SIR dynamics is present $\left(N^{*}(t)\right)$. See subsection 10.7.2 for details.

birth function, an SIR disease can alter the behaviour of an insect population from oscillatory to constant.

A fuller investigation of the model of (10.102), (10.103), (10.104), and (10.105) is left as a matter for future research.

Finally we consider the SI model given by (10.109), (10.110), and (10.111). This is similar to an insect pathogen model for an adult-stage disease studied numerically by Moerbeek and van den Bosch [111], except that they formulate their model in terms of the densities of susceptibles and infectives rather than the numbers.

By reasoning along similar lines as in the comments immediately prior to theorem 10.4, we can say that, if $\beta<\mu_{i}$ in the model of (10.109), (10.110), and (10.111), then $I(t) \rightarrow 0$ as $t \rightarrow \infty$. Also by arguing along lines similar to the proof of theorem 10.4, we find that $S(t) \rightarrow \infty$ as $t \rightarrow \infty$ if the birth function is linear with per capita birth rate $\lambda$ and if $e^{-\mu_{j} \tau} \lambda>\beta+\mu_{s}$. Therefore the warning (stated just before theorem 10.4) that introducing an excessively virulent pathogenic agent may be ineffective as a control measure again applies, and so does the suggestion (stated just after theorem 10.4) that a pathogenic agent may be ineffective regardless of its virulence if the birth rate is sufficiently high.

Although Moerbeek and van den Bosch deduce by their simulations that the control of insect pests for which the adult stage is harmful to a crop is feasible for either an adult or a juvenile disease by increasing the transmission coefficient [111], we question if this is a practical control measure. The transmission coefficient in the models of Moerbeek and van den Bosch is the same as the contact rate if we define a contact to be sufficient for disease transmission (see subsection 10.7.1), and it must be asked if it is really within our powers to alter the social behaviour of an insect. We cannot ask a group of insects to increase their interactions any more than we can ask them to stop eating our crops. Of course a contact is arguably more likely to be sufficient for disease transmission if the infectious agent is highly virulent, but even if every possible direct contact between an infective and a susceptible results in a new infection, the rate of new infections is still restricted by the contact rate.

As in the insect pathogen model for an SIR disease, the average population of an insect can be increased by an SI disease. In figure 10.6 we plot the insect population in the absence of disease $(N(t))$ and in the presence of an SI disease $\left(N^{*}(t)\right)$. To plot the population $N^{*}(t)$ in the presence of the disease, we simulate the model of (10.109), (10.110), and (10.111) with the initial data $(S(t), I(t))=(20,30)$ for $t \in[-\tau, 0]$ where 


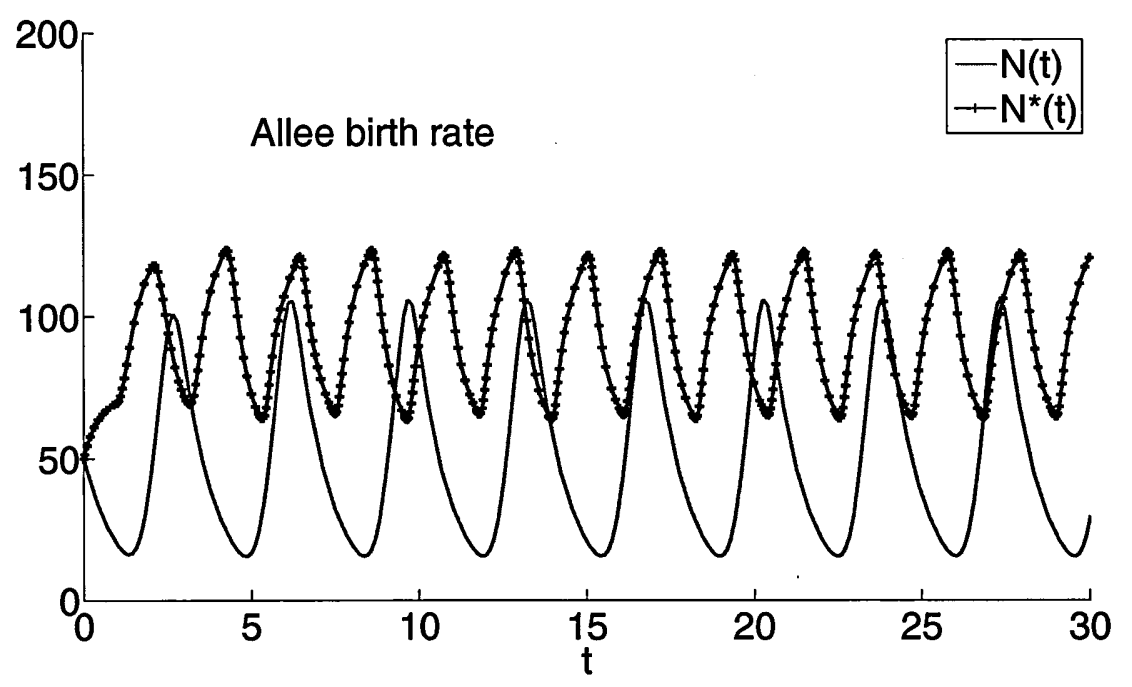

Figure 10.6: An insect population in the absence of disease $(N(t))$ and when a disease with SI dynamics is present $\left(N^{*}(t)\right)$. See subsection 10.7.2 for details.

$\tau=1$, and with $\mu_{s}=1, \mu_{i}=2, \mu_{j}=0.1, \beta=18$, and where the birth function is Allee and satisfies $b(N)=20 N^{2} e^{-0.2 N}$. We obtain the appropriate population model in the absence of disease when we set $I(t)=0, R(t)=0$, and $S(t)=N(t)$ in the model of (10.109), (10.110), and (10.111), immediately finding the non-disease model to satisfy (10.114). Therefore, to plot the population $N(t)$ in the absence of disease, we simulate (10.114) with the same initial data for the total population $(N(t)=50$ for $t \in[-\tau, 0]$ where $\tau=1)$, parameter values, and birth function as in the simulation with the SI disease present. Figure 10.6 is quite compelling. Intuition would not suggest that a fatal disease which cripples reproduction in infected individuals can actually increase the average value of a population. An explanation for this behaviour could be sought in terms of the shape of the birth function, for such an approach was fruitful when we discussed the fact in chapter 4 that infrequent culling can benefit a pest.

An insect population is not always increased by the SI disease of (10.109), (10.110), and (10.111), as we illustrate in figure 10.7. We create the graphs in 10.7 in the same way as the graph in figure 10.6.

In the left graph of figure 10.7, we choose the initial data $(S(t), I(t))=(20,30)$ for $t \in[-\tau, 0]$ where $\tau=1$; the model parameters are $\mu_{s}=1, \mu_{i}=2, \mu_{j}=0.1$, $\beta=12$; and the birth function is linear and satisfies $b(N)=3 N$. The left graph shows that the disease not only reduces the population but seemingly causes it to die out when, in the absence of the disease, it would grow. The middle graph in figure 10.7 is constructed in the same way as the left graph with identical initial data and model parameters, except that $\mu_{i}=5$ and the birth function is of Nicholson type and satisfies $b(N)=20 N e^{-0.03 N}$. The right graph in figure 10.7 is also constructed in the same way as the left graph with identical initial data. The model parameters in the right graph are $\mu_{s}=0.4, \mu_{i}=25.4, \mu_{f}=0.1, \beta=45$, and the birth function is of Allee type and satisfies $b(N)=30 N^{2} e^{-0.3 N}$. Notice how the right graph shows, for an Allee birth function, that an SI disease can alter the behaviour of an insect population from 

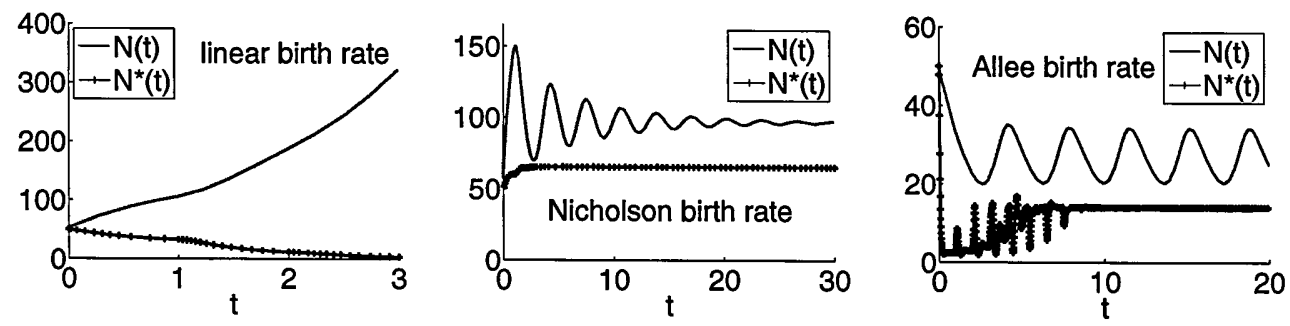

Figure 10.7: In each graph, we show an insect population in the absence of disease $(N(t))$ and when a disease with SI dynamics is present $\left(N^{*}(t)\right)$. See subsection 10.7.2 for details.

oscillatory to constant.

A more complete examination of the model of (10.109), (10.110), and (10.111) is a matter for future research.

\subsection{Discussion}

We have formulated a model for an infectious mammalian disease with SIR-type dynamics, disease-caused deaths, no vertical transmission, a constant contact rate, and a delay in the birth rate representing the natural gestation period of the host. We analysed this model in the special case when the disease is non-fatal and the conception function is linear. It was discovered that if the contact rate were high enough compared to conception and recovery rates then the disease would remain endemic. This motivated us to construct constant and pulse vaccination strategies that succeed in eradicating the disease. Simulations corroborated our results.

We also derived two insect pathogen models, one of which was shown to reduce to our non-fatal mammalian SIR model in certain circumstances. This prompted us to suggest that existing models in the literature of insect pathogen dynamics be viewed in a wider context for they may yield applications to mammalian populations. For both our insect pathogen models, we found rigorously that the population may grow regardless of the virulence of the pathogenic agent and we therefore deduced that entomopathogens may not always be appropriate as a form of biocontrol. Simulations established that the insect population may be either increased or decreased by disease, depending on the choice of model parameters.

Future research could involve analysing our mammalian SIR model when diseasecaused deaths are allowed as well as seeking a rigorous proof that an entomopathogen can increase the average population of an insect. It may also be of value to look for mammalian applications for existing entomopathogen models. 


\section{Part III}

\section{Concluding remarks}


MATERIAL REDACTED AT REQUEST OF UNIVERSITY 


\title{
Chapter 11
}

\section{Concluding remarks}

\author{
"A witty saying proves nothing." \\ Voltaire (1694 - 1778)
}

We began with the intention of extending our understanding of pest control and disease control. This has been achieved through the formulation and analysis of various mathematical models. Comments have been made on the implications of our results in terms of which real-world decisions to take.

\subsection{Pest control summary}

In our pest control work, we have examined models for a creature, such as an insect, with a life cycle consisting of two stages, namely immature and adult. When the creature lives in a single region or "patch" and the birth function is one of three different kinds (linear, Nicholson, or Allee), we have found conditions on the model parameters such that the creature naturally remains endemic or naturally goes extinct. To eradicate the pest when it would naturally be endemic, we have constructed adult impulsive culling regimes. A central finding is that only a finite number of culls is required to eradicate the pest when the birth function is of an Allee kind.

We have also found natural endemicity and extinction conditions when the creature inhabits two patches between which individuals may migrate. To eradicate the pest on both patches simultaneously when it would naturally be endemic, we have constructed adult impulsive culling regimes where the regime on one patch is carried out in a manner essentially independent from the regime on the other patch. This construction was deliberate because the patches could represent, for instance, farms with different owners where each owner would have autonomy in their pest control decisions. It was found, analogous to the single-patch model, that only a finite number of culls is needed on each patch to eradicate the pest on both patches when the birth functions on both patches are of Allee type.

Given that practical applications of our results involve using pesticides, we have recognised our responsibility to urge caution and discussed the drawbacks associated 
with their use, including environmental drawbacks. Thus we have drawn attention, for example, to the negative consequences, such as pesticide resistance, of excessively frequent applications of pesticides. However, by considering a model with a step birth function we have made a case that culling infrequently may, in some circumstances, increase the average population of a pest.

It becomes difficult to know what level of pesticide use to advocate. If none is used, the pest may destroy an entire crop; if a little is used it may, perversely, increase the average population of the pest; if too much is used, the pest may quickly develop a resistance or there may be secondary pest outbreaks. If a real-world pest infestation is sufficiently serious that pesticides are deemed necessary, we would recommend, at the very least, that careful simulations be performed before a decision is made on the most appropriate level of application. There is no reason to conclude from our work that pesticides cannot function as an efficient control method if applied sensibly.

\subsection{Pest control extensions}

All of our models have made an assumption of year-round breeding, making them appropriate for tropical pests. However, the models may also be appropriate for temperate pests during the summer breeding months. If this is true for a particular temperate pest with an Allee-type birth function, then there may be situations in which the pest would be wiped out completely in a single breeding season if we cull often enough to keep the adult population beneath its minimum viable threshold. To verify such a claim, we could formulate models for temperate pests. Temperate pests can overwinter as eggs, larvae, pupae, or adults depending on the species, so to formulate a model for a particular species, we would have to take into account the specific form of overwintering. We have already privately created a model for a temperate pest with adult overwintering and will be analysing it in due course.

The models in our pest control work have assumed that the life cycle of the pest has two stages, or may be approximated by two stages, each with a simple death function. But such an approximation will be inaccurate for many insects. An insect in the Endopterygota [17] has four stages in its life cycle (egg, larva, pupa, imago) and it will not always be sensible to lump together the first three stages into a single "immature" stage with a linear death function. Future research could involve constructing and investigating models for creatures with three or four distinct developmental stages with more complicated death functions. Research in this direction has already been started [83] but there is such a rich diversity in insect population dynamics that new questions could easily be asked.

The conditions that we have established to ensure our culling regimes succeed have amounted to requiring the regimes to be sufficiently strong. Thus, they will succeed if consecutive culls occur close enough in time. In reality, a farmer may only apply pesticides if he notices that a pest has become especially abundant. To reflect such a scenario, we could suppose that the times of the culls are responsive to the population of the pest - a cull would only occur if the population grew above a certain threshold, which would be the maximum level of infestation that the farmer is prepared to tolerate. An alternative way to modify our work would be to let the cull strengths be a function of the frequency of recent culls, the idea being that if culls occur more frequently then cull strengths will fall because pesticide resistance will grow. 
Our culling regimes have involved impulsively reducing adult numbers. But other sorts of regime may be implemented. A control method need not target only the adults and need not be impulsive. For example, culling adults continuously in time but at discrete points in space has been explored by Simons and Gourley [125] and impulsive culling of larval mosquitoes in a model for the spread of West Nile virus has been investigated by Gourley, Liu, and Wu [81]. Problems yet to be considered (to our knowledge) include larval culling, or the culling of adults continuously in time but at discrete points in space, in a model for a pest inhabiting two patches.

Another potentially fruitful problem would be the formulation and analysis of pest control models for a creature living on $n \geq 3$ patches. It could also be of value if we tried to further understand when infrequent culling can increase the average population of a pest.

Finally we could study pest control with a view to conservation. When some large mammals become very numerous, they are culled to prevent them from disrupting their ecosystem. Examples include red deer in Scotland [23,135] and elephants in South Africa [114]. The purpose of culling such animals is to control their numbers without eradicating them. The models of chapter 3 could describe the population of such a species with the delay $\tau$ representing the gestation period or time to maturity. The task would be to find a culling regime that maintains the population between a lowest acceptable level (the animal must not be eradicated) and a highest acceptable level (the animal must not be too numerous). We have attempted to find such a regime but have not yet found anything worth reporting.

\subsection{Disease control summary}

We have examined three epidemiological models. In the first of these we have investigated pulse and constant vaccination strategies in an SIR model for a non-fatal disease in which the total population grows exponentially and the contact rate grows with the population. We were motivated to consider such a model by these observations: human populations have been growing steadily for decades and this trend is predicted to continue; higher contact rates have been linked to higher populations; and within many populations there circulate diseases with low mortality. We discovered that a pulse vaccination strategy has no long-term effect at all, whereas a constant vaccination strategy reduces the long-term proportion of infectives. Although the model itself is valid only on a limited time scale (populations are unlikely to grow forever), we are forced to conclude that pulse vaccination strategies may not always be as effective as the literature has so far suggested.

Our second model was for a disease with SIR-type dynamics for a population living on $n$ patches between any pair of which migration is allowed. We supposed that a pulse vaccination strategy (PVS) is carried out on each patch. We derived conditions on each PVS such that the disease will be eradicated on all patches. The PVS on one patch is essentially independent of the PVS on the other patches except in so far as they are all performed simultaneously. This independence is of practical value when we bear in mind that the patches may represent regions with autonomous public health authorities, which may make individual decisions about the days appropriate for a vaccination pulse to occur in their own region.

Since gestation delays have largely been ignored in the literature of mathematical epidemiology, at least in terms of studying mammalian populations, our final model was for a mammalian disease with SIR-type dynamics in which a gestation delay 
is included. The model was derived under fairly general assumptions. There was no vertical transmission but disease-caused deaths were allowed and the conception function was of a form that could allow either year-round breeding or breeding during a single annual mating season. We explored the behaviour of the model in the special case where breeding took place year-round and the disease was non-fatal, a scenario which could describe a mild disease in a human population. Having proved that the disease can be naturally endemic, we constructed pulse and constant vaccination strategies that eradicate it. We then derived and briefly looked at two models for insect pathogens, noting that one of these models reduced, in certain circumstances, to our mammalian SIR model with a gestation delay. We were led to suggest that existing models in the literature of insect pathogen dynamics be viewed in a wider context for they may yield applications to mammalian populations.

\subsection{Disease control extensions}

There are various ways to extend the disease control research conducted in this thesis. Our SIR model for a non-fatal disease in an exponentially growing population with a growing contact rate could be generalised by the inclusion of disease fatalities or by the introduction of an exposed class. The model could be made more realistic over a longer time scale if, as the population grew above a certain level, the birth rate were allowed to fall or the contact rate were allowed to saturate. Saturating and nonlinear incidences have already been considered in a number of other situations $[78,94,71$, 140].

For our SIR model on $n$ patches, we could generalise our results by seeking the least restrictive conditions on the pulse vaccination strategy on each patch such that the disease will still be eradicated. Again, an exposed class could be built into the model. The open problem of rigorously establishing endemicity in the model without vaccinations could obviously also be explored.

For our mammalian SIR model with a gestation delay, an examination could be carried out when disease-caused deaths are allowed and when mating takes place during a single annual mating season. Such a situation may describe a disease circulating in a herding animal including deer or cattle. Further, an SIR model (or models with other disease dynamics such as SEIR or SEIRS) could be derived when there is a gestation delay and vertical transmission may occur. It may also be fruitful to seek mammalian applications for existing entomopathogen models.

We have alluded a number of times in this thesis to the observation by Choisy et al that infrequent pulses in a pulse vaccination strategy may exacerbate an outbreak of a disease by causing resonance in the underlying dynamical system [61]. We also provided our own evidence, in the form of simulations in two of our epidemiological models, of how infrequent pulses can, over a limited time span, increase the number or proportion of infectives. To our knowledge no analytical demonstration of the potential harm of infrequent pulses has been given. In view of our proof in chapter 4 that infrequent culling in a pest control model can increase the average population of a pest, we suggest, as future research, the adaptation of the techniques of chapter 4 to establish that infrequent vaccination pulses can increase the average infective population in an epidemiological model.

Finally we can envisage an intriguing problem that would involve both pest control and disease control. A model could be formulated for the spread of a vector-borne disease in a human population. The vector is known to have a stage-structured life 
cycle (like a mosquito) and a particular pesticide is known to be quite effective in killing the adults. Also a vaccination exists for the disease in the human population. The combined influence on the disease dynamics of adult impulsive culling of the vector and pulse vaccinating the human population could be investigated. In particular, we could ask, when is the disease eradicated and when is it not? Also, can the two different impulsive regimes combine to lead to a very quick disease eradication or can they interfere with each other and cause the disease to fare better than with only pest control or only disease control or no control of any kind? After all, we have seen in this thesis that infrequent culling can benefit a pest, and it is known from the literature that a pulse vaccination strategy can make a disease infect more people can both phenomena occur at the same time? 


\section{Bibliography}




\section{Bibliography}

[1] http: //ppathw3.cals.cornell.edu/mba_project/moist/home2.html

[2] http://www.health.qld.gov.au/chrisp/images/syringe2.jpg.

[3] 2004 UN population report. http://www.un.org/esa/population/publications/sixbillion/sixbil part1.pdf.

[4] Adult stage of Codling Moth. http://www.btinternet.com/ micka.wffps/codling_moth.jpg.

[5] Article on Colorado potato beetle. http://en.wikipedia.org/wiki/Colorado_potato_beetle.

[6] Article on Mosquitoes. http://en.wikipedia.org/wiki/Mosquita.

[7] Biological control information center. http://cipm.ncsu. edu/ent/biocontrol/.

[8] Biological Notes on Mosquitoes. http: //www. mosquitoes.org/Lifecycle.html.

[9] Breakdown products of widely used pesticides are acutely lethal to amphibians, study finds. Sciencedaily. com.

[10] Breedinghabitat source reduction. http: //dhs.wisconsin.gov/eh/Mosqui tocontrol/HabitatReductio n.htm.

[11] Caterpillar stage of Codling Moth. http: //upload.wikimedia.org/wikipedia/commons/c/c6/Madige-Ap fel-Frucht.jpg.

[12] Cocoon stage of Codling Moth. http: //agspsrv34.agric.wa.gov.au/ento/_fpclass/codling4.jpg.

[13] The Diptera Site: TephritidaePhylogeny. http://www.sel .barc.usda.gov/Diptera/tephriti/TephPhyl . htm.

[14] Economic impacts of the boll weevil. http://bollweevil.ext.msstate.edu/webpage_history.htm

[15] Egg stage of Codling Moth. http: //www. Iivingwithbugs.com/Images/codling_moth_eggs.jpg.

[16] Leptinotarsa decemlineata (TSN: 720110), Integrated Taxonomic Information System. http: //www. itis.gov/servlet/SingleRpt/SingleRpt?search_topic=TSN $\backslash \&$ search_value $=720110$.

[17] The Endopterygote Group. http: //www.cals.ncsu.edu/course/ent 425/text02/holometabola.html.

[18] Global Corruption Barometer 2005. http: //www.transparency.org/news_room/latest_news/press_re leases/2005/09_12_2005_barometer_2005.

[19] Global Polio Eradication Initiative: The history. http: //www.polioeradication.org/history.asp

[20] HIV and AIDS discrimination and stigma (Avert charity web site). http: //www . avert.org/aidsstigma.htm

[21] Integrated pest management. http://cipm.ncsu. edu/.

[22] Killing off the cane toad. http: //www. imb. uq. edu. au/index. html?page $=48437$.

[23] The Mammal Society: Fact Sheet on Red Deer. http: / /www.abdn.ac.uk/mammal/red_deer.shtml

[24] Mosquito-Borne Diseases. http://www.cdc.gov/ncidod/diseases/list_mosquitoborne.htm

[25] Public Health Image Library (PHIL), Centers for Disease Control and Prevention (CDC), image 10707. http://phil.cdc.gov/phil/details.asp. 
[26] UC Pest Management Guidelines, Apple Codling Moth. http: //www . ipm. ucdavis . edu/ PMG/r4300111. htm]

[27] World Health Organisation Fact Sheet on Measles. http://www. who. int/mediacentre/factsheets /fs 286 /en/index.html.

[28] World Health Organization article on smallpox. http: //www. who. int/mediacentre/factsheets/smallpo $\mathrm{x} / \mathrm{en} /$.

[29] World Health Organization: Dracunculiasis eradication initiative. http: //www . who. int/dracunculiasis/e radication/en/.

[30] World Health Organization: Immunization, Vaccines, and Biologicals, (Note: Please choose appropriate disease). http://www. who.int/immunization/topics/en/.

[31] World Health Organization: WHO Vaccine Preventable Diseases Monitoring System. http://www. who.int/vaccines/globalsummary/immunization/scheduleselect.cfm.

[32] The World Health Report 1995 (table 5). http: //www.who.int/whr/1995/en/whr95_ch1_en.pdf.

[33] The World Health Report 2004. http://www. who.int/whr/2004/annex/topic/en/annex_2_en.pdf.

[34] World Population Prospects: The 2006 Revision Population Database (United Nations database). http://esa.un.org/unpp.

[35] Eradication of dracunculiasis, 1997. http://www. who.int/dracunculiasis/eradication/WHA50.35.pdf.

[36] Controversy dogs Aids forum: BBC news article, 2000. http://news . bbc.co. uk/1/hi/world/africa/82674 2.stm.

[37] Report of the High-Level Mission on the situation of human rights in Darfur pursuant to Human Rights Council Decision S-4/101, 2005. http: //news.bbc.co.uk/1/shared/bsp/hi/pdfs/12_03_07_un_sudan.pdf.

[38] UN's Darfur death estimate soars, 2005. http://news .bbc.co.uk/1/hi/world/africa/4349063.stm.

[39] Fall Dwindle Disease: A preliminary report, 2006. http: //maarec .cas . psu. edu/pressReleases/FallDwin dleUpdate0107.pdf.

[40] Cholera is Raging, Despite Denial by Mugabe: New York Times article, 2008. http: //www nytimes.com/2008/12/12/world/africa/12 cholera.html?hp.

[41] MSF responding to worst cholera outbreak in Zimbabwe in years: MSF article, 2008. http: //www.msf.org/ms finternational/invoke.cfm?objectid=3A06C714-15C5-F00A-25B61038A 975AFAB\&component=toolkit . article\&method=full_html.

[42] Zimbabwe "asks for cholera help": BBC news article, 2008. http: //news . bbc.co. uk/1/hi/world/africa/7 $763397 . \mathrm{stm}$.

[43] Physicians: Corruption killing people in Zimbabwe, 2009. http: //www . iht.com/articles/ap/2009/01/13/ africa/AF-Zimbabwe.php.

[44] Z. Agur, L. Cojocaru, G. Mazor, R. M. Anderson, and Y. L. Danon. Pulse mass measles vaccination across age cohorts. Population Biology, 90:11698-11702, 1993.

[45] W. C. Allee. The Social Life of Animals. Heinemann, London, 1931.

[46] R. M. Anderson. Transmission dynamics and control of infectious disease agents, pages 149-176. Springer, Berlin, 1982.

[47] R. M. Anderson and R. M. May. Infectious Diseases of Humans. Oxford University Press, 1991.

[48] J. Arino, J. R. Davis, D. Hartley, R. Jordan, J. M. Miller, and P. van den Driessche. A multi-species epidemic model with spatial dynamics. Mathematical Medicine and Biology, 22:129-142, 2005.

[49] J. Arino and P. van den Driessche. Disease spread in metapopulations. Fields Institute Communications, 48, 2006.

[50] I. Arita, J. Wickett, and F. Fenner. Impact of population density on immunization programmes. Journal of Hygiene, (96):459-466, 1986.

[51] N. T. J. Bailey. The mathematical theory of infectious diseases and its applications. Charles Griffin and Company Ltd, second edition, 1975 .

[52] D. Bernoulli. Essai d'une nouvelle analyse de la mortalité causée par la petite vérole et des advantages de l'inoculation pour le prévenir. Mém. Math. Phys. Acad, Roy. Sci., Paris, pages 1-45, 1760. 
[53] J. M. Bonmatin, P. A. Marchand, R. Charvet, I. Moineau, E. R. Bengsch, and M. E. Colin. Quantification of imidacloprid uptake in maize crops. J. Agric. Food Chem., 53(13):5336-5341, 2005.

[54] F. Brauer. A model for an SI disease in an age-structured population. Discrete and Continuous Dynamical Systems, (2):257-264, 2002.

[55] F. Brauer and C. Castillo-Chávez. Mathematical Models in Population Biology and Epidemiology. Springer-Verlag, New York, 2001

[56] C. J. Briggs and H. C. J. Godfray. The dynamics of insect-pathogen dynamics in stage-structured populations. Am. Nat., (145):855-887, 1995.

[57] N. Broderick, K. Raffa, and J. Handelsman. Midgut bacteria required for Bacillus thuringiensis insecticidal activity. Proceedings of the National Academy of Sciences of the United States of America, 103(41):1519615199, 2006.

[58] G. C. Brown. Stability in an insect-pathogen model incorporating age-dependent immunity and seasonal host reproduction. Bulletin of Mathematical Biology, 46(1):139-153, 1984

[59] M. Burton, editor. Encyclopaedia of Animals. Cathay Books, 1987.

[60] S. N. Busenberg and P. van den Driessche. Analysis of a disease transmission model in a population with varying total size. Journal of Mathematical Biology, (28):257-270, 1990.

[61] M. Choisy, J-F. Guegán, and P. Rohani. Dynamics of infectious diseases and pulse vaccination: Teasing apart the embedded resonance effects. Physica D, (223):26-35, 2006.

[62] C. J. Clements, P. Greenough, and D. Schull. How vaccine safety can become political the example of polio in Nigeria. Current Drug Safety, 1(1):117-119, 2006. http://bentham. org/cds/samples/cds1-1/Clements . pdf.

[63] J. D. Cornell. Slash and burn. Encyclopedia of Earth. http://www. eoearth.org/article/Slash_and_burn

[64] H. Daly, J. T. Doyen, and A. H. Purcell III. Introduction to insect biology and diversity. Oxford University Press, New York, second edition, 1998.

[65] M. C. M. de Jong, O. Diekmann, and H. Heesterbeek. How does transmission of infection depend on population size?, pages 84-94. Publications of the Newton Institute, vol. 5, Cambridge University, Cambridge, 1995.

[66] V. Demicheli, T. Jefferson, A. Rivetti, and D. Price. Vaccines for measles, mumps and rubella in children. Cochrane Database Syst. Rev., 19(4), 2005.

[67] O. Diekmann, M. C. M. de Jong, and J. A. J. Metz. A deterministic epidemic model taking account of repeated contacts between the same individuals. Journal of Applied Probability, (35):448-462, 1998.

[68] A. F. G. Dixon. Aphid Ecology: An Optimization Approach. Chapman and Hall, 1998.

[69] A. d'Onofrio. Pulse vaccination strategy in SIR epidemic model: Global asymptotic stable eradication in presence of vaccine failures. Mathematical Computer Modelling, 36(4-5):473-489, 2002.

[70] A. d'Onofrio. Stability properties of pulse vaccination stategy in SEIR epidemic model. Mathematical Biosciences, (179):57-72, 2002.

[71] A. d'Onofrio. Vaccination policies and nonlinear force of infection: generalisation of an observation by Alexander and Moghadas (2004). Applied Mathematics and Computation, (168):613-622, 2005.

[72] B. Dybiec, A. Kleczkowski, and C. A. Gilligan. Controlling disease spread on networks with incomplete knowledge. Phys. Rev. E, 70(066145), 2004.

[73] D. J. D. Earn, P. Rohani, and B. T. Grenfell. Persistence, chaos, and synchronicity in ecology and epidemiology. Proc. R. Soc. Lond. B, (265):7-10, 1998.

[74] R. F. Edlich, K. L. Winters, W. B. Long, and K. D. Gubler. Rubella and congenital rubella (German measles). J Long Term Eff Med Implants, 15(3):319-328, 2005.

[75] C. H. Edwards and D. E. Penney. Elementary Differential Equations With Boundary Value Problems. Prentice-Hall, New Jersey, third edition, 1993.

[76] M. Fan, M. Y. Li, and K. Wang. Global stability of an SEIS epidemic model with recruitment and a varying total population size. Mathematical biosciences, (170):199-208, 2001.

[77] J. R. Fuxa and Y. Tanada, editors. Epizootiology of Insect Diseases. Wiley-Interscience, 1987.

[78] S. Gao, L. Chen, J. J. Nieto, and A. Torres. Analysis of a delayed epidemic model with pulse vaccination and saturation incidence. Vaccine, (24):6037-6045, 2006. 
[79] K. Gopalsamy. Stability and Oscillations in Delay Differential Equations of Population Dynamics. Kluwer Academic Publishers, 1992.

[80] K. Gopalsamy and B. G. Zhang. On delay differential equations with impulses. (139):110-122, 1989.

[81] S. A. Gourley, R. Liu, and J. Wu. Eradicating vector-born diseases via age-structured culling. Journal of Mathematical Biology, 54(3):309-335, 2007.

[82] W. Gurney, S. Blythe, and R. Nisbet. Nicholson's blowflies revisited. Nature, (287):17-21, 1980.

[83] W. Gurney, R. Nisbet, and J. Lawton. The systematic formulation of tractable single-species population models incorporating age structure. The Journal of Animal Ecology, 52(2):479-495, 1983.

[84] W. H. Hamer. Epidemic disease in england. The Lancet, (1):733-739, 1906.

[85] World Health Organization Press Release (2/11/2000). Pulse Polio Campaign Targets over 107 Million Children in 11 States. http://WWw. searo. who. int/EN/Section316/Section503/Section2373_12973.htm

[86] M. Heino, V. Kaitala, E. Ranta, and J. Lindstrom. Synchronous dynamics and rates of extinction in spatially structured populations. Proc. R. Soc. Lond. B, (264):481-486, 1997.

[87] H. W. Hethcote. The Mathematics of Infectious Diseases. SIAM review, 42(4):599-653, 2000.

[88] Y. Jin and W. Wang. The effect of population dispersal on the spread of a disease. J. Math. Anal. Appl., 308:343-364, 2005. [89] D. L. Karlen, G. E. Varvel, D. G. Bullock, and R. M. Cruse. Crop rotations for the 21st century. Advances in Agronomy,
53:1-45, 1994.

[90] M. J. Keeling. Correlation equations for endemic diseases: externally imposed and internally generated heterogeneity. Proc. R. Soc. Lond., (266):953-960, 1999.

[91] M. J. Keeling. The effects of local spatial structure on epidemiological invasions. Proc. R. Soc. Lond., (266):859-867, 1999.

[92] R. L. Kellogg, R. Nehring, A. Grube, D. W. Goss, and S. Plotkin. Environmental indicators of pesticide leaching and runoff from farm fields, February 2000. Web article.

[93] W. O. Kermack and A. G. McKendrick. A contribution to the mathematical theory of epidemics. Proc. R. Soc., A115:700-721, 1927.

[94] A. Korobeinikov and P. K. Maini. Non-linear incidence and stability of infectious disease models. Mathematical Medicine and Biology, 22:113-128, 2005.

[95] Y. Kuang. Delay Differential Equations with Applications in Population Dynamics. Academic Press, Inc., 1993.

[96] S. Kuniuki. Effects of organic fertilization and pesticide application on growth and yield of field-grown rice for 10 years. Japanese Journal of Crop Science, 70(4):530-540, 2001.

[97] L. A. Lacey, R. Frutos, H. K. Kaya, and P. Vail. Insect pathogens as biological control agents: do they have a future? Biological Control, (21):230-248, 2001.

[98] M. Y. Li, J. R. Graef, L. Wang, and J. Karsai. Global dynamics of a SEIR epidemic model with varying total population size. Mathematical biosciences, (160):191-213, 1999.

[99] M. Y. Li, H. L. Smith, and L. Wang. Global dynamics of an SEIR epidemic model with vertical transmission. SIAM journal of Applied Mathematics, 62.

[100] F. Liljeros, C. R. Edling, L. A. N. Amaral, H. E. Stanley, and Y. Aberg. Human web of sexual contacts. Nature, (411):907-908, 2001.

[101] A. L. Lloyd and V. A. A. Jansen. Spatiotemporal dynamics of epidemics: synchrony in metapopulation models. Mathematical Biosciences, (188):1-16, 2004.

[102] H. McCallum, N. Barlow, and J. Hone. How should pathogen transmission be modelled? Trends in Ecology and Evolution, 16(6):295-300, 2001.

[103] P. McIntyre and J. Leask. Improving uptake of MMR vaccine. BMJ, 336(7647):729-730, 2008.

[104] W. H. McNeill. Plagues and Peoples. Doubleday, New York, 1976.

[105] W. H. McNeill. The Global Condition. Princeton University Press, Princeton, New Jersey, 1990. 
[106] J. Mena-Lorca and H. W. Hethcote. Dynamic models of infectious diseases as regulators of population sizes. Journal of Mathematical Biology, 30(7):693-716, 1992.

[107] J. A. J. Metz and O. Diekmann, editors. The Dynamics of Physiologically Structured Populations. Springer-Verlag, New York, 1986.

[108] G. T. Miller. Living in the Environment. Belmont: Wadsworth/Thomson Learning, twelfth edition, 2002.

[109] G. T. Miller. Sustaining the Earth. Thompson Learning, Inc. Pacific Grove, California, sixth edition, 2004.

[110] K. Mischaikow, H. Smith, and H. R. Thieme. Asymptotically Autonomous Semiflows: Chain Recurrence and Lyapunov Functions. Transactions of the American Mathematical Society, 345(5):1669-1685, 1995.

[111] M. Moerbeek and F. van den Bosch. Insect-pathogen dynamics: stage-specific susceptibility and insect density dependence. Mathematical Biosciences, (141):115-148, 1997.

[112] J. D. Murray. Mathematical Biology. Springer-Verlag, second edition, 1993.

[113] M. E. J. Newman. Spread of epidemic disease on networks. Phys. Rev. E, 66(016128), 2002.

[114] BBC news article. South Africa to allow elephant cull. http://news . bbc.co.uk/1/hi /world/africa/72629 $51 . \mathrm{stm}$.

[115] A. J. Nicholson. An outline of the dynamics of animal populations. Australian Journal of Zoology, (2):9-65, 1954.

[116] A. J. Nicholson. The self-adjustment of populations to change. Cold Spring Harbour Symposium on Quantitative Biology, (22):153-173, 1957.

[117] C. O'Toole, editor. The encyclopedia of insects. Equinox (Oxford) Ltd., 1987.

[118] J. M. Read and M. J. Keeling. Disease evolution on networks: the role of contact structure. Proc. R. Soc. Lond., (270):699-708, 2003.

[119] M. B. Renfree and B. Shaw. Diapause. Annual Review of Physiology, 62:353-375, 2000.

[120] C. Roddeck and M. Whittle. Fetal Medicine: Basic Science and Clinical Practice. Elsevier Health Sciences, 1999.

[121] R. Ross. The prevention of malaria. Murray, London, second edition, 1911.

[122] E. V. Shakhov and V. N. Krupin. The clinico-statistical characteristics of the testicular generative function in male subfertility following mumps. Urol Nefrol (Mosk), 2:46-50, 1990.

[123] B. Shulgin, L. Stone, and Z. Agur. Pulse vaccination stategy in the SIR epidemic model. Bulletin of Mathematical Biology, (60):1123-1148, 1998.

[124] M. Siegel, H. T. Fuerst, and V. F. Guinee. Rubella epidemicity and embryopathy. Results of a long-term prospective study. Am. J. Dis. Child., 121(6):469-473, 1971.

[125] R. R. L. Simons and S. A. Gourley. Extinction criteria in stage-structured population models with impulsive culling. SLAM Journal of Applied Mathematics, 66(6):1853-1870, 2006

[126] M. Small, P. Shi, and C. K. Tse. Plausible models for propagation of the sars virus. IEIEC Trans. Fundamentals, E87-A(9):2379-2386, 2004. http: //arxiv.org/PS_cache/q-bio/pdf/0312/0312029v1 . pdf.

[127] H. L. Smith. A structured population model and a related functional-differential equation: global attractors and uniform persistence. Journal of Dyn. Diff. Equ., (6):71-99, 1994.

[128] H. L. Smith. Monotone Dynamical Systems: An Introduction to the Theory of Competitive and Cooperative Systems. American Mathematical Society, Providence, Rhode Island, 1995.

[129] J. W.-H. So, J. Wu, and X. Zou. Structured population on two patches: modelling dispersal and delay. Mathematical Biology, (43):37-51, 2001.

[130] D. Stoffer. Delay equations with rapidly oscillating stable periodic solutions. Journal of Dynamics and Differential Equations, 20(1):201-238, 2008.

[131] S. H. Strogatz. Nonlinear Dynamics and Chaos. Perseus Books Publishing, LLC, 1994.

[132] Editorial team. Measles once again endemic in the United Kingdom. Eurosurveillance, 13(27):18919, 2008. http: //www. eurosurveillance.org/ViewArticle. aspx?ArticleId=18919.

[133] A. J. Terry. Impulsive adult culling of a tropical pest with a stage-structured life cycle. Nonlinear Dynamics: Real World Applications, doi: 10.1016/j.nonrwa.2009.01.005, 2009. http://dx.doi.org/10.1016/j.nonrwa.2009.01.005. 
[134] P. Tobin, C. Robinet, D. Johnson, S. Whitmire, O. Bjornstad, and A. Liebhold1. The role of allee effects in gypsy moth, Lymantria dispar (1.), invasions. Population Ecology, 51(3):373-384, 2009.

[135] V. M. Trenkel. Exploring red deer culling strategies using a population-specific calibrated management model. Journal of Environmental Management, 62(1):37-53, 2001.

[136] R. K. Upadhyay, editor. Advances in Microbial Control of Insect Pests. Springer, 2003.

[137] A. J. Wakefield, S. H. Murch, A. Anthony, J. Linnell, D. M. Casson, M. Malik, M. Berelowitz, A. P. Dhillon, M. A. Thomson, P. Harvey, A. Valentine, S. E. Davies, and J. A. Walker-Smith. Ileal-lymphoid-nodular hyperplasia, nonspecific colitis, and pervasive developmental disorder in children. The Lancet, 351(9103):637-641, 1998.

[138] Y. Xiao and F. van den Bosch. The dynamics of an eco-epidemic model with biological control. Ecological Modelling, 168(1-2):203-214, 2003.

[139] T. Yamanaka and A. Liebhold. Spatially implicit approaches to understand the manipulation of mating success for insect invasion management. Population Ecology, 51:427-444, 2009.

[140] J. Zhang and Z. Ma. Global dynamics of an SEIR epidemic model with saturating contact rate. Mathematical Biosciences, 185(1):15-32, 2003. 


\section{Index}




\section{Index}

Allee effect, 13

on a single patch, 28

on two patches, 65

biological pest control, 10

birth function

Allee, 13

linear, 12

Nicholson, 13

step, 45

boll weevil, 8

cholera

in Zimbabwe, 123

codling moth, 6

cohort, 169

Colony Collapse Disorder, 36

colorado potato beetle, 5

contact rate, 119,127

corruption, 123

crop rotation, 10

culling

infrequent, 41

of elephants in South Africa, 197

of red deer in Scotland, 197

stage-specific, 7

Darfur

civil conflict, 122

DDT, 8

dracunculiasis

eradication goal, 146

endemicity

of a disease, 118

of a pest, 15

Endopterygota, 5

entomopathogen, 184

epidemic, 118

eradication of a disease absolute, 121

relative, 121

exposed

as a disease status, 119

fruit fly, 6

gestation

delayed implantation, 167

mammalian examples, 168

GM crops, 10

HIV/AIDS

in South Africa, 123

mortality, 120

stigma, 123

homogeneous mixing, 119

honeybee, 8,36

incidence, 119

function, 127

intermediate mass action, 127

nonlinear, 198

saturating, 198

simple mass action, 127

standard, 127

infective

as a disease status, 118

insect life cycles, 5

Integrated Pest Management (IPM), 10

malaria

mortality, 120

mass action principle, 119

mathematical epidemiology

history, 117

types of model, 118

maturation age, 12, 29

measles

in Central and South America, 121 
in the UK, 123

mortality, 120

virion, 124

method of steps, 15

miscarriage, 169

MMR (Measles, Mumps, Rubella vaccine) controversial link to autism, 123

mortality data for infectious diseases, 120

mosquito, 1, 29

mumps

as a cause of male infertility, 166

patch, 7

pertussis (whooping cough) mortality, 120

pesticides advantages and disadvantages, 8

poliomyelitis

eradication goal, 146

in Central and South America, 122

in India, 122

in Nigeria, 124

population

density, 125

metapopulation, 147

world historical and predicted, 126

positivity, 12

removed

as a disease status, 118

rubella

congenital rubella syndrome, 166

SARS, 165

slash and burn agriculture, 10

slash/mulch, 10

smallpox

eradication, 121

historical influence, 121

incidence, 125

technique of variolation, 117

stage-structured life cycle, 5

susceptible

as a disease status, 118

vaccination strategy

constant, 121

mixed, 121 pulse, 121

real-world successes, 121

varicella (chickenpox), 125

vector-borne diseases, 1

vertical transmission, 119

World Health Organisation, 8, 146 